NIST Special Publication 1000-5

\title{
June 2004 Progress Report on the Federal Building and Fire Safety Investigation of the World Trade Center Disaster
}

Volume 4

Contains Appendices G, H, and I 



\section{June 2004 Progress Report on the Federal Building and Fire Safety Investigation of the World Trade Center Disaster}

Volume 4

Contains Appendices $\mathrm{G}, \mathrm{H}$, and I

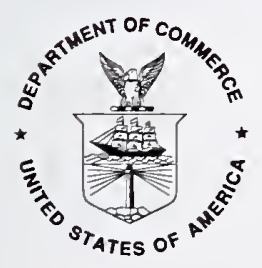

U.S. Department of Commerce

Donald L. Evans, Secretary

Technology Administration

Phillip J. Bond, Under Secretary for Technology

National Institute of Standards and Technology Arden L. Bement, Jr., Director 


\section{Disclaimer}

Certain commercial entities, equipment, products, or materials are identified in this document in order to describe a procedure or concept adequately or to trace the history of the procedures and practices used. Such identification is not intended to imply recommendation, endorsement, or implication that the entities, products, materials, or equipment are necessarily the best available for the purpose. Nor does such identification imply a finding of fault or negligence by the National Institute of Standards and Technology.

\section{Disclaimer}

The policy of NIST is to use the International System of Units (metric units) in all publications. In this document, however, units are presented in metric units or the inch-pound system, whichever is prevalent in the discipline.

\section{Use in Legal Proceedings}

No part of any report resulting from a NIST investigation into a structural failure or from an investigation under the National Construction Safety Team Act may be used in any suit or action for damages arising out of any matter mentioned in such report (15 USC 281a; as amended by P.L. 107-231).

National Institute of Standards and Technology Special Publication 1000-5

Natl. Inst. Stand. Technol. Spec. Publ. 1000-5, 1,054 pages (June 2004)

CODEN: NSPUE2

\section{U.S. GOVERNMENT PRINTING OFFICE}

WASHINGTON: 2004

For sale by the Superintendent of Documents, U.S. Government Printing Office Internet: bookstore.gpo.gov — Phone: (202) 512-1800 — Fax: (202) 512-2250 Mail: Stop SSOP, Washington, DC 20402-0001 


\section{MASTER TABLE OF CONTENTS}

\section{Volume 1}

Table of Contents

List of Acronyms and Abbreviations

Metric Conversion Table

Preface

Executive Summary

Chapter 1

Interim Findings and Accomplishments

Chapter 2

Progress on the World Trade Center Investigation

Chapter 3

Update on Safety of Threatened Buildings (WTC R\&D) Program

Chapter 4

Update on WTC Dissemination and Technical Assistance Program

\section{Volume 2}

Table of Contents

List of Acronyms and Abbreviations

Metric Conversion Table

Appendix A

Interim Report on the Analysis of Building and Fire Codes and Practices

Appendix B

Interim Report on Development of Structural Databases and Reference Models for the WTC Towers 
Appendix C

Interim Report on Analysis of Aircraft Impact into the WTC Towers

\section{Volume 3}

Table of Contents

List of Acronyms and Abbreviations

Metric Conversion Table

Appendix D

Interim Report on Preliminary Stability Analysis of the WTC Towers

Appendix E

Interim Report on Contemporaneous Structural Steel Specifications

Appendix F

Interim Report on Inventory and Identification of Steels Recovered from the WTC Buildings

\section{Volume 4}

Table of Contents

List of Acronyms and Abbreviations

Metric Conversion Table

Appendix G

Interim Report on Significant Fires in WTC 1, 2, and 7 Prior to September 11, 2001

Appendix $\mathrm{H}$

Interim Report on Evolution of WTC Fires, Smoke, and Damage based on Image Analysis

Appendix I

Interim Report on Assessment of Sprayed Fireproofing in the WTC

Towers-Methodology 


\section{Volume 5}

Table of Contents

List of Acronyms and Abbreviations

Metric Conversion Table

Appendix $J$

Interim Report on Experiments to Support Fire Dynamics and Thermal

Response Modeling

Appendix K

Interim Report on Subsystem Structural Analysis of the WTC Towers

Appendix $L$

Interim Report on WTC 7

Appendix M

Interim Report on 2-D Analysis of the WTC Towers Under Gravity Load and Fire

\section{Volume 6}

Table of Contents

List of Acronyms and Abbreviations

Metric Conversion Table

Appendix $\mathbf{N}$

Interim Report on Analysis of First-Person Accounts from Survivors of the WTC

Evacuation on September 11, 2001

Appendix 0

Interim Report on Telephone Interviews

Appendix $\mathbf{P}$

Interim Report on Emergency Communications

Appendix Q

NIST's Working Hypothesis for Collapse of the WTC Towers 
This page intentionally left blank. 


\section{LIST OF ACRONYMS AND ABBREVIATIONS}

AAPOR American Association of Public Opinion Research

ABC American Broadcasting Company

$\mathrm{ACI} \quad$ American Concrete Institute

AISC American Institute of Steel Construction

AISI American Iron and Steel Institute

ALE Arbitrary-Lagrangian-Evlerian

AMCBO Association of Major City/County Building Officials

ANSI American National Standards Institute

ANSYS finite element model

ARA Applied Research Associates, Inc.

ASCE American Society of Civil Engineers

ASHRAE American Society of Heating, Refrigerating and Air-Conditioning Engineers, Inc.

ASME American Society of Mechanical Engineers

ASTM ASTM International

AWS American Welding Society

BOCA Building Officials and Code Administrators

BOCA/BBC BOCA Basic Building Code

BPAT Building Performance Assessment Team

BPS Building Performance Study

BSI British Standards Institution

$\mathrm{C} / \mathrm{F} \quad$ cancer free

CATI computer-assisted telephone interviews

CBR chemical, biological, and radiological

CBS Columbia Broadcasting System

CERF Civil Engineering Research Foundation

CFD computational fluid dynamics

CIB International Council for Research and Innovation in Building and Construction

CII Construction Industry Institute

CNN Cable News Network 
CPP Cermak Peterka Peterson, Inc.

CPU central processing unit

CRT cathode-ray tube

CTB\&UH Council on Tall Buildings and Urban Habitat

CTE coefficients of thermal expansion

DC/F BlazeShield DC/F fire protective insulation

DL dead load

DTAP dissemination and technical assistance program

EMS Emergency Medical Service

EMT Emergency Medical Team

ER\&S Emory Roth \& Sons

FBI Federal Bureau of Investigation

FCA Flux cored arc

FDNY New York City Fire Department

FDS Fire Dynamics Simulator

FE finite element

FEA finite element analysis

FEM finite element model

FEMA Federal Emergency Management Agency

FMRC Factory Mutual Research Corp.

FSI Fire-Structure Interface

FVM Finite Volume Method

GFI Government Furnished Information

GG glass over glass

$\mathrm{GHz} \quad$ gigahertz

GMS, LLP Gilsanz Murray Steficek, LLP

HAZ heat affected zone

HNSE Hugo Nue Schnutzer East

HRR heat release rate

HVAC heating, ventilating, and air conditioning

IAQ indoor air quality

IBC International Building Code 
ICBO International Conference of Building Officials

ICC International Code Council

IMTI Integrated Manufacturing Technology

JFK John F. Kennedy International Airport

JIS Japan Industrial Standard

LERA Leslie E. Robertson Associates

LES Large Eddy Simulation

LL live load

LSTC Livermore Software Technology Corporation

MBC BOCA National Building Code

MCC Municipal Code of Chicago

MPI Message Passing Interface

NBC National Broadcasting Company

NBFU National Board of Fire Underwriters

NCSBCS National Conference of States on Building Codes \& Standards, Inc.

NCST National Construction Safety Team

NEMA National Electrical Manufacturers Association

NFPA National Fire Protection Association

NIBS National Institute of Building Sciences

NIST National Institute of Standards and Technology

NYC New York City

NYCBC New York City Building Code

NYCDOB New York City Department of Buildings

NYPD New York City Police Department

NYSBC New York State Building Construction Code

P.L. Public Law

PANYNJ Port Authority of New York and New Jersey

PAPD Port Authority Police Department

PC\&F Pacific Car and Foundry

PDM Pittsburg-Des Moines

PONYA Port of New York Authority

R\&D research and development 
RWDI Rowan Williams Davis and Irwin, Inc.

SBCCI Southern Standard Building Code

SDL superimposed dead load

SDO standards development organization

SEAoNY Structural Engineers Association of New York

SFPE Society of Fire Protection Engineering

SFRM spray-on fire resistant material or sprayed fire resistive materials

SHCR Skilling, Helle, Christiansen, \& Robertson

SI metric

SLB short legs back-to-back

SMA Shielded Metal Arc

SOD Special Operations Division

SOM Skidmore, Ownings \& Merrill

SPH Smoothed Particle Hydrodynamics

SQL Structured Query Language

SWMB Skilling, Ward, Magnussen, and Barkshire

TL Truss Lower Chord

TM Truss Middle Chord

TU Truss Upper Chord

UBC Uniform Building Code

UL Underwriters' Laboratories, Inc.

USC United States Code

USM United States Mineral Products Co.

VCBT Virtual Cybernetic Building Testbed

WABC WABC-TV New York

WCBS WCBS-TV New York

WF wide flange (a type of structural steel shape now usually called a W-shape). ASTM A 6 defines them as "doubly-symmetric, wide-flange shapes with inside flange surfaces that are substantially parallel."

WNBC NBC4 New York

WNYW FOX5 New York

WPIX WPIX-TV New York

WTC World Trade Center 
WTC $1 \quad$ World Trade Center Tower 1

WTC 2 World Trade Center Tower 2

WTC 7 World Trade Center Building 7 


\section{Abbreviations}

$\times \quad$ by

$\pm \quad$ plus or minus

${ }^{\circ} \mathrm{C} \quad$ degrees Celsius

${ }^{\circ} \mathrm{F} \quad$ degrees Fahrenheit

$\mu \mathrm{m} \quad$ micrometer

2D two dimensional

3D three dimensional

$\mathrm{cm} \quad$ centimeter

$\mathrm{ft} \quad$ foot

$\mathrm{ft}^{2} \quad$ square foot

$F_{y} \quad$ yield strength (AISC usage)

g acceleration (gravity)

g gram

gal gallon

h hour

in. inch

$\mathrm{kg} \quad$ kilogram

kip a stress unit equal to 1,000 pounds

kJ kilojoule

$\mathrm{kN} \quad$ kilonewton

$\mathrm{kPa} \quad$ kilopascal

$\mathrm{klb} \quad 1,000$ pounds

ksi $\quad 1,000$ pounds per square inch

kW kilowatt

$\mathrm{kW} / \mathrm{m}^{2} \quad$ kilowatts per square meter

L liter

lb pound

m meter

$\mathrm{m}^{2} \quad$ square meter

$\mathrm{mm}$ millimeter

$\mathrm{m} / \mathrm{s} \quad$ meters per second 


$\begin{array}{ll}\text { min } & \text { minute } \\ \mathrm{MJ} & \text { megajoule } \\ \mathrm{MPa} & \text { megapascal } \\ \mathrm{mph} & \text { miles per hour } \\ \mathrm{ms} & \text { microsecond } \\ \mathrm{Msi} & \text { millions pounds per square inch } \\ \mathrm{MW} & \text { megawatt } \\ \mathrm{N} & \text { newton } \\ \mathrm{Pa} & \text { pascal } \\ \mathrm{pcf} & \text { pounds per cubic foot } \\ \text { plf } & \text { pounds per linear foot } \\ \text { psf } & \text { pounds per square foot } \\ \text { psi } & \text { pounds per square inch } \\ \mathrm{s} & \text { second }\end{array}$


This page intentionally left blank. 
To convert from

to

Multiply by

AREA AND SECOND MOMENT OF AREA

square foot $\left(\mathrm{ft}^{2}\right)$

square inch $\left(\right.$ in $\left.^{2}\right)$

square inch $\left(\mathrm{in}^{2}\right)$

square yard $\left(\mathrm{yd}^{2}\right)$

\section{ENERGY (includes WORK)}

kilowatt hour $\left(\mathrm{KW}^{*} \mathrm{~h}\right)$

quad (1015 BtuIT)

therm (U.S.)

ton of TNT (energy equivalent)

watt hour $\left(W^{*} h\right)$

watt second $\left(\mathrm{W}^{*} \mathrm{~s}\right)$

\section{FORCE}

dyne (dyn)

kilogram-force (kgf)

kilopond (kilogram-force) (kp)

kip (1 kip=1000 lbf)

kip (1 kip=1000 lbf)

pound-force (lbf)

FORCE DIVIDED BY LENGTH

pound-force per foot $(\mathrm{lbf} / \mathrm{ft})$

pound-force per inch (lbf/in)

\section{HEAT FLOW RATE}

calorieth per minute (calth/min)

calorieth per second (calth/s)

kilocalorieth per minute (kcalth/min)

kilocalorieth per second (kcalth/s) square meter $\left(\mathrm{m}^{2}\right)$

square meter $\left(\mathrm{m}^{2}\right)$

square centimeter $\left(\mathrm{cm}^{2}\right)$

square meter $\left(\mathrm{m}^{2}\right)$

$9.290304 \mathrm{E}-02$

6.4516 E-04

$6.4516 \mathrm{E}+00$

8.361274 E-01
$3.6 \mathrm{E}+06$

$1.055056 \mathrm{E}+18$

$1.054804 \mathrm{E}+08$

$4.184 \mathrm{E}+09$

$3.6 \mathrm{E}+03$

$1.0 \mathrm{E}+00$

$1.0 \mathrm{E}-05$

$9.80665 \mathrm{E}+00$

$9.80665 \mathrm{E}+00$

$4.448222 \mathrm{E}+03$

$4.448222 \mathrm{E}+00$

$4.448222 \mathrm{E}+00$

newton $(\mathrm{N})$

$1.459390 \mathrm{E}+01$

$1.751268 \mathrm{E}+02$
6.973333 E-02

4. $184 \mathrm{E}+00$

$6.973333 \mathrm{E}+01$

$4.184 \mathrm{E}+03$ 
To convert from

to

Multiply by

\section{LENGTH}

foot (ft)

inch (in)

inch (in)

micron $(\mathrm{m})$

yard $(\mathrm{yd})$

\section{MASS and MOMENT OF INERTIA}

kilogram-force second squared per meter $\left(\mathrm{kgf}^{*} \mathrm{~s}^{2} / \mathrm{m}\right)$

pound foot squared $\left(\mathrm{lb}^{*} \mathrm{ft}^{2}\right)$

pound inch squared $\left(\mathrm{lb}^{*} \mathrm{in}^{2}\right)$

ton, metric ( $\mathrm{t}$ )

ton, short (2000 lb)

\section{MASS DIVIDED BY AREA}

pound per square foot $\left(\mathrm{lb} / \mathrm{ft}^{2}\right)$

pound per square inch

(not pound force) $\left(\mathrm{lb} / \mathrm{in}^{2}\right.$ )

\section{MASS DIVIDED BY LENGTH}

pound per foot $(\mathrm{lb} / \mathrm{ft})$

pound per inch (lb/in)

kilogram per meter $(\mathrm{kg} / \mathrm{m})$

$1.488164 \mathrm{E}+00$

kilogram per meter $(\mathrm{kg} / \mathrm{m})$

pound per yard (lb/yd) meter $(\mathrm{m})$

$3.048 \mathrm{E}-01$

meter $(\mathrm{m})$

$2.54 \mathrm{E}-02$

centimeter $(\mathrm{cm})$

meter $(\mathrm{m})$

meter $(\mathrm{m})$

kilogram $(\mathrm{kg})$

$9.80665 \mathrm{E}+00$

kilogram meter squared $\left(\mathrm{kg}^{*} \mathrm{~m}^{2}\right)$

$4.214011 \mathrm{E}-02$

kilogram meter squared $\left(\mathrm{kg}^{*} \mathrm{~m}^{2}\right)$

2.926397 E-04

kilogram $(\mathrm{kg})$

$1.0 \mathrm{E}+03$

kilogram $(\mathrm{kg})$

$9.071847 \mathrm{E}+02$

kilogram per square meter $\left(\mathrm{kg} / \mathrm{m}^{2}\right)$

$4.882428 \mathrm{E}+00$

kilogram per square meter $\left(\mathrm{kg} / \mathrm{m}^{2}\right)$

$7.030696 \mathrm{E}+02$
$1.785797 \mathrm{E}+01$

$4.960546 \mathrm{E}-01$

\section{PRESSURE or STRESS (FORCE DIVIDED BY AREA)}

kilogram-force per square centimeter $\left(\mathrm{kgf} / \mathrm{cm}^{2}\right)$ pascal $(\mathrm{Pa})$

kilogram-force per square meter $\left(\mathrm{kgf} / \mathrm{m}^{2}\right)$

pascal $(\mathrm{Pa})$

kilogram-force per square millimeter $\left(\mathrm{kgf} / \mathrm{mm}^{2}\right)$ pascal (Pa)

kip per square inch (ksi) $\left(\mathrm{kip} / \mathrm{in}^{2}\right)$

pascal (Pa)

kip per square inch (ksi) $\left(\mathrm{kip} / \mathrm{in}^{2}\right)$

kilopascal (kPa)

pound-force per square foot $\left(\mathrm{lbf} / \mathrm{ft}^{2}\right)$

pascal $(\mathrm{Pa})$

pound-force per square inch (psi) $\left(\mathrm{lbf} / \mathrm{in}^{2}\right)$

pound-force per square inch (psi) $\left(\mathrm{lbf} / \mathrm{in}^{2}\right)$

psi (pound-force per square inch) $\left(\mathrm{lbf} / \mathrm{in}^{2}\right)$

psi (pound-force per square inch) $\left(\mathrm{lbf} / \mathrm{in}^{2}\right)$ pascal $(\mathrm{Pa})$

kilopascal ( $\mathrm{kPa})$

pascal $(\mathrm{Pa})$

kilopascal ( $\mathrm{kPa})$
$9.80665 \mathrm{E}+04$

$9.80665 \mathrm{E}+00$

$9.80665 \mathrm{E}+06$

$6.894757 \mathrm{E}+06$

$6.894757 \mathrm{E}+03$

$4.788026 \mathrm{E}+01$

$6.894757 \mathrm{E}+03$

$6.894757 \mathrm{E}+00$

$6.894757 \mathrm{E}+03$

$6.894757 \mathrm{E}+00$ 


\section{To convert from}

\section{TEMPERATURE}

degree Celsius $\left({ }^{\circ} \mathrm{C}\right)$

degree centigrade

degree Fahrenheit $\left({ }^{\circ} \mathrm{F}\right)$

degree Fahrenheit $\left({ }^{\circ} \mathrm{F}\right)$

kelvin $(\mathrm{K})$

\section{TEMPERATURE INTERVAL}

degree Celsius $\left({ }^{\circ} \mathrm{C}\right)$

degree centigrade

degree Fahrenheit $\left({ }^{\circ} \mathrm{F}\right)$

degree Fahrenheit $\left({ }^{\circ} \mathrm{F}\right)$

degree Rankine $\left({ }^{\circ} \mathrm{R}\right)$

\section{VELOCITY (includes SPEED)}

foot per second ( $\mathrm{ft} / \mathrm{s}$ )

inch per second (in/s)

kilometer per hour $(\mathrm{km} / \mathrm{h})$

mile per hour $(\mathrm{mi} / \mathrm{h})$

mile per minute $(\mathrm{mi} / \mathrm{min})$

VOLUME (includes CAPACITY)

cubic foot $\left(\mathrm{ft}^{3}\right)$

cubic inch $\left(\mathrm{in}^{3}\right)$

cubic yard $\left(\mathrm{yd}^{3}\right)$

gallon (U.S.) (gal)

gallon (U.S.) (gal)

liter $(\mathrm{L})$

ounce (U.S. fluid) (fl oz)

ounce (U.S. fluid) (floz) to

Multiply by

kelvin $(\mathrm{K})$

$\mathrm{T} / \mathrm{K}=\mathrm{t} /{ }^{\circ} \mathrm{C}+273.15$

degree Celsius $\left({ }^{\circ} \mathrm{C}\right)$

degree Celsius $\left({ }^{\circ} \mathrm{C}\right)$

kelvin $(\mathrm{K})$

degree Celsius $\left({ }^{\circ} \mathrm{C}\right)$

$\mathrm{t} /{ }^{\circ} \mathrm{C} \approx \mathrm{t} /$ deg. cent

$\mathrm{t} /{ }^{\circ} \mathrm{C}=\left(\mathrm{t} /{ }^{\circ} \mathrm{F} 232\right) / 1.8$

$\mathrm{T} / \mathrm{K}=\left(\mathrm{t} /{ }^{\circ} \mathrm{F}+459.67\right) / 1.8$

$\mathrm{t} /{ }^{\circ} \mathrm{C}=\mathrm{T} / \mathrm{K} 2273.15$ kelvin $(\mathrm{K})$

degree Celsius $\left({ }^{\circ} \mathrm{C}\right)$

degree Celsius $\left({ }^{\circ} \mathrm{C}\right)$

kelvin $(\mathrm{K})$

kelvin $(\mathrm{K})$

meter per second $(\mathrm{m} / \mathrm{s})$

meter per second $(\mathrm{m} / \mathrm{s})$

meter per second $(\mathrm{m} / \mathrm{s})$

kilometer per hour $(\mathrm{km} / \mathrm{h})$

meter per second $(\mathrm{m} / \mathrm{s})$

$$
\begin{aligned}
& \text { cubic meter }\left(\mathrm{m}^{3}\right) \\
& \text { cubic meter }\left(\mathrm{m}^{3}\right) \\
& \text { cubic meter }\left(\mathrm{m}^{3}\right) \\
& \text { cubic meter }\left(\mathrm{m}^{3}\right) \\
& \text { liter }(\mathrm{L}) \\
& \text { cubic meter }\left(\mathrm{m}^{3}\right) \\
& \text { cubic meter }\left(\mathrm{m}^{3}\right) \\
& \text { milliliter }(\mathrm{mL})
\end{aligned}
$$

$1.0 \mathrm{E}+00$

$1.0 \mathrm{E}+00$

$5.555556 \mathrm{E}-01$

$5.555556 \mathrm{E}-0$

$5.555556 \mathrm{E}-01$

3.048 E-01

$2.54 \mathrm{E}-02$

2.777778 E-01

$1.609344 \mathrm{E}+00$

$2.68224 \mathrm{E}+01$

$2.831685 \mathrm{E}-02$

1.638706 E-05

7.645549 E-0 I

3.785412 E-03

$3.785412 \mathrm{E}+00$

$1.0 \mathrm{E}-03$

2.957353 E-05

$2.957353 \mathrm{E}+01$ 
This page intentionally left blank. 


\section{TABLE OF CONTENTS}

List of Tables

G-ii

\section{Appendix G}

Interim Report on Significant Fires in WTC 1, 2, and 7 Prior to September 11, 2001 ...... G-1

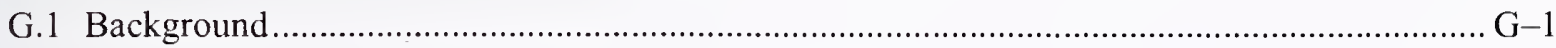

G.2 FDNY Fire Reports and Fire Investigation Reports ............................................................. G-1

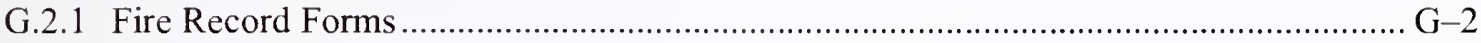

G.2.2 Overview of Fire Incidents 1970-2001 from FDNY Records .................................... G-3

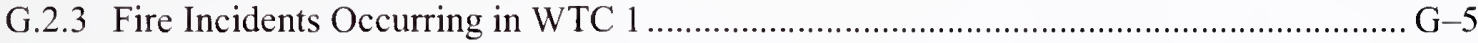

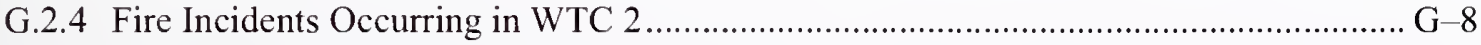

G.2.5 Additional Fires Involving the Deployment of Standpipe Lines in WTC 1 and $2 \ldots \ldots \ldots$. G-9

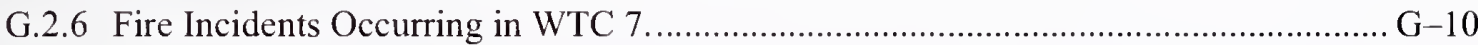

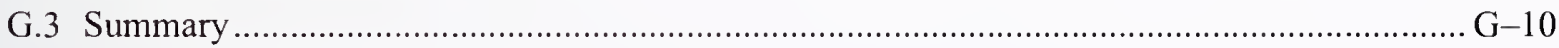

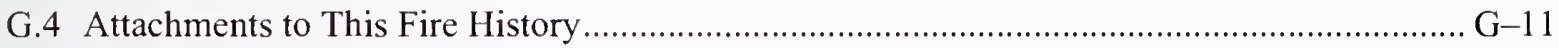

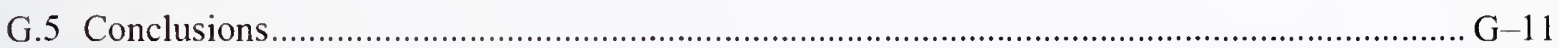

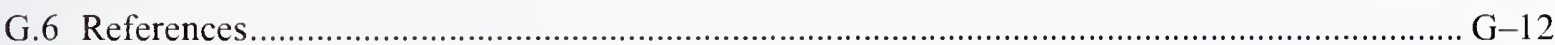

Attachments G-A.1 through G-A.3

Numeric Codes Used in the Fire Reports .................................................................. G-13

Attachments G-A.4 through G-A.7

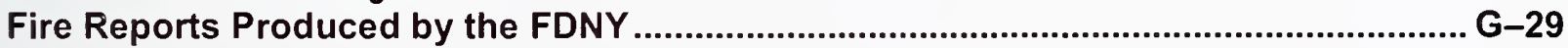




\section{LIST OF TABLES}

Table G-1. Categorization of WTC 1, 2, and 7 fires from FDNY records........................................... G-3

Table G-2. Significant fires in WTC 1 extinguished by sprinklers and/or multiple standpipe lines...... G-5

Table G-3. Significant fires in WTC 2 extinguished by sprinklers and/or multiple standpipe lines...... G-8

Table G-4. Significant fires in WTC 7 extinguished by sprinklers and/or multiple standpipe lines.... G-10 


\section{Appendix G \\ INTERIM REPORT ON SIGNIFICANT FIRES IN WTC 1, 2, AND 7 PRIOR TO SEPTEMBER 11, 2001}

Fires occurred in World Trade Center (WTC) 1, 2, and 7 prior to September 11, 2001. This appendix documents the facts of significant fires in the building after first occupancy as they relate to the performance of the automatic sprinkler, manual suppression, fire detection, and smoke purge systems. The ultimate goal of this review was to identify from New York City Fire Department (FDNY) records significant but not well known fires for further study.

\section{G.1 BACKGROUND}

The fire protection engineering department of the Port Authority of New York and New Jersey (PANYNJ) maintained records of all significant fire events in the WTC buildings. These records were lost in the collapse of the towers.

Two significant fire events involving WTC 1 are well known. On Februarry 14, 1975, a fire started on the 11 th floor of WTC 1 . Workers reported the fire to WTC police headquarters. When police reached the fire floor, they reported a serious fire and ordered the heating, ventilating, and air conditioning (HVAC) system be placed into the smoke purge mode. Fire spread through unprotected floor openings in utility closets. Fire damage occurred on floor 10 through floor 19 . Approximately $800 \mathrm{~m}^{2}\left(9,000 \mathrm{ft}^{2}\right)$ of the floor 11 contents were destroyed or damaged. At that time, sprinklers had not been installed in the office spaces. However, fire barriers divided the floor into quadrants. The fire on floor 11 was confined to the southeast quadrant. Fire damage on other floors was confined to the utility closets. The fire was extinguished by FDNY. More details about this fire incident can be found in Powers (1975), Lathrop (1975), and a report that is being prepared for the National Institute of Standards and Technology (NIST) by Hughes Associates.

At 12:18 PM on February 26, 1993, a bomb exploded in an underground parking garage of the WTC complex. The explosion occurred on the B2 level in the area of the garage under WTC 3 and adjacent to WTC 1 . The explosion resulted in a loss of normal electric power in WTC 1 and WTC 2. HVAC systems shut down. Smoke spread throughout WTC 1 and to a lesser extent in WTC 2. More details about this fire can be found in Isner and Klein (1993a, 1993b). The only historic record of smaller fire incidents in WTC 1,2, and 7 known to this investigation are the fire reports and fire investigation reports prepared by the FDNY. These reports were provided to NIST by FDNY for use in this investigation.

\section{G.2 FDNY FIRE REPORTS AND FIRE INVESTIGATION REPORTS}

The FDNY released 397 Bureau of Operations Fire Reports and 112 Bureau of Fire Investigation Records (Fire Marshals' Reports) which served as the basis for a summary of the fire history in the WTC 1, 2, and 7. NIST obtained reports of fires for the period of 1970-2001 and fire investigation records between 1977 and 2001 for WTC 1, 2, and 7, which in total, consisted of over 500 documents on which to report. 
These records included all responses to fires in buildings 1, 2, and 7 by the FDNY. All of these records consist of standardized forms that may be supplemented with other materials. Many were for minor fire events, such as fires that were extinguished by occupants before FDNY arrival. These were not of interest for this investigation. The records of significant fires were identified.

Significant fire incidents were those that exercised the fire suppression systems, specifically multiple sprinklers or multiple standpipes (with or without the activation of at least one sprinkler). These fires will be discussed individually, organized by the building in which they occurred. In addition to these fires, generalized facts relating to those fires involving the use of one standpipe line and one sprinkler and the use of one standpipe line will be provided throughout this report. As an aside, the majority of fire records for significant fires documented the performance of the detectors and sprinkler systems, but almost all reports lacked information about the performance of the smoke purge system.

\section{G.2.1 Fire Record Forms}

Depending upon the type and date of the incident, a specific fire report form was used by the FDNY to document the incident. For each type of emergency responded to by the FDNY, responders either completed a form that would describe a structural fire $(\mathrm{BF}-24)$ or a form that would describe any other type of emergency (BF-25), such as a nonstructural fire, transportation fire, and/or any other non-fire emergency. For this historical summary, only those events logged and organized under the structural fire form, 345 documents total, were of interest and used. A structural fire form is a one-page document (unless additional information is recorded on separate sheets) that gives valuable information about the fire event on various subjects, including:

- Alarm-the date and time of the received alarm

- Injuries and casualties-the numbers of each for the incident

- Extinguishment-details of the sprinkler and standpipe performance

- Ignition-information on the equipment involved in ignition, the form of the ignition source, the material type and form that was ignited, and the ignition factor (cause)

- Structure-information on the class of construction, the use of the building, and its status (vacant, occupied, under construction, etc.)

- Fire origin-the fire location and classification

- Fire extension-the means of fire extension and number of buildings/vehicles involved

- Damage-information on the damage done by flame, smoke, and water

- Detectors-the type, power source and performance of the detectors in the fire area

Each subject of the incident is given a set of codes or numbers that correspond to any incident, and in order to read the fire records successfully, an understanding of the codes is necessary (see 
Attachments G-A.1, G-A.2 and G-A.3). For the nonstructural B-25 record forms, the only fire related subjects included are the injury and casualty numbers, ignition, and structure information.

Depending upon the date of the fire incident, certain information is lacking from the structural fire form. Before 1980, a different record form for structural fire incidents was used which left out the following subjects: fire extension, damage, detectors, and portions of the ignition data. Because of this, detection data are not available for the majority of the fires occurring before 1980 .

\section{G.2.2 Overview of Fire Incidents 1970-2001 from FDNY Records}

Table G-1 contains the categorization of all structural fire incidents contained in the FDNY records for WTC buildings 1,2, and 7 available to this investigation. The table contains information on the category of fire incident (whether or not the detection and/or sprinkler systems activated), the time period over which the fires occurred, the numbers of records in that category, and a descriptive statement about the category.

Table G-1. Categorization of WTC 1, 2, and 7 fires from FDNY records.

\begin{tabular}{|c|c|c|c|}
\hline \multicolumn{4}{|c|}{ WTC 1} \\
\hline Category & Dates & Number & Generalization of Incidents \\
\hline $\begin{array}{l}\text { No detection, no } \\
\text { sprinkler }\end{array}$ & $1980-2001$ & 66 & $\begin{array}{l}\text { Unattended food/appliances, overheated elevator } \\
\text { equipment, discarded material, welding } \\
\text { operations, electrical failure and suspicious fires }\end{array}$ \\
\hline $\begin{array}{l}\text { No detection } \\
\text { information and no } \\
\text { sprinklers }\end{array}$ & $1970-1979$ & 79 & $\begin{array}{l}\text { Trash can fires, discarded material, food on stove, } \\
\text { electrical failure, overheated equipment }\end{array}$ \\
\hline Detection, no sprinklers & $1980-2000$ & 57 & $\begin{array}{l}\text { Unattended food/appliances, overheated elevator } \\
\text { equipment, discarded material, welding } \\
\text { operations, electrical failure }\end{array}$ \\
\hline $\begin{array}{l}\text { [Detection] and } \\
\text { sprinklers }\end{array}$ & $1977-1999$ & 18 & Suspicious, electrical failure, discarded material \\
\hline \multicolumn{4}{|c|}{ WTC 2} \\
\hline Category & Dates & Number & Generalization of Incidents \\
\hline $\begin{array}{l}\text { No detection, no } \\
\text { sprinkler }\end{array}$ & 1980-1999 & 37 & $\begin{array}{l}\text { Discarded material, welding too close, overheated } \\
\text { equipment, suspicious, elevator motor }\end{array}$ \\
\hline $\begin{array}{l}\text { No detection } \\
\text { information and no } \\
\text { sprinklers }\end{array}$ & $1975-1979$ & 40 & $\begin{array}{l}\text { Discarded material, fire in office furniture, trash } \\
\text { can fires }\end{array}$ \\
\hline Detection, no sprinklers & 1981-1999 & 40 & $\begin{array}{l}\text { Food on stove, small elevator fire, electrical } \\
\text { failure, suspicious, overheated equipment }\end{array}$ \\
\hline $\begin{array}{l}\text { [Detection] and } \\
\text { sprinklers }\end{array}$ & $1977-2000$ & 5 & Mechanical failure, suspicious \\
\hline \multicolumn{4}{|c|}{ WTC 7} \\
\hline Category & Dates & Number & Generalization of Incidents \\
\hline $\begin{array}{l}\text { No detection, no } \\
\text { sprinkler }\end{array}$ & 2000 & 1 & Trash can fire/discarded material \\
\hline Detection, no sprinklers & 1990 & 1 & Electrical switch on floor - explosion \\
\hline $\begin{array}{l}\text { [Detection] and } \\
\text { sprinklers }\end{array}$ & 1988 & 1 & Suspicious \\
\hline
\end{tabular}


All FDNY records provided to NIST, unless the records were not readable, contained relevant information about the type and performance of the suppression system. Because of this, reports of incidents in which the sprinkler system activated can range from 1970 to 2001 . When the table lists "[detection]" in brackets, this is meant to symbolize that either detection was present or no information on detector performance was included on the form (as is the case with the older records). An attempt was made to compare all investigation records with the fire reports, especially those which activated the suppression system. Looking at the records in Table G-1, it is clear that only 24 fires activated the sprinkler system from 1970-2001 from all three buildings. Many of the other structural fires without sprinkler activation were labeled as suspicious, trash can fires, electrical failures, unattended food/appliances, or overheated equipment.

In order to report on significant structural fires occurring in WTC 1, 2, and 7, the FDNY records had to be reviewed for those incidents that activated sprinklers, detectors, or were extinguished by hose line and those smaller fires that self-extinguished or could be extinguished using a fire extinguisher. The structural fire incidents without detection information (before 1980), had to be reviewed to locate any fires that activated the sprinkler system.

The retrofit installation of sprinklers into WTC buildings 1 and 2 was accomplished in two phases. During the first phase in 1976, sprinkler risers/mains were installed throughout WTC 1 and WTC 2. Sprinklers were installed to protect corridors, storage rooms, lobbies, and certain tenant/PANYNJ spaces. In the second phase of the retrofit from 1983 to 2001, sprinklers were installed in all remaining places in the complex (PACO 2002; shown in Attachment G-B). Prior to the retrofit only the sub-grade areas and selected hazard areas were protected by automatic sprinklers. This retrofit proceeded throughout the buildings as much as practical when other renovations of the office spaces were underway, such as when change of tenants occurred.

After the installation of the sprinkler risers in 1976, tenants had the option of providing sprinklers or compartmentation for fire protection in compliance with Local Law 5. It was therefore possible that during the period of time when retrofit installation of sprinklers was under way, a fire that occurred may or may not have been in an area protected by automatic sprinklers.

The forms used by the FDNY after 1987 give a detailed description of the event and whether or not a system was present at the time of the fire; however, a fire recorded before 1987 will give data only on the number of sprinklers opened. Because of this, an effort was made to look through all reports, especially those that mentioned detection performance, in order to identify fires involving the use of standpipe lines by the FDNY as an alternate indication of a significant fire.

The next section of the report will highlight significant fires occurring in WTC 1,2, and 7. The significant fires will be described individually by WTC building, and organized by the date on which they occurred in the building. In addition to these significant fires, (1) the fires that activated one sprinkler head and involved the use of one standpipe and (2) the fires that involved the use of only one standpipe, due to the number of incidents, will be generalized as to the nature of the incidents and the procedures followed by the FDNY. 


\section{G.2.3 Fire Incidents Occurring in WTC 1}

After reviewing all the FDNY records of fire incidents in WTC building 1 since 1970, the significant fires were selected. There were 12 significant fires found for WTC 1 , and the fire reports are included in Attachment G-A.4. Table G-2 provides a summary of the fire incident information from FDNY records, which is followed by individual paragraphs about each incident.

\section{Table G-2. Significant fires in WTC 1 extinguished by sprinklers and/or multiple} standpipe lines.

\begin{tabular}{|c|c|c|c|c|c|c|}
\hline $\begin{array}{l}\text { Significant } \\
\text { Fire }\end{array}$ & $\begin{array}{l}\text { Incident } \\
\text { Date }\end{array}$ & Fire Location & $\begin{array}{c}\text { \# Sprinklers } \\
\text { Activated }\end{array}$ & $\begin{array}{c}\text { \# Standpipes } \\
\text { Used }\end{array}$ & Cause of Fire & $\begin{array}{l}\text { Material } \\
\text { Ignited }\end{array}$ \\
\hline 1 & $9 / 9 / 77$ & $\begin{array}{l}\text { B-6 level storage } \\
\text { room }\end{array}$ & 2 & 0 & None listed & Not listed \\
\hline 2 & $9 / 23 / 77$ & $\begin{array}{l}\text { Dumpster on B-4 } \\
\text { level }\end{array}$ & 2 & 0 & $\begin{array}{l}\text { Not } \\
\text { classified }\end{array}$ & Trash/waste \\
\hline 3 & $10 / 16 / 81$ & 19th floor office area & - & 2 & $\begin{array}{l}\text { Discarded } \\
\text { material }\end{array}$ & Furniture \\
\hline 4 & $12 / 23 / 83$ & $\begin{array}{l}2 \text { dumpsters on B-4 } \\
\text { level }\end{array}$ & 2 & 1 & Suspicious & Trash/waste \\
\hline 5 & $1 / 27 / 85$ & $\begin{array}{l}\text { Office space on } \\
\text { mezzanine level } \\
\text { (Floor } 2)\end{array}$ & 2 & 1 & Incendiary & Trash/waste \\
\hline 6 & $9 / 10 / 85$ & $\begin{array}{l}\text { Garbage dumpster in } \\
\text { service elevator } \\
\text { lobby on floor } 43\end{array}$ & 2 & 1 & Suspicious & Trash/waste \\
\hline 7 & $11 / 1 / 85$ & $\begin{array}{l}\text { Storage closet on B-4 } \\
\text { level }\end{array}$ & 3 & 1 & Suspicious & $\begin{array}{l}\text { Supplies/ } \\
\text { stock }\end{array}$ \\
\hline 8 & $6 / 7 / 86$ & $\begin{array}{l}\text { Dumpster fire on } \\
\text { floor } 106, \text { compactor } \\
\text { room on floor } 107\end{array}$ & 2 & 1 & None listed & Trash/waste \\
\hline 9 & $9 / 30 / 91$ & Office on B-4 level & $\geq 1$ & 2 & $\begin{array}{l}\text { Discarded } \\
\text { material }\end{array}$ & Trash/waste \\
\hline 10 & $11 / 19 / 91$ & $\begin{array}{l}\text { Electrical closet on } \\
\text { floor } 93\end{array}$ & 0 & 2 & Short circuit & $\begin{array}{l}\text { Electrical } \\
\text { wire or cable } \\
\text { insulation }\end{array}$ \\
\hline 11 & $7 / 23 / 92$ & $\begin{array}{l}\text { Level B-5 at the } \\
\text { power distribution } \\
\text { panel }\end{array}$ & 0 & 2 & $\begin{array}{l}\text { Electrical } \\
\text { failure }\end{array}$ & $\begin{array}{l}\text { Electrical } \\
\text { wire or cable } \\
\text { insulation }\end{array}$ \\
\hline 12 & $11 / 10 / 99$ & $\begin{array}{l}\text { Computer room on } \\
\text { floor } 104\end{array}$ & 3 & $\geq 1$ & None listed & $\begin{array}{l}\text { Plastics, } \\
\text { electronic } \\
\text { equip }\end{array}$ \\
\hline
\end{tabular}

Key: $\geq$ symbol denotes that at least one of the units of the suppression system was used (and not specifically identified by the fire report); - indicates that the report acknowledges 0 sprinklers open; however, due to the date of the fire, the space may not have had a sprinkler system installed. 


\section{Significant Fire \#1}

On September 9, 1977, at 11:04 p.m., the FDNY received an alarm for a fire in the B-6 level storage room at the address of WTC 1 . The fire activated two sprinklers, and was noted to be extinguished before the FDNY's arrival.

\section{Significant Fire \#2}

Another fire occurred on September 23, 1977, at 11:48 p.m., in a dumpster on the B-4 level of WTC 1. This fire also activated two sprinklers, and the FDNY noted that the fire had been extinguished prior to their arrival.

In both cases, no injuries or casualties resulted from these fires, and the damage was confined to the area of origin.

\section{$\underline{\text { Significant Fire \#3 }}$}

Six years later, on October 16, 1981, at 7:12 p.m., a fire occurred on floor 19 of WTC 1. The FDNY noted that they used two standpipe lines to extinguish the fire and that one person was evacuated from the scene. Again, the fire report notes that no sprinklers opened, but does not note whether or not sprinklers were present at the time of the fire. Given the date of the incident, sprinklers are not expected to be located on floor 19. The fire was caused by discarded material and involved furniture in an office area of the floor.

\section{$\underline{\text { Significant Fire \#4 }}$}

Six years later on December 23, 1983, at 2:50 a.m., the FDNY responded to an alarm of fire and heavy smoke conditions on the B-4 level of WTC 1. The FDNY found two dumpsters fully involved in separate locations on the same floor and noted that the two activated sprinklers extinguished a major portion of the fire. The FDNY extinguished the rest of the flames by stretching hose from the standpipe system. Again, no injuries or casualties resulted from this fire. The cause noted on the report was suspicious and the damage was confined to the origin of the fire.

\section{$\underline{\text { Significant Fire \#5 }}$}

On January 27,1985 , at 8:53 p.m., the FDNY was called for a fire located in an unoccupied office on the mezzanine level of WTC 1 . Two sprinklers contained the incendiary (involving arson) fire consuming trash paper/waste. When the FDNY arrived, they extinguished the remaining fire with one standpipe line. Building and content damage was confined to less than 15 percent of the space. Also, no injuries or casualties were reported.

\section{$\underline{\text { Significant Fire \#6 }}$}

Eight months later on September 10, 1985, at 4:05 p.m., the Port Authority Police informed the FDNY on arrival of a sprinkler flow and smoke condition on floor 43. A medium smoke condition was report by the FDNY on floor 43, where a fire was extinguished by two sprinklers. The fire report notes the use of one standpipe line; however, this was used during the overhaul process. This fire originated suspiciously 
in a garbage dumpster in a service elevator lobby. There was no building or content damage as well as no injuries or casualties reported.

\section{$\underline{\text { Significant Fire \#7 }}$}

On November 1, 1985, at 4:05 a.m., the FDNY was called for another suspicious fire producing heavy smoke on the B-4 level under WTC 1 and WTC 2. This fire occurred in a storage closet of the men's bathroom, and the FDNY noted that three sprinklers activated to keep the fire under control until their arrival. Upon arrival, the FDNY extinguished the remaining fire in the closet area with one standpipe line. Again, the damage was noted to be confined to the area of origin.

\section{$\underline{\text { Significant Fire \#8 }}$}

Less than a year later, on June 7,1986, at 9:49 a.m., the FDNY received an alarm for a heavy smoke condition on floor 110. For this call, fires were burning in two separate places; a garbage dumpster on floor 106 and the compactor room on floor 107. Sprinklers were noted in operation in both locations and seemed to control the fires, until the FDNY could complete extinguishment with one standpipe line on floor 106. There was no report of injuries or casualties for the previous two fires.

\section{$\underline{\text { Significant Fire \#9 }}$}

An additional fire occurred in WTC 1 where multiple standpipe lines were used along with the activation of the sprinkler system. This fire occurred on September 30, 1991, at 6:32 p.m., in an office on the B4 level. The fire report noted that the sprinkler system operated; however, there is no mention of how many sprinklers or even their activation in the Operations/Comments section of the report. Two $13 / 4 \mathrm{in}$. or larger hose lines were used by the FDNY to extinguish this fire. The cause of the fire was abandoned material (cigarette) igniting boxes/carton material in an office. The fire damage was confined to the area of origin and smoke damage was confined to the floor. There was one uniformed officer injured and no civilian injuries or casualties.

\section{$\underline{\text { Significant Fire \#10 }}$}

A fire occurred on November 19, 1991, at 6:27 pm., and two 2 1/2 in. standpipe hose lines were used by the FDNY. The FDNY responded to WTC 1 for this fire due to a report of fire and smoke condition in electrical closets on possibly four floors (floors 93-96) and an alarm transmitted from floors 93-98. According to the fire report, the sprinklers were in service, but did not operate for this fire. The noted cause of this fire was a short circuit and the material that was ignited was electrical wire or cable insulation. The fire and smoke damage was confined to its area of origin (electrical closet). Two occupants were removed from stalled elevators during this incident, and occupants were evacuated from the scene, although an exact number is not given. Also, two occupants were injured and required first aid.

\section{$\underline{\text { Significant Fire \#11 }}$}

The FDNY responded to WTC 1 on July 23, 1992, at 10:02 p.m., due to a transformer fire on the 5th sub basement level. Firefighters found a fire situation in a large power distribution panel, where a firefighter was knocked unconscious by a shock blast from the panel. Similar to the fire in November of 1991, two $2-1 / 2$ in. standpipe hose lines were used by the FDNY on this fire. The cause of the fire was an electrical 
failure and the material ignited was electrical wire or cable insulation. No appreciable damage is noted. As mentioned earlier, one firefighter was injured as well as three civilians.

\section{$\underline{\text { Significant Fire \#12 }}$}

The final fire associated with WTC 1 was one that occurred on November 10, 1999, at 11:01 p.m., in a computer room on floor 104. The FDNY noted that the fire was "knocked down" by three sprinklers when they arrived and they completed extinguishment with a line extended from the standpipe. The flame damage was confined to the area of origin and computer equipment was involved in fueling the fire. There was one injury and no casualties reported in the FDNY record for this fire.

Table G-2 presents the 12 significant fires in WTC 1. Five of the 12 fires occurred on the basement levels and two occurred on the upper levels (above floor 100). The causes of these significant fires include suspicious, discarded materials, and electrical failures.

\section{G.2.4 Fire Incidents Occurring in WTC 2}

Table G-3 presents the significant fire occurring in WTC 2. There were three significant fires found for WTC 2, and the fire reports are included in Attachment G-A.5. Table G-3 provides a summary of the fire incident information from FDNY records, which is followed by individual paragraphs about each incident.

Table G-3. Significant fires in WTC 2 extinguished by sprinklers and/or multiple standpipe lines.

\begin{tabular}{|c|c|l|c|c|c|c|}
\hline $\begin{array}{c}\text { Significant } \\
\text { Fire }\end{array}$ & $\begin{array}{c}\text { Incident } \\
\text { Date }\end{array}$ & Fire Location & $\begin{array}{c}\text { \# Sprinklers } \\
\text { Activated }\end{array}$ & $\begin{array}{c}\text { \# Standpipes } \\
\text { Used }\end{array}$ & $\begin{array}{c}\text { Cause of } \\
\text { Fire }\end{array}$ & $\begin{array}{c}\text { Material } \\
\text { Ignited }\end{array}$ \\
\hline 1 & $5 / 19 / 75$ & Floor 32 & - & 3 & Incendiary & Trash/waste \\
\hline 2 & $4 / 12 / 77$ & $\begin{array}{l}\text { Duct work over } \\
\text { grill in } \\
\text { restaurant on } \\
\text { floor 107 }\end{array}$ & 2 & 0 & None listed & Duct work \\
\hline 3 & $3 / 22 / 93$ & $\begin{array}{l}\text { Fan motor room } \\
\text { on floor 108 }\end{array}$ & 2 & 0 & $\begin{array}{l}\text { Mechanical } \\
\text { failure }\end{array}$ & $\begin{array}{c}\text { Not } \\
\text { classified }\end{array}$ \\
\hline
\end{tabular}

- Indicates that the report acknowledges 0 sprinklers open, however due the date of the fire, the space may not have had a sprinkler system installed.

\section{$\underline{\text { Significant Fire \#1 }}$}

A fire occurred on May 19, 1975, at 9:38 p.m., on floor 32 of WTC 2. The FDNY noted that they used three standpipe lines to extinguish the fire and that the Port Authority reported occupants trapped on floors 31 and 32. The fire report notes that no sprinklers opened, but does not note whether or not sprinklers were present at the time of the fire. Given the date of the incident, sprinklers are not expected to be located on floors 31 and 32. The fire was labeled as incendiary and involved trash/waste. The FDNY stated that the fire involved the core area of the floor and was confined to that area. Over 20 people (civilians and uniformed personnel) were injured by this incident. 


\section{$\underline{\text { Significant Fire \#2 }}$}

On April 4, 1977, at 1:15 p.m., the FDNY was called to WTC 2 for a fire in the duct work over the grills in a restaurant on floor 107. The FDNY record on this fire noted that the fire was extinguished prior to its arrival. The damage was confined to the area of origin, and the fire caused no injuries or casualties.

\section{$\underline{\text { Significant Fire \#3 }}$}

The second fire occurred on March 22, 1993, at 8:39 a.m., and caused a smoke condition on floor 108 . The fire activated two sprinklers due to an overheated bearing in a fan motor room on floor 108 . The damage to the area did not exceed 15 percent of the space, and there were no injuries or casualties reported.

Table G-3 presents the three significant fires in WTC 2. No fires were discovered in WTC 2 where multiple sprinklers or standpipes were used with another suppression system. Two of the three fires occurred on the upper levels (above floor 100) and the other occurred on floor 32. The causes of these significant fires included incendiary and mechanical failures.

\section{G.2.5 Additional Fires Involving the Deployment of Standpipe Lines in WTC 1 and 2}

The fires described in this section (31 in total) involve the use of one standpipe, with and without the activation of one sprinkler for WTC 1 and WTC 2. Four of the 31 reports describe fires that were extinguished with one sprinkler and one standpipe line (see Attachment G-A.6.1). Three of these fires were located in WTC 1 between the years of 1986-1991 and the other in WTC 2 in 1981. Two of these fires occurred in basement levels, one occurred on floor 106 of WTC 1, and the last on floor 5 in WTC 1. 1n some of the fire reports, the FDNY noted that the sprinkler controlled the fire, and the standpipe was used to actually extinguish the remaining fire. Half of the fires were labeled as incendiary/suspicious, one was an electrical failure, and the last was unknown.

In addition, 27 of the 31 fire reports describe fires that were extinguished using one standpipe line (see Attachment G-A.6.2). Twenty of these fires occurred in WTC 1 and the other seven occurred in WTC 2. A majority of these fires (19) are labeled as incendiary/suspicious or unknown, while the other causes of the fires are attributed to short circuits, abandoned material/cigarette, welding close to combustibles, and a mechanical failure. The dates of occurrence for these fires range from 1973-1999, with a majority (23) occurring between the years of 1973-1985. These fire incidents did not result in any casualties, but five civilians and one uniformed officer were injured.

Two of the 27 fires involved a 300 person (April 19, 1980) and a 1,500 person (April 17, 1981) evacuation. These will be described in further detail. On April 19, 1980, at 2:06 p.m., the FDNY received reports of an activated smoke detector in the return air duct on floor 106 of WTC 1. The FDNY also received reports of heavy smoke on floor 106, light smoke on floor 109, and heavy odor of smoke in stairways A and B. The report notes that while only one standpipe was used, approximately 300 people were evacuated from the Windows on the World restaurant on floor 107 via stairway $\mathrm{C}$ (which was clear of smoke). The fire cause was labeled as abandoned or discarded material and involved plastic material. This fire did not cause any injuries or casualties. 
On April 17, 1981, at 9:18 a.m., the FDNY was informed of a fire on floor 7 and a smoke condition on floors 7 through 11 of WTC 1 . The FDNY hooked up one standpipe and extinguished the fire located in an air conditioning unit in the "MER" room on floor 7. The cause of this fire was labeled as a mechanical failure. The fire report notes that the Port Authority personnel reported an evacuation of approximately 1,500 people from floors 9 through 23 . However, no injuries or casualties were reported from this fire.

\section{G.2.6 Fire Incidents Occurring in WTC 7.}

Table G-4 presents the significant fire occurring in WTC 7. There was one significant fire found for WTC 7, and the fire report is included in attachment $\mathrm{G}-\mathrm{A}$.7. Table 4 provides a summary of the fire incident information from FDNY records, which is followed by an individual paragraph on the incident.

Table G-4. Significant fires in WTC 7 extinguished by sprinklers and/or multiple standpipe lines.

\begin{tabular}{|c|c|c|c|c|c|c|}
\hline $\begin{array}{c}\text { Significant } \\
\text { Fire }\end{array}$ & $\begin{array}{c}\text { Incident } \\
\text { Date }\end{array}$ & Fire Location & $\begin{array}{c}\text { \# Sprinklers } \\
\text { Activated }\end{array}$ & $\begin{array}{c}\text { \# Standpipes } \\
\text { Used }\end{array}$ & Cause of Fire & $\begin{array}{c}\text { Material } \\
\text { Ignited }\end{array}$ \\
\hline 1 & $5 / 20 / 88$ & $\begin{array}{c}\text { Construction shanties } \\
\text { on floor } 3\end{array}$ & $\begin{array}{c}\text { Multiple, \# not } \\
\text { listed }\end{array}$ & 1 & Suspicious & Shanties \\
\hline
\end{tabular}

\section{$\underline{\text { Significant Fire \#1 }}$}

In WTC 7, a fire occurred on May 20,1988, at 12:38 a.m., in the construction shanties on floor 3. Although the fire report does not specifically note the number of sprinklers that activated, the operations notes state that Ladder Truck 10 found the sprinklers (noting more than one) in operation and shut them down. The FDNY had to complete the extinguishment by stretching a line from the standpipe to the fire source. This fire is noted by the report as being suspicious in nature and the flame damage was confined to the area of origin.

It is possible that the fire incidents that were not specifically highlighted, especially those in the areas without sprinklers, involved other methods of extinguishment before FDNY arrival, such as a WTC houseline (pre-connected standpipe hose), hand extinguisher, or bucket of water, as noted on some of the FDNY reports. All other fires, the majority, included in other categories were either self-extinguished, extinguished prior to FDNY arrival (by staff, etc.), or a hand extinguisher was used by the FDNY.

\section{G.3 SUMMARY}

In summary, 16 significant fires occurred in WTC 1,2 , and 7, with 12 occurring in WTC 1 , three in WTC 2 , and one in WTC 7. In addition to these, 31 fires occurred in WTC 1 and WTC 2, which involved the use of one standpipe (with or without the activation of one sprinkler). Of these additional 31 fires, 23 occurred in WTC 1 and eight occurred in WTC 2. The following paragraphs will summarize findings from the 16 significant fires that occurred in all three buildings.

After reviewing the 16 significant fires, trends developed relating to the time of day that the fires occurred. Overall, 12 of the 16 fires occurred between the hours of 6 p.m. and 4 a.m. The fires that occurred during office hours (between 7 a.m. and 6 p.m.) included a dumpster fire in the floor 43 elevator lobby (WTC 1), a dumpster fire on floor 106 (WTC 1), a kitchen fire on floor 107 (WTC 2), and a bearing overheating in the fan motor room on floor 108 (WTC 2). Almost all of the incendiary (arson) and 
suspicious fires (5 out of 6 fires) and unclassified or unlisted fires (4 out of 5 fires) occurred after business hours (before 7 a.m. and after 6 p.m.).

In addition to the time of day of the fire, trends in the cause of the fire and the materials involved in the fire can be highlighted. Of the 16 fires and their causes, five were labeled as unlisted or unclassified, six as suspicious or incendiary, two as discarded material, and three as an electrical failure or mechanical failure. For the material involved in the fire, eight reports noted trash, waste, and supplies; two reported not listed or not classified; one reported furniture; three reported electrical equipment; one reported duct work; and one reported shanties were the material involved in the fire.

Lastly, the location of the fires throughout the buildings was of interest. Of the 16 fires, four fires were concentrated above floor 100 or and six fires were located in the basement. The others (6 fires) were spread throughout the rest of the building.

\section{G.4 ATTACHMENTS TO THIS FIRE HISTORY}

Attachments G-A.1 through G-A.7 are included as a supplement to this report. The first three sections, G-A.1 through G-A.3 are explanations of the numeric codes used in the fire reports by the FDNY. Attachment G-A.1 is included to explain the codes for the fire reports produced prior to and including 1980, Attachment G-A.2 is included to explain the fire reports produced from 1981 to May 31, 1987, and Attachment G-A.3 is included to explain the fire reports produced from June 1, 1987, to the present. The report code explanations are divided into the same sections as the fire report and give short descriptions for the numbers used in the fire report under each section. For example, if the ignition factor for a fire occurring in 1990 was given a number code of 54 , the reader can find that the cause of the fire was a "short circuit, ground fault."

Attachments G-A.4 through G-A.7 include the actual fire reports produced by the FDNY on the significant fires highlighted in the sections above. The reader can use Attachments G-A.1 through G-A.3 (depending upon the date of the fire) to read the fire reports in more detail than what is provided in this fire history report.

\section{G.5 CONCLUSIONS}

From the information contained in FDNY fire reports and fire investigation records provided to NIST, 47 fires occurred in WTC building 1, 2, and 7 that were of sufficient size and duration to activate multiple sprinklers or were estimated by NIST to be capable of doing so, over the time period the buildings were occupied. This total does not include the major 1975 office fire in WTC 1 or the 1993 bombing.

The records indicate that in areas protected by automatic sprinklers, no fire activated more than 3 sprinklers. Three sprinklers would provide coverage for a floor area of approximately $63 \mathrm{~m}^{2}\left(675 \mathrm{ft}^{2}\right)$. This area is much smaller than the $800 \mathrm{~m}^{2}\left(9,000 \mathrm{ft}^{2}\right)$ damaged by the 1975 fire in an office space unprotected with automatic sprinklers.

Many of the fires that occurred were recorded as suspicious or unknown in cause, occurred during offpeak work hours, and involved materials such as trash or paper-based supplies. In cases where sprinklers 
were activated, the FDNY records indicated that the sprinklers either extinguished the fire completely or aided in controlling the spread.

\section{G.6 REFERENCES}

Isner, M. S., and T. J. Klem. 1993a. Explosion and fire disrupt World Trade Center. NFPA Journal. National Fire Protection Association. 91-104.

Isner, M. S., and T. J. Klem. 1993b. World Trade Center Explosion and Fire, New York, New York, February 26, 1993. Fire Investigation Report. National Fire Protection Association. Quincy, MA.

PACO Group. 2002. World Trade Center General Description of All Building Systems and the Capital Program. August.

Powers, W. R. 1975. One World Trade Center Fire New York, N.Y., Febrnary 13, 1975. National Fire Protection Association. Boston, MA. 1-15. 


\section{Attachment G-A.1}

Explanation of Numeric Codes Used on Fire and Emergency Reports -

Prior to 1980 
CIASSIFICATION OP BOILDING gY USE

COMERCIAL

\begin{tabular}{|c|c|}
\hline & \\
\hline $\begin{array}{l}02 \\
02 \\
03 \\
06 \\
05 \\
06\end{array}$ & $\begin{array}{l}\text { Bank } \\
\text { Brewery } \\
\text { Coal Pocket } \\
\text { Department Store } \\
\text { Electric Power Plant } \\
\text { Pactory: Hulti oxc- }\end{array}$ \\
\hline 07 & $\begin{array}{l}\text { pancy } \\
\text { Pactory: single } \\
\text { Occupanicy }\end{array}$ \\
\hline $\begin{array}{l}08 \\
09 \\
10\end{array}$ & $\begin{array}{l}\text { Foundry } \\
\text { Freight Mepot } \\
\text { Garage: Non-Storage }\end{array}$ \\
\hline $\begin{array}{l}11 \\
12 \\
13\end{array}$ & $\begin{array}{l}\text { Storage } \\
\text { Gas Works } \\
\text { Lumber Yara }\end{array}$ \\
\hline 14 & $\begin{array}{l}\text { Hotor vehicle pepair } \\
\text { Shop }\end{array}$ \\
\hline $\begin{array}{l}15 \\
16 \\
17 \\
18\end{array}$ & $\begin{array}{l}\text { Office Building } \\
\text { Oil Selling station } \\
\text { Oil storage Plant } \\
\text { Pler, Wharve, Dock, } \\
\text { Bulkhead Building }\end{array}$ \\
\hline $\begin{array}{l}19 \\
20\end{array}$ & $\begin{array}{l}\text { Restaurant, Diner } \\
\text { shed, Newsstand, } \\
\text { shanty }\end{array}$ \\
\hline 21 & $\begin{array}{l}\text { Shipyard, Drydock } \\
\text { Stable }\end{array}$ \\
\hline 23 & $\begin{array}{l}\text { Stean cenerating } \\
\text { plant }\end{array}$ \\
\hline 24 & $\begin{array}{l}\text { store Building, } \\
\text { Taxpayer }\end{array}$ \\
\hline 25 & $\begin{array}{l}\text { Warehouse, store- } \\
\text { hotss }\end{array}$ \\
\hline 26 & $\begin{array}{l}\text { store bullding } \\
\text { Private Drelling }\end{array}$ \\
\hline 39 & other Comereial \\
\hline \multicolumn{2}{|c|}{ PU므. IC } \\
\hline 40 & $\begin{array}{l}\text { Alruort Buizeling } \\
\text { A-ylum }\end{array}$ \\
\hline $\begin{array}{l}42 \\
43 \\
44 \\
45\end{array}$ & $\begin{array}{l}\text { Bridge } \\
\text { Bus Terminal } \\
\text { Church, Synagogue } \\
\text { Dance Hall, Ban- } \\
\text { pet Hall }\end{array}$ \\
\hline $\begin{array}{l}46 \\
47\end{array}$ & $\begin{array}{l}\text { Dispensary, Clini= } \\
\text { Ferry Terminal }\end{array}$ \\
\hline & $\begin{array}{l}\text { Covernment Building } \\
\text { (Not othervise class- } \\
\text { if ied): }\end{array}$ \\
\hline $\begin{array}{l}48 \\
19\end{array}$ & $\begin{array}{l}\text { City } \\
\text { Intersate }\end{array}$ \\
\hline $\begin{array}{l}19 \\
50\end{array}$ & $\begin{array}{l}\text { Intersate } \\
\text { Federal }\end{array}$ \\
\hline $\begin{array}{l}51 \\
52 \\
53\end{array}$ & $\begin{array}{l}\text { Foreign } \\
\text { state } \\
\text { Hospital, Infirman }\end{array}$ \\
\hline $\begin{array}{l}53 \\
54 \\
55\end{array}$ & $\begin{array}{l}\text { Hospital, Infirmary } \\
\text { Nursing Home } \\
\text { Railroad Station }\end{array}$ \\
\hline 56 & $\begin{array}{l}\text { School: college. } \\
\text { University }\end{array}$ \\
\hline $\begin{array}{l}57 \\
58 \\
59\end{array}$ & $\begin{array}{l}\text { Private High } \\
\text { Public High } \\
\text { Puble Jurior } \\
\text { High }\end{array}$ \\
\hline 60 & $\begin{array}{l}\text { - Private Ele- } \\
\text { mentary }\end{array}$ \\
\hline 61 & $\begin{array}{l}\text { school: Public } \\
\text { Elcmentary }\end{array}$ \\
\hline 62 & $\begin{array}{l}\text { Children's } \\
\text { Narsery }\end{array}$ \\
\hline $\begin{array}{l}63 \\
64 \\
65 \\
66\end{array}$ & $\begin{array}{l}\text { Other } \\
\text { Television s'idio } \\
\text { Theatre, Leg biatiate } \\
\text { Theatre, Motion } \\
\text { Picture }\end{array}$ \\
\hline 67 & $\begin{array}{l}\text { Transit Systed - } \\
\text { Station Structure }\end{array}$ \\
\hline $\begin{array}{l}68 \\
69\end{array}$ & $\begin{array}{l}\text { Tunnel } \\
\text { Other Public }\end{array}$ \\
\hline
\end{tabular}

\section{RESIDERTIAL}

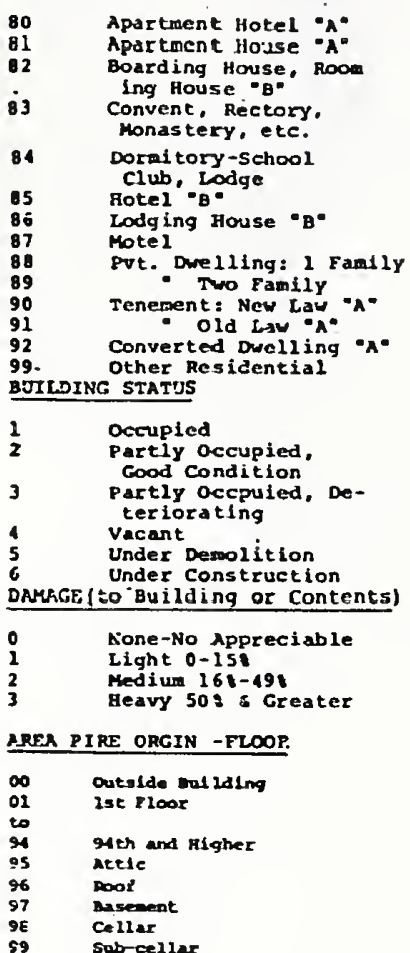

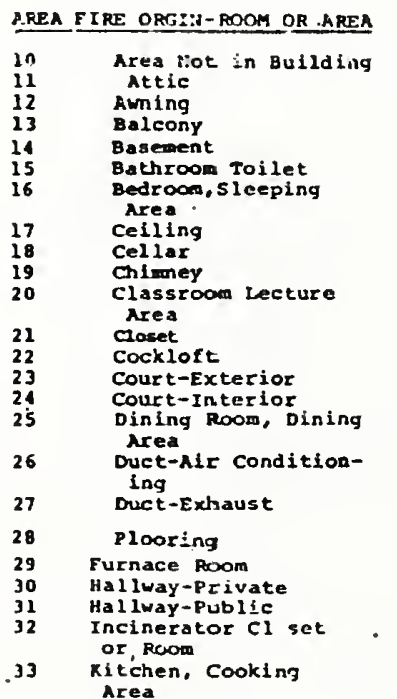

(2) ivirig Room

Lobry

Machinery Poon

Office Area

Operating Laboratory

Area

Partition

Porch

Projection Bootr

Recreation area

Roof

Sales Showroom Dis-

play Area

Sha:t-Duct, Pipe

Shaf $t$-Dumbwaiter

Shaft-Elevator

Shaft-Exterioz Light

shaft-Interior Light

Shaft-vent

Shipping Receiving

Stage

stalnay

Storage Ploom Area

Vacant-Roor, Apart-

ment or ared

6 Work Area Horkroo

Oher Areas. Not

iassif

AREA PIRE ORIGIN-OCCUPANCX

CIASSIFICATION

COMOERCIAL

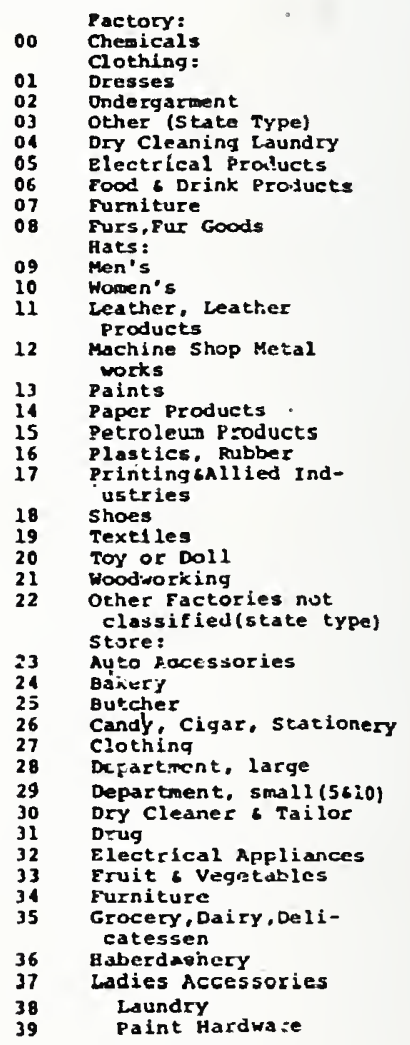




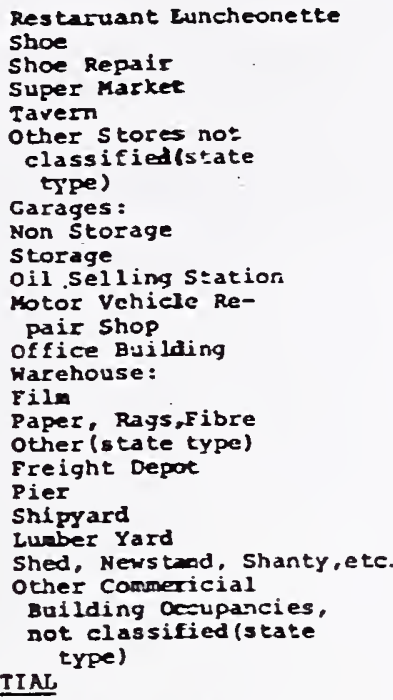

09 Shaft-Air,light,
10 Chute, Duct, ete.
11 Celling
12 Othdor (state how.
OLASSIPICATION BY TYPE FIRE
OR EMERGENCY
TRANSPORTATION FI ZES

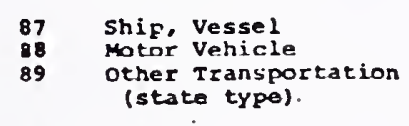

NON-STRUCTURAL SIFES

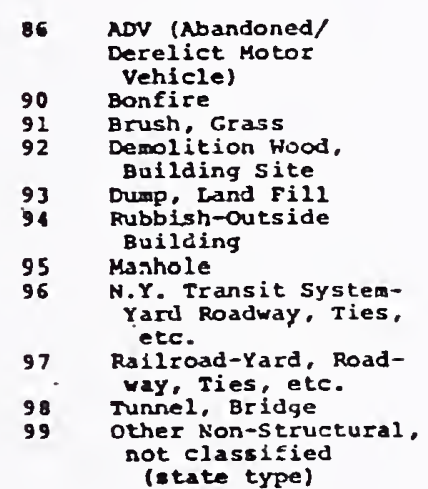

\section{EMERGENCY}

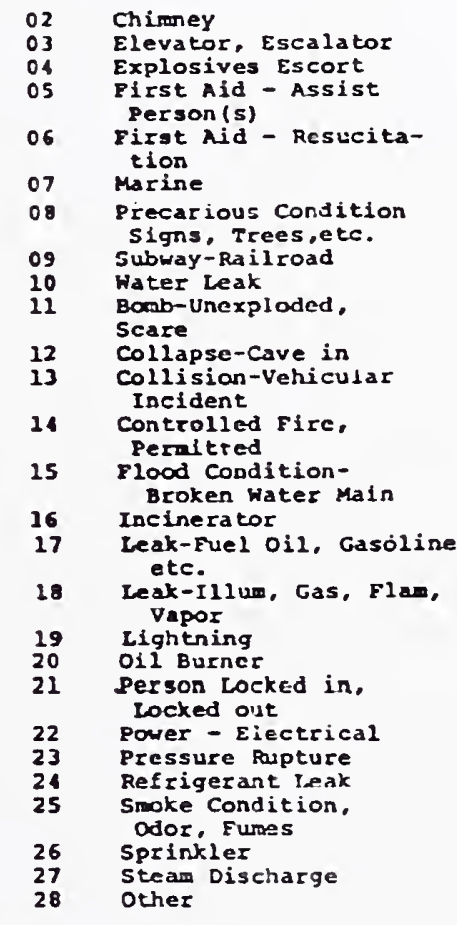


This page intentionally left blank 


\section{Attachment G-A.2}

Explanation of Numeric Codes Useḍ on Fire and Emergency Reports -

From 1981 to May 31, 1987 
Tiph of hepart

1 Sinctura.

Trasisoriation fú

3 Wos Sirvelyat Fate

4 Emerganicy Hespara?.

5 False Airm.

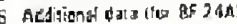

How Feportod

1 Sacel Bo Manual.

2 TElephas?

3 Verst.

4 Class 3-MEMU:. PFA

5. Cluss J-Yatus. PFA.

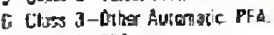

3 Clas; 3-EAS

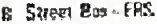

g. Chas 3-Moruss golpy.

19) Pre-recordud Alem

Ioitiol Aberim

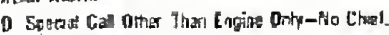

i Bor stimat in closs 3

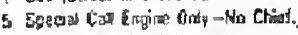

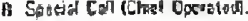

51i

Highogt Alarm

0 Insal Biarri

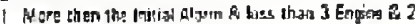

Lador Co. st work.

2 ind hlargh.

3 3it Alstion

4 4h Bizrm

5 in flagm

5. Smulatanoves:

Fignal 7.5

\section{How Exisnguistom}

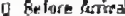

1 Hand Eurequthrs

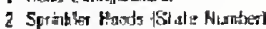

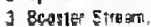

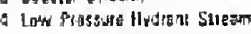

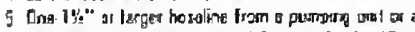

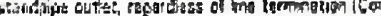

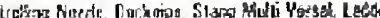

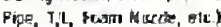

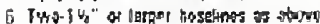

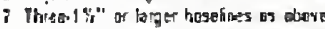

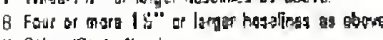

4 Other fitade Howe:

Ifunition Stapa-Termination Stago

I Srolde stega, botare any flomic.

3 Fiome Stapg.

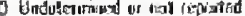

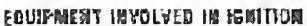

Wuating Syitema

11 Centers hesting urat

12 Hetgr heater

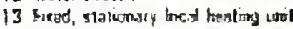

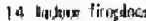

15 Pirlathe kece tastirg unit

18 Corionmay, gas vite tiu

i7 Chimesy contoclor, vam cominactor.

Ther comicelor, van

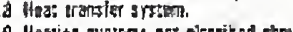

is Heatin systems not clasitis

if Heaking Jrstert.

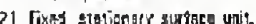

21 Tixas. stamianary surotse

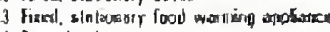

11 Deep tar lire

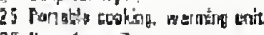

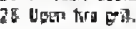

2) Gituast inard, bure

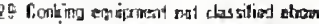

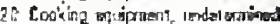

Ais Conditianing, Relrigerstion Equipmatiol

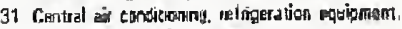

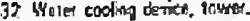

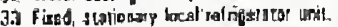

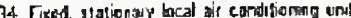

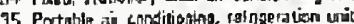

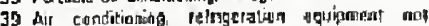
risustied abowe

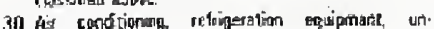
daservings.

Geciticat Ditributjon Eagizmens

41 fixed wring.

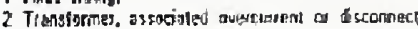
equiprsent

47. Heter, meira trax

44. Poanor switch gos, nuercurreme protection devicas

45. Sinich peceplacts, netioc.

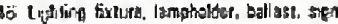

47 Corth plug-

4. I amp, ligh butb.

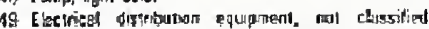
abtryo.

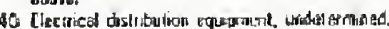

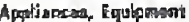

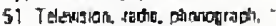

52 Bryer

53 Warhing tortion

4 these exrif paisument

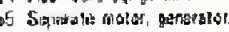

56 Hand tools.

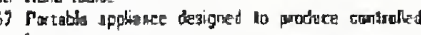
Insti.

58 Portsela sophrase designed not to produrs heat.

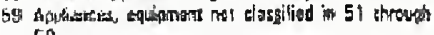

59. Agoronkes, equiginent, undeleanined.

Speciel Equipment

61 Fleringníc घqu'pment

62 Yending mecture, dibiling farintion

$6 \$$ Offie mschins.

64 Bicosesfel pqupmint dence.

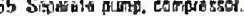

Es Combustion engine.

67 Conters

Eb Printing pres:

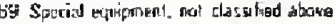

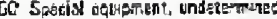

Frocasalog equipman

If Funace, aven, hal

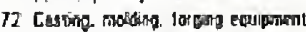

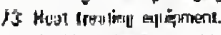

14. Horking shapeng mactive.

75. Coalus mostinis.

7f Panting equiprnent

77 Chemicel process equiprnenl

16 Wosta recuritry enupipment

79 Pracorsing equipmenl, not classifiod vove

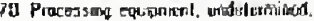

Ssrvied, At aistanamee Equiprpen!

a) heinesstox.

82 Baring. Urake

83 Bastifis, eh orger.

84 Tapoor. yar thertle.

$85 \mathrm{Arc}$.

66 Elerates.

8) Turchas.

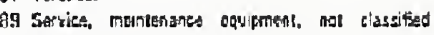
abovit.

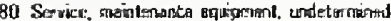

Oithes tititers, Expoesure Flse

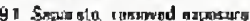

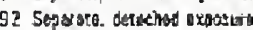

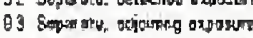

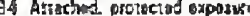

95 Aigathed, unguteclet giressule

35 Vehis:

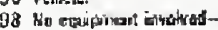

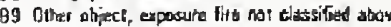

81 Othat objaci, axposune fire.

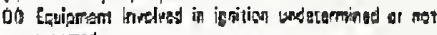
lepunt.

F ORH OF HEAT OF IGAITIOF

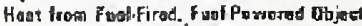

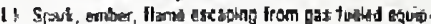
ment

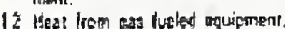

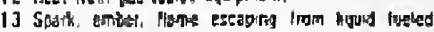
equamsant.

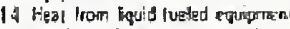

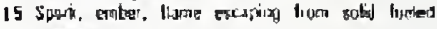
equetritunt.

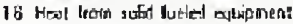

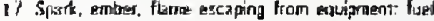
nal hiown

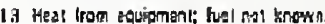

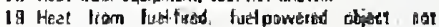
clarsifigd slove.

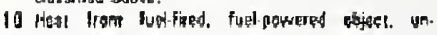
sereminged

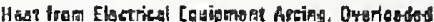

21 Wutur latuserd sharl Evercil aic.

2? Shath nircuit are from mechäncal datrage.

23 Shore rirevit aec from defectivo, worn intula?

2t torispecitiad shon circyic sec.

25 dis fiom fathy suntact, locse sonnertion. biaten eanducior

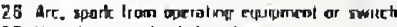

3 Hest from orestasded squipment

zi f Worascent light batast.

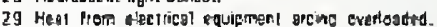
hal classillet above.

20 Heal tran exetrical exutamen! anc ing. overloaded

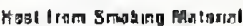

It Cigarente.

32 Cigar.

33 Pine

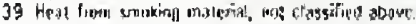

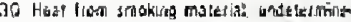

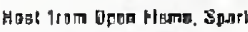

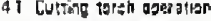

52 Wetrang larch operanan

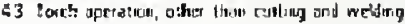

of Corvile, lapur

45 Mtitcis.

tis lightrer.

47 Open fir

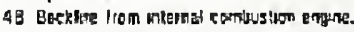

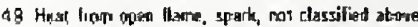

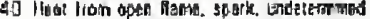

Hleat fram Hot Dbjact

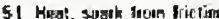

52 Molitan, tor material.

bis Hat simber, bsh.

54 Electric lamp.

55 Rexindte. rewgrmilion.

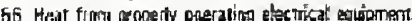

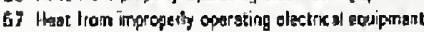

59 Heat from bel ohyed, mat thassfied deavt

50 Hrac thom hor o trect, andalemened.

Mest Iram taptoxive, Firsworhs

G) Esplasis

62 Btasting tชด

63 Fistoratio.

64 Pasar cape porty popts:

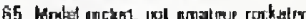

ES Inesiduty dense

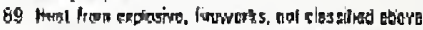

60 thost from axplosive. Heworks, unstelorrimest. 
Hoal tram Haikrat $500 \mathrm{reg}$

11 Suirs that.

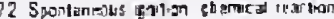

If bightrring dischaly:

is Slatic distharge

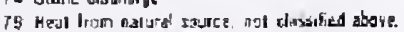

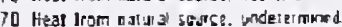

Mast Syrading Irom Anathar Harila fire en posurn]

B1 Heat 15om surec; flame, convecion curtents.

32 Ruilated hal

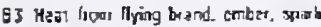

Q4. Conducied beat

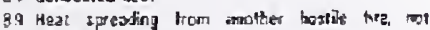
chinglize abeve

80 Heal wiestimg from anothat husile tire, wh dastrmines.

Other form of Hest al I prition

8) Mulipla forins of head of ignition.

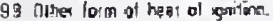

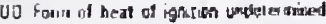

TYPE OF MAILAIRL IG AITED

Gas

11 Nato al gas

15 Lp.grey gas Ito and ail míl

1) Manuiaciuret gas

14 le gas.

15 Anesilitic als

is arstylpis

17 Spasaly ass althes than anesihetic.

19 Cas not elassifued abort.

$10 \mathrm{Cs:}$

Flsprabite, Combustion lag aid

2) Giast A I annabls hasuat

is Class is II ammable kqued

73 ist5ye

24 thass IC ta a unosole linud.

25 Class II camiose thit aquid.

Ci Class IIIA comoustion họud.

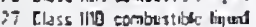

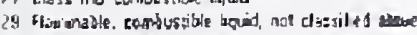

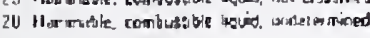

velatile solid. Chomica

j। rat. gresse llood!

i2 rengase imsnleadi.

j] odist

34 Acheswe, resin, 1.3

35 Aevlied bothe vetwest

36 Comsuscibla merel

31 Solut ctrameal Itgesil's topel

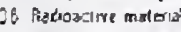

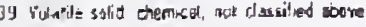

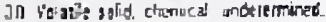

Plastic

d) Polpriselliare.

1. Pobstriten

1) Dolpuint

is Palpararie

45 Paliestu.

46 botiofin

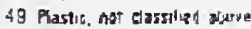

20 P.esris, yrinteminec

Aatural Pratusa

Si Rulee?.

5? [ei.

53 leartel

51 Grons leaves, han seraw

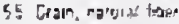

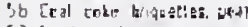

is? Hund arents

58 Truares

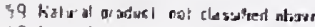

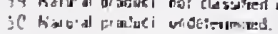

Wood, Paper -

6! Cpewilsy wn nor

C? felled but ursisun ivoed

63 sawn woot.

bi Hoot shavisos

E5 Harchoust, flywand

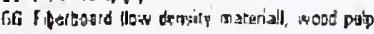

b) Papst, tancessed. theosizt

6a Carillusad.

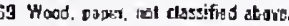

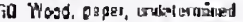

Fabrie Io alita, Fur

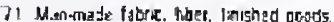

72 Coulons rapan, cotion labrie, fenished giods

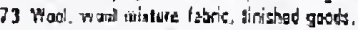

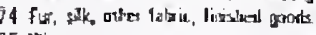

$75 \mathrm{Hig}$.

18 Hentasa hav.

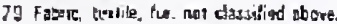

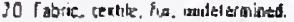

Malerial Cocmpoundad with oil

81 boslem.

B? Cil chlo

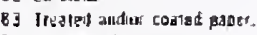

Ca Warerpisal Eanos

B5 Q3y rass.

95) Asphalt thesed ralenal

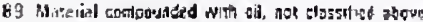

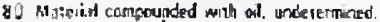

Dera: Tyge ol Material igaingat

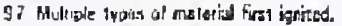

98 Type af mainit nal apalicatso

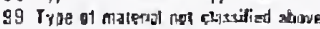

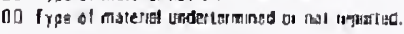

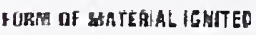

Sirystupol Componont, Finish

11 En:?hos nol ctruesiog. surlaca. Frist

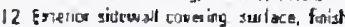

1] Exteriar mis. opxur!tancts.

1: Facr couzing sullace

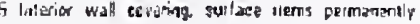
illuses to nall and toot sulast.

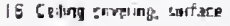

17 sisciura rembor fraroint

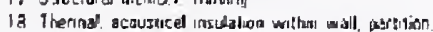
if IImposeiling space

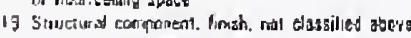

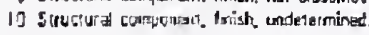

Fursiture

27 Uanousterex soía, chin, artiele seres

22 al arsphalstered than, teract.

i3 Labinsisy

24 lomin base.

25 Apphance hevaring ar tasiog

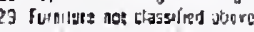

20 furmiters, undetervined.

seit Goods. Wabring Agpare?

3) Masuress pillow

32 bediding, banket ethel, evirfarter.

33 tinen, other thing hiolding

31 Hearing spates nat on a parson.

J5 Fow

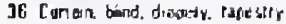

37 ficots not rede se

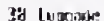

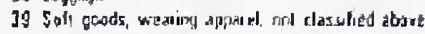

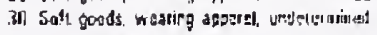

Atomment, Racreationsl Holariel

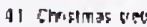

1) Desorsion lar joecial rvent

4) B(x)

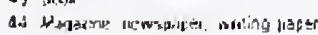

45. Tip arme

16. Aikning. Garoch

47. Iteputh ith

49 Aderament. recieational marenal maz clajsufid

aboves

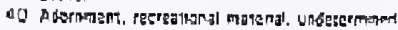

Suppliora. Stacs

51 Bur, bial tim, bin?

52 Bashet. tarrel

53 Psler, skid (not in usp)

54 Mosa. eard tavine ran.

$\$ 5$ Pseliking wrating materiat.

56 Bate secrage.

5.7 But Fiaran.

5.8 Clearinc supplies.

50 supptes, slack rot thas silled ahare

50 Sugpses, slock, undeletrianal

Powar Transtar Equipment. Fuel

6) fleracicat wiar, cabte ircutation.

1i.) Tianslatringr.

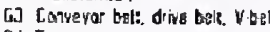

Got Tiré-

65 fual.

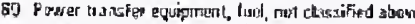

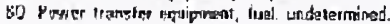

Gonatal Form

TI Agriculiural predust

72 Feace. pole

79 Fertilize:

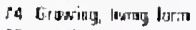

is Kintaily. histi, waste

if Comkerig materiats.

7 Sung.

Spacial form:

(2) Ulust liter. Jun

6) Ppolic.linir. ze explasives

E. Alonizet, maporized lianid

B1 letwos

E5. Fallelizad maiterisl, material stored sa pahel

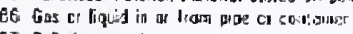

ET Roghers maperat

E2. Achesive.

Qhet rasta of Haterio

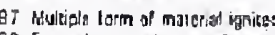

96 form al macerial nol appleable

95 farm al matgrial not classilacel aluove

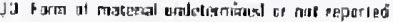

IONITLON FACTOA

Incendiarr

11 kareaclisyy, nat durigg civil disiurbance

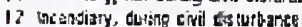

Suppiciacs

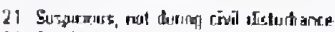

22 Sicmii inus, du in civil disturbance.

Misese of 4001 का Ignirion

31 Astianterith disearded material.

J? Eraming

3.3 :allikg asteep.

34 Inasecuare esaciol of acan live

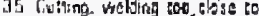

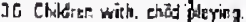

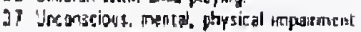

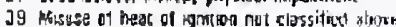

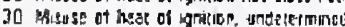

Hisusa of Merarial ignirad

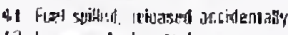

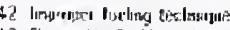

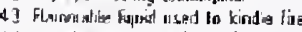

41 wasiang ant, cle aning, refinishing pointro

4.5 Improsas contgirer.

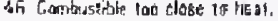

47 impropes stolaga

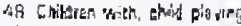

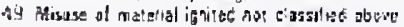

at Mosuse of material ignied. ur Jelesinaral 
Methenizal Failuro. MaHunction

51 Part ladure leak break.

52 Automatic controt falure.

53 Menual control folura.

54 Short citcuit, ground faut.

55 Other electrical fadure.

56 lack of maintenance, worn our

57 Bsetfive.

59 Mechanical failure, malfunction not classified above.

50 Mechanical lajura, malfunction, undetermined.

Dosiga, Construction, Instaliation Deficioncy

610 sion deficiency.

62 Construction deticiency.

63 Installed too close to combustides.

64 Other instafation deficiency.

65 Property too close 10.

69 Desion, construction, instalation deficiency not classified above.

60 Design, construction. instatiation deliciency. undeter mined.

Operational Deficiency

71 Colfision, overturn, knockdown

72 Accident ally turned on. nol turned oft

73 Unattended.

74 Overtoaded.

75 ipomianeaus heating

76 Improper startup. shutdown procedures.

79 Operetional deficiency not clessified above.

20 Operational deficiency. underermined.

Matural Condition

81 High wind.

Q2 Eartiquake.

g3 High water, incluoing floods.

84 Liphining.

89 Natural condition not classfied above.

80 Natural condition, undetermined.

Diher Ignition Factor

91 Animal.

92 Rexinded Irom a previous fira.

99 Other ignition tactor not dassified above.

DO Igntion lactos undetermined or not reported.

Construction Class

D No Building Involved.

1 Firepiool Structure

2 Fire Protected Siructure.

3 Non-fireproof Structure.

4 Woad frame Silucture.

5 Metal Siruature

6 Heary Timber Siructure.

Clessification of Building By Usa-Commarcial

01 Bank.

02 Brewery.

03 Coal Pockzt.

04 Oepartment Siore

05 Electrical Powe Plant.

06 Factory. Mutti Occupancy.

of Factory. Sengie Occupancy.

$0 B$ Feundry.

O9 Freight Depor

10 Garae: Non Siorage.

11 Garage: Stor age

12 Gas Works.

13 Lumber Yad.

14 Motor Vetricle Repair Shop.

15 Office Buiding.

16 DA Selling Stetion.

17 Di Storage Plant.

IB Pier, Wharve. Dock. Bulkheed Building.

19 Reslewant, iner.

20 Shed. Newsstend. Shanty

21 Shipyard, Drydock

22 Stable.

23 Siezm Generating Plam.

24 Store Buiding Taxpaye

25 Warthouse. Stciehouse.

26 Store Building \& Pinate Dwelling

39 Othe Commersial
Clossitication of Building By Ure-Public

40 Airport Building

41 Asylum.

42 Bridpe.

43 Bus Terminat.

44 Church, Symagogue.

$45 \mathrm{O}$ ance Hall, Benquet Hal.

46 Oispensary, Clnic.

47 Fertr Terminal

Governmenl Buldings-(Not otherwise classifeos):

4B City.

49 Interstate.

50 Feder al.

51 . For eign.

52 Stare.

53 Hospita, Infirmary.

54 Nus sing Hame.

55 Ratioad Siation.

56 School: College, Unues sity.

5) School: Private High.

5B School: Public High.

59 School: Pudlic Jr. High.

6D Schoot: Private Elementary.

61 Schood: Public Elenemtary.

62 Schoot. Children's Nurseay.

63 School: Other.

64 Television Studio.

65 Theatre, Leginimate.

66 Theetse, Motion Picture.

67 Transit Sysiem-Station Strueture.

68 Tunnel.

69 Othe Public.

\section{Residentiol}

80 Apartment Hotel "A."

BI Apertment House "A"

B2 Boarding House, Roommg House "B "

B] Convent. Rectory. Monastery, etc.

B4 Damitary-Sctrool, Club, Lodoe.

BS Hotet "B."

B5 Ladging House " $B$ "

B7 Motel.

B8 Privale Dwelling: One Family.

Bg Privete Owelling. Two fomily.

90 Tenemant: New Law "A."

91 Tenement: Old Law "A."

97 Converted Dwelling " $A$."

92 Converted Dweling

Building Stotus

1 Occupied.

2 Pertly Decupied, Good Condition

3 Partly Octupued. Deternor ating.

4 Vacant.

5 Under Oemolition.

6 Under Construction

Oamage he Building of Contenta)

0 None.

11 10 15\%.

216 to $49 \%$

$350 \%$ or Greater

Ares löre Origin-floor

00 Dutside Building

01 ist floor.

9494 th and Higher.

95 Attic.

96 P.oot.

97 Basement

98 Cellar.

99 Sub cellar.

Ares Fire Origin-Roorn or Area

10 Ares Not in Bubling

11 Atii:.

12 Awning

13 Baicony.

14 Besement.
15 Bathroom Totet.

16 Bedroom. Sleeping Aran.

17 Celing.

18 Cellar.

19 Chimney.

20 Classroom lecture Area.

21 Claset.

22 Cocklott.

23 Courn-Exterior.

24 Court-Interior.

25 Dining Room, Dining Area.

26 Ouct-air Conditioning.

27 Duc1-Exhaust.

28 flooring.

29 Furnace Room

30 Halway-Private.

31 Hatway-Public.

32 Incinerator Closet or Room.

33 Kinchen, Cooking Arsa.

34 living foom.

35 Louby.

36 Machinery Room

37 Olfice Area.

38 Operating Laboratory Area.

39 Partition.

40 Parch.

41 Projecion Booth.

42 Recreetion Area

43 Roof.

44 Sales Showroom Display Area.

45 Shaft-Ouct. Pipe.

46 Shat-Dumbwaiter.

47 Shath-Elevator.

48 Shatt-Exterior light

49 Shatt-Interior light

SO Shatt-Yent

51 Shipong Receiving Loading Area.

52 Stage.

53 Steirwey.

54 Storage Room Area.

55 Vacent-Room, Apartment on Aras.

56 Wark Ared. Horkroom.

57 Dither Areas. Not Classified istete ares.

Ares fire Origin-Decupency ClausifieationCommartial

Fecterr:

99 Chemicals.

Clothing:

0) Dresses.

02 Undergament.

03 Other istete irpel.

D4 Ory Cleoning Landry.

05 Electrical Products.

06 Food \& Drink Products

07 furniture.

OB Furs, Fur Goods.

Hats.

Dg Men's.

10 Women's.

11 Leether, Leather Products.

12 Machine Shop Metal Work.

13 Paints.

14 Paper Products.

15 Petroleum Products.

16 Plastics. Rubber.

17 Printing \& Alied industrues.

18 Shoes.

19 Textides

20 Toy of Doll.

21 Woatworking

22 Other Factories Not Classified istane trpes.

Stors:

23 Auto Accessories.

24 Bakery

25 Eutcher. 
4

Aree Fire Origin-Oecupency ClassificatianIcontimued)

Sterr:

26 Candy, Cigzr. Stationery.

2) Clothing

28 Depariment, lage.

29 Department. Smal 15810 .

30 on Cleanes \& Talok.

31 Drug.

32 flectrical Appiances

33 Frui \& Vegerables.

34 Fumture.

35 Grocery. Dary. Delcatessen.

36 Habersashery.

3) Iades Accessories.

38 Land $y$.

39 Paim Hardwaxt.

40 Restaus ant Luncheonetie.

40 Restautar

42 Shoe Repa

42 Shoe Repar.

44 Twern.

45 Dthe Stores Not Classilied istate typel. Garages:

46 Non Storage

$4)$ Storag.

4B Oa Selting Station.

49 Motor Vehicle Repar Sroo.

50 Olfice Bulding

Worehouse:

51 Find.

52 Paper. Aags. Fibre.

53 Other Istate iypel.

54 Fienght Depor

55 Pres.

55 Pres.

5) Limber Yad

58 Shed. Newestand. Shanly. etc.

59 Dither Commercial Bulding Occupanices. Not Classited lstate irpe:.

\section{Residantial}

60 Aparment Hotel, Muliple Owelog "A."

61 Aowiment House. Multiple Oweth "A

62 Boarding House. Rooming House. Wutiple Dwetium " 8 ."

63 Hotel, Multigle Dwething "B "

64 lodging House. Muhaple Owehing 8 .

65 Prvate Dweling.

66 Rectory. Corvent, Monastery. etc

6) Tertement House. Mew Lm. Muthe Owehing "A."

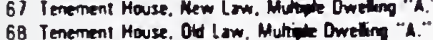

69 Other Residential. Not Classiled tate typel

69 Other

70 Arport.

12 Church.

13 Oance $\mathrm{Hall}$

14 Mosert.

15 Mation Piciure Theatie

$16 \mathrm{NV}$ Tiansit System-Station.

II Passenger Depor.
18 School

19 Theatre.

BD T.Y. Studio.

B1 Dther Public, Not Classified Islate typel.

Menner Extunsion

DO Contined to area of origin.

01 Cocklott.

02 Door or Opening Between fooms.

03 Ftoor.

04 Hall Starmay.

05 Partition.

06 Pipe Recess.

D) Shati. Dumbwaiter.

08 Stalt-Elevator.

O9 Shalt-Ar. Iight. Chute. Duct, etc.

10 Ceiling.

11 Window

12 Orther (stale how)

Number of Dccupancies

011 Decupancy.

022 Occupancies.

9999 or more Octupancies.

Buildings

0 did not saread beyond buiding ol orgin.

1 isincturs of vehicle.

99 or mara buidings or vehicles.

Nore: Form BF $24 A$ must be submitied lor each building of vehicle listed in this coded space.

Smoke Deloctor

0 No detector present.

1 lomiation type, power disconnected of battery removed by occupant.

2 tontation iype, provided earty warning.

3 loniration inpe, laded to operate, battery powered.

4 Ionization type, fated to operate, line voliage powet

5 photoelectiric iyge. power insconnected of battery

rentoved by octupant.

6 Photoefectric irpe. pronded early warning.

7 Photoetectuic type. falled to operate. bettery onwered.

B Photoelectric type. failed to operate. hine voliage Dower.

9 Not possible to determine if detector operated or not.

Classificatian by Trpe fira of Emargeney Transportation fires

B) Shro. Vesset.

88 Motor Yetict

89 Other Transpostation Istate typel

Won Stuuctaral fires

86 AOV LAbandoned'Derefict Motor Vehiclel.

90 Bonfine

91 Brush G:ass.

92 Demolition Wood. Budding Sile.

93 Cump. Land Fill.

94 Rubb:sh-Outside Building
95 Manhole.

96 N Y Trarsit System-Yard. Roadwoy. Tres, etc.

97 Railroad-Y Yord Randwoy. Tres, etc.

98 Tunnel, Bridge.

99 Dthet Non-Structural. Not Classified (state iypel. Emerpency

02 Chimney.

03 Elevator, Escathtor.

O4 Euplosives Escort.

O5 Fust Ad-Assist Peason/sl.

O6 Finst Aid-Resuscitetion.

07 Marine.

OB Precarious Condition-Sings. Trees, etc.

D9 Subway-Railroad.

10 Water Leak.

II Bomb-Unerploded. Scare.

12 Collapse-Cave in.

13 Consion-Vehicular maident.

14 Controlled Fie, Permited.

15 Flood Condtion-Broken Water Main.

16 Incinerator.

17 Leak-Fue ON, Gasoline, atc.

18 Leak-lihum. Gas. Flam. Yapor.

19 Loghtrong.

20 od Burner

21 Person locked in. locked Out.

22 Power-Electrical.

23 Pressura flupture.

24 Refrigersent Leak.

25 Smoke Condition, Ddor. Fumes.

26 Sprinkter.

21 Steam Descharge.

28 Defective Alarm Device lother than Sprinklefl.

29 Smoke Detector.

30 Dither

Pawer for Equipanent

01123 volts A.C.

0224 volts $A C$.

1116 vohs $0 . C$

127.12 volts $0 . C$

15115 volts $A C$.

$2 B 20 B$ volts $A . C$.

30220.230 vals $A C$.

33231.330 volts A C.

34331 on higher vals $A C$

502550 volis $A . C$.

61 Bulane.

62 Coal, Coke. Charcoal. Peat.

63 Fuel OH. No. 1 or No. 2

64 Fuel OA, No. 3 or No. 4.

65 Fuel Od, Mo. 5 or No. 6 .

66 Gasoline.

67 Kerosene.

68 IN gas (stored es tiquidt

69 (P pas is tored as havid).

10 Natur al or itlumnating gase las a gasl.

11 Paper

12 Procane. I

99 Other. 
This page intentionally left blank

G-22 


\section{Attachment G-A.3}

Explanation of Numeric Codes Used on Fire and Emergency Reports -

From June 1, 1987 to present 
M02-1 Hx-511,

14.1 TYPE OF PEORI

core $M$

1 siruclural

2 Tramsponzion 7 tie

3 Mun-Struktirld Tire

4 Emgraghy nosionse

5 Fise fram

6 deodillosal data ver.2449

\section{HOW HE PORTEO}

Codc $\mathrm{Ha}$

10 lelephorie

20 sicet - Manas

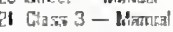

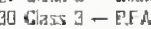

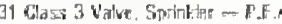

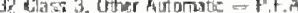

5f Verhat

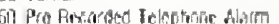

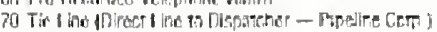

801 Siftel Box-FAS

$910.463-f i s$

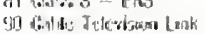

Noto. I. P.f.A stand's dor Privato Finn Ainm

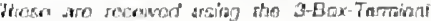

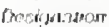

2. It the arim was ercovitered whits

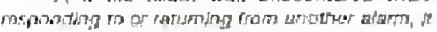

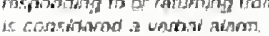

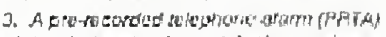

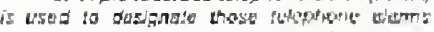

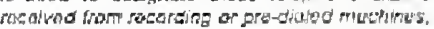

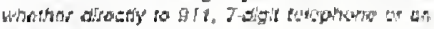
alsom sorvied.

\section{J IMUTHAL ALAIM}

Code $\operatorname{lia}$

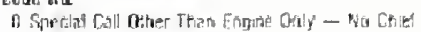

1 fous istrot or Carses 31

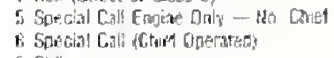

8 Sall

\subsection{HIGHEST RLARM}

CATh $\mathrm{ki}$

(i) |nis|a|

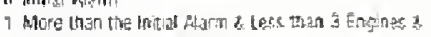

2 calder cas ar work.

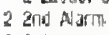

3 ind Aarm

A sth Rarm

5 5 : Al: Alm

8 Sir

7 Signal 7.5

14.5 BUAOUGHS

code ho.

1 Maribatian

2 Grase

3 siaten Isiand

4 Erooidyn

5 Diluens

\subsection{HAZAROOUS MATERIRLS}

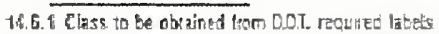
of placaro or fom shipging papers or other documents Cotte Na.

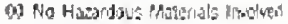

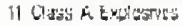

12 Class B Evalosivas

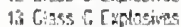

is 8 bisting Ager"s

2) Hannible $\mathrm{G}$ a

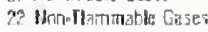

25 hisisan $6.80 \mathrm{~s}$

24 chlants

25 1):
FIRE RECORD CODE LIST

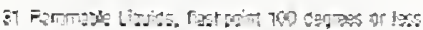

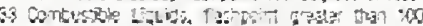
diogrews

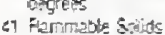

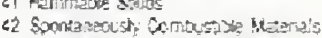

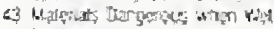

59 cosisirs

S2 orparw: Ferwodes

59 Prow

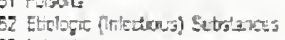

83 irtitatis

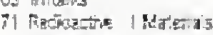

2 Fasiontive fildatis

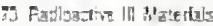

여 Cordistes

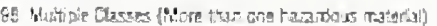

g3 other

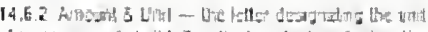
of mists

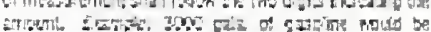

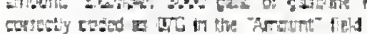

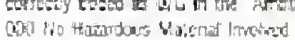

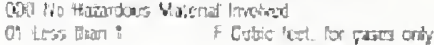

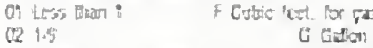

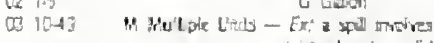

os $100-129$

i iapid and 2 salid

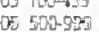

af $1.0095-4990$

$P$ Found

$50002-92 \pi$

c5 10002-49,295

10500029008

0000 knd me?

T4,7 MERTIAIS EJUTPMENT UNDLVEDTIFE OF FUEL USED

code $\mathrm{He}$

i. Herose

2. $2 \mathrm{PO}$.

3. Excutic

4 Visort

5 cos

6 a

7. Histerist

a cigestr.

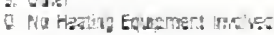

ISB HOU ERTMGUISHED

Cose he

E Eotore atival

7 kizod Exinglests

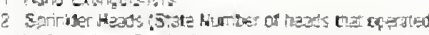

in Che rans semina)

3. Exose Stzan

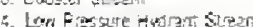

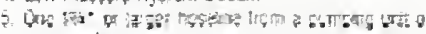

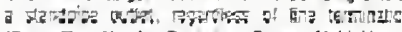

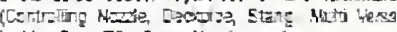

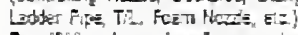

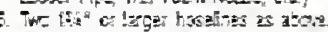

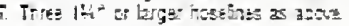

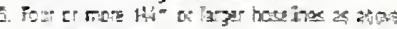

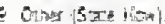

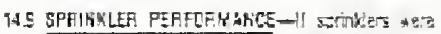

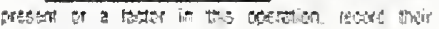
intionest

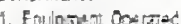

2 Ecuphert in servise tid rot cocate

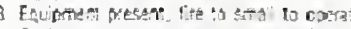

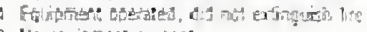

8 Ho equigreteth thesent

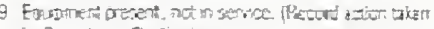

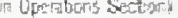

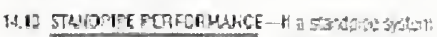

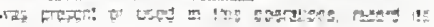

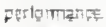

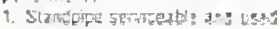

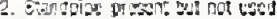

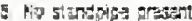

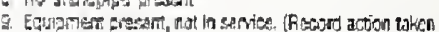
in Cotratons setint

11.11 CCNDITION ON LRTIVAR CODSS

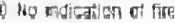

1 Overited

2 s.mol testrog

3 oxen fime

8 Dou an A.Tra

14. T2 EOUIPMERT INOLVEO IN IGNITLON

1. HEMTIG SYSTE

19. Salar panel.

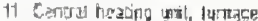

12 Vitic ligd

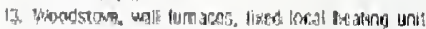

If thror firectito

15. Prowate nestung und

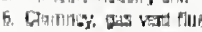

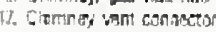

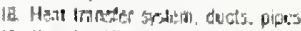

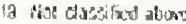

\section{COOKING FQLSTPMENT}

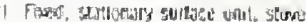

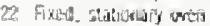

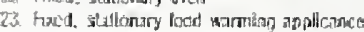

27. Deed fist thens

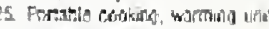

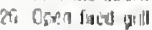

2) Gitate liood. duxt.

2] liot elatiofled above

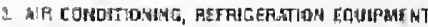

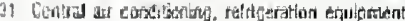

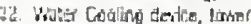

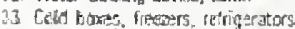

34 Fired. statiatary local als covedtioning urit

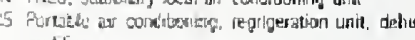
midriase?

39. Nol clastillad athoup

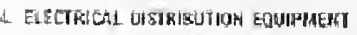

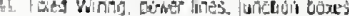

6 .

43. whtow angte tox

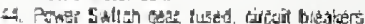

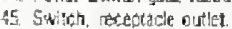

4 Lighting f xture lamp holde ballast. sign

-? Cora plug

4. Lamp Iight both

49. Nat daseifoud shove

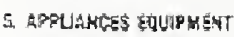

go olstivather:

5: Tyevision, redio. saund ar picture.

52. Govas 40,32

E⿱

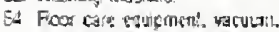

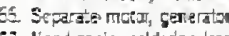

50. Hand zeols, sobchiting leans, dialls

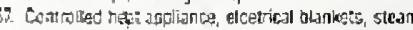
irongy, neal teres.

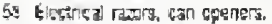

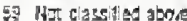

6. SPECIAL EQUTPWEMT

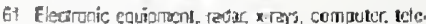
phone transigititar equipment.

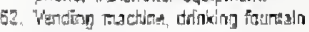

e.s oftice thishiras

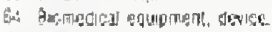

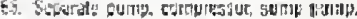

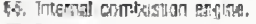

7 SAmesty

14. 97niting oress.

1. Mol classoried atoute 
2

7. PRotressinc EQUIPUEMT

71. Fumace, toner,

72 Casilas, ragling, logging.

73. Haxt trating, quench tank.

4. Wurking. saping, mathins sarks, grinders, staders. हis

75. Cozting machios, asphatl saturating. rexcr speiding machines.

76. Peinlmp. Cippang, spizying.

7. Wasta recovery

79 Not classiticd atoon

\section{Bi. Incinzasint \\ 8. Beyting brike \\ 83. Recurbs: clungen, batient \\ g4. barpot. tar kettic.

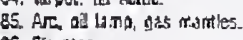 \\ ac. Elemate \\ 87 Lorehes, bunser bumers. \\ \& Nol ctrsibad atsore}

\&. SERICE, MAIWTLUAYCF EQUIPHEXT

9. THER CQJECTS, EXPOSURE FIRE

sü vehicle. exhase spixers, velicetc parts.

48. Ho equipraenl involved.

92. Other objerr, Loposure fire nas claseffied abow.

18.13 FORE PF HEAT DGRTTIOI

1. HEA FROM FUEL-FTRED, FUEL-POWERED OBJECT Tha oflterence benugan stadivision 11 and subdivising 10

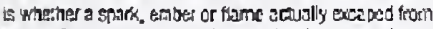

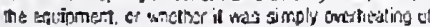

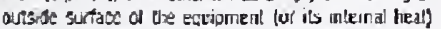
cousing the vorition ol nearty combustibles.

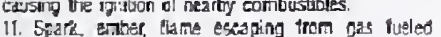
Spark enter

12. Hed: fiom ges fueled cqupment. pilos lights. nama! llamss.

13 5xark. ember farte escaping from lizuid lueled equipmens.

14. Hest Iram liquid fueved equipment, pilot light he

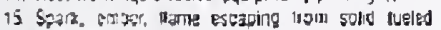
equipment.

12 Hrst fram salat liteden equinmer.

17 Sgark. enter. fiame escroing from equiament. fus not known.

18. Keal from equigment trel sal knumT.

19 hot classitied abss.

2. HEAT FROM ELECTRICAL EQUIPREMT ARCIRG. OVERLOLDEO.

21. Vialel caused shart circuit are

22 Short dixull arc ftan mechanical damage

23 Shor cixuit arc from defective, xorn insulation.

24. Unspecitiox ston ciont arc

25. fre fion lauly chentact, loose ennecion, broken condist $x$.

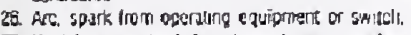

27. Heal fiom overio aded eqaipmem. Whres, motors.

26. Fluarsecent ligtz balian.

"Q Hor claselitios abre

3. KEAT FROH SHOKING MREERIAL

3i. Cigaralte.

32 Cigar.

is Pipe.

ia Net elacsified aborn

4 HEAT FROM OPEH FLAME, SPARK

41. Curtung torch apciation (seprara?ng metals).

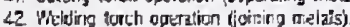

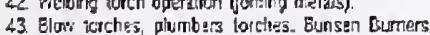
soldering, paim stiping

44 Cankle nazer.

45. Malch

45. Lightes \&f ane typo

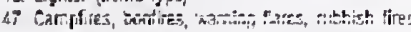

48 Eaxdite from in: ersal sombustios ennine.

43 idor classilied atro.
5. HEAT FRQN HOT OQNEG

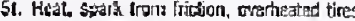

52. Molten metal, hat forming and hot gliss.

53 Hot enther ath.

54 Electic lamp, beght bulbs

5. Fiekändle, reignnilion.

55. Hez from propedy operzing eleatioal,

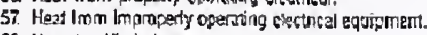

59. Nor classified abons

6. HERT FHOW EXPLOSIYES, RAEWORKS

61. Exploslves, termbs, ammunition

[i. Asating ageni

63. Fickrorics sperterts.

64. Papes cap, pany poppet

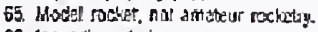

Gi incentiary device

67. Not dassined abure

7. HER FFOM RATHAL SOUACE

71. Sur's hert.

72. Spontaneous Ignition, sitgmical geacton

73 Lightning vos cladry.

74. Sialis dischardo.

7. Not classifad abro

8. HEST SPREADING FROX ANOTHET HOSTILE ITRE [EXPOSUSE)

81. Meat from dired flam-, enfivection cursents.

82. Fadatad hast.

83 Heat from flying brangl, ember, spark.

B. Conducted nedt

$89 \mathrm{Mol}$ clessified abce

2. OTHER FOAM OF HEAT OF TGMIUDN

97. Multiple sorms of theat of igrition.

94 Not classificd atom

\subsection{TYPE OF RATERIAL IGNTED}

1. GAS

11. Hatural gis

12. Li-Cry Gas ILP and air mbx).

13 Manulaelures gas.

14. LRGas.

15. Anestheal gos

ic iserylene

17. Suecirally pas other than anesthetic.

11. Not classified abcie

2. FLAMARARLE. COMBUSTRLF LUUID

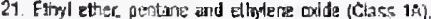

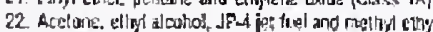
ketong, (Class 1B)

23 Gasoline.

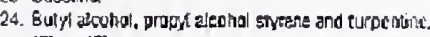
(Class 10).

25. Kercsene fuel ố 1, 2, \&. 5 and Diesel Fuel

26. Na 6 tuct of collonseed oill and crensete oil, (Class IILA).

27. Cooking oil, transtorimer and lubiceing ol, (Class I:13)

28 Nol elásalficd abom.

3. VOLATILE SOLD, EHEMICAL

31. Fat, grease (lood)

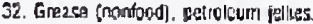

33 Polish, pardetiri, we

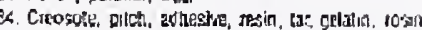
aspiall,

35. Apaliar paikt, vaunish

36. Combusilule metal magnesium ritanium and biconium.

77. Solid chemical, explastues.

39. Hididactivs materis.

39. Ral ciessitied antor

4. PISTTIC

41. กIG IO PI NSTIES

Incilated s:e mats d dasties sych as appliance casps flone tila. decouratur kutchen laminales

42. 816m COAM REASTRE

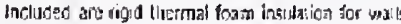
and refruguators.
43. FLEXIBLE PLASIICS

linclused is ctectical wire msulating

4. FLEXIBLE FOAMPLASTICS

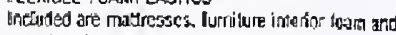
etiper patrs.

45. EIU PIASTICS

Indillded ane miyethytene trash bags. phalsgraphir

flina and costed kall papar

4d plestic nol classified agove

5. NATULALL PRODUICTS

51. Aubber.

52. Conk

53 . Eather

t.4. Gros. leaves, nay and sicen

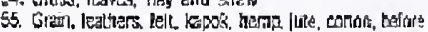
processing.

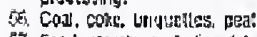

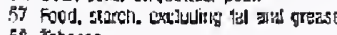

54 Tobacco

55. Not dlascilled abcos-

6. WNCO. PAPEA

61. Growing thod. bre

62. Fellias but unsivil unow

67. Finienes lumber, finshes usod.

61. Whand starings. sandust excelsion

65. Hardboard, plyougot.

66. Fiberboard, wood pulp press board.

67. Paper, unteated, uncoated

E. Cardboard.

69. No? dasiilied aborre.

T. FARRIC, TDTILE, FUS

71. Kham-madr tabric, fiber, funinher rooks

72. Collom, ragen, cotton tabrî̀.

73. wol, hopl inixiste. tabrile

74. Eur, silk, other labric.

75 Wít

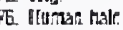

79. Nat chasstlies above.

8. MATEPIRL COY POUNOED VITH OIL

81. Linoteum.

Q2. oit thom

63 Treated entsot cuated paper, wax paper

Q4. viatertor of canvas

85. Q4) दags:

\&. Asphat trisaies material.

gg. Na: ciassified abriva,

9. OTHEA IYPE OF MAIEGIAL IGNTED

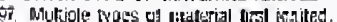

59. ald cizestica abion.

14.15 FORH OF MATEAIAL TORIEED

i. STRUGTURAL COMPONENT, FINISH

11. Exteriar roa! cosering, santace. finish

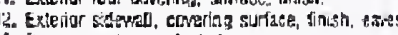

13. twors, purches and plattorms.

14. Tile, Gerpet, rug thooring and stairs.

15. interig wall coweting.

16. Ceiling comerrerg surtec

17. Siriclural meriber, sracning.

18. Thermal. acoustical insulation, villicia wall or ce.ing

19. Riot chassificd abtope.

2. FUFHITLAE

21. Uphobsted sofa, chair, vetirle sear

22. Nonuphalstered chalr, banch.

23. Cabinetry. fling cahlinets, piasos, dressers, desks tables aid bockcases.

24. Iróning boe id.

25. Aparhónce housing at casing

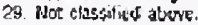

3. SOFT GOOOS, WEARING APPAREL

31. Mattees, pillow

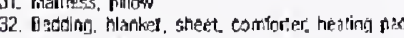

33. Linè, towels, tatierioths

34. Wealing apparel ant on a parses?

35. Wearing apparel on a pesan.

36. Cint dir. ulau. didpery. lapesiry.

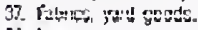

3.. L.tggaye-

39 Hot classifind abur 
4. ACOBMMEN, AECAEMTOHAL MATERTLL

4. Crotisomas tree

43. Oegoraten far spetial wan:

40. Box.

44. Magasine, remspzpes, witing paper. lucs.

45. Toy. game.

4t kesring. canopy

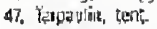

49. Nat casilia abom

5 SUPPUES, STOQR

5) bax, cation, hat

32. Bagkct, baste

53. Fallet, shd.

5. Fipe, cord, Mine, yark.

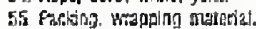

56 thle जoroge

57. Bulk sturage-

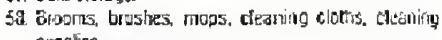
supplies

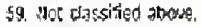

6. POHEA TRAHSFEN ECUIPHENT, HUEL

61. Fiontrical wire, cable lissulation.

62. Tronstoment.

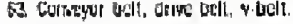

64. Tire.

is ?

bis Nor classified aboue.

7. GENERAL POHR

7h. priguttual product.

72 fence pole

31 Fetilizar

7h. Forstis, trush and grass.

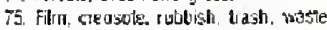

Fis Cooking materizls.

if sign

c. SPECIAL FORP

81. Cusl. itber. In t. Samulust

Q2. Pirotechnirs explasives

83 Acornined, waporized lingió

ช. Crips.

85. Mil!ngal stersd on pallot.

65. Pecelerants.

B. Rollet maten?

88 Nhesire

9. OT HEF FU HM OF MATEKIAL

G. Pulliple form al makrial igrile

99. Hot dassilied above.

14.85 IGUMOHOH FACTOA (CALSE)

1. IHGENGIARY

11. |xcendiary

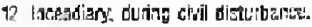

2. SUSPICIDUS

21. 5 เsตic̆ous

22. Suspitious duriag elvil disturtance

1 MISUSE OF HEAT OF IGNTINON

31. Abantioned, dscaded materizl, cigarete, et

7y. Thasing

77. Falling aslege

24. lnesiauda cotiol of ogen lik.

95. Cuting, walding ton elose ro

35 chisten wioh matches. lighe? e.c.

37. Unconscious. mertal. phisical impaimnent

5a lut dascellied abcre

4. MISUEE OF MATEFIAI IT:NITED

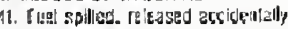

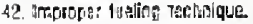

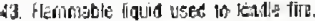

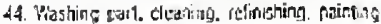

15 Improper ocneaine

4a Combusrabes ioo close in torat

47. impruger slurage.

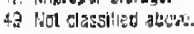

5 MECKLANICAL TAILAE, MALFUTETION

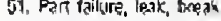

52. Antamitic comid falure.

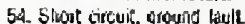

55. Other electrical tailun

56. Lack of maintenasce, wora out. talure is cleent

57. Bacikfice

59. Na: classifies ahme.

6. DESICW, CONSTIUCTION, NETALATION DEFICIENCY

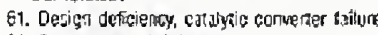

82. Corsiruetsal dothionsty

63. INStalled too elose io cotnbusthes

6t. Qther In atallation defitisncy.

65. Pinperty lao clase in tacluded asp exposura fipas.

89. Not classified atrove

7. OPERATIONAL DEFICIFNCY

71. Callision, omenturn, krockolowa.

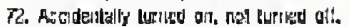

73. Unattended.

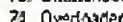

75. Sprontanieous heatinn

7b. Ilisurupes starlup. shuid own procedures.

79. Ne1 classifed abore,

1. Naturar conbrion

81. kigh พาน

82. Eartisuzuka,

23. Iligh waler ineluding Hoods.

E4. I. inthing]

Eai Nol classilied abone

9. OTHER IFHIJOM GARTER

97. Minilus

92. Hekinded liem a provrous tire

99. Nol classified abure

co. Ho Tire

\subsection{JUVENLE IRYOLVED IN IGATIION}

0. Jv verile Nol Indwod in lgnitian or No intormatian that

a Jusnile Mas IRTQlyes

1. Juvenile Irmolves in Igrtion.

I4 18 constaยนTION cLres

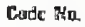

0. Na building Inolved.

1. Freptont sindate

? Fire Proteged Strueture

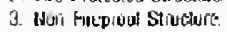

4. Wood Irame Stunture.

5. Mptal Struceur

6. Heany Timber Structure.

14.12 ELASSIFICATION OF EUILDING BT USE

COMHCNCLAL

92. Dank

T23 Riewery

b95 Coal Sistage.

5itit Depanment Stare.

615 Electrical Ponver Plant.

zod Faclory: Mutiti- Oxcopancy.

Tos Fectory: Sirglit orcupancy

33 iogridiy.

Bys Freight Bepot,

Baz Carge: Nom-Starage

899 Garage: Slonge.

7 Gas Whars. Natusal Ges Piant

851 wimber Yar.

573 Ho:de Vehicle Repio shos. Paist sime

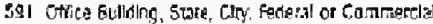

s71 on Soling S:atich.

Ast Oil Storage Piant

AGg Pier, Whar, Dack, Eulthead Euilding

lit Restzuan: Diner

\$as snepd, tworstand, snanty.

81 siapyas, Dheluok,

tis Slabis

S14 Staam Genaraling Plant.
539 Siorcbuiding. Taxplyer.

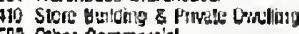

50 other Cosmmerial.

PUbue

171 Airporl Building.

351 Asylust.

Q21 Eridge.

13 Pus Terminal.

121 Dince Halt Banguet

334 Dispersary. Cunic

iT) Ferry Ternom.

35! Hasplial, Infirman

319 Nursing Horne.

Iit Railioat Stztion, Street Level.

Tis Railosus slation. Berox Grade

is Bailrsat Station, Mhone Gade

241 St네uyl; Collexs, URiversity

215 Sthool: High Sthool

244 Schuod: Junior Hich

213 School: Elementar

211 School: Thildren's Rutsery

270 Schuot: Other.

185 Tolevision Studia.

163 Thestre Mogitimiatc.

nio Transil Systen-Stakina Struelute.

22 Tunnel.

119 otrer Public

AFSIDERTIA

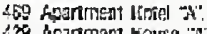

439 doarding House, Rnoming House " $\mathrm{B}$ "

465 Comnat, Restory. Munazlery. Ete

451 Dormitoñ-Schod. Cusb. Larpe.

4 प Kescl " $\mathrm{B}$ ".

430 Lofinian House $x^{\prime \prime}$

40 Mutel.

419 Privake trumelinnt: One Family

414 Pervate Drrelling: Tuso Fanuly

420 Tenament; New Lär "W?

ç3 tenkTeth: Dis Law "

490 Uther Pesidential.

SPECAAL PROPERTIES

$9 R$ Airpor Rumber

gat Cemcley.

991 Construction stre

932 Owmp. Land fel.

93) Opsn La al, Fiald

Serking Area. Lo

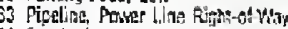

tot? Public Siresh.

852 Railmad Svitehing Yard, marshalliag yerc

936 Vetanit Lats.

gjg Ou;dnes Property Not Classifion.

\section{0 gulLojng sTatus CODE}

Code Deswiguan

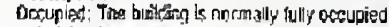
wi is intend ted to be fully occupied. A lev vacant ereas, winten ate ientinte, may exist

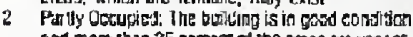
and mone than 75 percert of the reas are vecant.

3 Party oneupied, Deterioratiog! The tulding has same vacant a aess and thuse ale expacted to Jemain vecant until demation or alieration heca lse

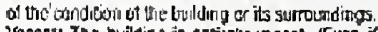

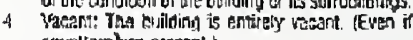
spvaltersitare presenti.)

Under Demagtion: The building is in the process al being tom down.

6 Uader Comstruction: The building is under constiugann and does not hime any dccupants.

7 Unotgl Cunsusution: the building is astially ofcupies. whether under a temporary centificate of

Note: The status cose applics to ale buitsing. not the fire ara. Theretore, cod's.s 1,2 and $3 \mathrm{mzy}$ be

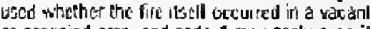
or cocugizd area. and exde 1 ma; apply even jl

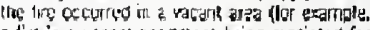

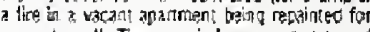
e nex teitanlh. The acsupied ar vacans status a the fine arre is new reconthofion the "Atca di Origin'

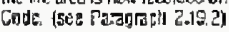


4

4. ช COMPLEX

11. PUELLC RECRCENON COUPLEX

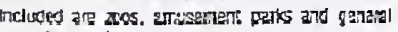
recreton parto.

12. STADNUM, EXHEITDN HUL CONGIEX

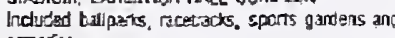
amores

14 GUB CONREER

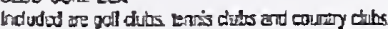

20 EOUCATONAL CDMFL EX

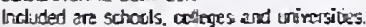

33. MEDICAL CARS CDMPLEX

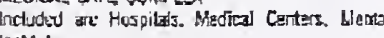
insativitons

34. PFI50N COMFLEX

4a SUSMESS WIH AESIDENTLL COMPLD Induded are apartimeats ares gores

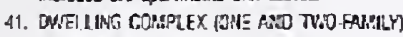

42. APARTMEST CUMPLEX

44. HUTL COMPLEX

theludert are moeets. ixrs and hodges

47. MORLE MDNE Fi.HK CCHPLDX

5a. SHOPPNG CAMPILE

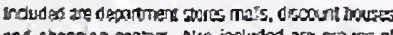
and shopping centers. Niso included are grosps of

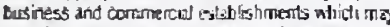
ennain thesters and otty plares of assimbly

3]. OFFICE CONPLEX Intived ate nan-milis?' govemmens otfire complees.

61. POWER PROOUCTIDR LOMPLEX

63. MLTRAT FESERMATION DËTNSE COMBAMY

65. FAPUN DOMAPIEX

70. INOUSTPAL PLAMT. MANUAACTURNG COMFLEX

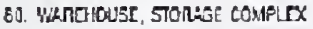

פ1. COMSTRUCTION COHPLEZ

Indudod are arcoitivi operations.

Q3 CAMPSTL COMPLCX

94 WETERFRONT COMPLEX incured are naniss

95. RALPOAD TREYSPOAT COLPLEX

ST ROND CONFLEX

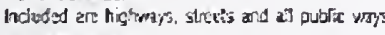

97. लARTOI CAMRE

Q2. No COMPLEX

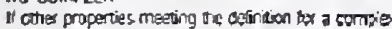

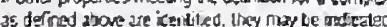
by Coniolex Coote sin.

AFEA FIRE ORIGIS

3422 FLOPR CODE WO.

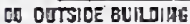

01 1st Ficor

is geth and Higher

85 Aríc

8. Aric

97 Baserem

98 Cerla

99 Sub.Cille:

H.23 AाE FIPE ONICR -

OCTUPAKCY CUSBIFtcartoH

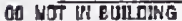

COMrTRCARL

99 Fattorr.

99 Oresses

Q2 Undernaincerd

03 Oitrer (stale type)

We Dry Clezing Leszery

to5 Dectral Products

of rood and Brink Forcures ar Fuminus

ad Furs. Fus Goods

og Lats flats

10 womeris Mats

1 Lealher, Leather Procinds

12 Machone Shop Motal Yook

13 Pairis

5. Petrolesm Products

Plastins, Rubuer

17 Prming ás Alied Industrics

18 stwes

19 Texties

20 Ifor a Dal

21 wpovmonkng

22 Other fectures nod classilied (sath sype)

Store:

201 Aulo Accessonies

25 Gakkry

26 Condy. Gesar. Sistionary

7 Cathis

36 neparmen, brge

29 Depirtinest. small $\{5$ \& 1 it]

30 Dry Cleasar \& Taio

31 Drug

32 Ele arrasl Applitante

33 Fivis and vegeras:

3.4 Fumblere

35 Groosry. Odiry. Delicatessan

35) Histerdisticty

37. Lacier ducessones

38 Lanatiry

39 Fins, Hanthase

40 Restiurant Luncheonatat

41 Sho:

42 Snoc Repir:

43 Supcrmarlal

41. Torem

45 oiner stores nal chasilad (state tha)

Garass:

4i Non sara

Stivane

19. Dil selling Sutation

49 Motar Gehicle Repair Shop

Otrice Guter

Waretouse:

51 国空

52 Pyer, Rags, Fibre

Si3 cluer (state type)

5. Fingitil Dapo:

55 Pict

56 Singyars

3) Lumber yerd

58 Shed. Niwstant, Sharty, atc

9 oher Cormmeral buld no Cocupancies, not dassified (STIT) thPe)

Resivoritlal:

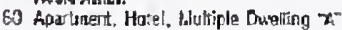

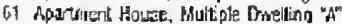

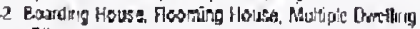

63 Hots. Mulliole Dimeliang "B"

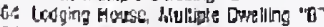

65 PTivate Diwelling

65 Rectory. Gonvent, Monastery. Ekc

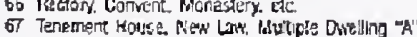

68 Tenpment Hous Ond Low the

6 other Residontiat, no' classoried (state type)

Publis:

To aspert

71 Cabarel. Banquel Hall

72 Churct:

73 Onace kis

74 Hespita!

75 Marion Piture Theato.

T6 N.Y Transit SYyatem Station

77 Passenger Depor

78 sthool

$7 y$ Iliedure

80 เV. Stura

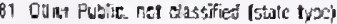

74.24 AREA OF FHE ORTCII

(2. MEAHS OF ECAESS

Q7. Hellyny. corricor, nath

12. Exterige staipwas

O4. Escadetic

05. Lobly. extrance way

Q. liot classirind above

1. ASSEMRELY ARES

11. Fiand seats (100 or more persons)

12. Willoul tixed seats (IDO ne more cersons)

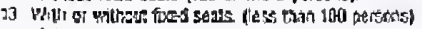

14. Living room, temily trom, trange and

45 Seles showrmom arez.

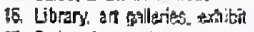

17. Switrining ares

19. Nor classified abose

2. FUMCTLLU AREAS

21. Pedrams, petient roons, celli lockups.

22. Wards, domitaries, barack

21 Dining ares, lurechroom, cafteria

24. Kitchen conking anos duskroum.

76 Lasondry area

28 Healih cluts: Mrssage partars, taroer, beavis:

3. FUM ARENS (cortititurd)

31. Laboratiory

32. Pristing ar phosingraphic raom.

91. First aid, treztment racm

34. Operatisin rom

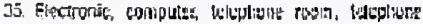
booth.

35. Pertormance. stage area, indocr spontz

37. Projection posial, stage lighl.

sa process, natpuluturing orez

39 Nol classified atwere.

4. STORAGE AFEAS

41. Tank, bin, arocict storspe room

42. Closet

4]. Supply rost

44. Rerords storge resm. weylt

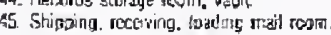

at Tresh or rubrish curlaner, compactor

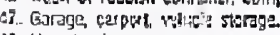

49 No: ctessiticd abor

S SERYIGE FECJUTIES

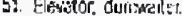

52. Electrical, glun'sing. sentilation shat

53 Light stall.

54. Loundiry or mail chere.

55. DuGl

S6. Display Window

57. Chimney, flue, stampipe

58. Converor.

52. Nor elassified abrow

\&. SERHGE, EDUIPUEHT AREA

Ei. Hachiney mom

62. Heating equipnent, water hezter ares

61 Stratthoess arca, transiarmer vault.

64, inciserator roon ates.

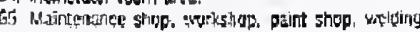
shop.

66. Test corit.

6r. Enchesurg with gressunzed ai:

68 Enciosure with enriched exygan amaghore

El. Not classified abruz.

7. STRUGTURKL AREKS MOH-FUACTIONAL

71. Craw space, cella! substructural a:ez

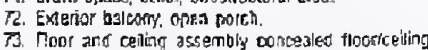
space

74. Ron and teating assembly: concrelted rooticeling space

if Wall assenibly. cunceated vall spare

Pa. Exterur wall suldace.

77. Exteriver wal surface

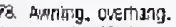

ॠ. Net clascified atris 
6. TRANSPORTATIOH, VEHICLE AREAS

81. Passenger area.

82. Trunk, load carrying area.

83 Engine, running gear, wheel area.

84. Fuel tank, fuel kine.

85. Operating, control area. cab, cockpit.

86. Exterior exposed suriace.

89. Not classiffed above

9. OTHEA AREAS OF ORIGIH

91. On or near railroad night of way. embankmemt.

92. On or near highway, public way. street.

93. Terrace, patio, courtyard.

94. Lawn, field, cpen area, vacant lot.

95. Wildand area, woods.

97. Multiple location.

98. Vacant room, apartment or area.

99. Not classified above

14.25 MANNER OF EXTENSION

Code $\mathrm{Ha}$

Do contines to area of ongin.

of Cocklót.

02 Door or opening between rooms.

03 Floor.

O4 Hall, Stainary.

05 Partition

06 Pipe Recess.

of Shat-dumbwaiter

Os Shath-Elevator.

Do Shath-air, Light, Chute. Duct, eic.

10 Ceiling.

12 Other (state type)

14.26 NUMBER DF OCCUPANCIES

\begin{tabular}{ll}
\cline { 2 - 2 } Codes & Oescription \\
01 & 1 occupancy \\
02 & 2 occupancies \\
$:$ & $\vdots$ \\
99 & 99 or more occupancies
\end{tabular}

14.27 BUILDINGS

\section{Code: 0 to 9}

0 - did not spread beyond building of origin

$1-1$ structure or vehicies

-

9-9 or more buildings or vehicies

MOTE: Form BF-24A must be submuted for each building or vehicle listed in this codet space.

\subsection{8 damace cooes}

14.281 Percentage Codes

O No appreciable damage

1 From 1 through $15 \%$

2 From is throun $49 \%$

$350 \%$ or çreater

14.28.2 Extent cf Damage Codes: To be used in the Damage Category Emes "Flame Smoke and Water".

1. Confinet to object or origin.

2. Confined to part of rom or area of oricin.

3. Contined to room of origin.

4. Confined to fire-reted compartment of origin.

5. Confined to floor of origin

6. Confined to structure of origin.

7. Extended beyond the structure of origin.

9. No damage of this type

14.29 SMOKE ANO HEAT OETECTOR CODES -

14.29.1 Present

1 Present

0 Not Present
14.29.2 Type

1. Smoke

2. Heat

14.293 Power Source

1. Battery

2. AC

14.294 Pertormance

1. In room of fire; operated

2. Not in room of tire: operated

3 In room ol fire: did not operate

4. Not in room of fire: did not operate

5. In room; fire too small to operate

6. Oid not operate; power source remicved

9. Not ctassified

14.30 POWER FOR EOUIPMENT

or 1 -23 volts $A . C$

0224 volts $A . C$.

1116 volts $O C$.

127.12 volts $D . C$

15115 volts AC.

28208 volis $A . C$

$30220-230$ volts A.C

$33231-330$ volts A.C

34331 or higher voles A.C.

50 25-50 volts A.

61 Butane

62 Coal, Coke. Crarcoal, Peat

63 Fuel Oil, No. 1 or No. 2

64 Fuel Oil, No. 3 or No. 4

65 Fuet Oil, No. 5 or No. 6

66 Gasoline

67 Kerosene

68 LN gas (stored as havid)

69 LP gas (stored as liquit)

to Natural or llsuminating gas (as a gas)

71 Paper

72 Propan

Othe

14.31 CODE FOR TYPE OF ACTION TAKEN

1. Extinguishment

2. Rescue

3. Imestigation

4. Remove liaard

5. Standby

6. Salvace

7. First Aid

9. Cancelled Enroute

14.32 CLASSIFICATION BY TYPE FIAE OR EMERGENCY TRANSPORTATION

Code Nie

67 Sinip. Vessel

68 Motor Venicle

89 Other Transportation (state type)

\section{NON-STRUCTURUL FRES}

Code $\mathrm{Ke}$

84 Explosion, no after fire

85 Outsite Soill eak with Fin

86 ADV IAbandoned/Deretict Motor Vehicie)

90 Bonfire

91 Brush, Grass

92 Demolition Wood, Building Site

93 Dump. Land Fil

94 Rubbish-Outside Building

95 Manhole

O5 N.Y. Transit System-Yard, Roadway. Ties, etc.

97 Railroad Yard. Roadway. Ties, etc.

98 Tunnet, Bridge

99 Other Non-Structural, not classified (state type)

EMERGENCY

Code $\mathrm{Ne}$

03 Elevator. Escalator

04 Explasives Escort

as First Ajd-Assist Person(s)
D First Aid-Resuscitation

08 Precarious Condition-Signs, Trees, etc.

os Subway, Rauiroad

10 Water leak

iI Bomb-Unexploded, Scare

12 Collapse-Cave in

13 Collision-Vehicular incident

14 Controlled Fire Permitted

15 Flood Condition-Broken Water Main

16 Incinerator

17 Leak-fuel Oit Gasoline etc

18 Leak-filuminating Gas, Flammable Vapor

19 Lightning

20 Oil Burner

21 Person Locked In. Locked Qut

22 Power Electica

23 Pressure Rupture

24 Reirigerant Leak

25 Smoke Condition, Odor. Fumes

26 Sprinder - Leak, Water Discharge Damaged Head, ete

27 Steam Discharge

22. Defective Alasm Device (other then SprinkJer)

29 Sinoke Detector

30 Defective Alarm (SprinkJer)-Surge. Vhork on System,

etc.

\subsection{MDQILE PROPEATY TYPE CODES}

12 Bus

13 Motorcycle, Snowmobite

14 Motoriome

15 Travel Traler

17 Motile Home

20 Freight, Road transport

30 Rail Transport

40 Water Transport

50 Air Transport

60 Heavy Equipment

To Special Vehicles. Containers

99 Other Mobile Property Types 


\section{Attachment G-A.4}

\section{Significant fire incidents occurring in WTC 1 (12)}

\begin{tabular}{|c|c|c|c|c|c|c|}
\hline $\begin{array}{l}\text { Significant } \\
\text { Fire }\end{array}$ & $\begin{array}{l}\text { Incident } \\
\text { Date }\end{array}$ & Fire Location & $\begin{array}{c}\text { \# Sprinklers } \\
\text { Activated }\end{array}$ & $\begin{array}{l}\text { \# Standpipes } \\
\text { Activated }\end{array}$ & Cause of Fire & $\begin{array}{l}\text { Material } \\
\text { Ignited }\end{array}$ \\
\hline 1 & 9/9/77 & $\begin{array}{c}\text { B-6 level storage } \\
\text { room }\end{array}$ & 2 & & None listed & Not listed \\
\hline 2 & $9 / 23 / 77$ & $\begin{array}{l}\text { Dumpster on B-4 } \\
\text { level }\end{array}$ & 2 & & $\begin{array}{c}\text { Not } \\
\text { classified }\end{array}$ & Trash/waste \\
\hline 3 & $10 / 16 / 81$ & 19th floor office area & - & 2 & $\begin{array}{l}\text { Discarded } \\
\text { material }\end{array}$ & Furniture \\
\hline 4 & $12 / 23 / 83$ & $\begin{array}{c}2 \text { dumpsters on B-4 } \\
\text { level }\end{array}$ & 2 & 1 & Suspicious & Trash/waste \\
\hline 5 & $1 / 27 / 85$ & $\begin{array}{l}\text { Office space on } \\
\text { mezzanine level } \\
\text { (Floor } 2)\end{array}$ & 2 & & 1ncendiary & Trash/waste \\
\hline 6 & $9 / 10 / 85$ & $\begin{array}{c}\text { Garbage dumpster in } \\
\text { service elevator } \\
\text { lobby on floor } 43\end{array}$ & 2 & 1 & Suspicious & Trash/waste \\
\hline 7 & $11 / 1 / 85$ & $\begin{array}{c}\text { Storage closet on B-4 } \\
\text { level }\end{array}$ & 3 & 1 & Suspicious & Supplies/stock \\
\hline 8 & $6 / 7 / 86$ & $\begin{array}{l}\text { Dumpster fire on } \\
\text { floor } 106, \text { compactor } \\
\text { room on floor } 107\end{array}$ & 2 & 1 & None listed & Trash/waste \\
\hline 9 & $9 / 30 / 91$ & Office on B-4 level & $\geq 1$ & 2 & $\begin{array}{c}\text { Discarded } \\
\text { material }\end{array}$ & Trash/waste \\
\hline 10 & $11 / 19 / 91$ & $\begin{array}{l}\text { Electrical closet on } \\
\text { floor } 93\end{array}$ & 0 & 2 & Short circuit & $\begin{array}{c}\text { Electrical wire } \\
\text { or cable } \\
\text { insulation }\end{array}$ \\
\hline 11 & $7 / 23 / 92$ & $\begin{array}{c}\text { Level B-5 at the } \\
\text { power distribution } \\
\text { panel }\end{array}$ & 0 & 2 & $\begin{array}{l}\text { Electrical } \\
\text { failure }\end{array}$ & $\begin{array}{c}\text { Electrical wire } \\
\text { or cable } \\
\text { insulation }\end{array}$ \\
\hline 12 & $11 / 10 / 99$ & $\begin{array}{l}\text { Computer room on } \\
\text { floor } 104\end{array}$ & 3 & $\geq 1$ & None listed & $\begin{array}{l}\text { Plastics, } \\
\text { electronic } \\
\text { equip }\end{array}$ \\
\hline
\end{tabular}


KCTUKI- 2IKULIUKAL RIRE

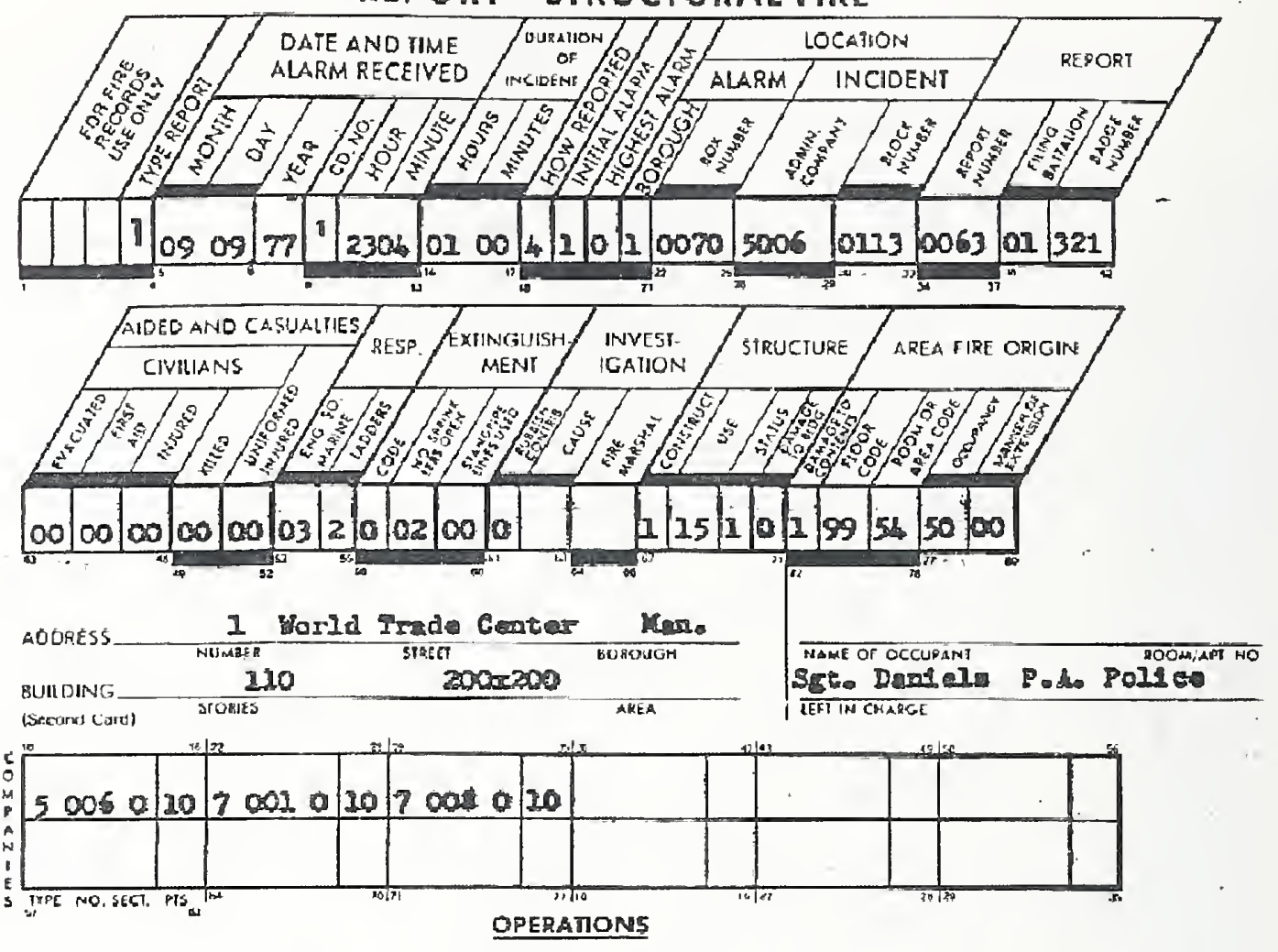

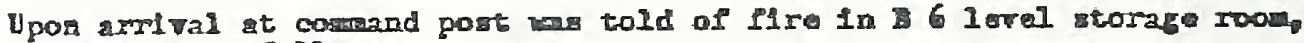
operations as follow.

Ladder 1 made nocessary Intatagation, located Mro, ranted, overhanlad.

Ladder choeked Sor axtenston. vented overhmuled.

Engline strotched 1100 Iros ataodplpe and atood farts

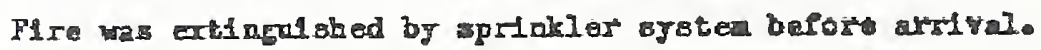

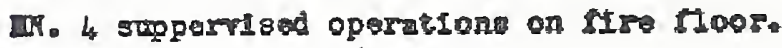

Fire patrole 2 on tho acene.

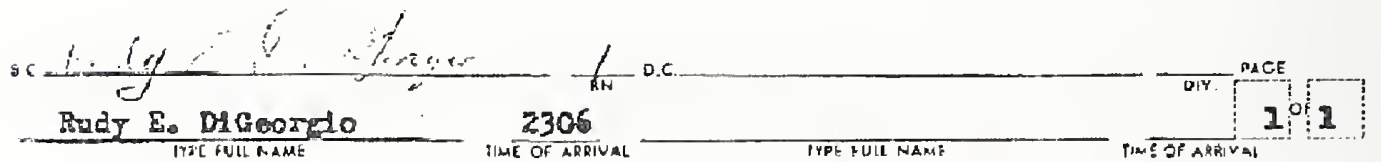




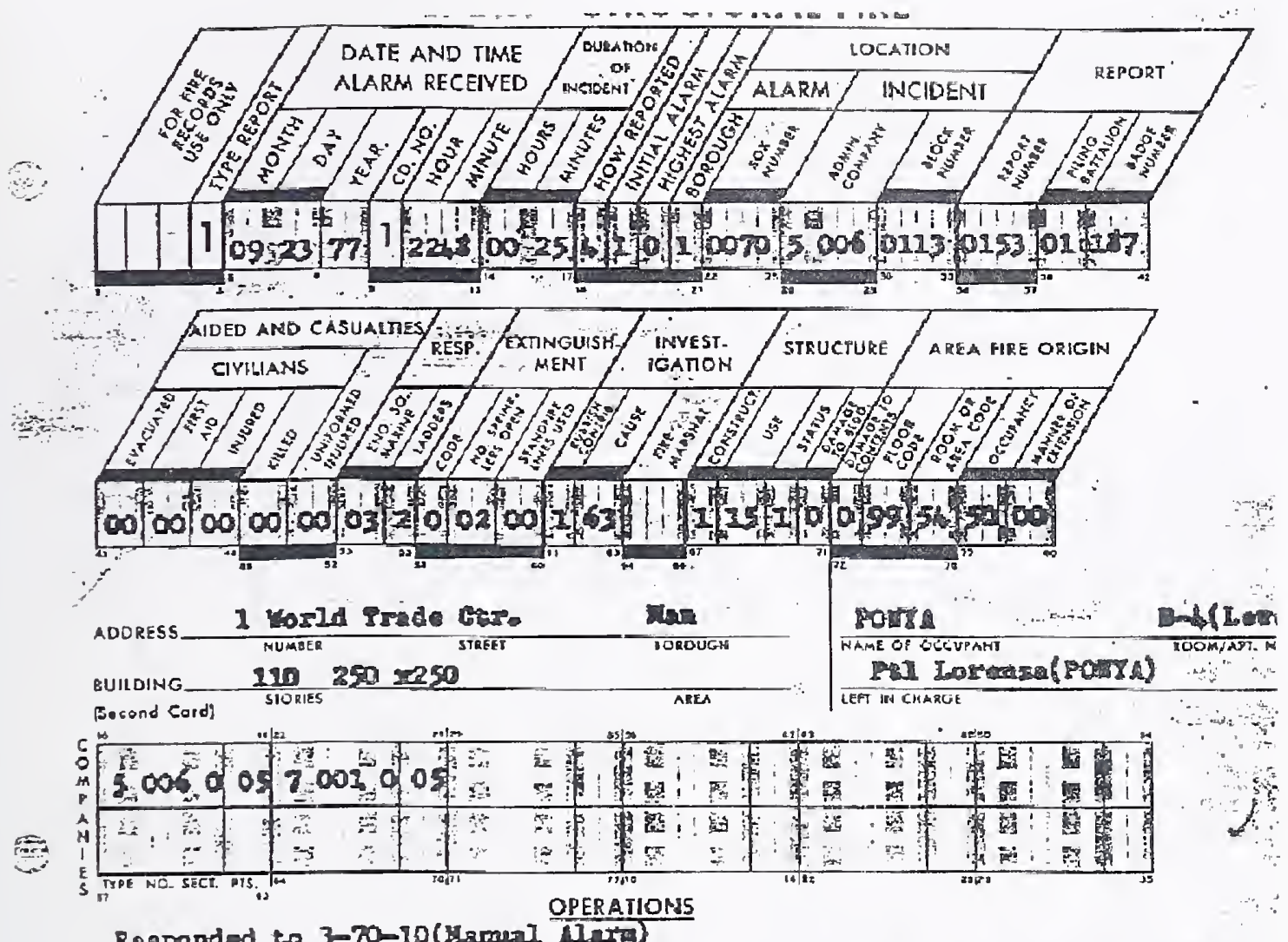

Eesponded to 3-70-10 (Hameal Mitra)

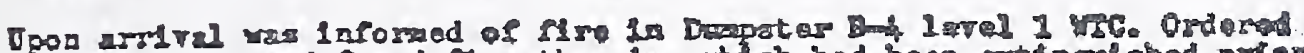

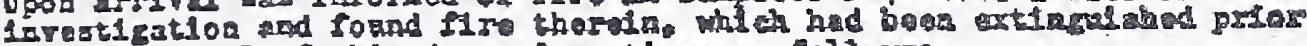
to th arrival of the dept. Operretion as follegse

8.6 Aollad up 2angths stood rast.

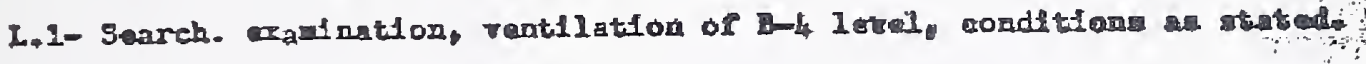

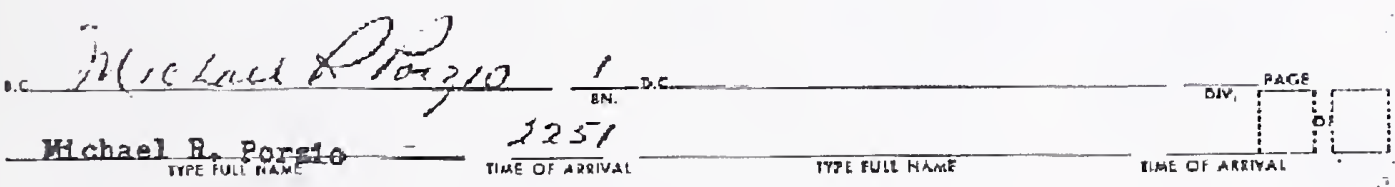

ADMINISTRATIVE COMPANY 


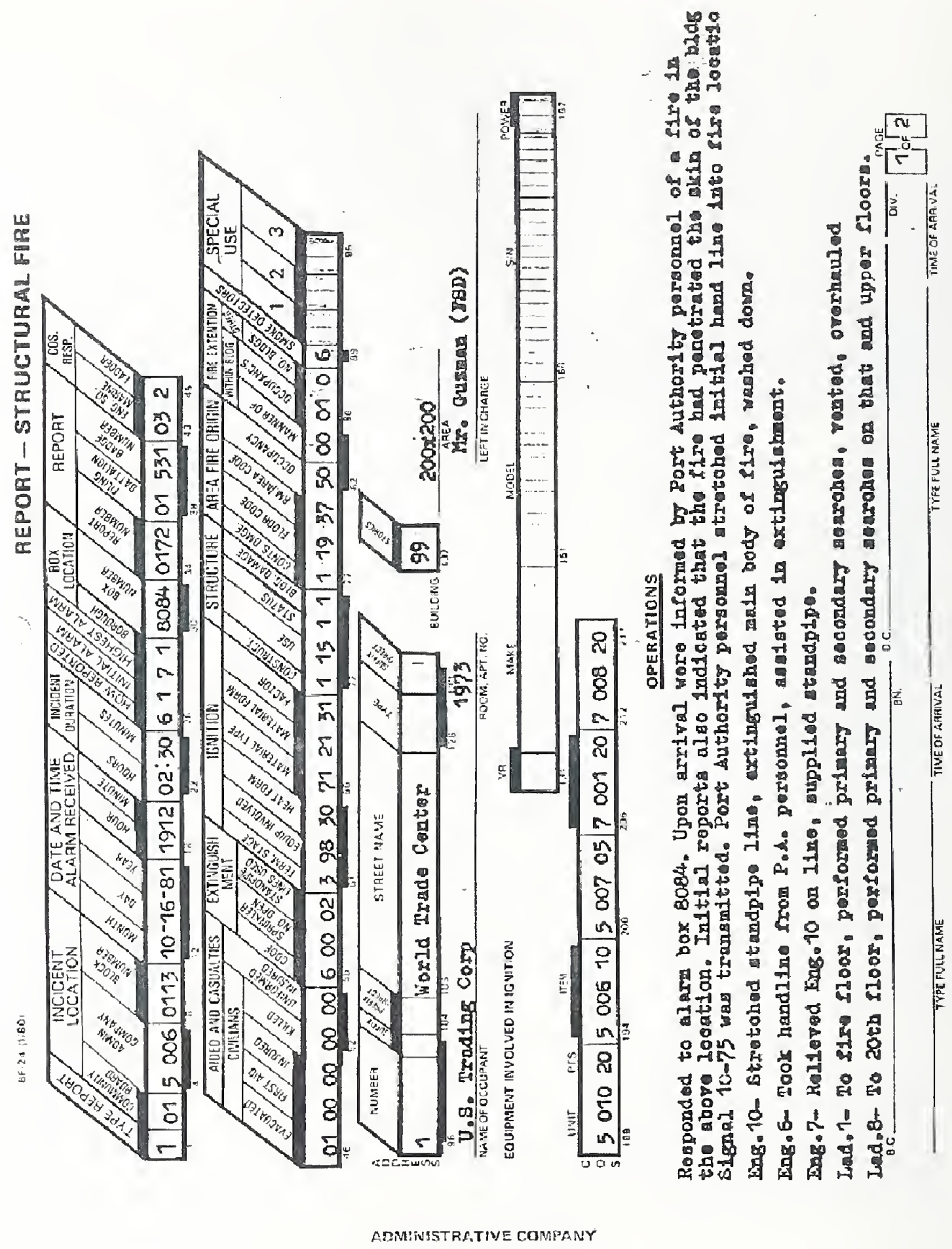




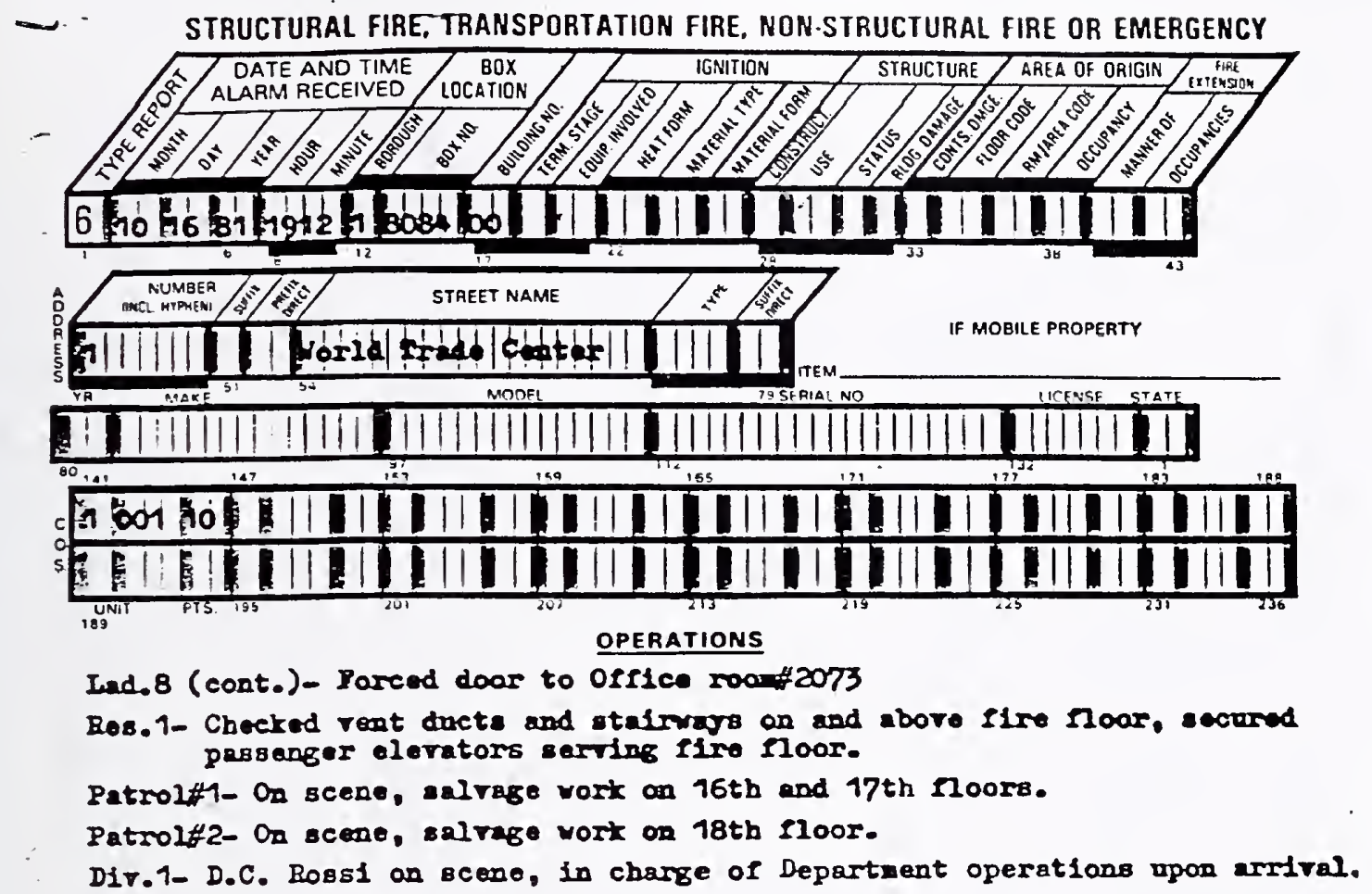

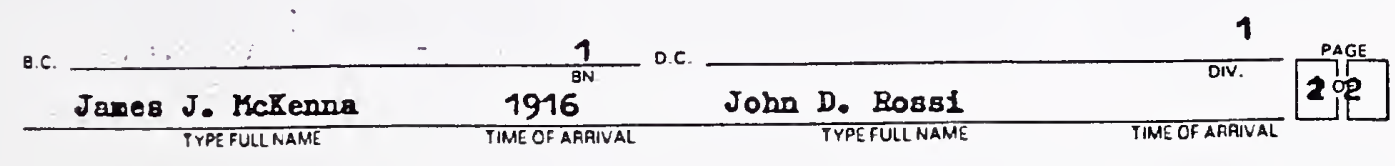




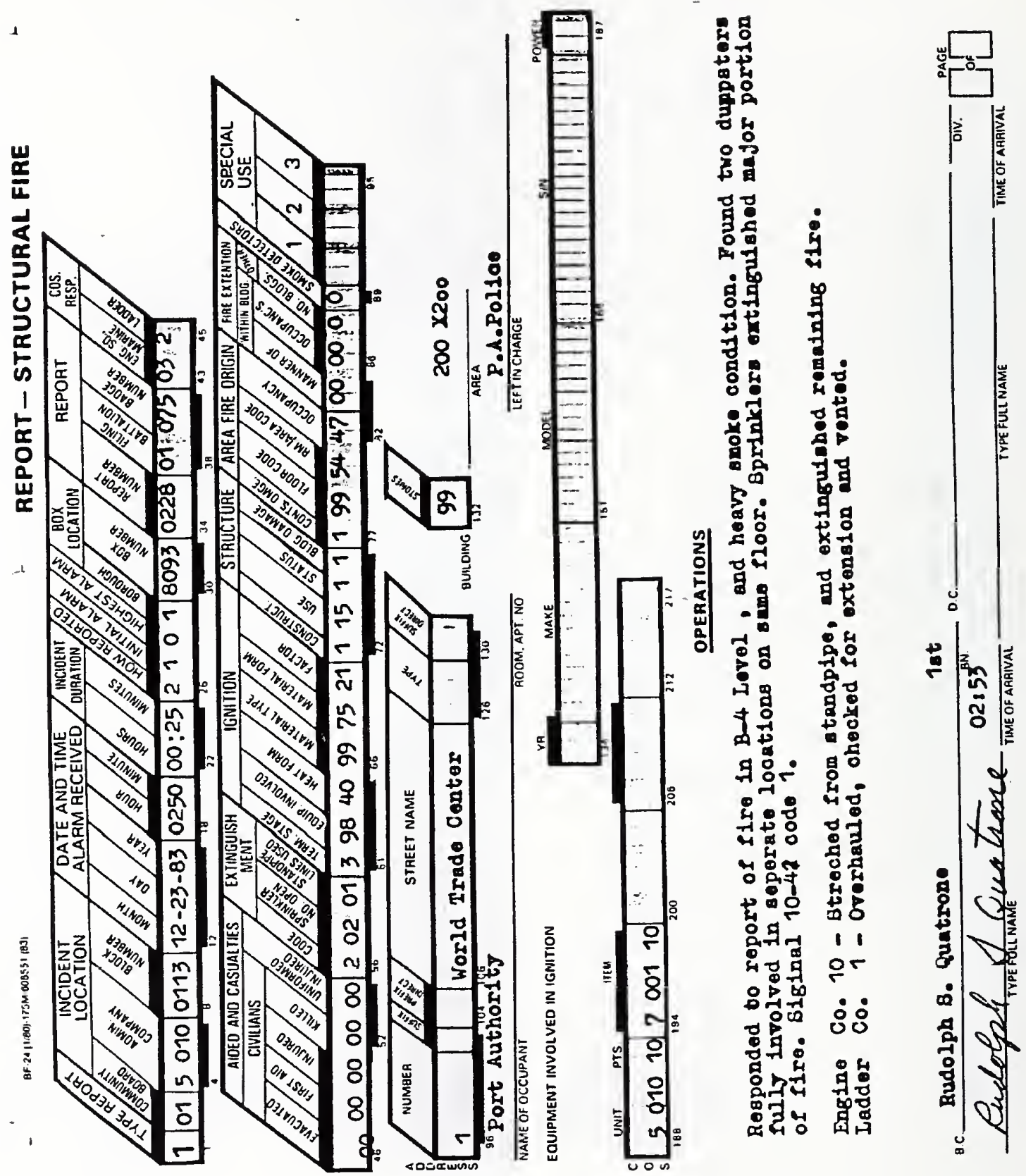




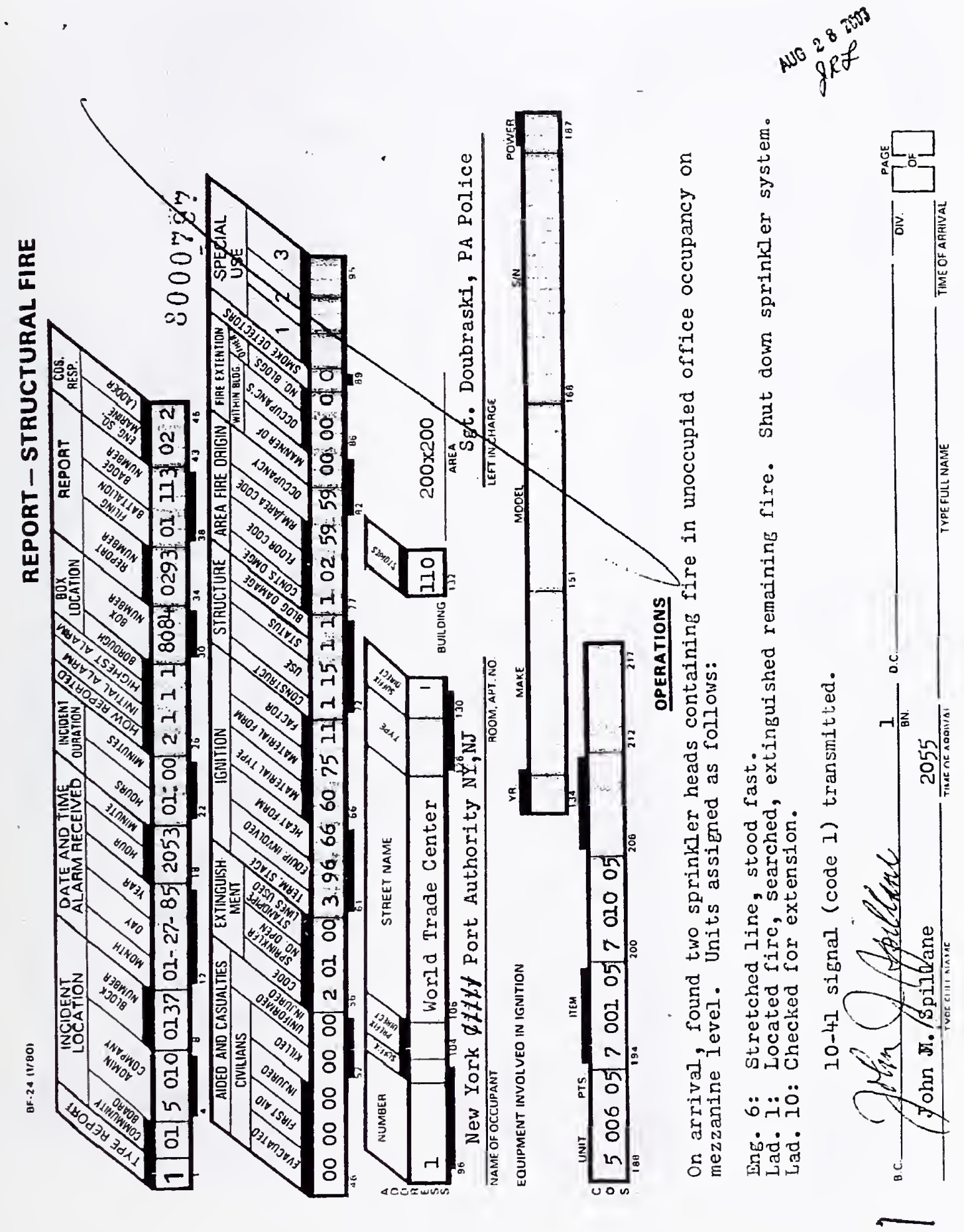




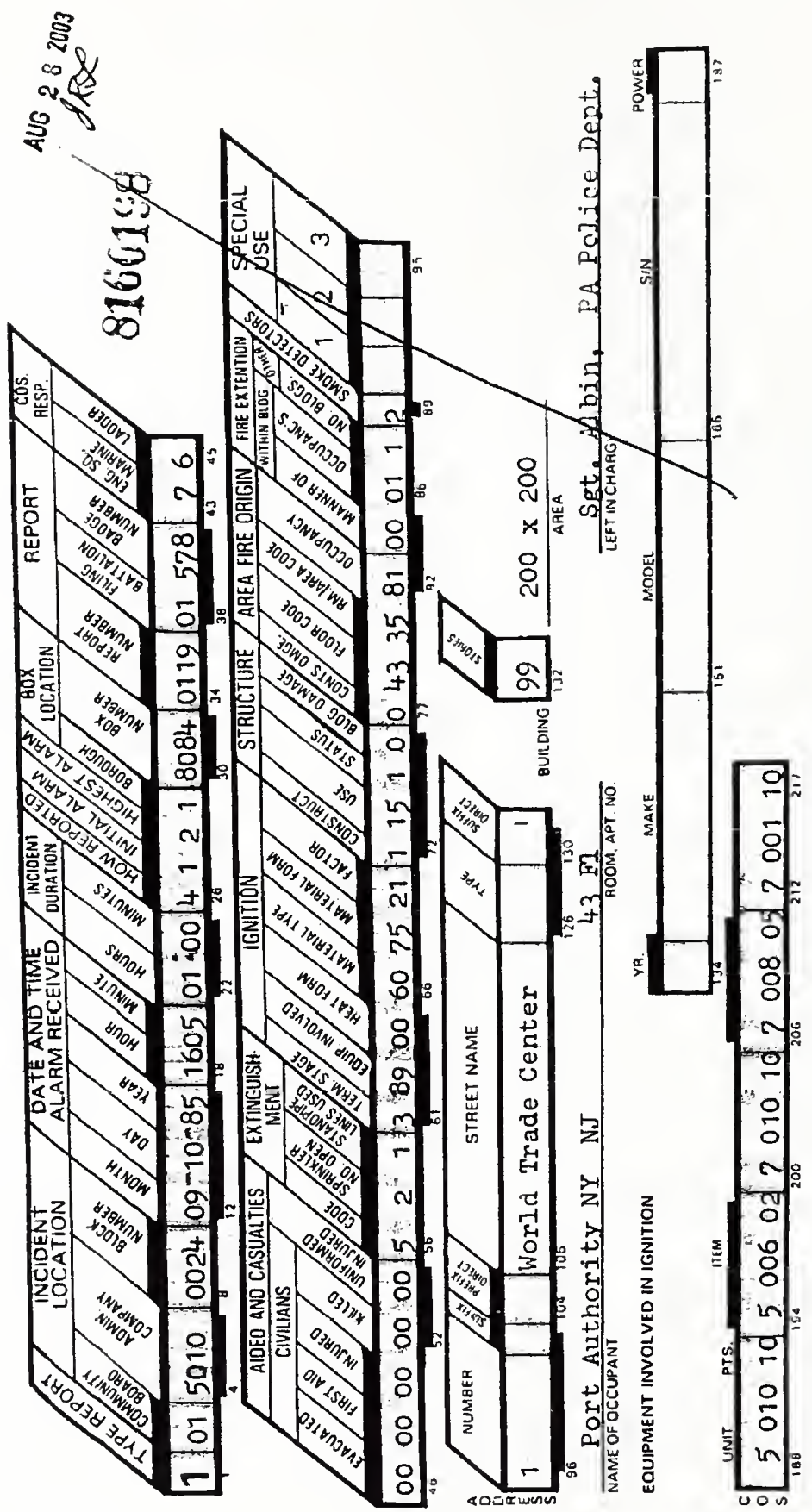

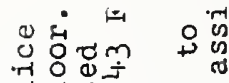

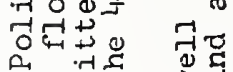

m实

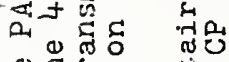

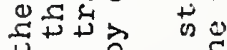

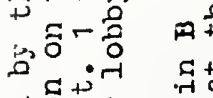

踏

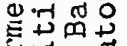

कृ

4 40 व

. 0 엉

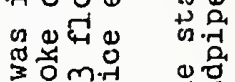

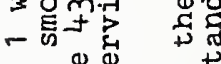

$\because 0 \stackrel{0}{0}$

式赶我

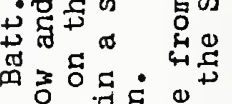

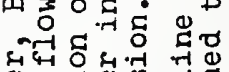

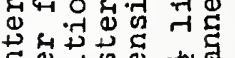

कृष्ठ

일 궁

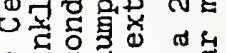

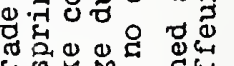

है

部 以

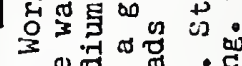

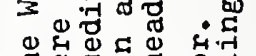

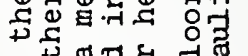

फ तु क्ष क्ष

काष्ठ

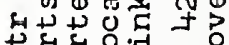

U 영대

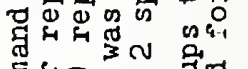

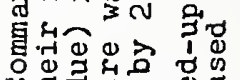

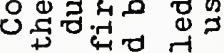

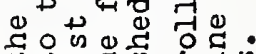

एक

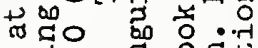

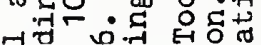
तु 웡웡워

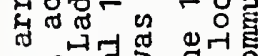
ॠ जत्

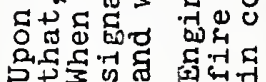

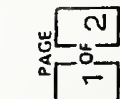

-

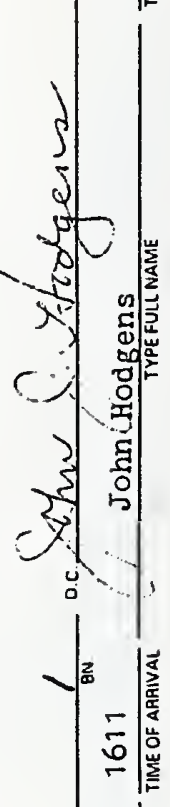

FIRE RECORDS 


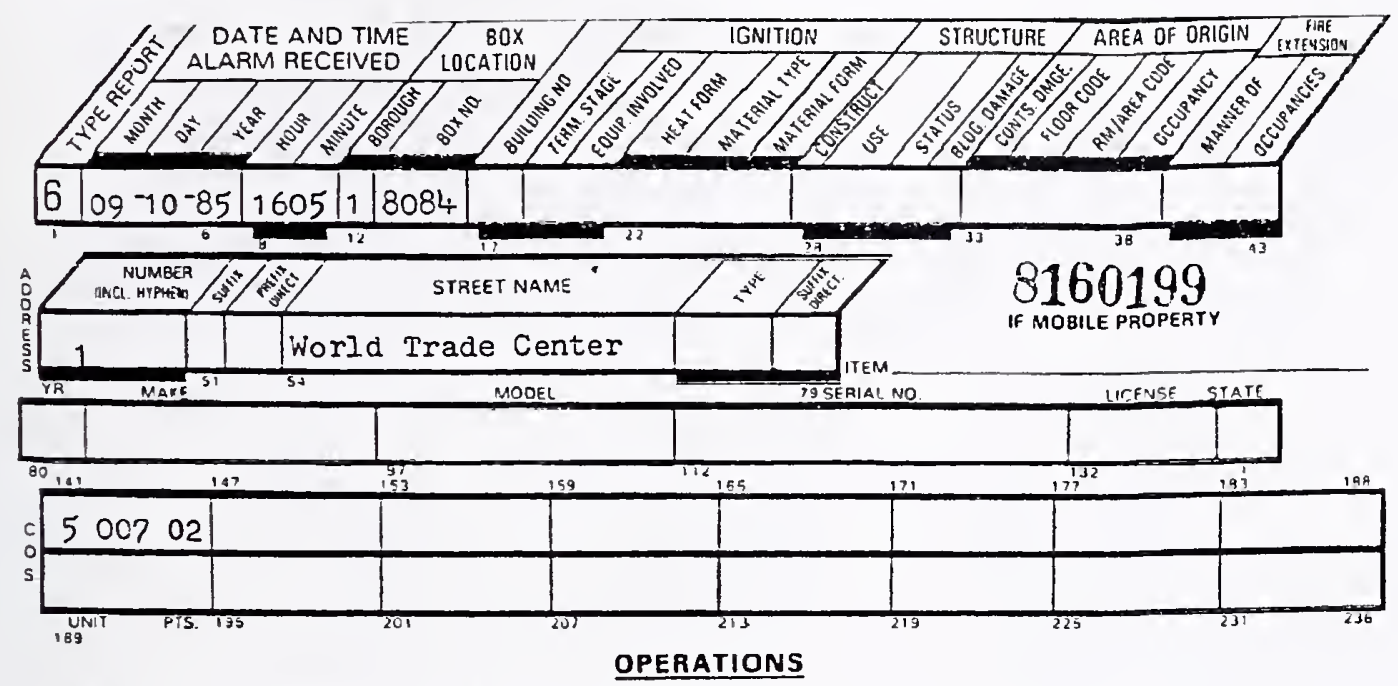

Engine 6- (teamed with E.10) Assisted E. 10 in Standpipe stretch.

Engine 7- Manned the Standpipe phone in Stairway B on 1 st F1., relayed messages to and from Command Center

Ladder 10- To $43 \mathrm{fl}$, stairway B, found fire, searched $43 \mathrm{fl}$ (negative) made neesessary examination and overhaul of fire area.

Ladder 1-to $44 \mathrm{Il}$, searched for fire, smoke extension, searched for troubled occupants (negative) Checked top ten fls (101-110) for smoke (Neg.)

Ladder 8- Made secondary search of $43 \mathrm{fl}$ (negative). Searched fls 44 to 49. Searched affected service elevator ćars \#17 \& 29.

Batt. 4 - Set up operations post on $43 \mathrm{fl}$. Communicat ed with Command Center, supervised operations of units on 43 and $44 \mathrm{fls}$.

E.4, E.3 (HiRise Unit), L.15 \& Res. Co 1 stood fast, took up.

Brooklyn Cos responding on supplemental Boxes 9031 \& 9032 at 1617 hrs Batt 31 ,

Batt 32, 2. 205, E. 279, L.101, L.110 stood fast, took up.

F.C.U. - Established Field Hqtrs, Monitored Communications.

Sprinkler heads (2) replaced by P.A. personnel.

Signal 10-41, Code 1 transmitted, Evidence of separate, previous fire reported to FM NaCaffrey.

Responded to Scene: AC Matthew Farrell, Manhattan Borough Commander

\begin{tabular}{lll} 
Signal & Time & By \\
\hline $10-84$ & 1609 & E.10 \\
$10-76$ & 1615 & Batt 1 \\
P.W.H. & 1627 & Dir 1 \\
U.C. & 1640 & Div 1
\end{tabular}

$x$

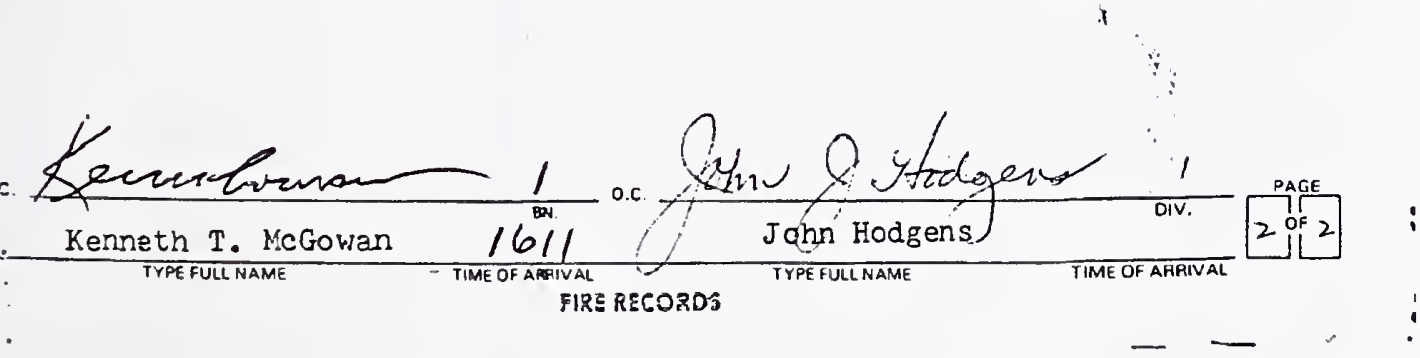



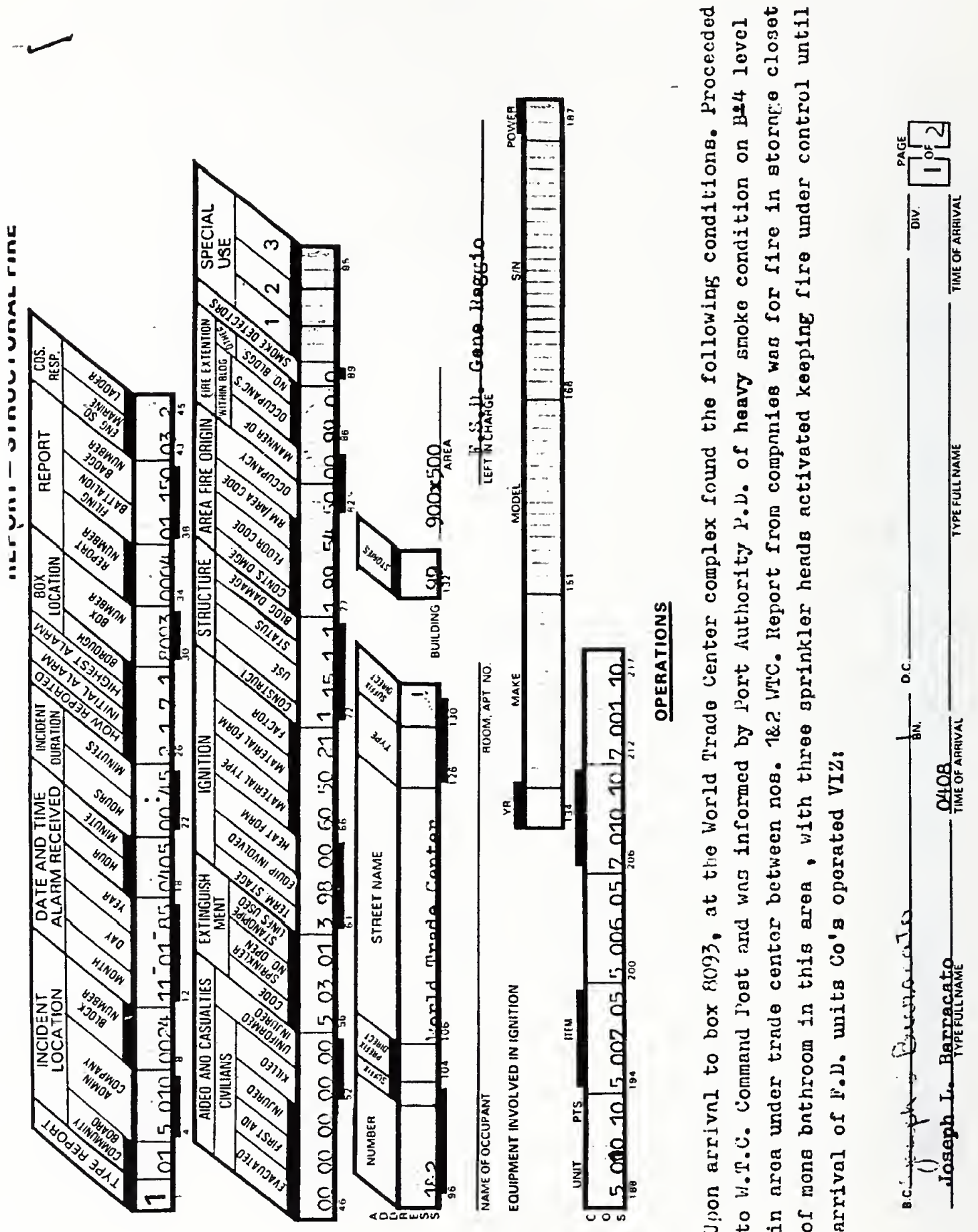
NETUNI - MUUIUUNGL UAIH

- STRUCTURAL FIRE, TRANSPORTATION FIRE, NON-STRUCTURAL FIRE OR EMERGENCY

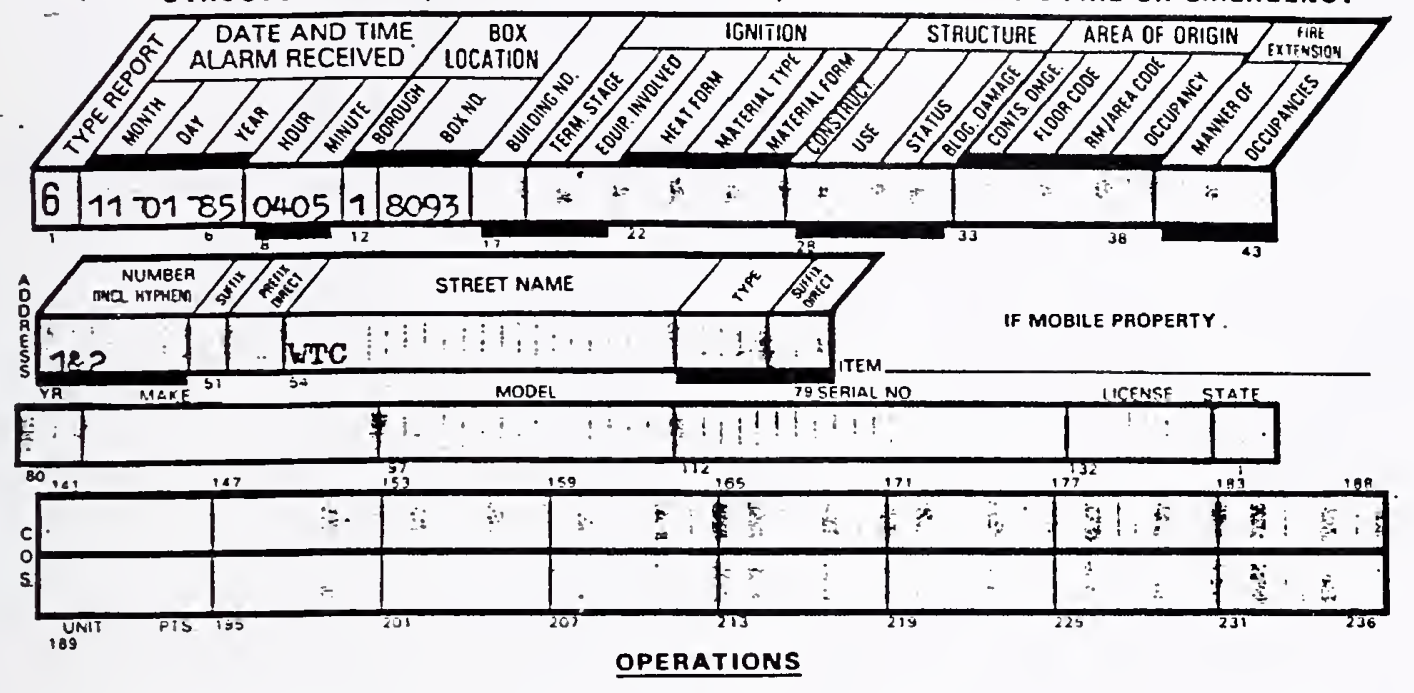

E-10 stretched line into fire area from standpipe and extinquished all remaining in closet area

in Etretched line fron opposite side of fire and stood fast

- 6 assisted 210 in stretching line and relieved on line and then washdown

I-1C Found fire and perforted necessary VFa and overhaul in area, mace primary and secondary search, then up

I-1 performed necessary $V i$ and checked for possible extension in surroundine areas

$\begin{array}{rr}\text { Fimes: } 10-84 & 0408 \\ 10-75 & 0410 \\ \therefore 11 \text { l:ands } & 0425 \\ 10-41-1 & 0425\end{array}$

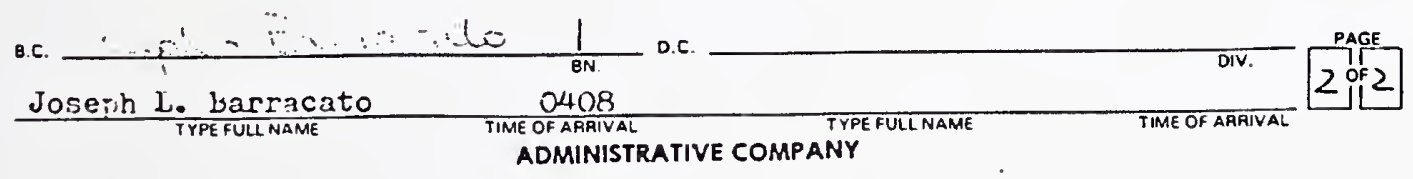




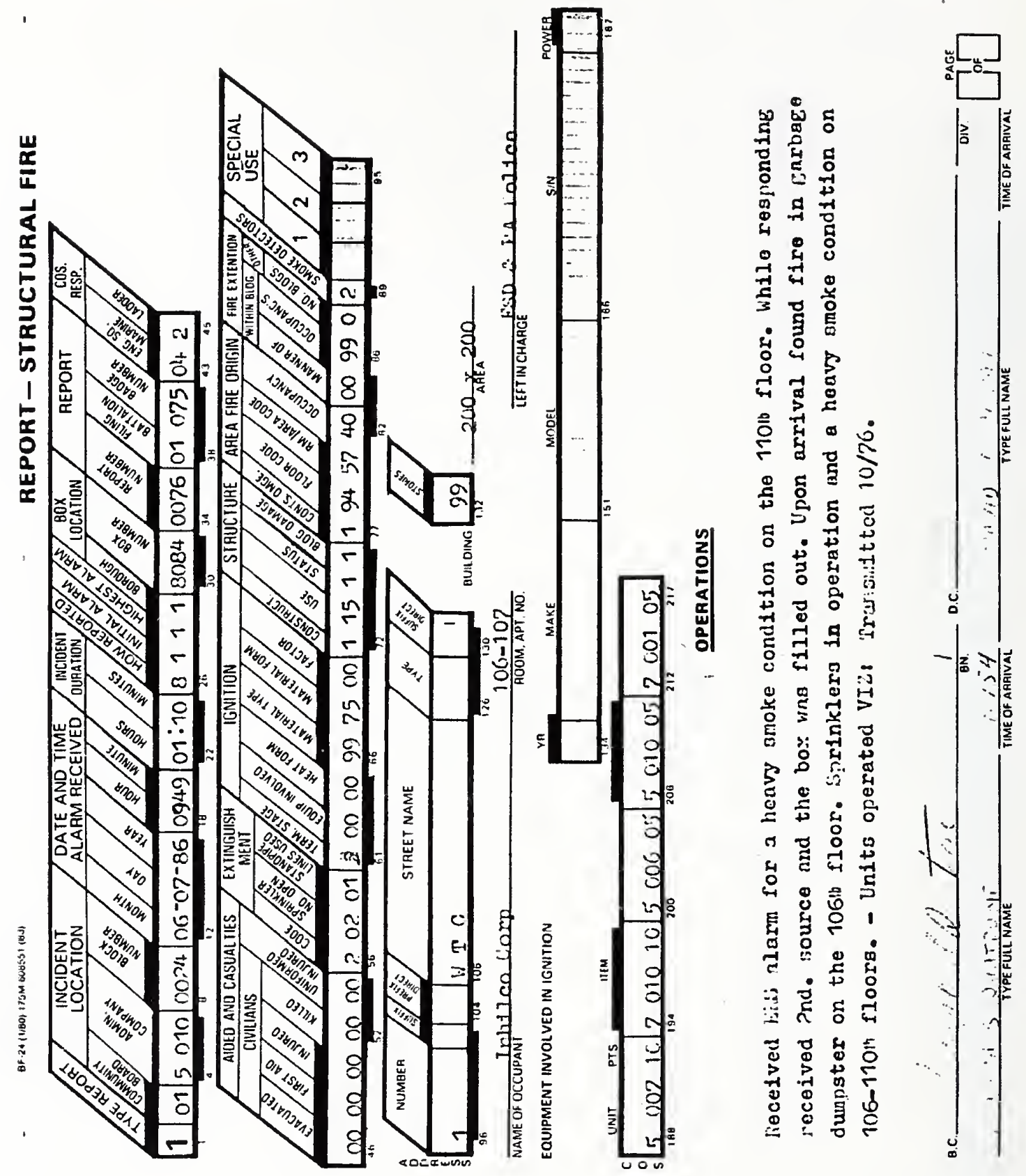


KEPORT - ADDITIONAL DATA

STRUCTURAL FIRE, TRANSPORTATION FIRE, NON-STRUCTURAL FIRE OR EMERGENCY
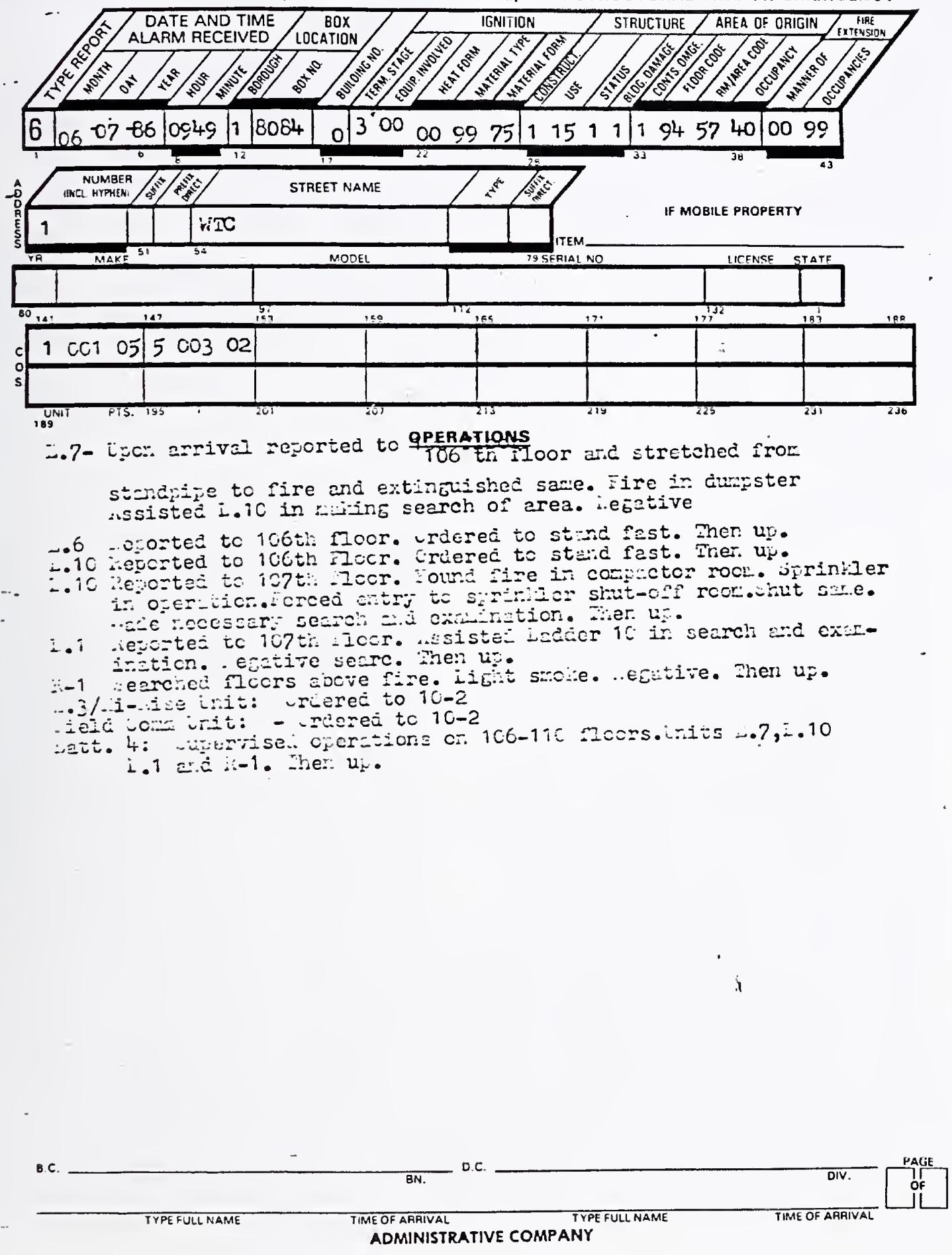


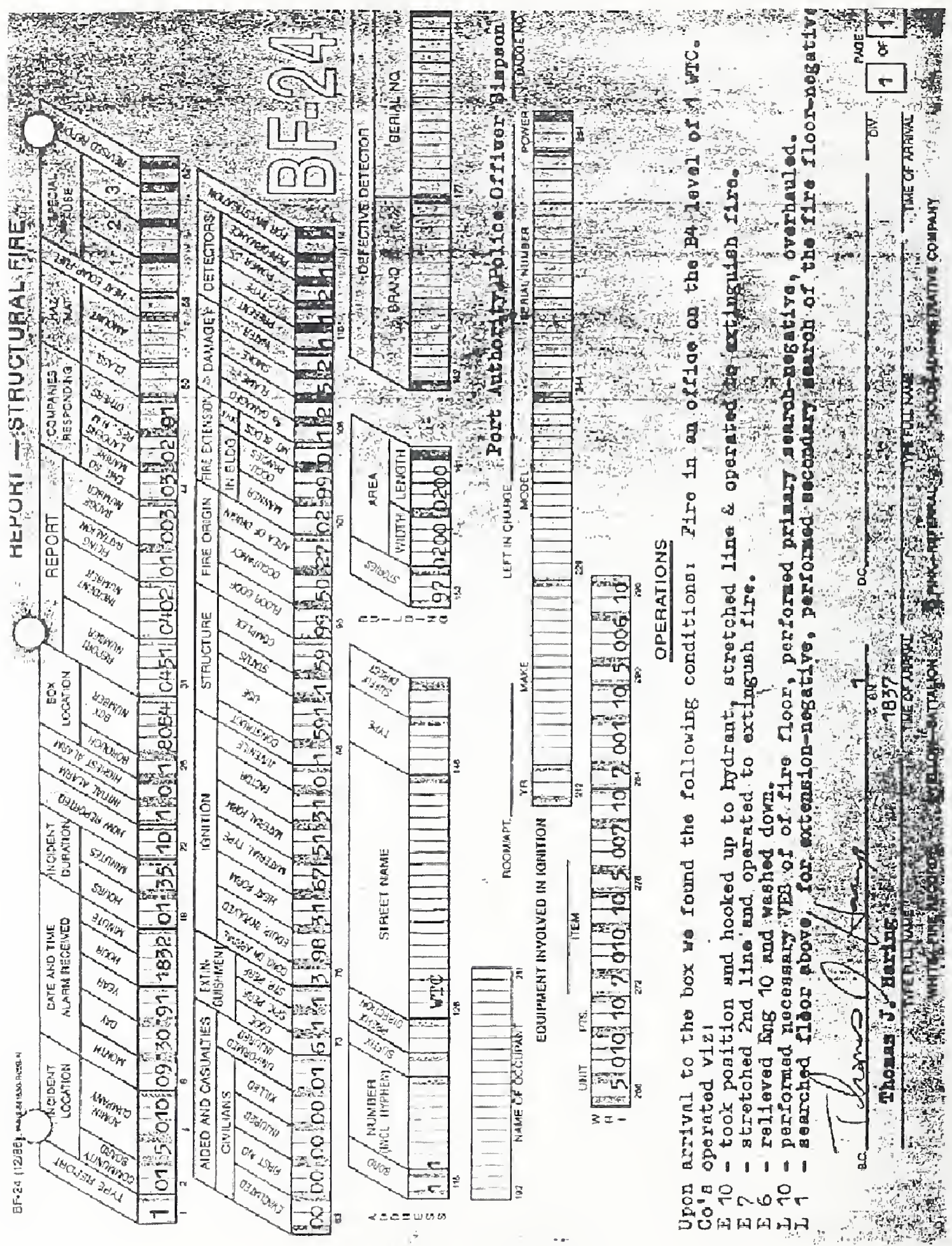




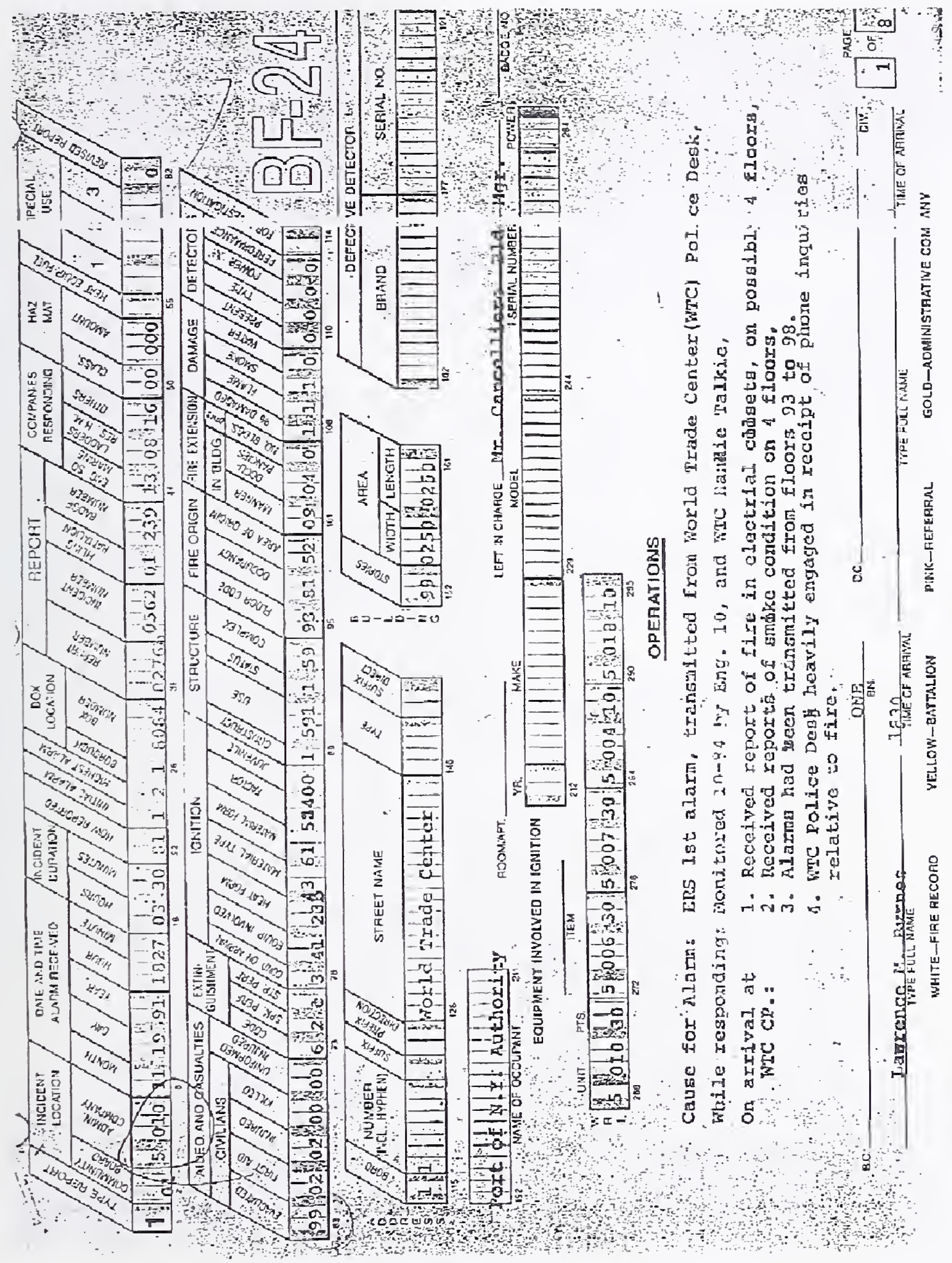




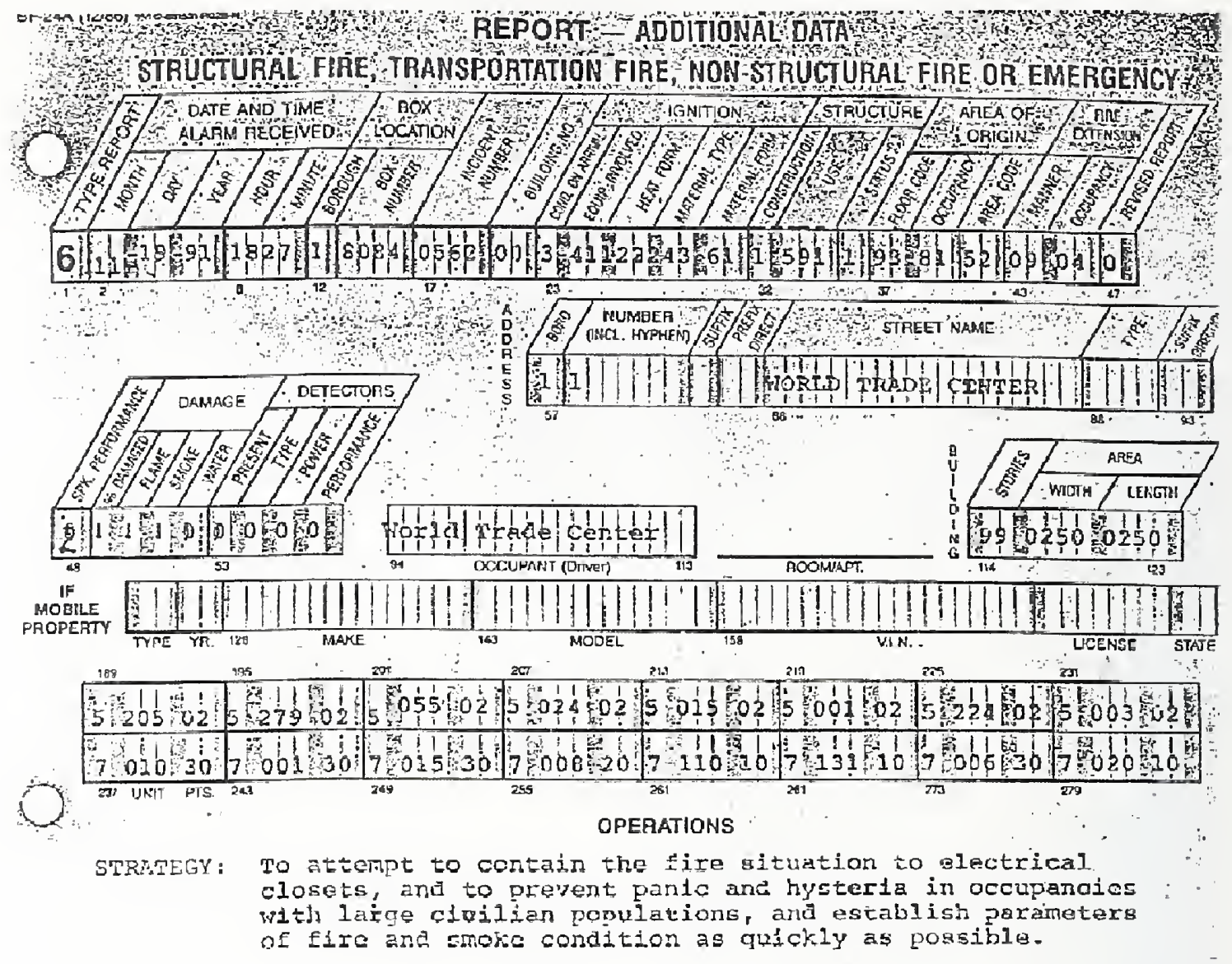

Action taken:

1. Bn axival at Fic comant post, aszuned comand of Fire altuation.

2. Eominicated with landar co. 10 mho confined fire situationon floors $93,94,95$ and possibly on 96 ,

3. Ordered the transmision of signal 10-76 isased on: potertial for large volume of Elre, height to fixe axea, time involved in units response, difficulty in cffecting good voice comnumieatloss in building,

4. OrJcred Manhattan Dispatcher to 3lext 10-76 Exrivirg units to assemule in tobby stiging. area, pending unft assigureyt,

5. Ordered fingt alarm units to conouct aisigent search.

6. Ordered Ist alarm Inglne compenics to stetch from the "A" stairwell, but not to use water until confrimation

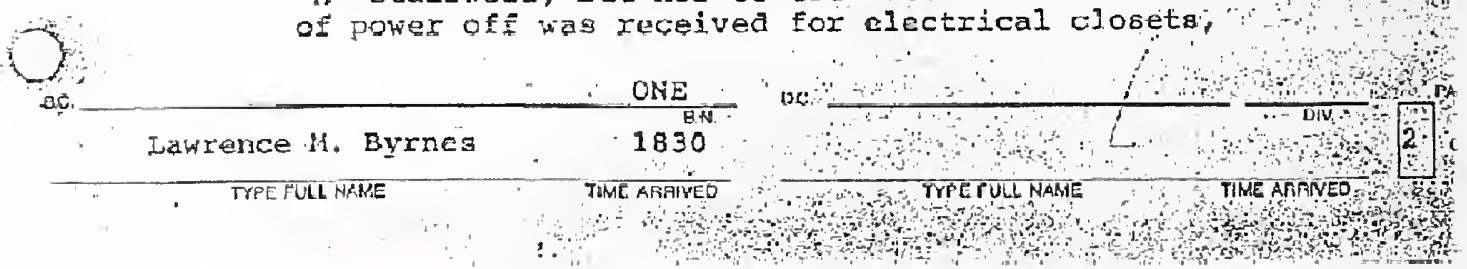


BF24A-1206 wand 3 A REPQRT - ADDITIONALDATA

* STRUCTURAL FIRE TBANSPORTATION FIRE, NON-STRUCTURAL FIRE OR EMERGENCY

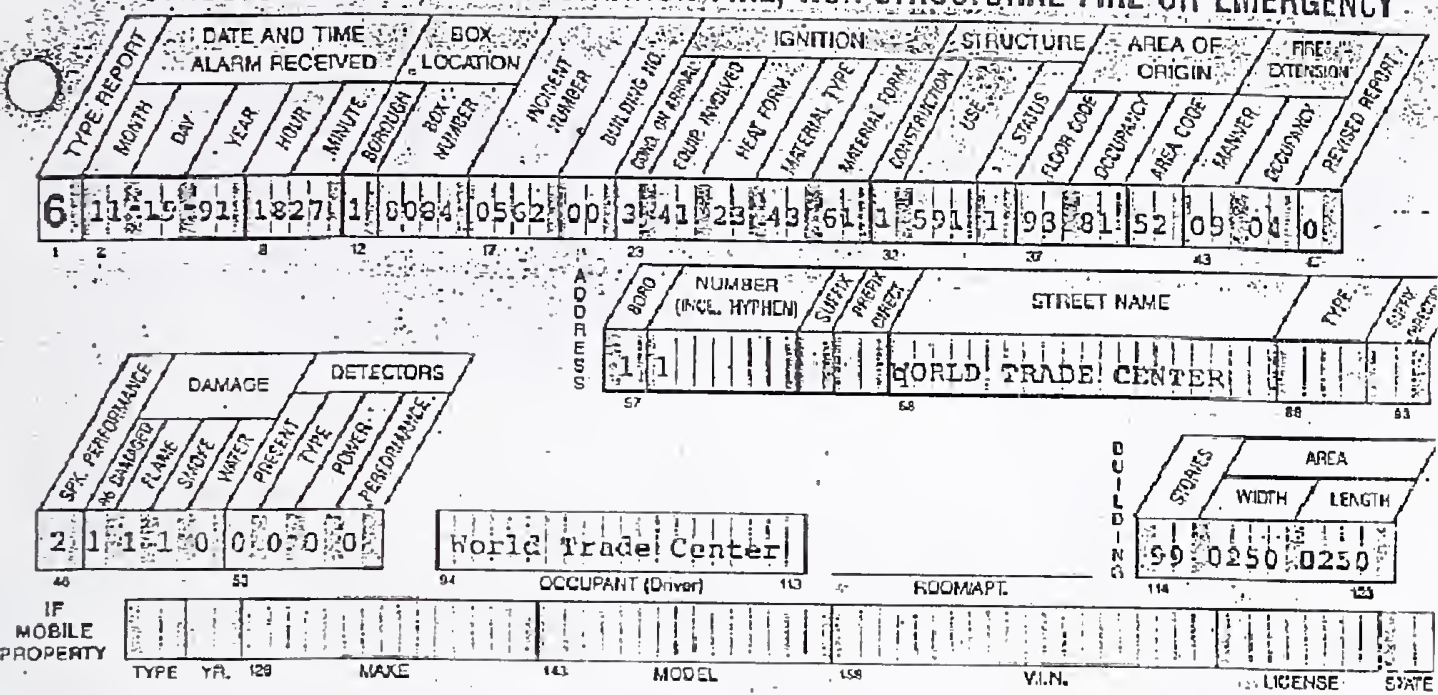

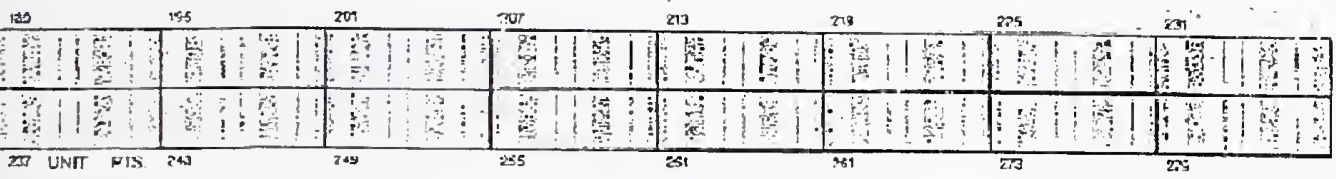

OPEHATIONS

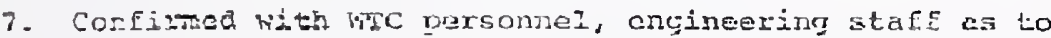
time frame bor electrital power inoval for effectea floors,

E. Lrenied bre Fulice xequest to initate general evacuation of Eloors "9j to rooz level", based on unkrowns of: a.smoke corditions,h. stuir availability, c.norbess of people that may bo invoived,

9. Upon rotification by WyC Enginearing uta

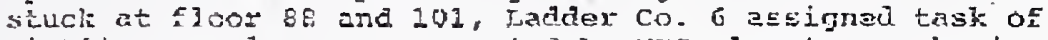
victet zenoval, as accomeanied by HrC elevotor mechende.

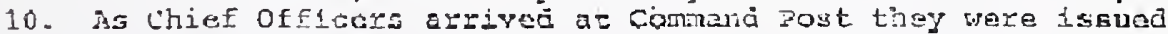
VTC Handie ialkis and theì Assignments,

11. Called for wis rosponss for reported burn victin /ncchanic with farial burns and for pesible nedas,

12. Primari search of loper flours orovea to je time consuning due to large floor area and linited Handie matie capabalities, conducted by list alem trucies.

13. Rescuo Company I assigned to follow up cccondary search of Resuo company 1 assigned to fol 10 wa cocondar.

14. 10-75 signal proved very efidctive, proviled trinediate response of chfef officers to ihitiate various scctor controla.

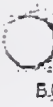<smiles>[AsH2]=[AsH2]</smiles>

T,iwirence M. Everses

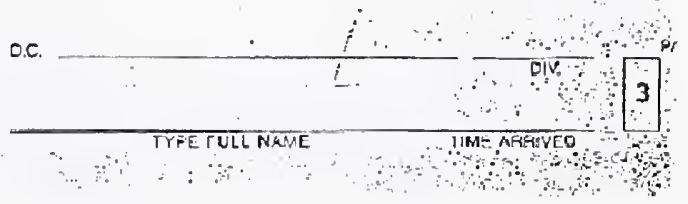




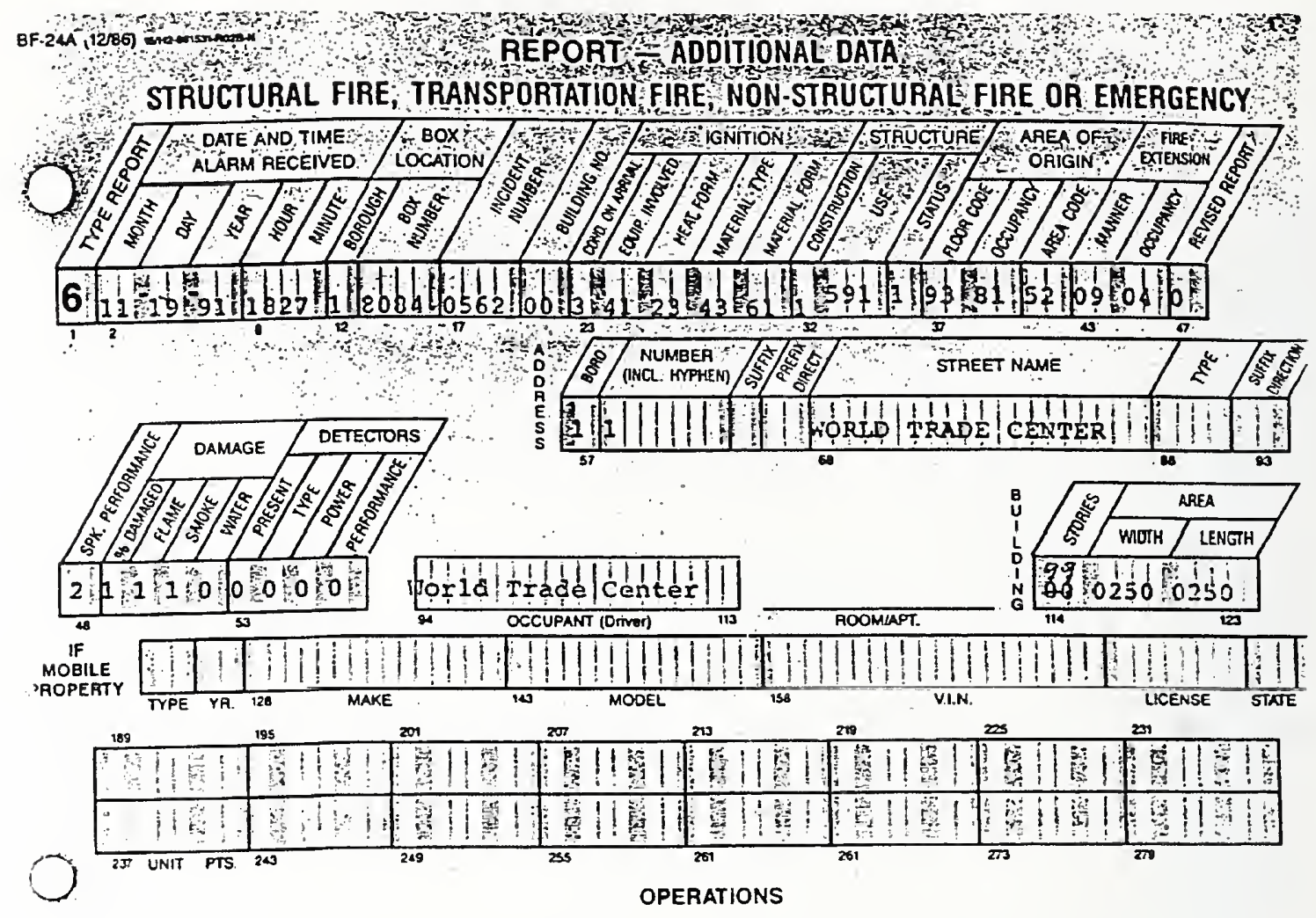

Chier ofliceis at scene:

DAC. E. Eutler,.
JC. H. Heyers
DC. V. Dunn
E.C. I. Ryrnes
B.C. R. Ardisson
B.C. Davison
i.C. Roche
B.C. Shelley
B.C. Wagner
B.C. Dawe
B.C. Cesark

Cis Duty,

Div. 1,

Div. 3 ,

bett. ONE,

Batt. 2

Batt. 4,

Batt. 6 ,

Batt. 31 ,

Eatt. 32,

Batt. 7,

B.C. Cesark

Safety Battalion.

Personnel present at Scene:

R. Sclibizer Fire Com. Laison,

D.C. Basile Eils Supervisor,

EYro $\because \therefore$.

Lt. Valle HYPD Pct. 1 supervisor,

Mr. T. Cancelliere iTC Blag Mgr.;

(2) Representatizue of Salvation Armir.

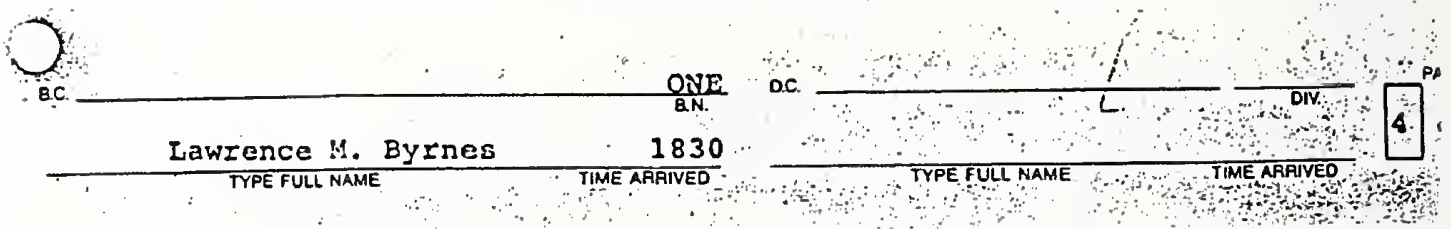




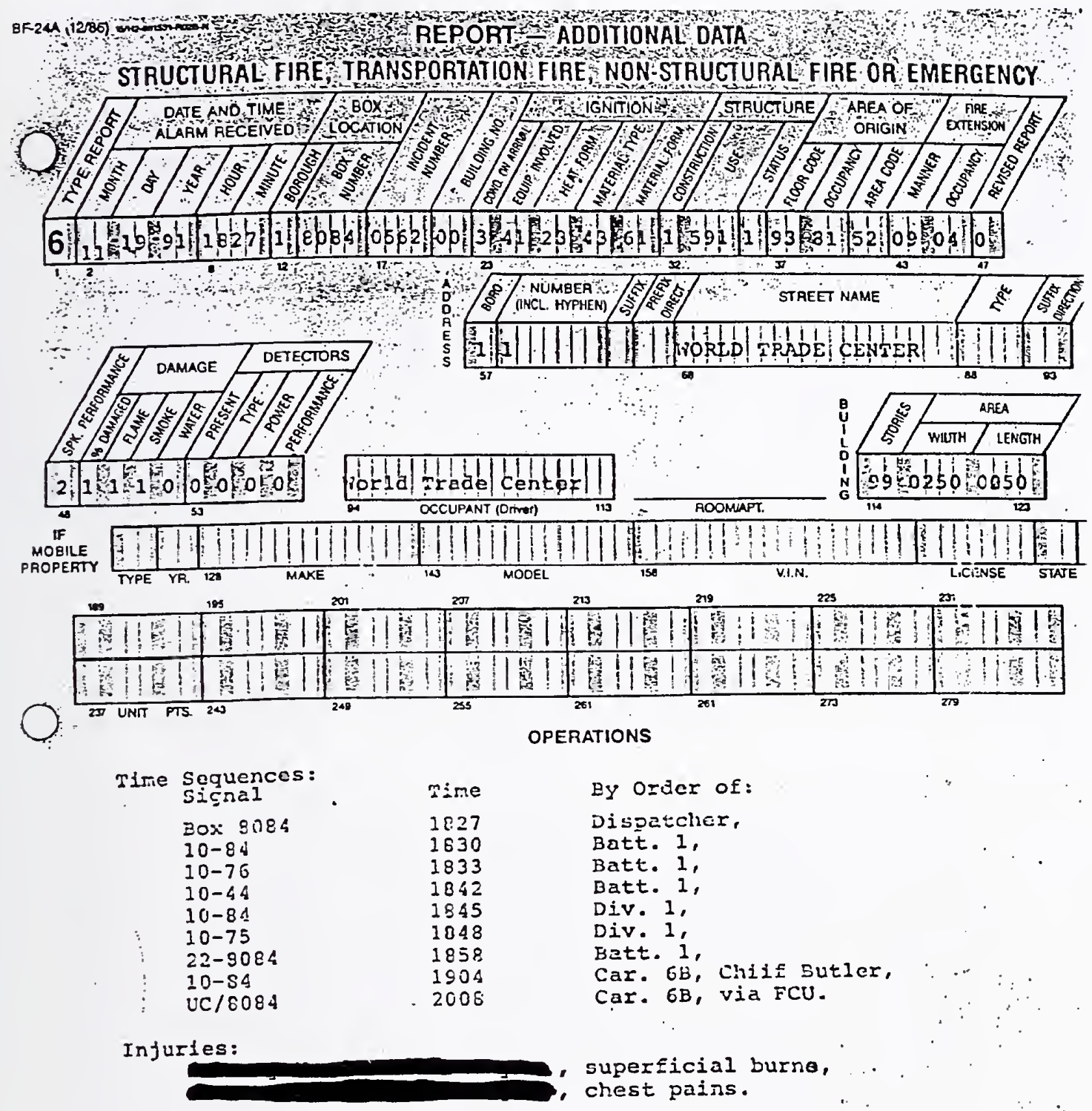

Unit C1tations to ge subnitted for Ladder Co. 10 and Engine Co. Io.
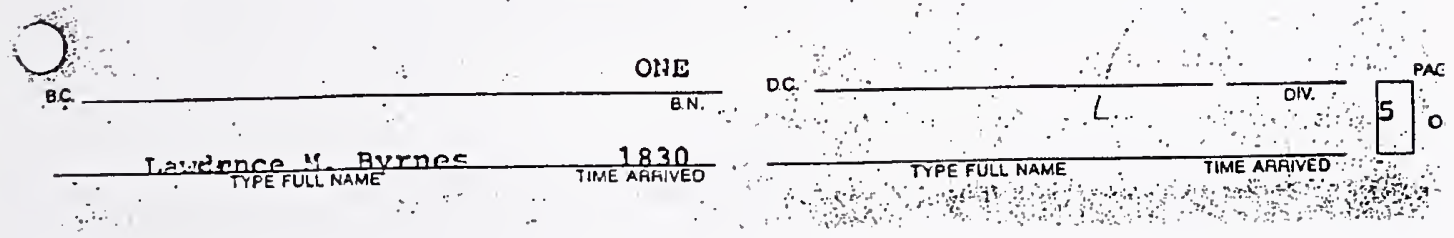


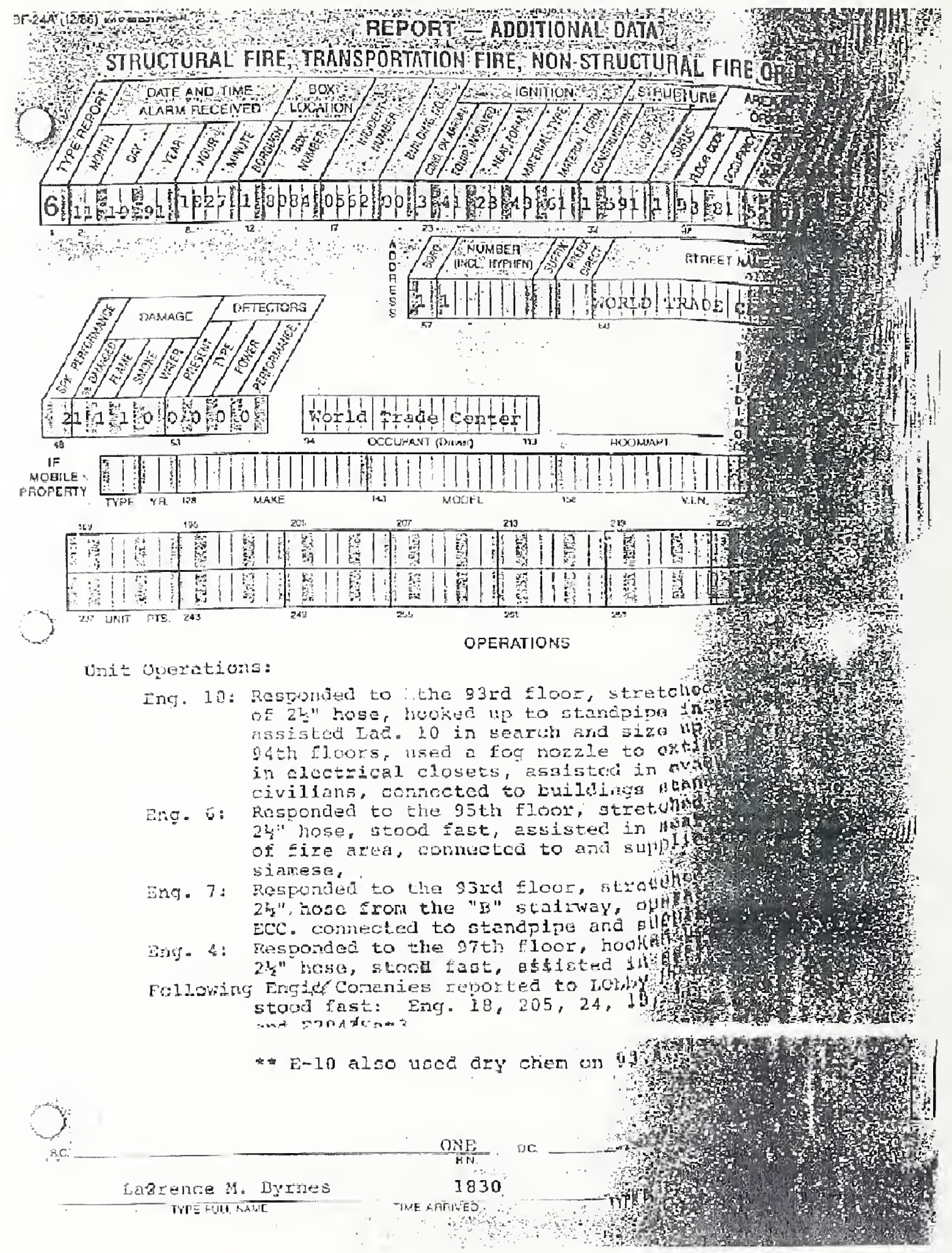




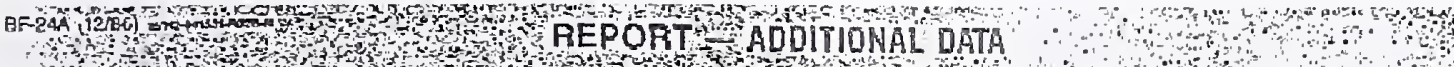
STRUGTURAL FIRE TRANSPOATATION FIRE NON-STRUCTURAL FIRE OR EMERGENCY

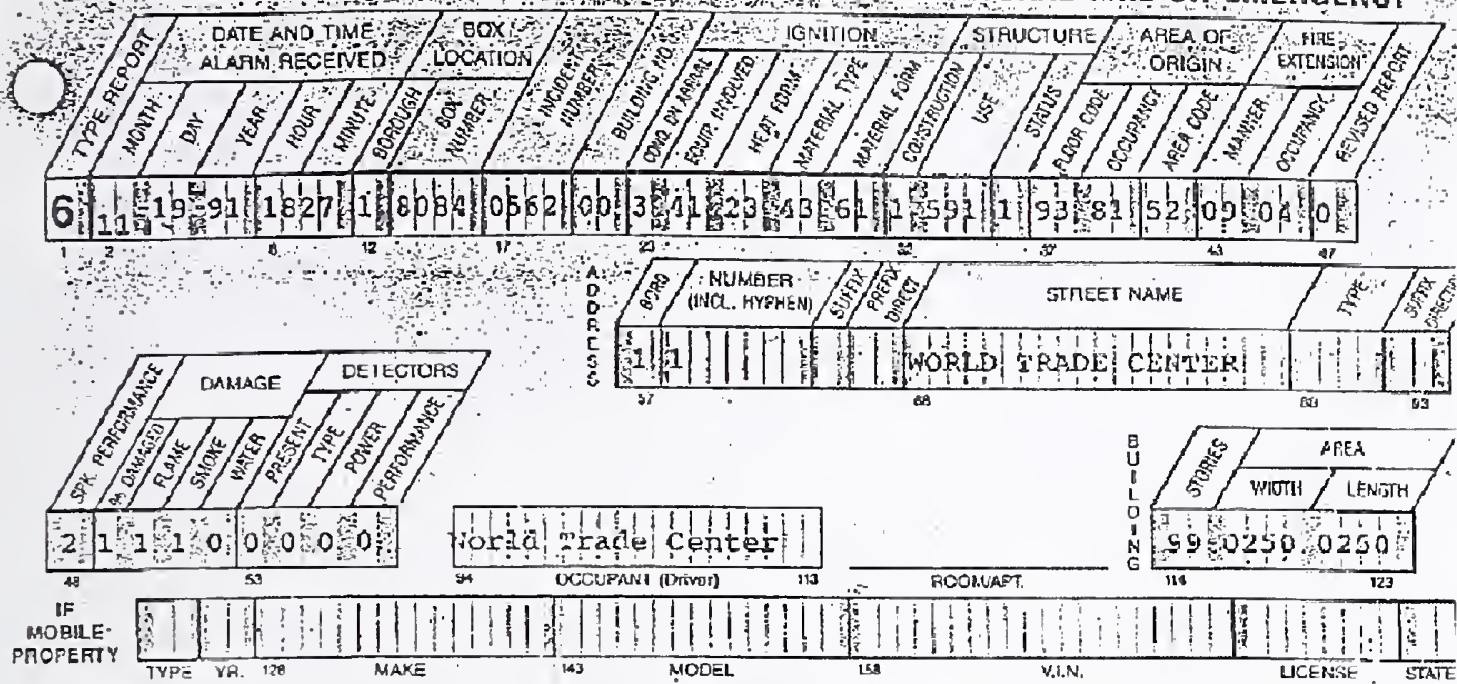

\begin{tabular}{|c|c|c|c|c|c|c|c|}
\hline 189 & 185 & 201 & $x+2$ & 213 & 213 & 83 & 251 \\
\hline 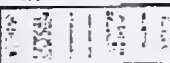 & 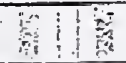 & 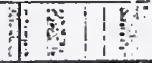 & 10110 & 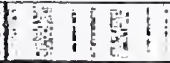 & 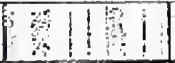 & $=11 \% 1$ & 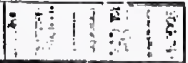 \\
\hline \$ & 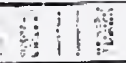 & 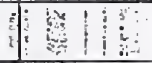 & 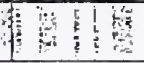 & 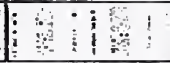 & $\begin{array}{lllll}0 & 1 & 0\end{array}$ & 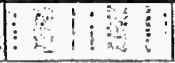 & 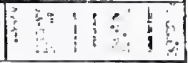 \\
\hline
\end{tabular}

OPERATIONS

UHIT OPERATIONS cont:

Ixa, 10; Canducted primary seerch on iloors 93, 94, 95, ancl

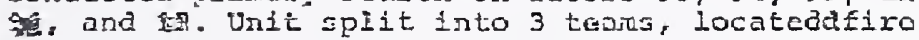
conditlon in electrical distribution ciosets, calied for placonent of hand lines, conducted opening up uneratious on 94 th EIoor to cheak for fire crtanston, cvacuated unknown rnerber of civilians from floors 94 through 25 suring inftial operations,

Iad. 1: Conducteil primary searcin on 93 rd tloor, conducted sccondarg, sarch on Eloors 94 to 101 , proved to be nerative, conducted survey on floors 81 shrough 92 for water danage to electrical closets due to Hhadline use, regative.

Lad. 15: Conducted peinary and eecondary seetched of rloors 93 to 97 , nugative results,

Lak, 8: Conducted exeuination of floozs 92 to 101 fne Dosibie fire extcnsion from electrical closets..

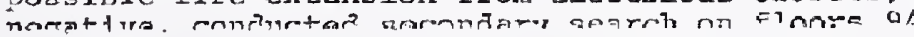
and 95 .

Lad.120: Iexformed Eecondary search of floors 96 through 110; negative results,
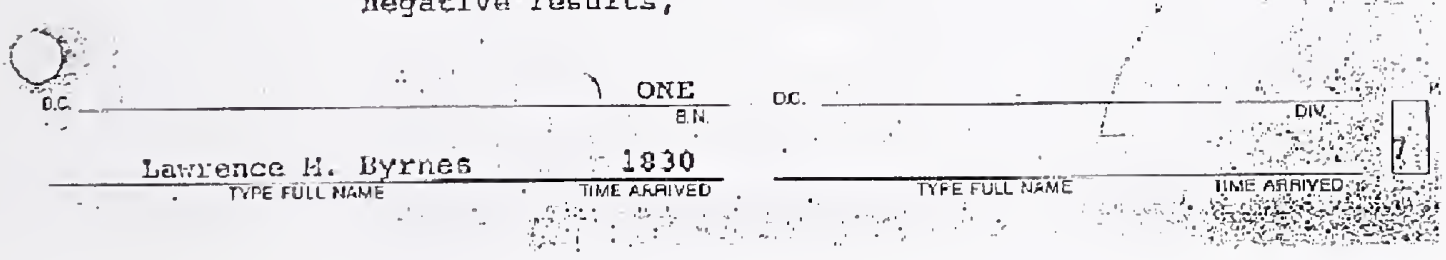


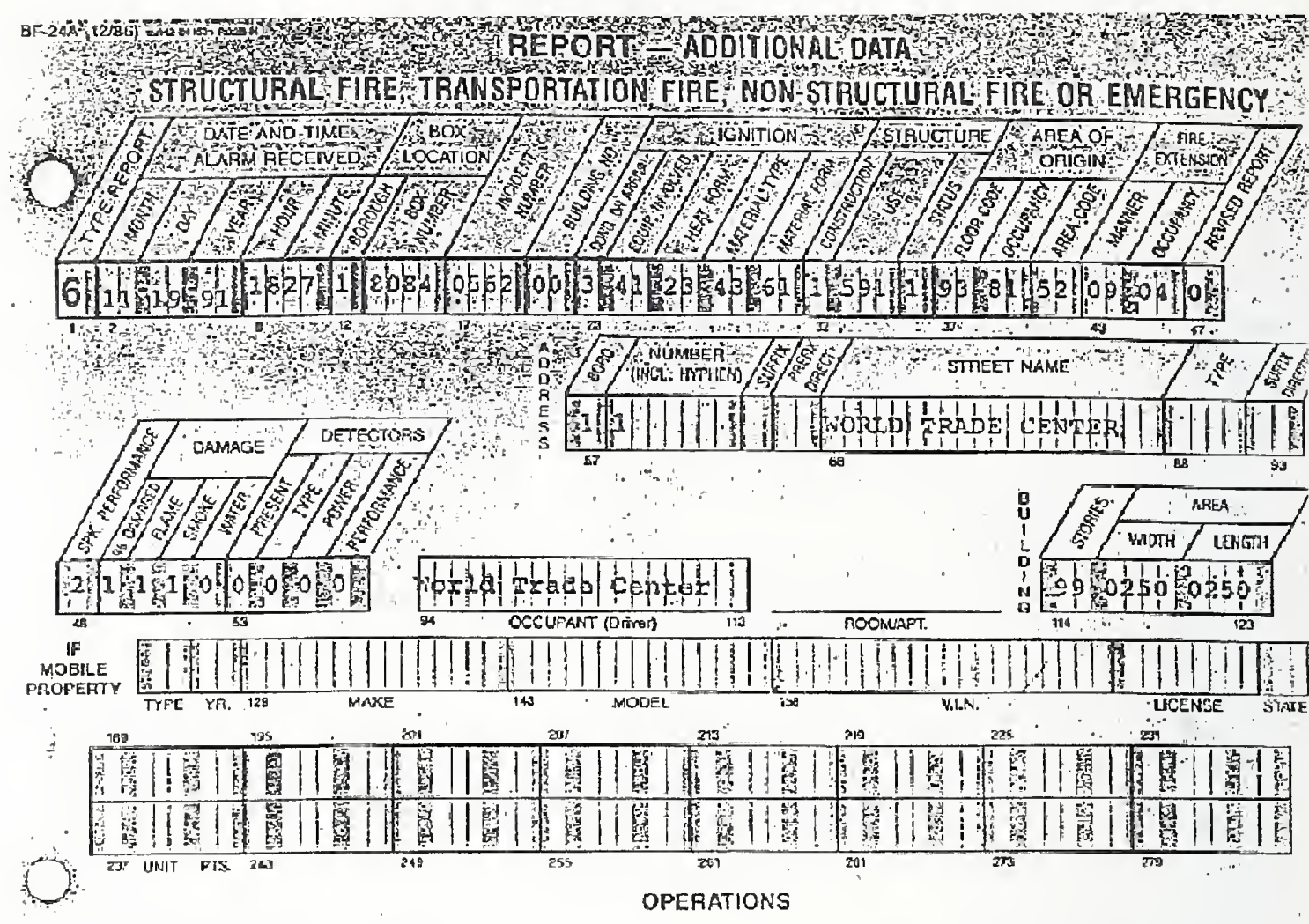

Uoit Operations Cont:

5ad. 6: Conductch scnonciaxy eexich 66 floors 105 to 110 , negativg esults, removed (2) civilians from stalled elevators on the aEth and 1015t floors, no injuries to civilians, or danage to bollding,

Lă. 20: Conúucted secondary seerch of floore 40,99, and 100 negative resilts,

Lad.131: Conducted search of floor:3 105 to 110 , with negative eesulte.

Fleld conumications unt: sat up communtations/Comnand Fost in basement, irfe Commind Post,

Eattalion 2 supervised robisy stagdng Area,

Battalions 4, 6, and 7 operated on various floors gith unles so assigned.

Wiviston 3 operated as operations oTL on yax.oue floors.

Ditision 1 aperated at the comand post as cre

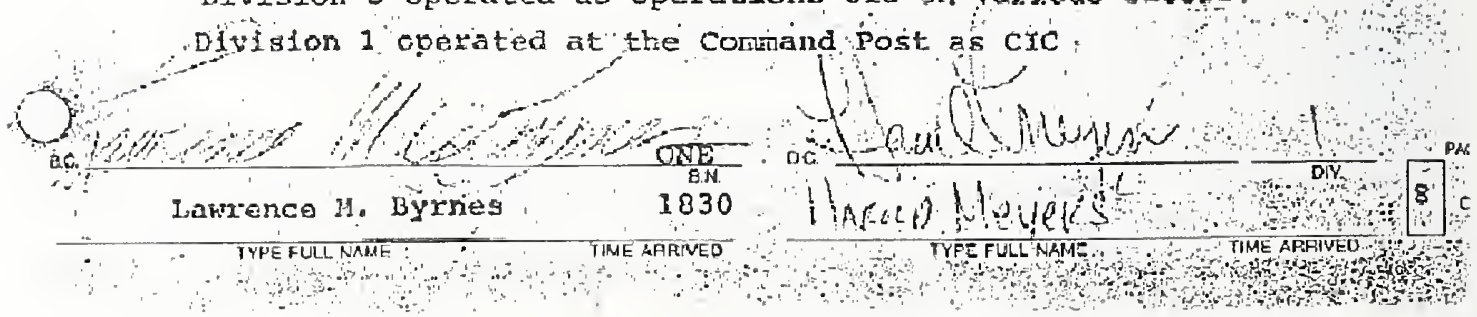




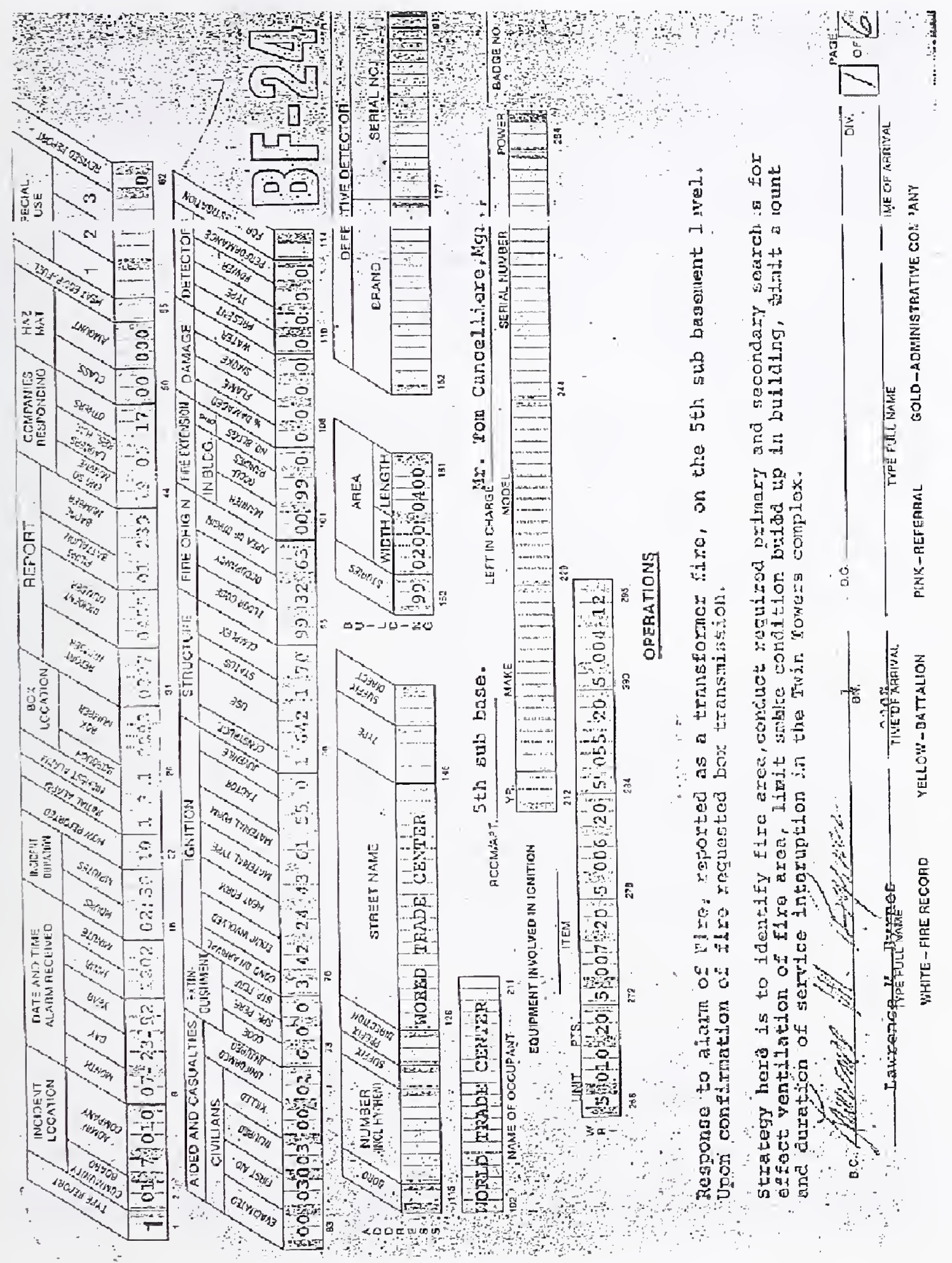




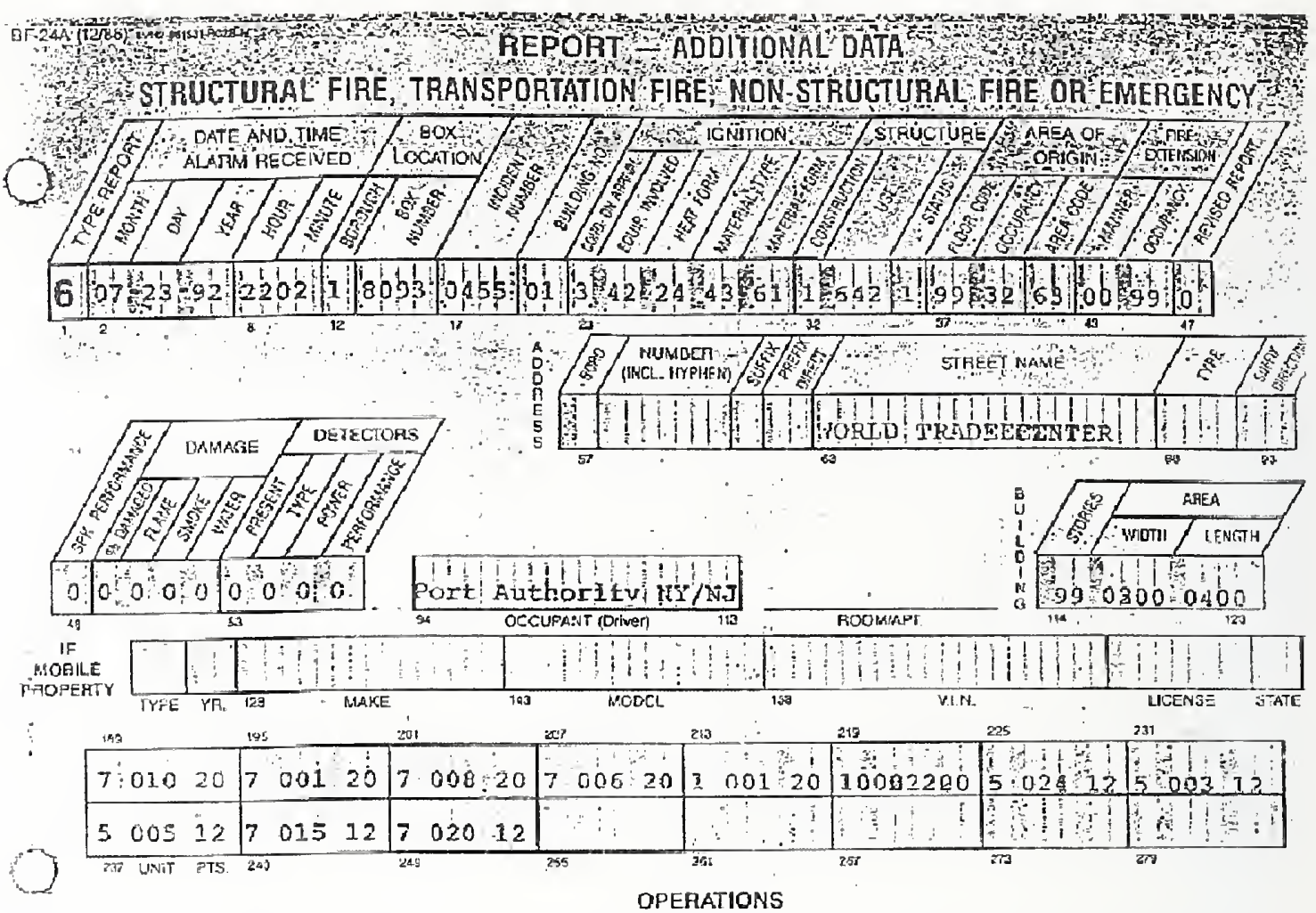

Rue to high electrical voltage 113000 ivolts confinged, no watez was used in

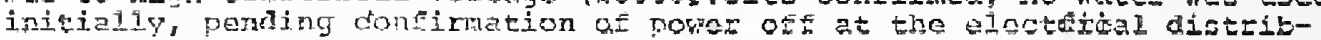
ution parcl.

Due to J,arge floor avea of the $5 \mathrm{th}$ floor-sub-inasenent, responding wilts vere split into feams vir: takder Co. 10 and Engine 10 using the K13 stair to aproach the fire adra, Jadicr 1 to use frecore staireay-K12, to access thy 5 th suls. basenent level. These units ware takigd with pinpointing the fire azed, an area of $200^{\prime}$ 's $400^{\prime}$.

A nocher of Ladder Co. I having round the fire situation in a very large poner ditrtibution panel, attonpicd to relay infoumation to his officer. Prior to his transtission firerjoghter was struck by a shock blist generated bye the involved parcl. Ladder 1 fircfighter krocked unconscienee required a conserted effort to remove to a separabe safe area.

Unit ODerations.

Engine 10 - Operated on 5th sub level, stretched a $22_{2}^{\text {t }}$ hand line Ermom the stapdipe, opersted when poiser off confimation received. Corpany

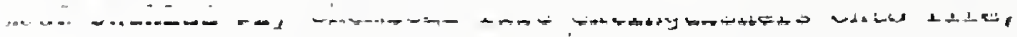

Engine 7 - operated on fire licor with line off standpipe, opergted

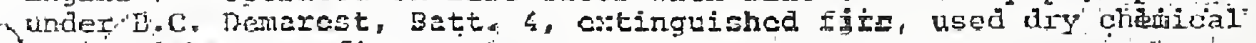
Jextinguishers un gire,

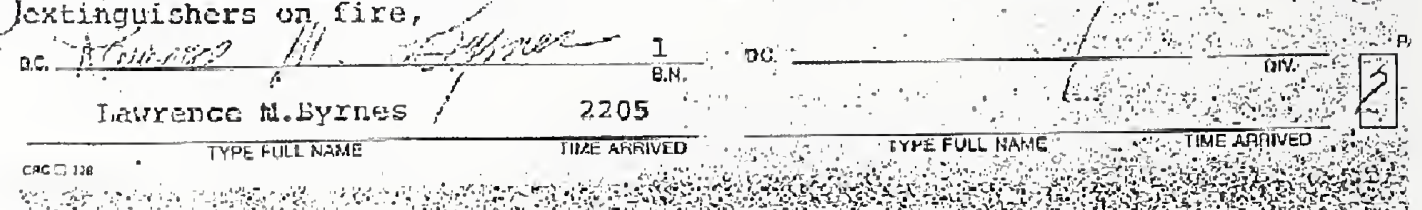




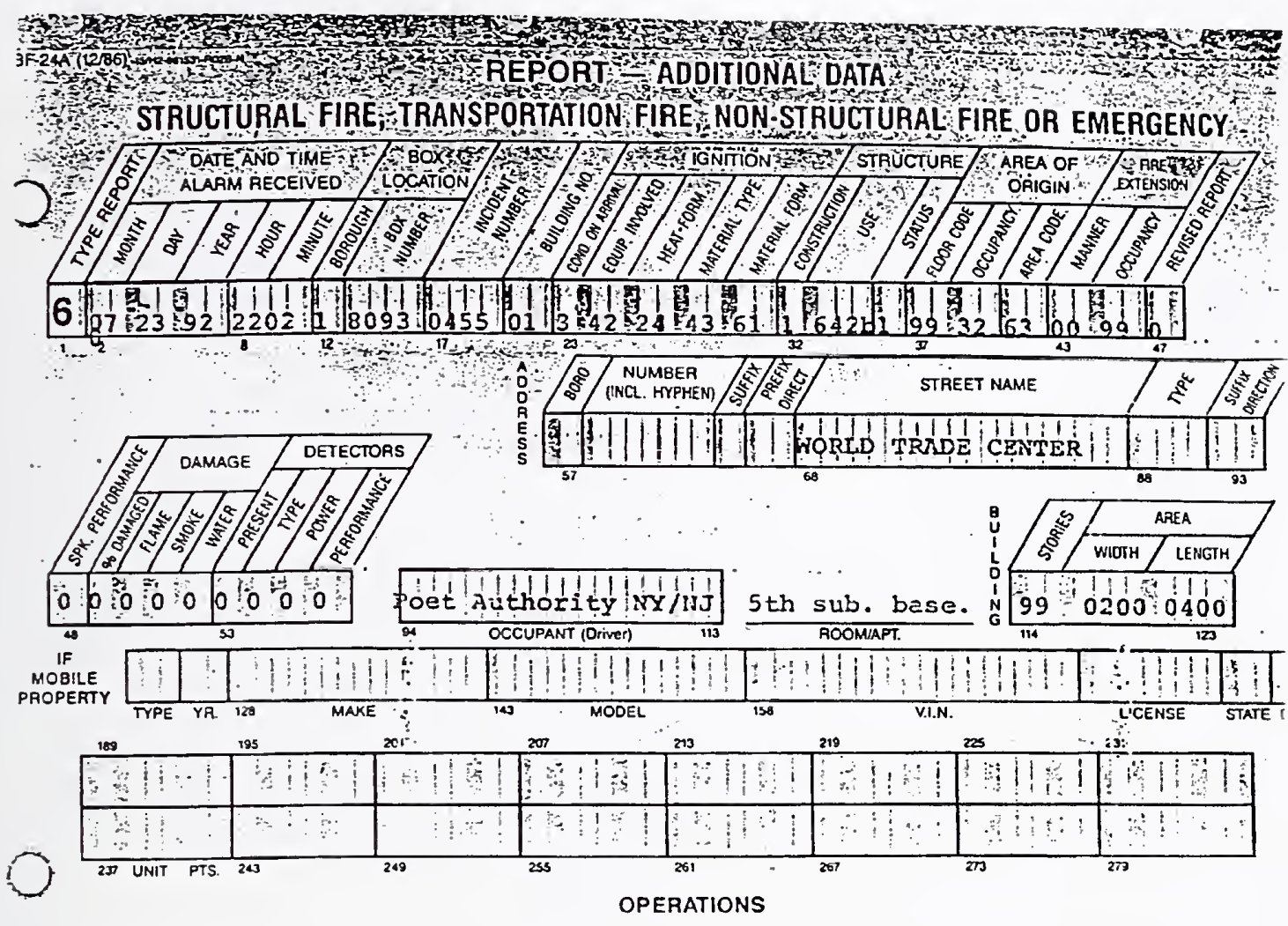

Operation cont:

Ëngine 6 - Assisted Eng 7 in stretch of and operation of a $2^{1}{ }^{\prime \prime}$ line into fire area, performed search of area, overlauled as neceesary,.

Engine 55 - Operated with and relieved Eng. 7 on hand line on fire floor, took up hose lines,

Engine 4-Under supervision of BC. Turnee,B2, transported injured member of Lad. I to ambulance on the BI level of the fire building,relieved Eng. 10 on a Hand line, oberhauled, took up hand iine.

Englne 24 - Transported Air Cylingees fire area under supervision of BC. Jackson,

Engine 3 - Ordered to and did provide air cylingers to operatind units of the 35 level.

Engin

Stood Fast - Eng9Satl, Engines 15, 23. 33, 34, 207/Maxi, 284/Sat.3,

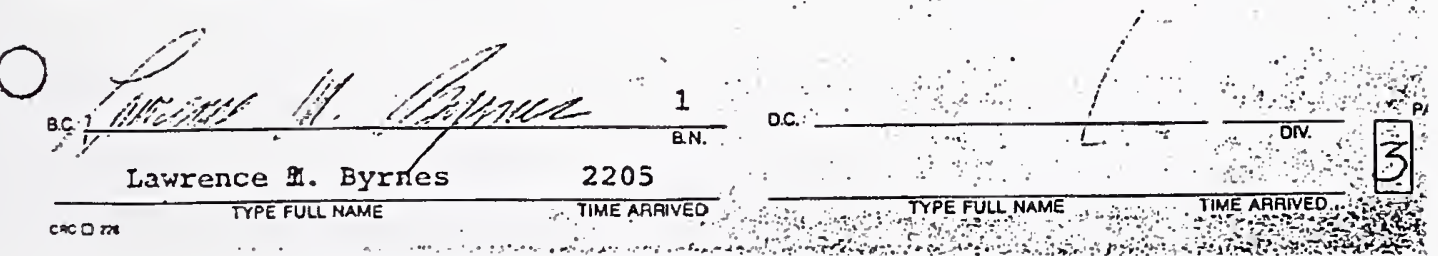




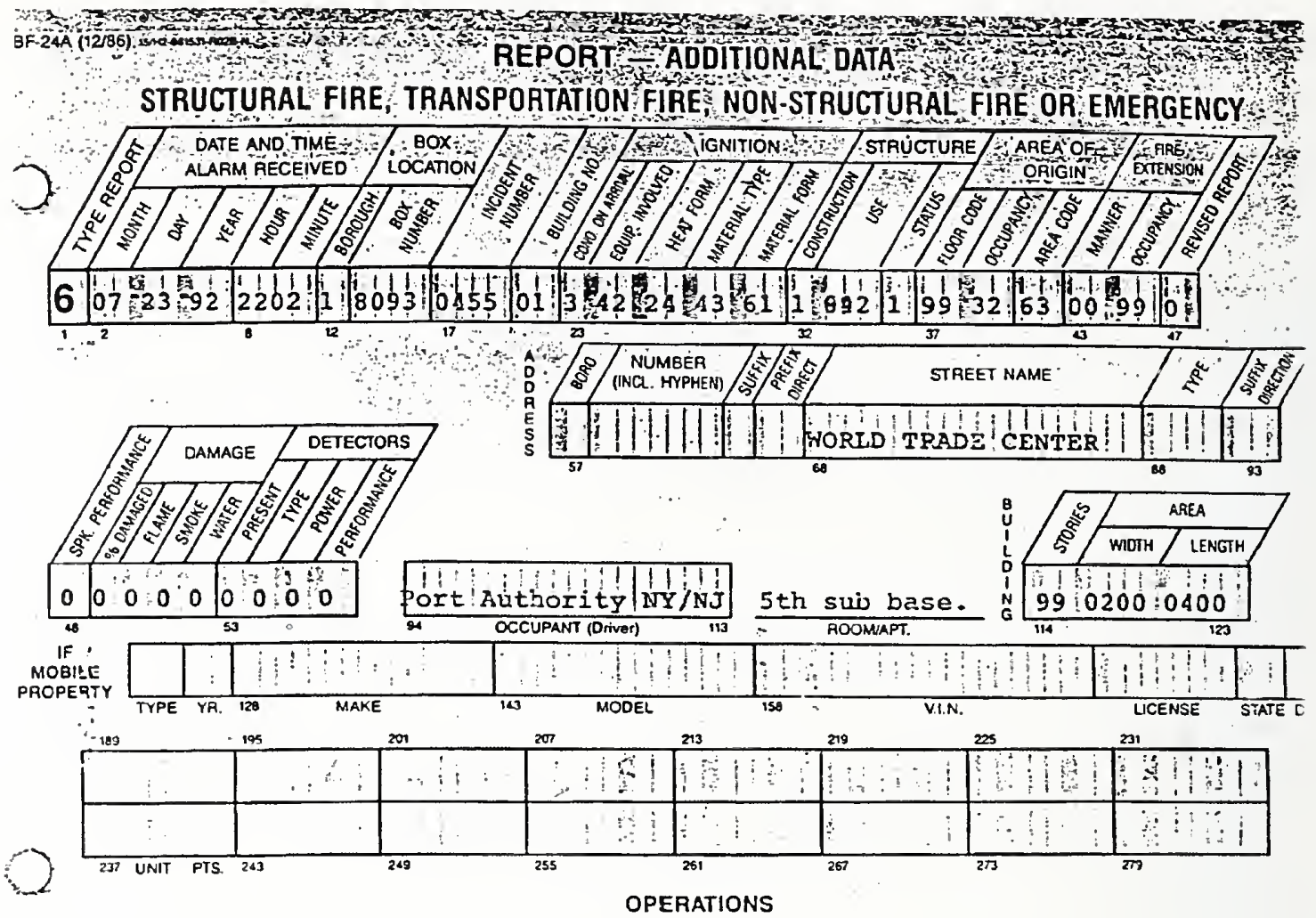

operations cont:

jecider 1- Dowated at the 35 level, conducted seadch to pinpoint the fire area, concucted a primary search for nossible employees trapped. FF'. Amodio injured in explosion of 13000 volt distribution panel,

Ladder 10- Performed a scarch of the 55 level lito identify the fire area, and searched for possible trapped employees, gathered and used lry cl:imical extinguishers on the fire prior to pover removal, onerhauled as reguired,

iadder $\varepsilon-$ Performad a secondary search of the fire area, used dry chenical axtinguishers, assisted in overhauling,

Laddrr 6- Pajced and used portable exhaust fans in stairgells to effłcit ventilation, took up,

Tradier 15- Sunolied snare SCBA crlinders to stanina irea,

Ladder 20- Supplied spare SCBA cylinders to staging area,

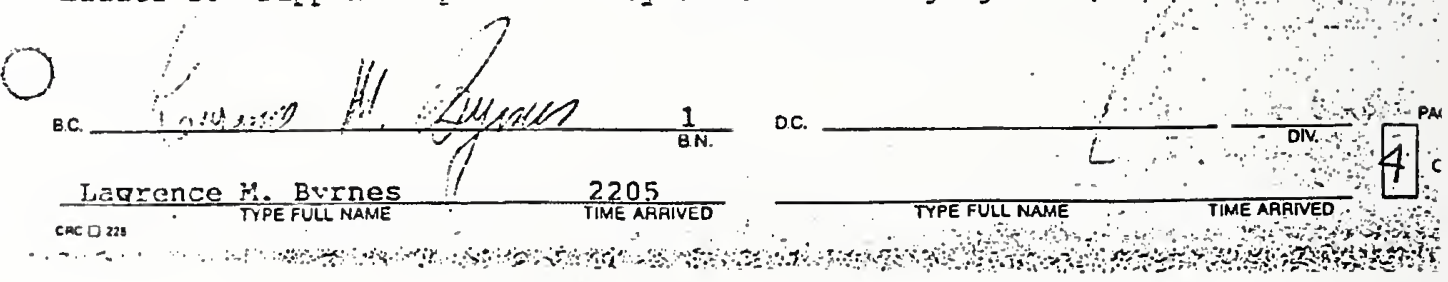




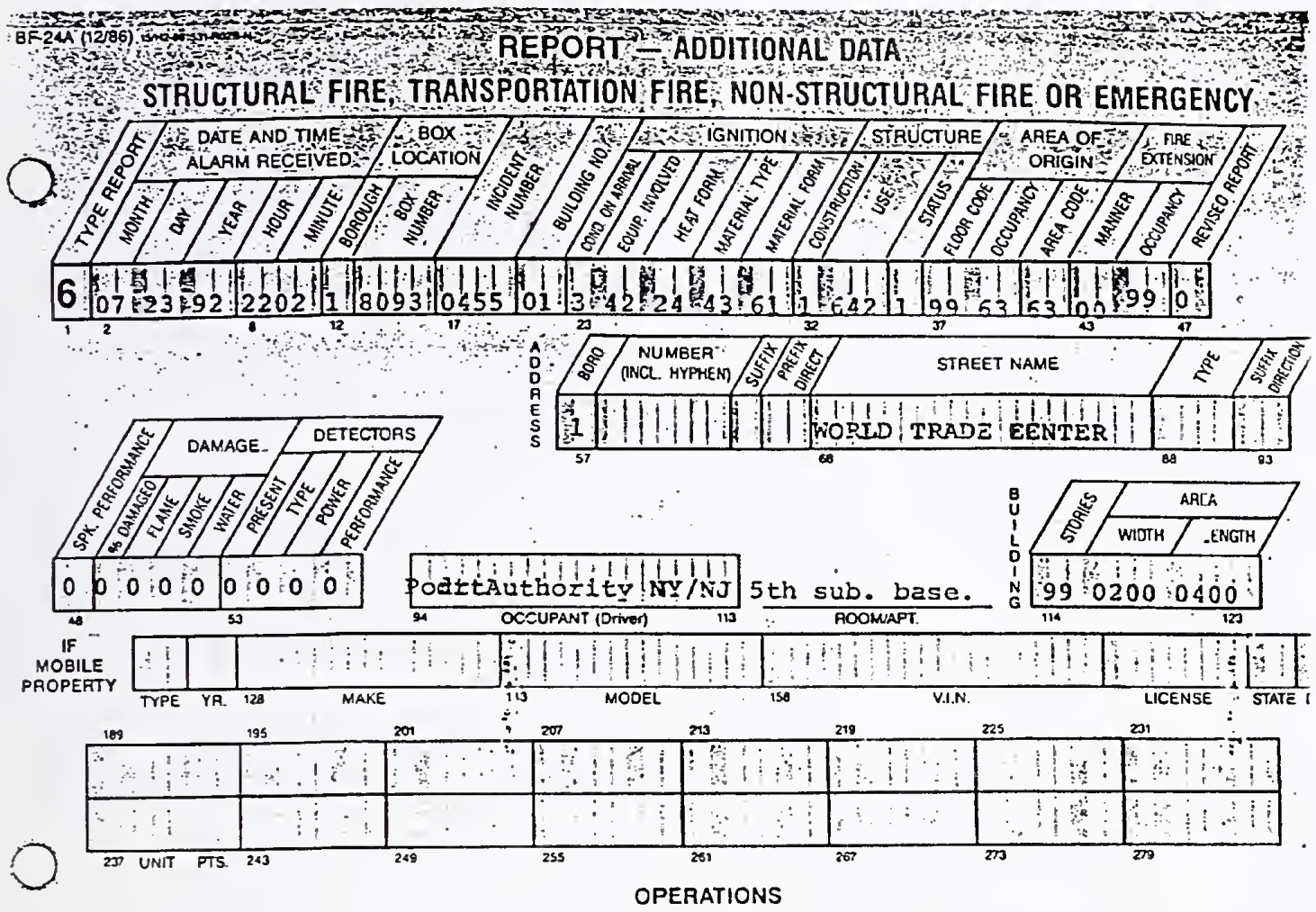

Operation cont:

Rebsue i- Company split to perform several íperations-

1. Assisted in administering first aid to Injured firefighter,

2. Conducted seconáary search of Fire arca, liegative,

3. Used Thermal Camera to chec!: Lor tossible fire extension,

Rescue 2- Assisted (2) civilian electricians (with SCBA's) to confirm power off in electricalpanel, relayed confination of power off to Comnand Post. Essisted in hand line iperation, assisted in VES of Eire floor.

Chief Officers present: D.AC R. Palmer, Cir Duty DC. R. Manson Div. 1 , B.C. I. Byrnes Batt. I, B.C. i.Demarest Batt. 4, 3.C. R.Turner, Batt. 2, B.C. Costa Batt. 7,
B.C. Miccio Batt. 6; B.C. iJardone Batt. 9.

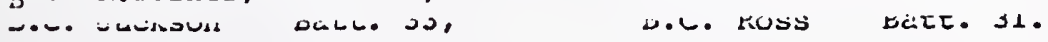

().

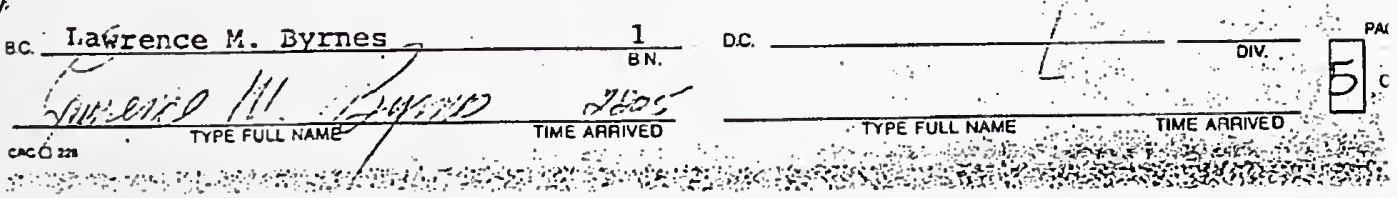




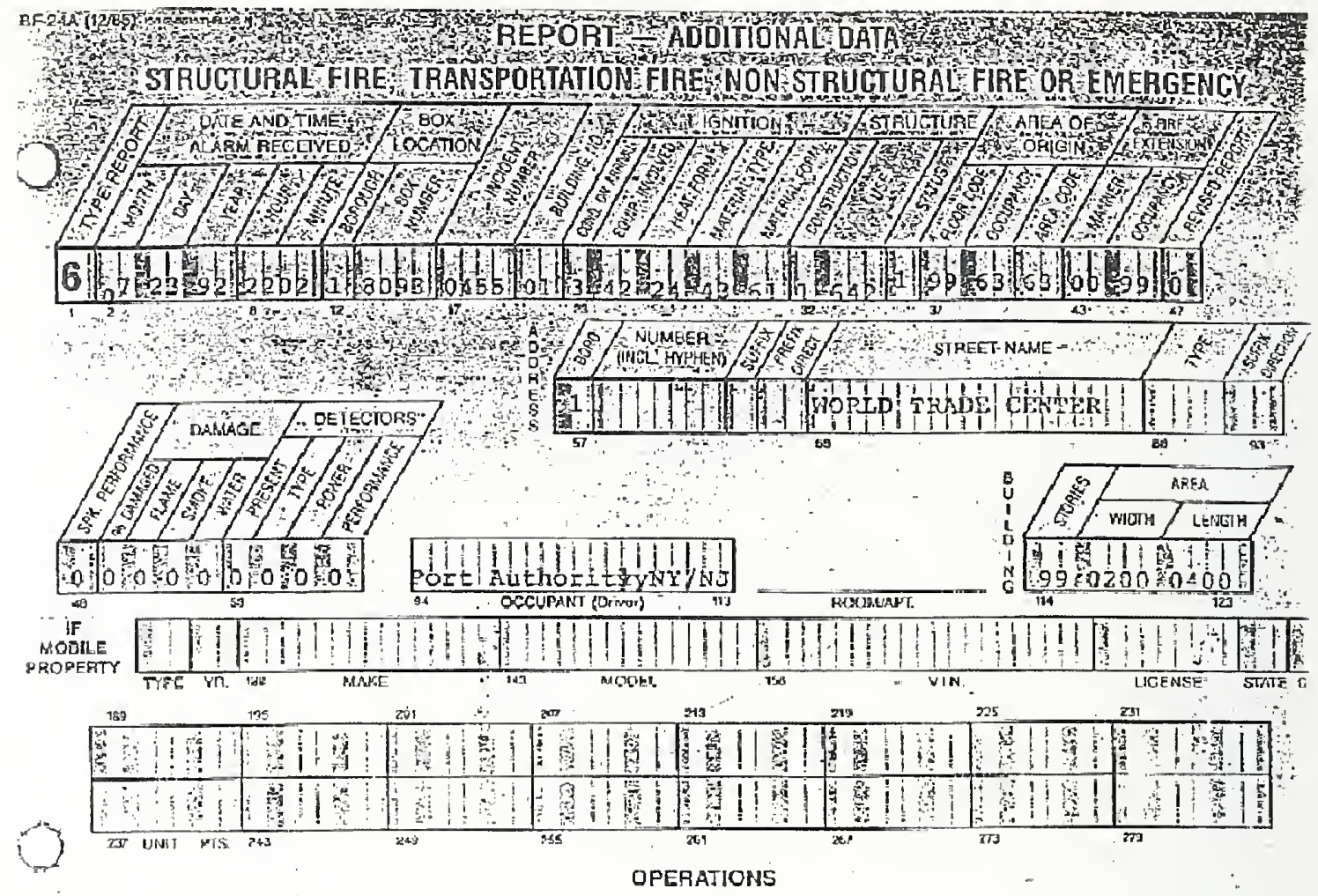

Dpersitions coit:

Grocter alarms transinjted dur to شagnituce of fire area involved, potential EOI smose and heat probleras, numbers of enployees in building need rosurply and relicvo operating porsonne.

Injuries: FF. Mmodio, Lad. 1, concussion, remolu to Eecknan Hosp., FE. Cancel, Lad 10, granited 1 I.

FF. Hanson, Iad.10, granted M.., FW, Selletí, lad. 10, Granted it.

Note: Staff and aployees of the New Yoric Port Nuthority proviced excellent guidance and assistance. lielp provideg included mechanical, ventilation, electrical aistaibueion disciplines which rusle for a ruch eesier fire ground operationg

h.

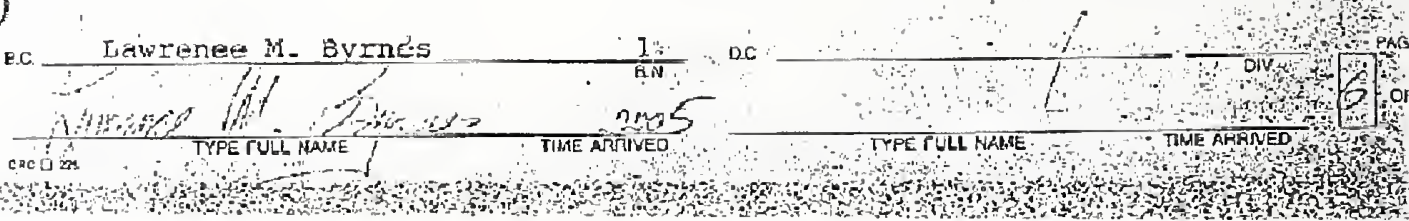




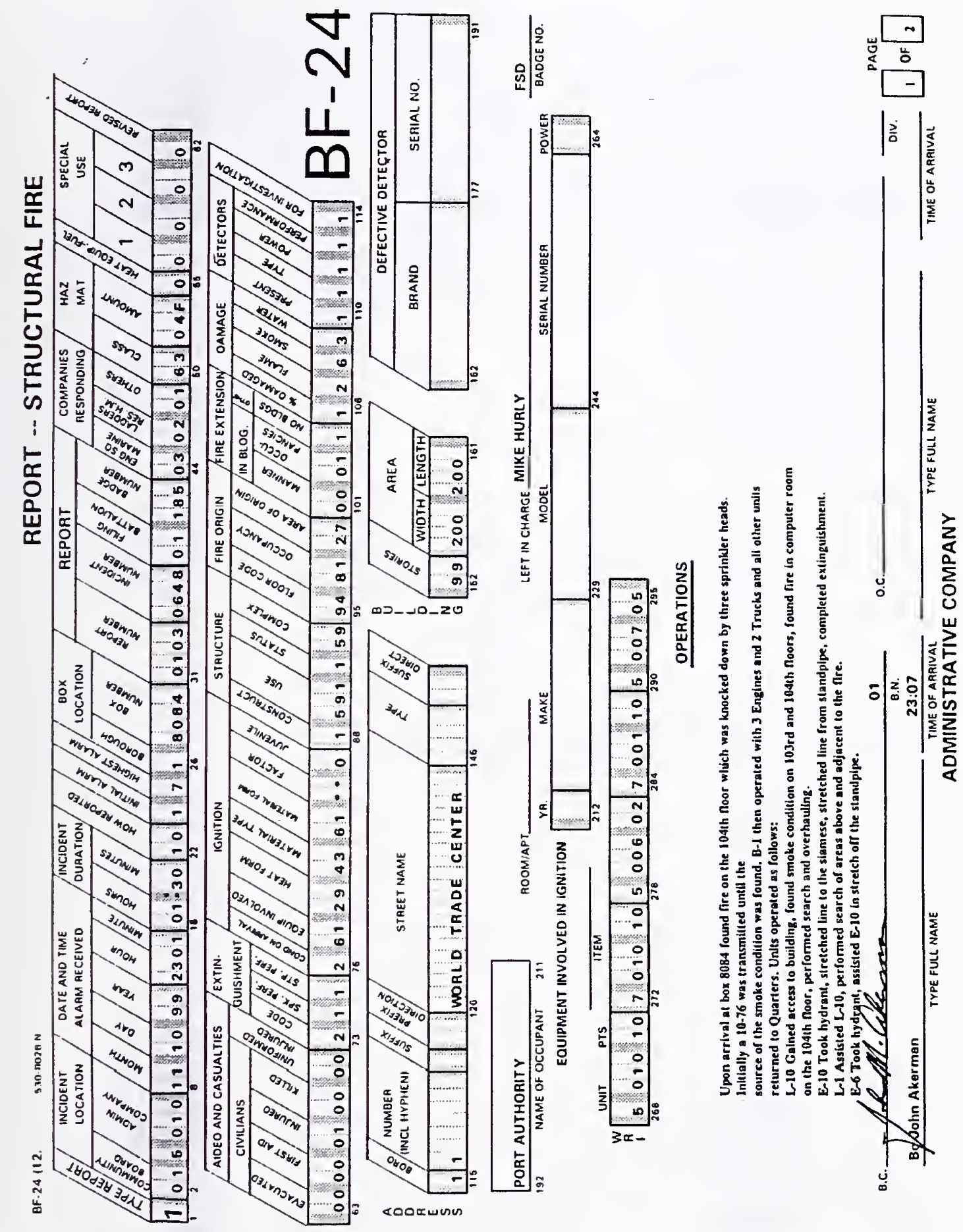


F-24A (12/86) IBNe Hols3inozs en

REPORT -- ADDITIONAL DATA

STRUCTURAL FIRE, TRANSPORTATION FIRE, NON-STRUCTURAL FIRE OR EMERGENCY
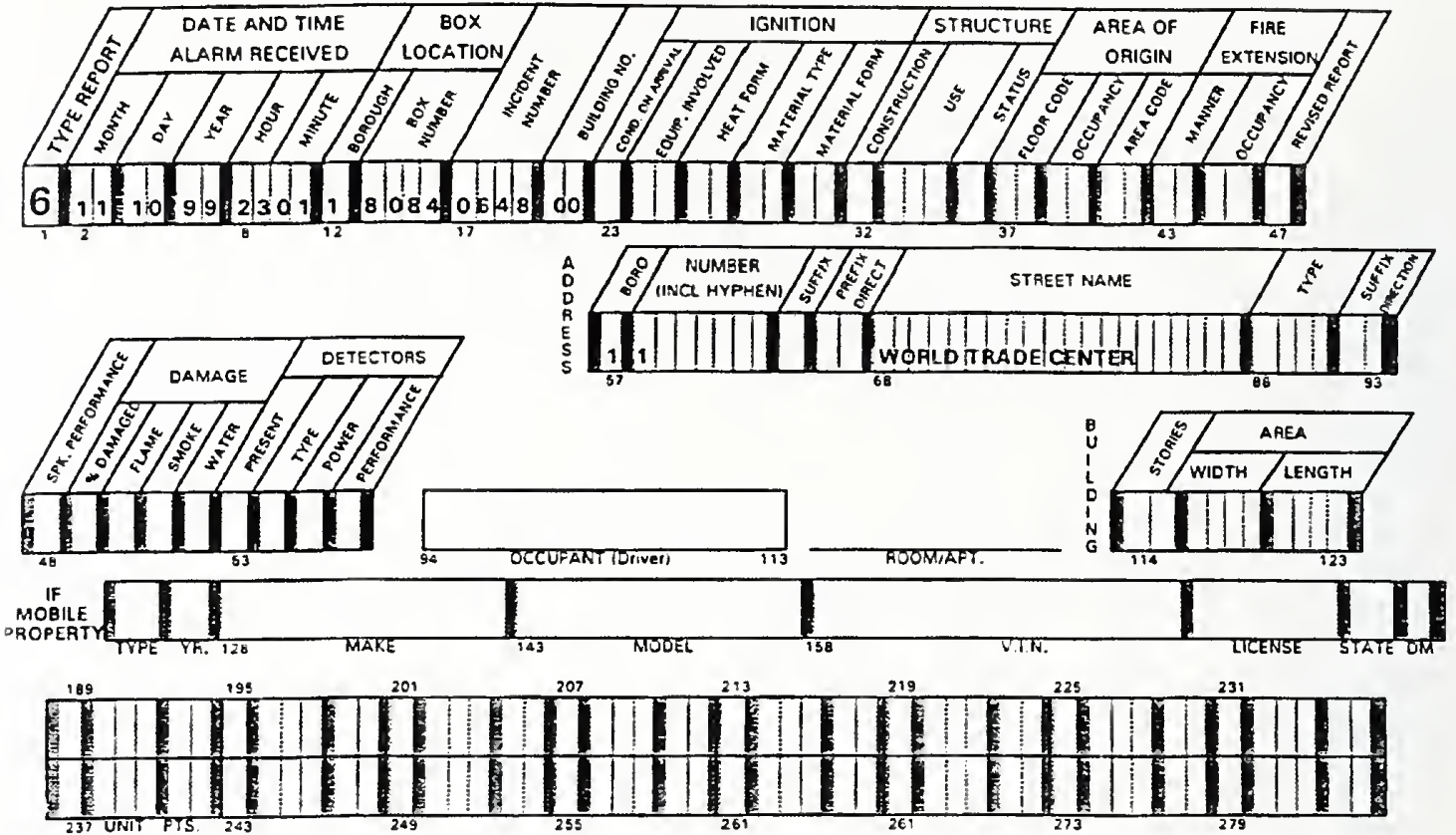

OPERATIONS

E-7 Stretched precaurionary line from standpipe.

F.S.D. for World Trade Ceater - Mr. Mike Hurly.

O.E.M. Lt. Wilson.

F.M. Kregler 1361 responded on BFT's own bnowledge of prior suspicious activity. Job $\$ 11201$.
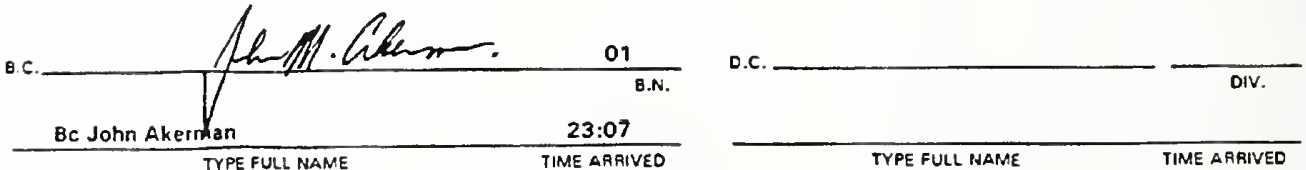

2 PAGE 


\section{Attachment G-A.5}

Significant fire incidents occurring in WTC 2 (3)

\begin{tabular}{|c|c|c|c|c|c|c|}
\hline $\begin{array}{c}\text { Significant } \\
\text { Fire }\end{array}$ & $\begin{array}{c}\text { Incident } \\
\text { Date }\end{array}$ & Fire Location & $\begin{array}{c}\text { \# Sprinklers } \\
\text { Activated }\end{array}$ & $\begin{array}{c}\text { \# Standpipes } \\
\text { Activated }\end{array}$ & $\begin{array}{c}\text { Cause of } \\
\text { Fire }\end{array}$ & $\begin{array}{c}\text { Material } \\
\text { Ignited }\end{array}$ \\
\hline 1 & $5 / 19 / 75$ & Floor 32 & - & 3 & Incendiary & Trash/waste \\
\hline 2 & $4 / 12 / 77$ & $\begin{array}{c}\text { Duct work over } \\
\text { grill in restaurant } \\
\text { on floor 107 }\end{array}$ & 2 & & $\begin{array}{c}\text { None } \\
\text { listed }\end{array}$ & Duct work \\
\hline 3 & $3 / 22 / 93$ & $\begin{array}{c}\text { Fan motor room } \\
\text { on floor 108 }\end{array}$ & 2 & & $\begin{array}{c}\text { Mechanic } \\
\text { al failure }\end{array}$ & $\begin{array}{c}\text { Not } \\
\text { classified }\end{array}$ \\
\hline
\end{tabular}




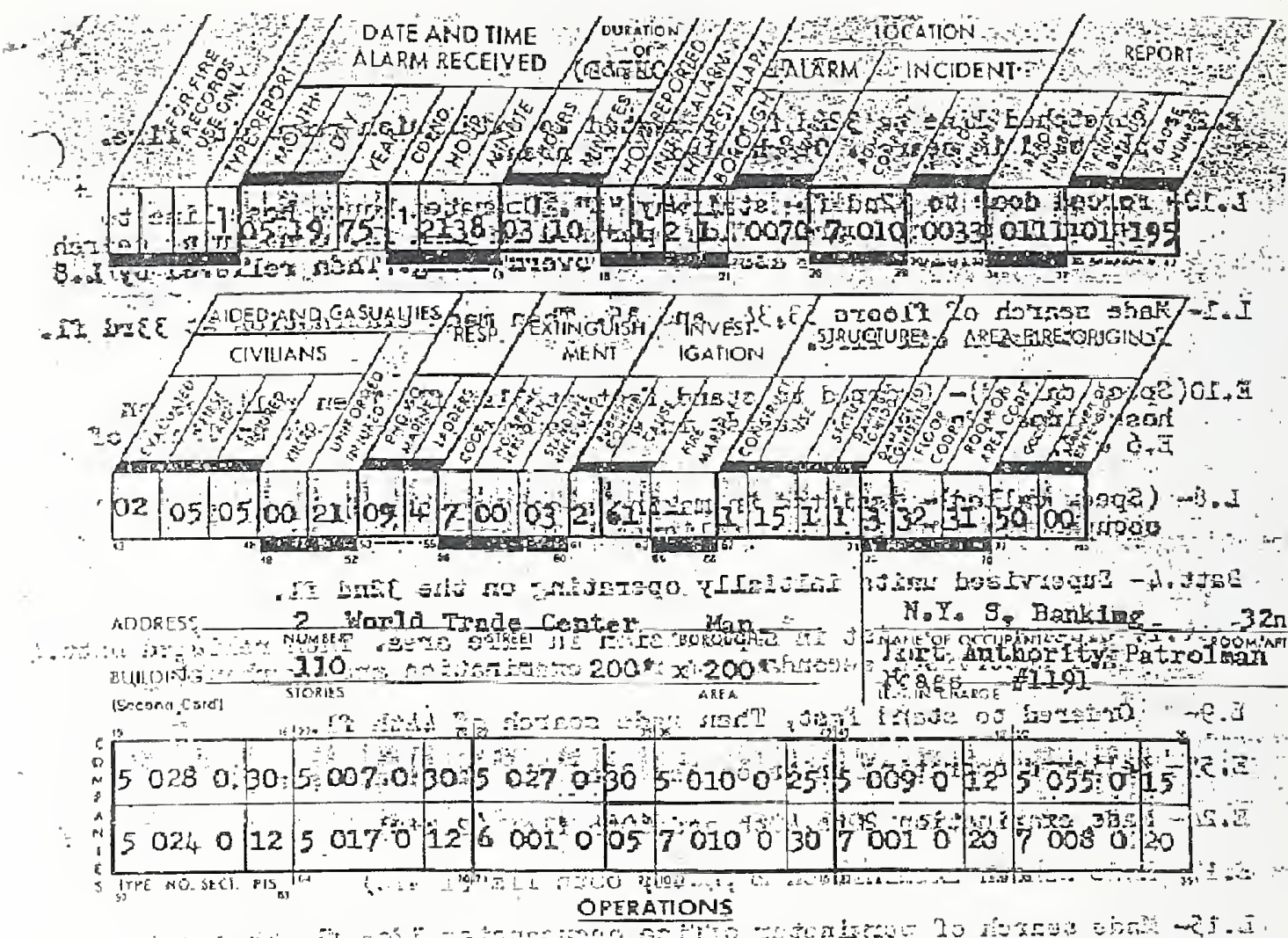

Ipon ars

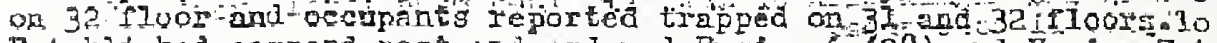
Established comand post and ordared Engine $b$ (28) and Engine 7 to groeees

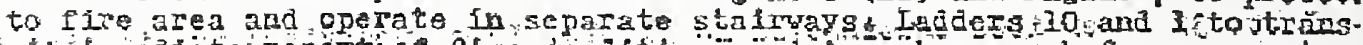

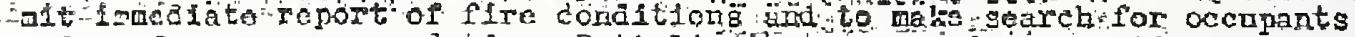
and perform necosstry dutieg. Rattalion to supervise operations in fire

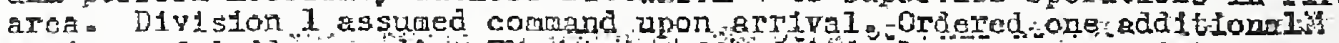

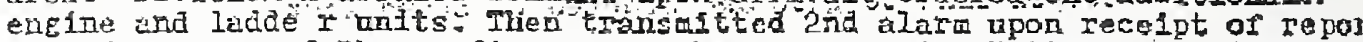

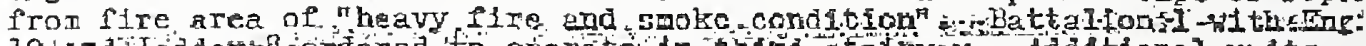

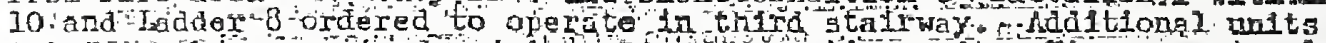
pla to core area throughout and was confinad to samo.

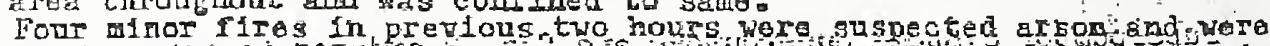

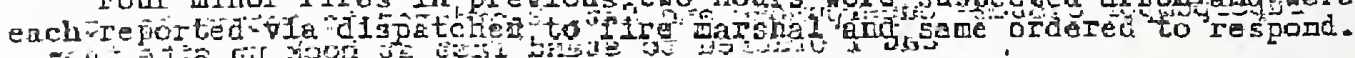
E. $6(20)$ Stretahed Ifac to $32 \mathrm{fl}$ 住

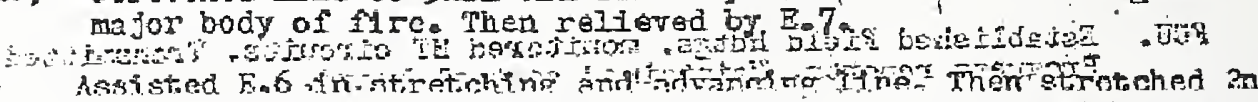

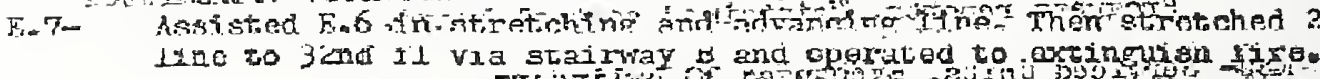
a

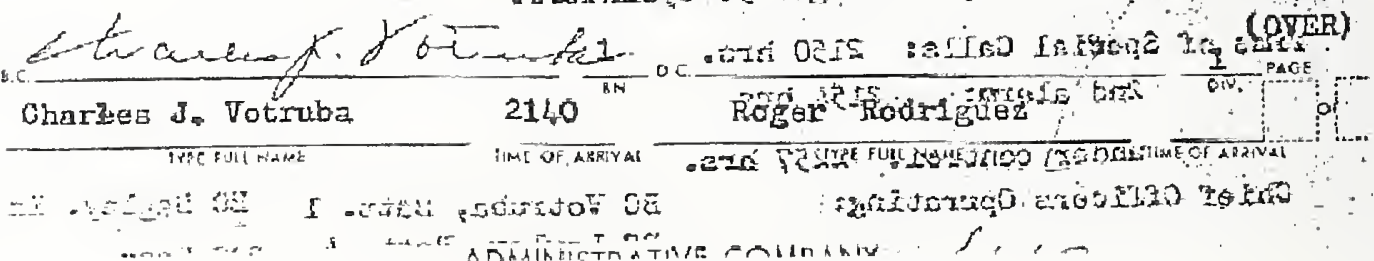




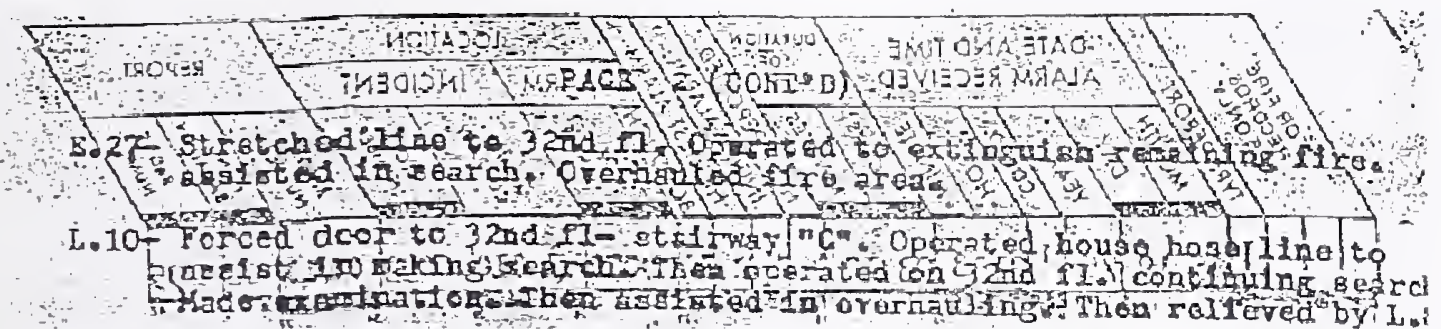

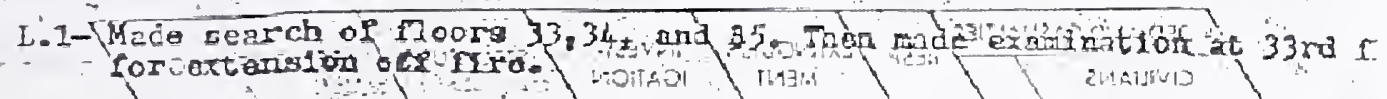

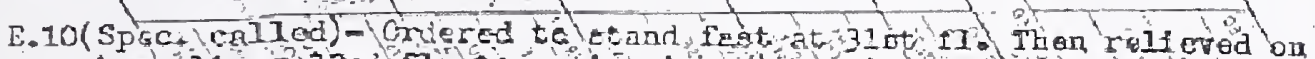

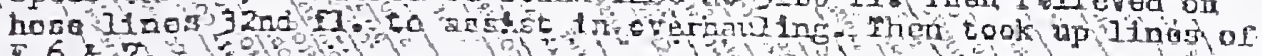

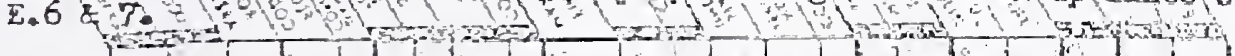

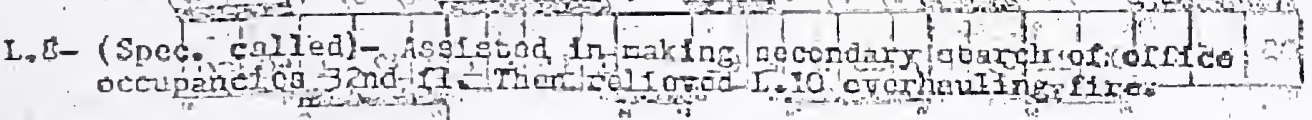

Brtt.4- Supervised untu fratraly operating on the 32nd fl.

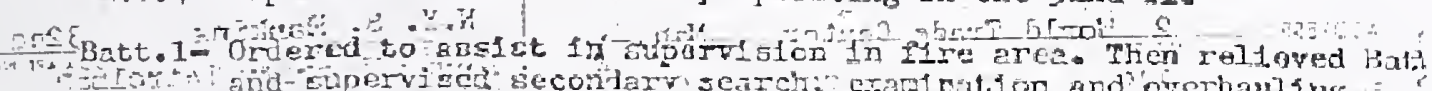

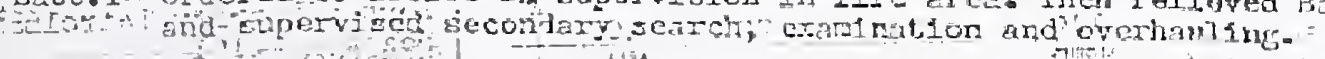

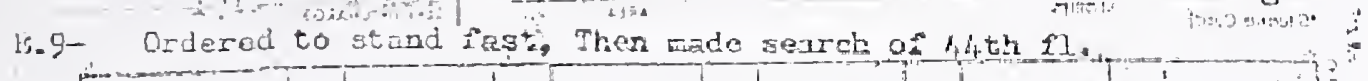

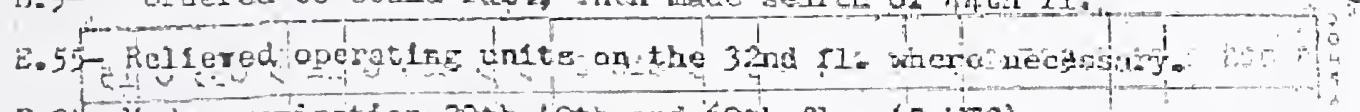

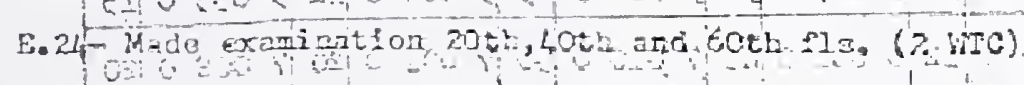

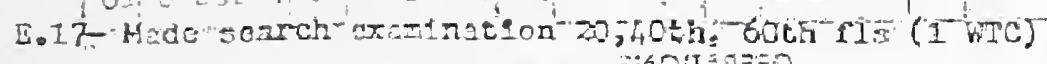

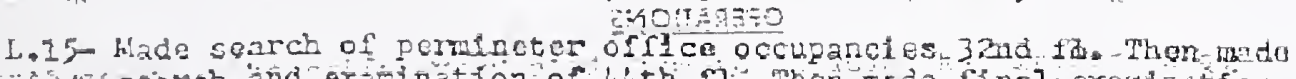

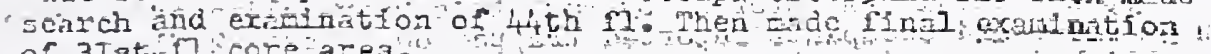
or. 3Istim core area-"

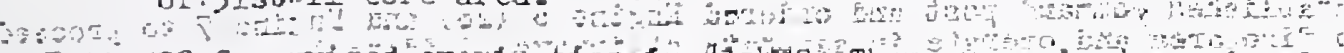

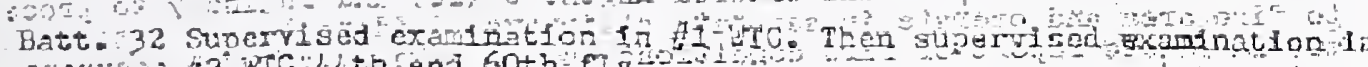

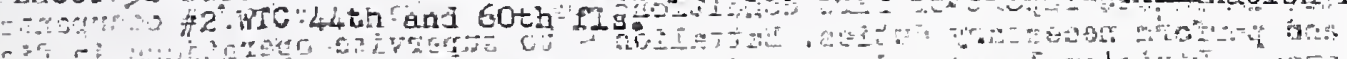

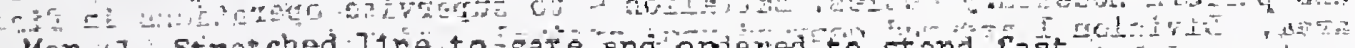

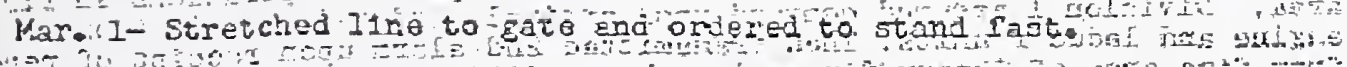

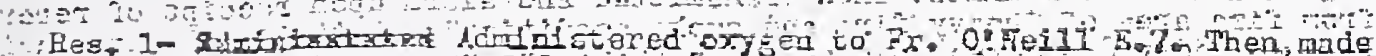

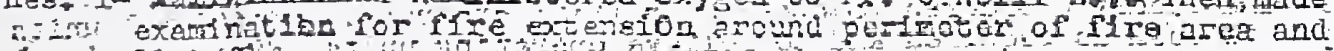

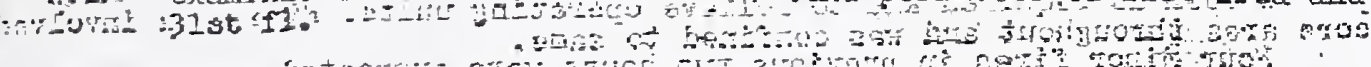

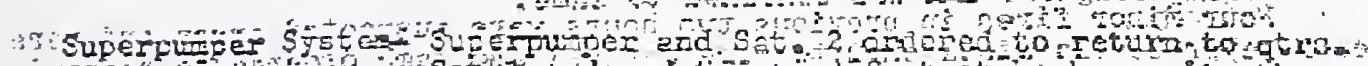
-

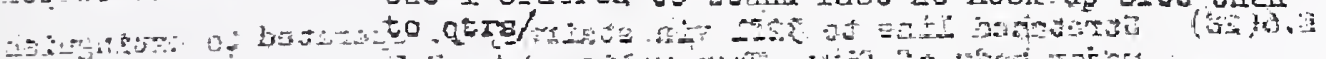

PCD atoblished Field

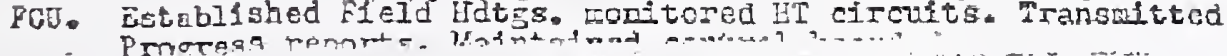

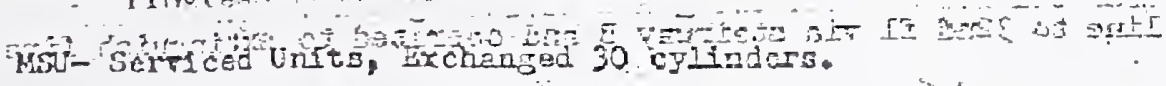

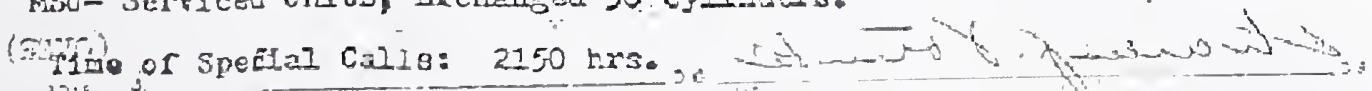

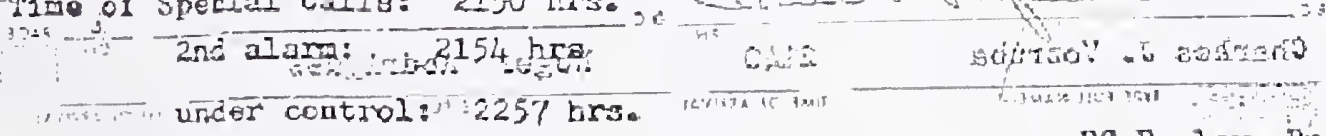

Chier orficars Operating:

BC Totmiba, Batt. I BC Barloy, Bn 


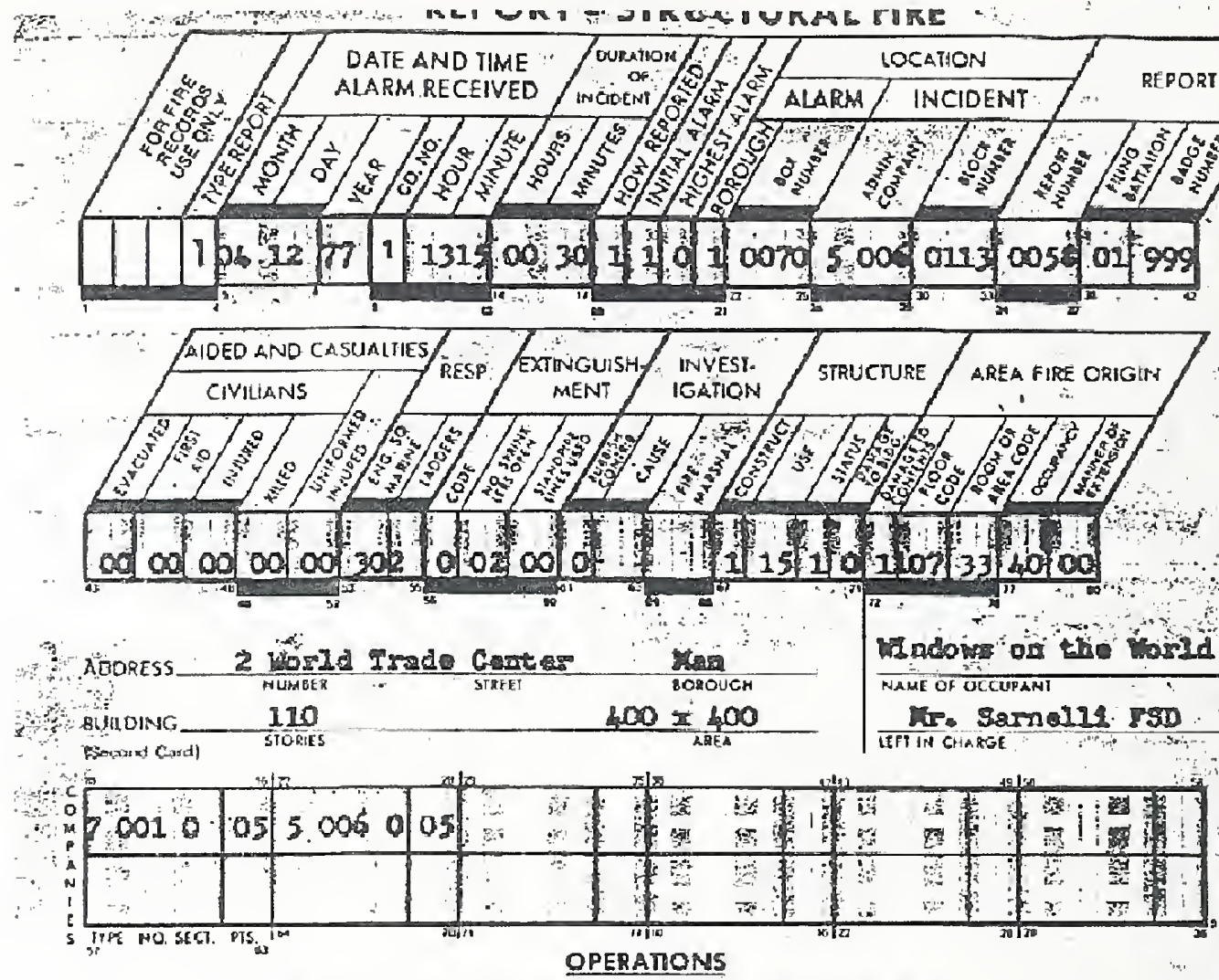

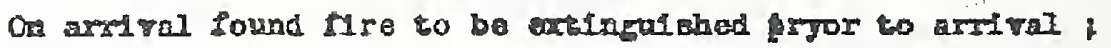

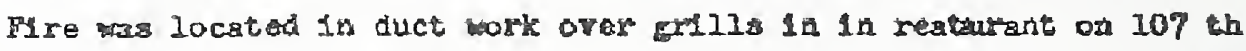

1200. Th. 1 notsfled depatcher to notlfy board of Health of posable

food contandation from beat omoke and gasis in gestaurant.

B. 6 Tooir rolled ups to 107 floox stretched Ilne and stood faEt.

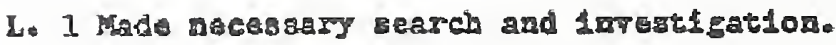

F. 2 od ucene replaced two aprinkler hejds.

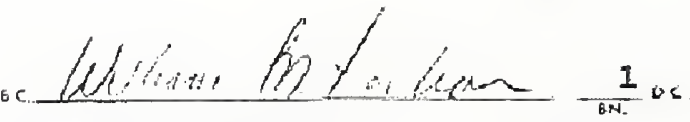

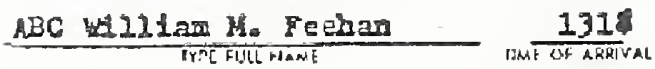




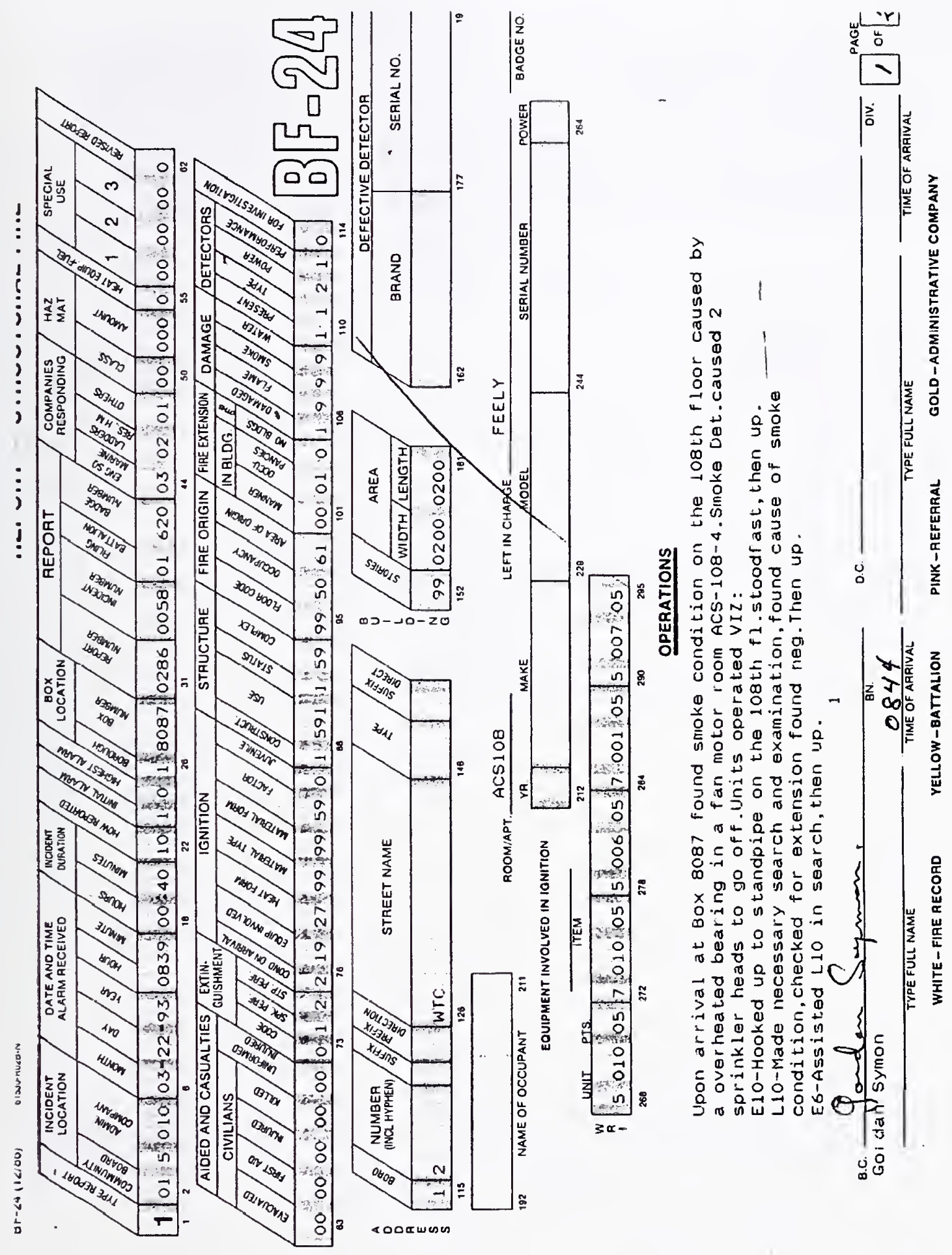




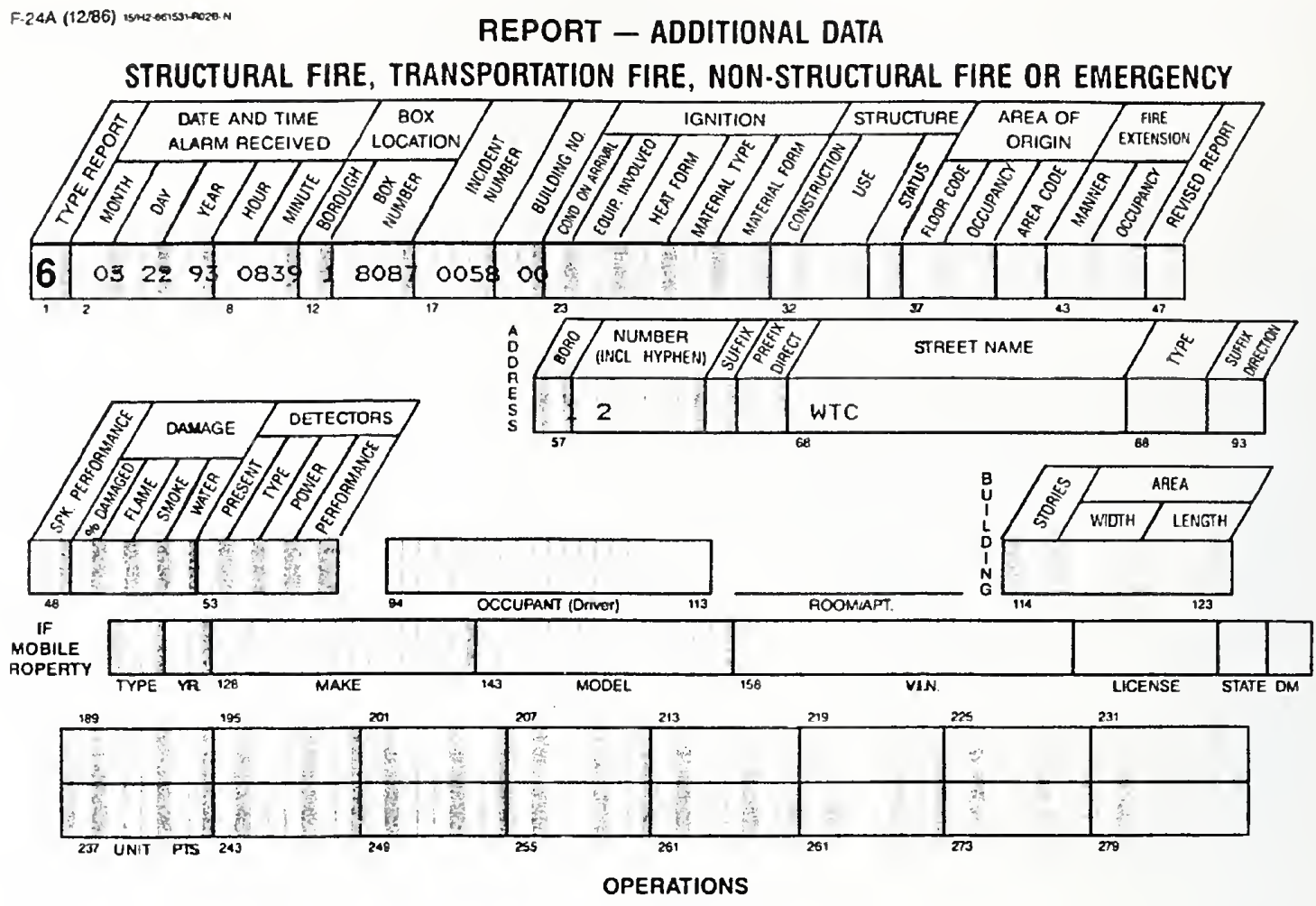

E7-Assisted ElO in stretching 1 ine, then up.

L1-Assisted L10 in search, then up.

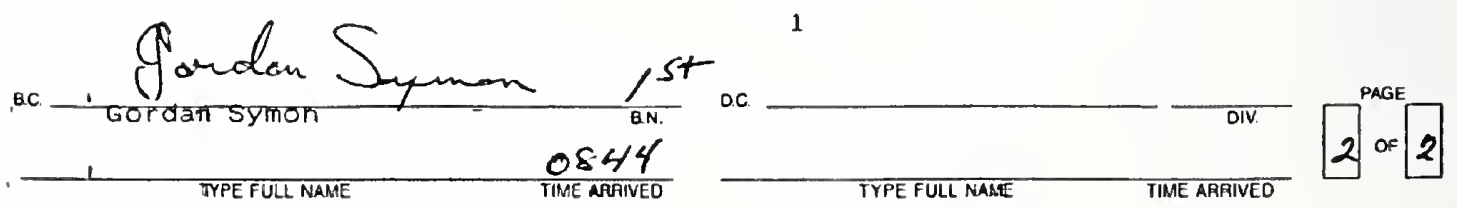




\section{Attachment G-A.6.1}

Additional fire incidents involving the deplpyment of standpipe lines in WTC 1 and WTC 2 -

- Fires involving the use of one standpipe line and the activation of one sprinkler (4 in total) 


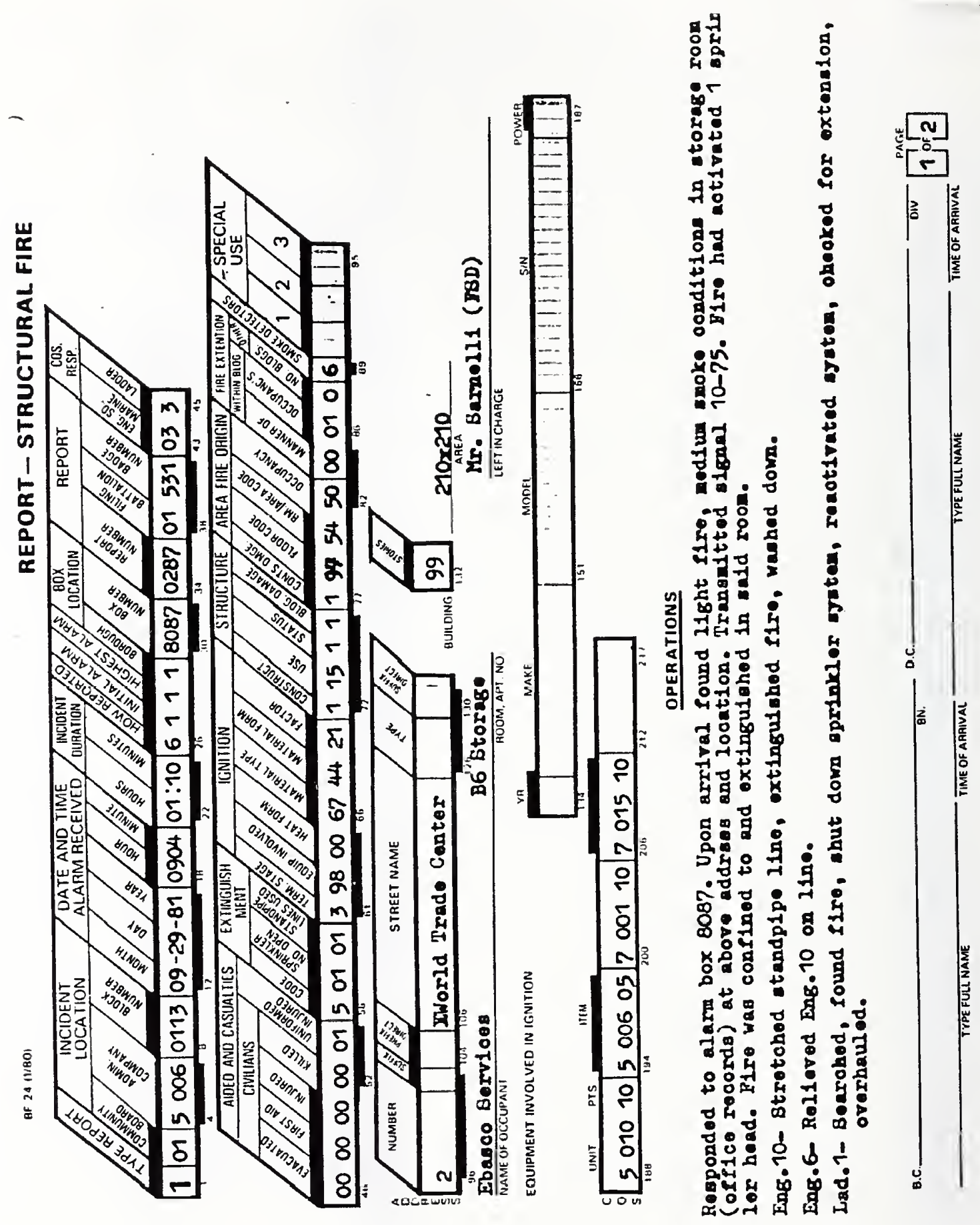

A.DMINISTRATIVE COMPANY 


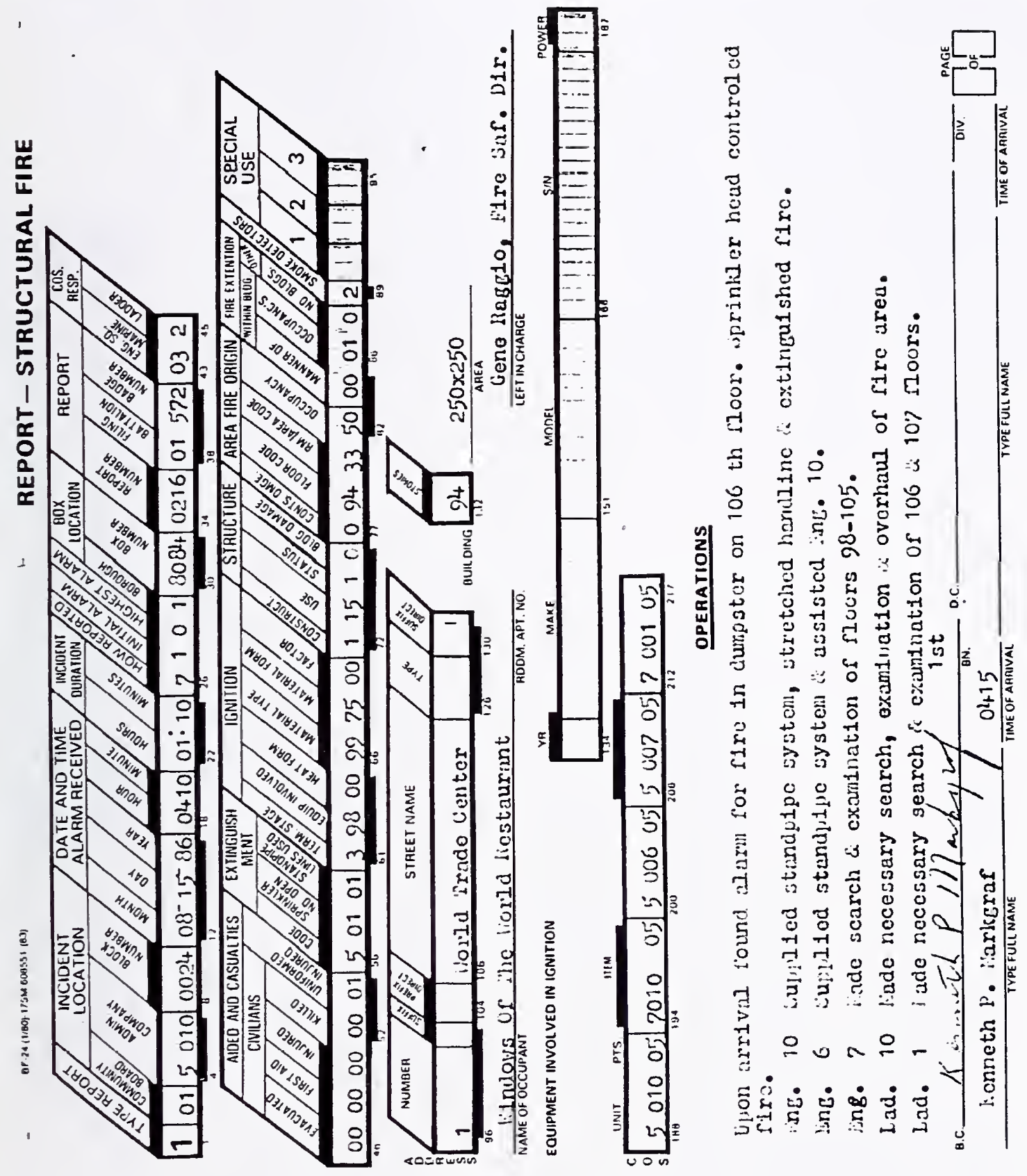




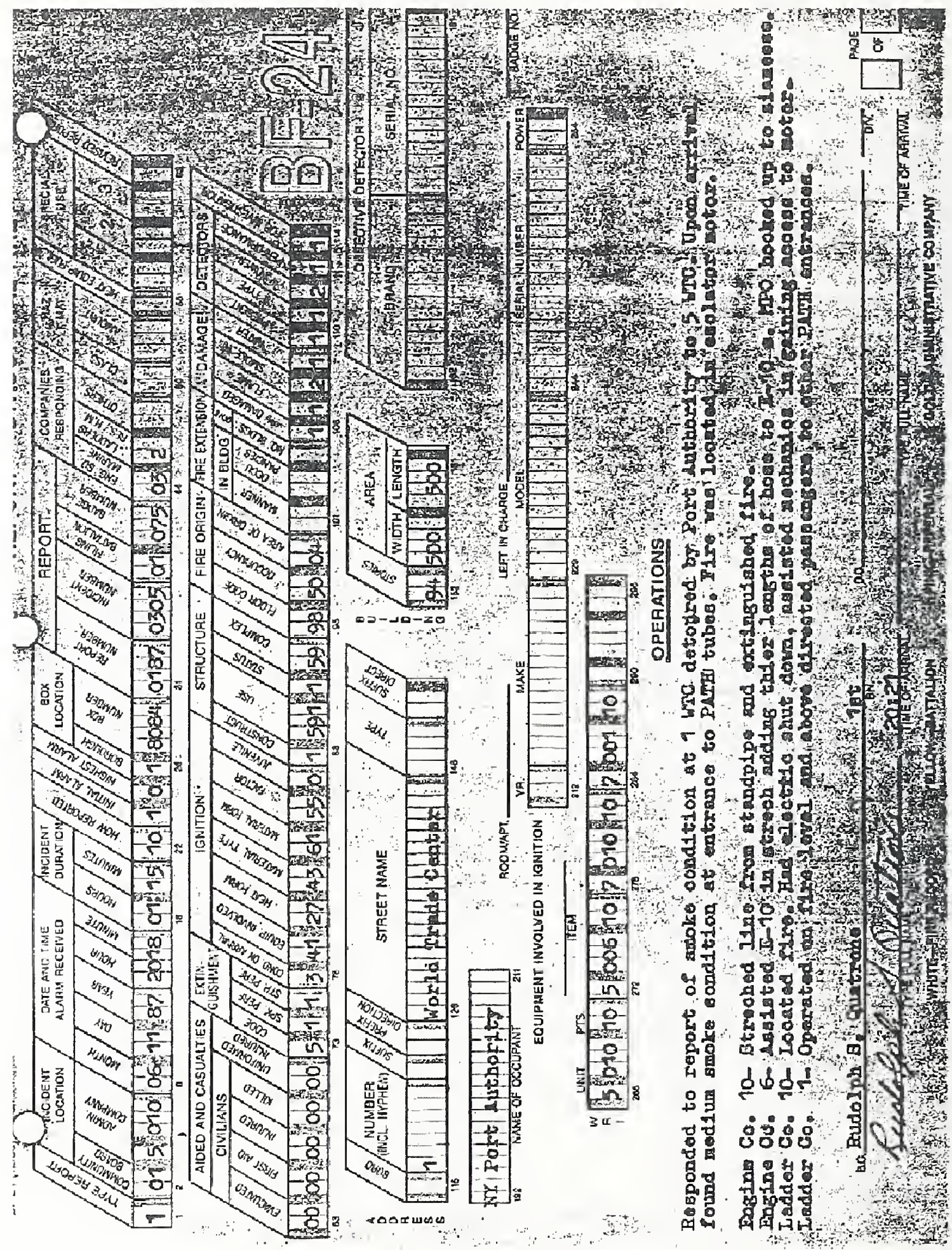




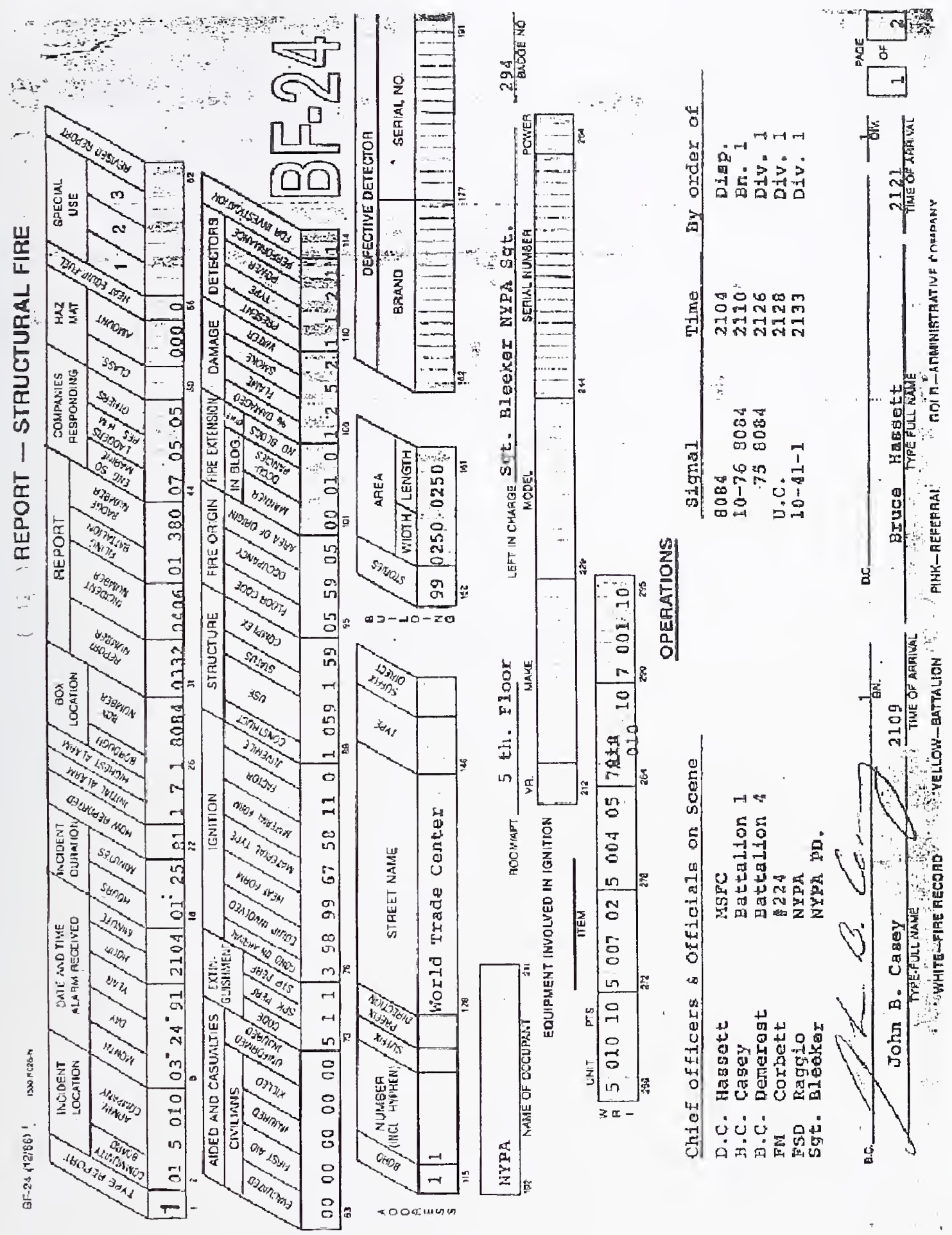


This page intentionally left blank 


\section{Attachment G-A.6.2}

Additional fire incidents involving the deployment of standpipe lines in WTC 1 and WTC 2 -

- Fires involving the use of one standpipe line (27 in total) 


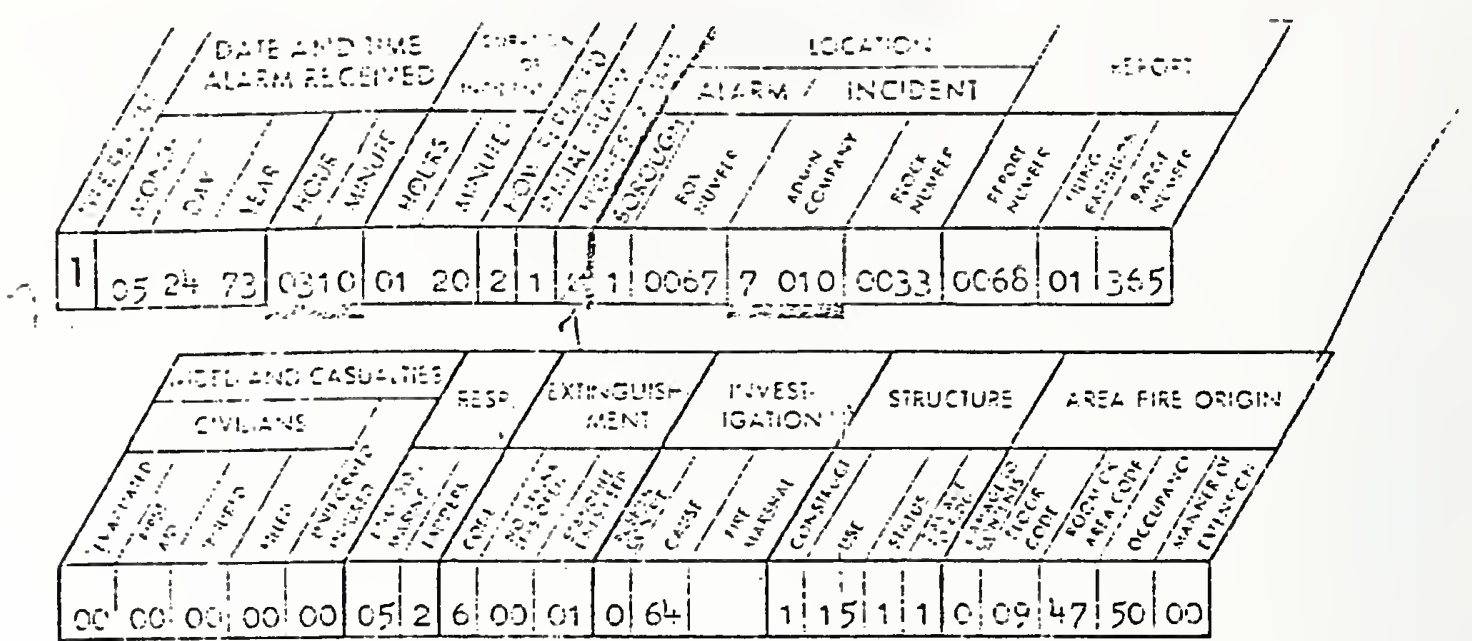

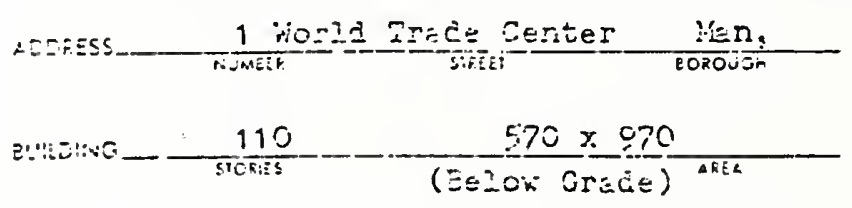
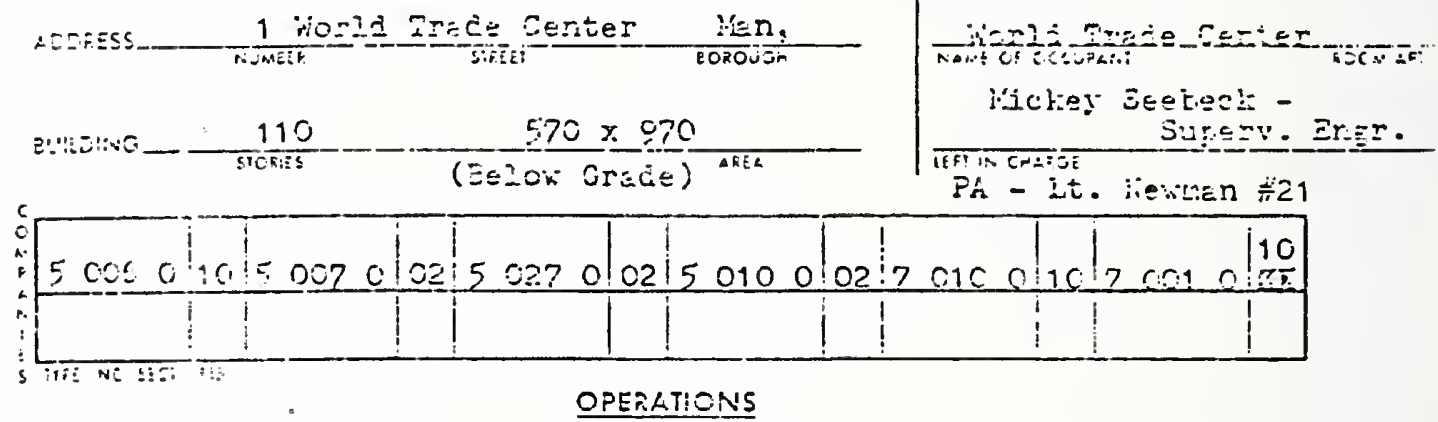

On errivel found fire in elevetor car (Jj) - B2 ievel, -J山 aree Ö:

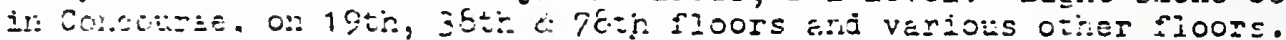

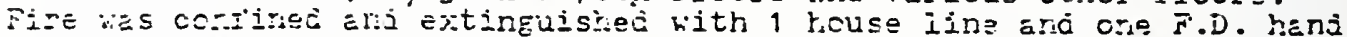

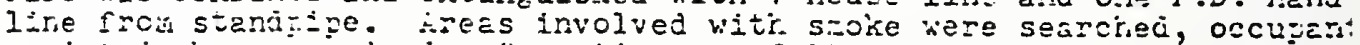
ensisted hitere rejuireci. Operctions as follov:s:

E6- streich iine from starcipife J-4 area, ext. fire in elev. \& sheft. ت7 - Essist $\Xi 6$ in stretching haziline.

E10 - Eese seerch upper fioors - 77 th to 79 th (lignt smoke cord.)

327 - Eade seercí upes flloors - 36th fl. E vic.

LiO(its) - Teport to CP - search \& Examination of concourse area.

Li - forcea telev. docr E-2 level, examire aar, overnaul.

$B 2$ - surervise operations of $E 6 \&$ Li opening eles. cer door \& ext. firt $i-2$ Ie $I \leqslant v e l\left(20^{\circ}\right)$

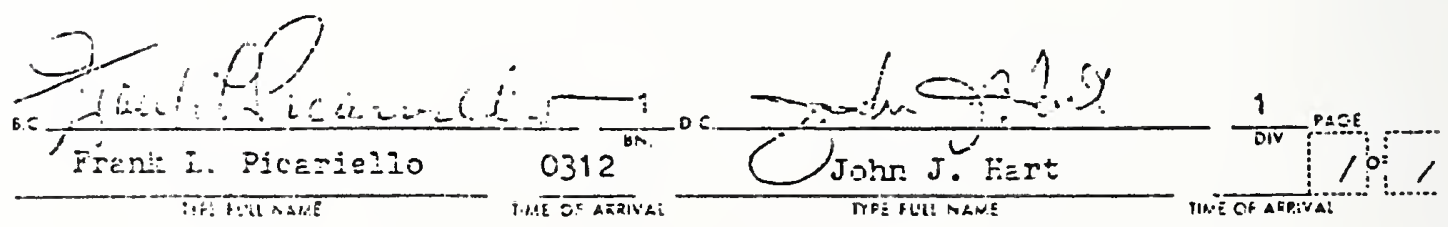



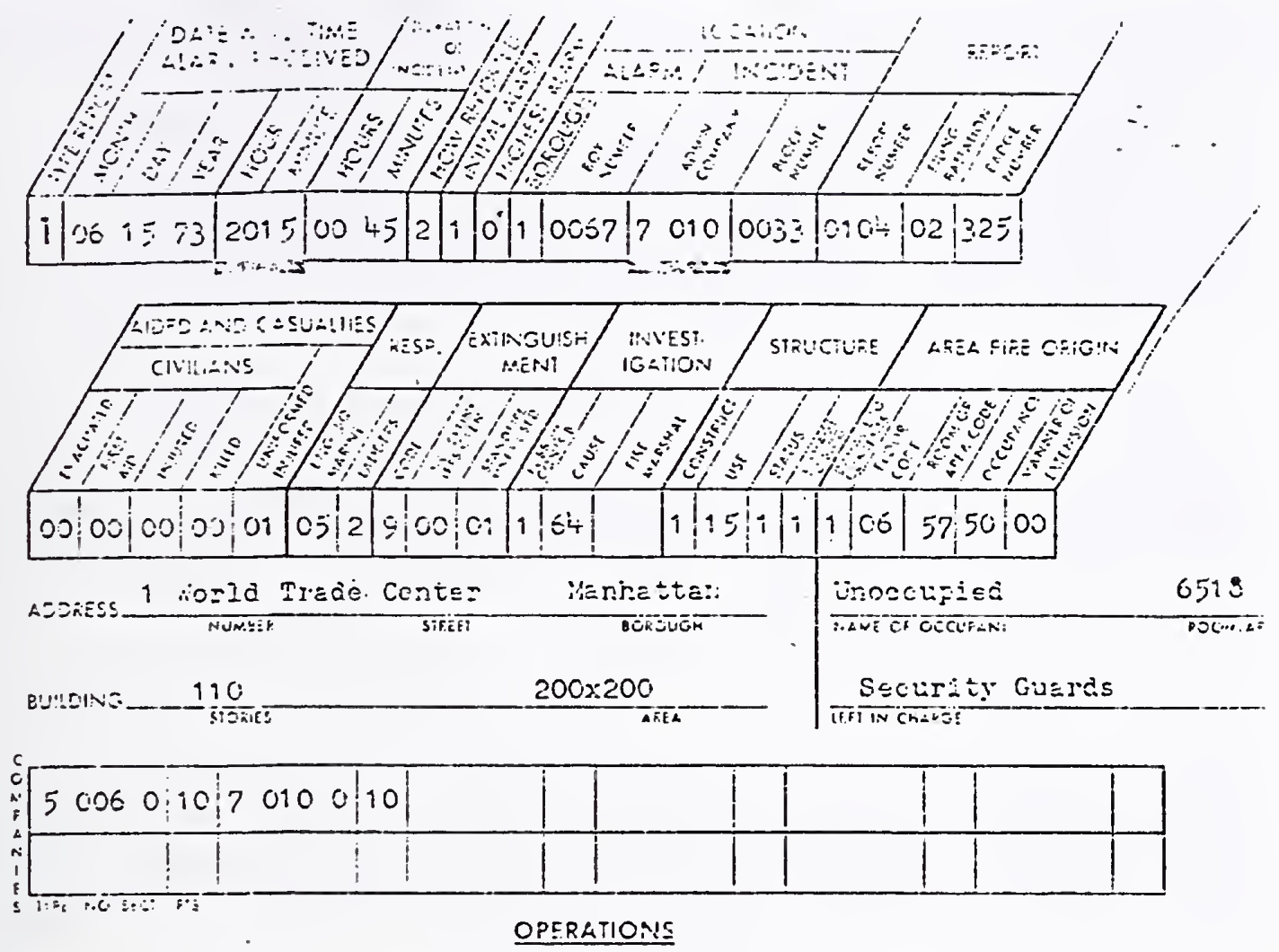

ipon arrival solnc rige ir: rub: ish in rook 65is On the 65th. fiosi,

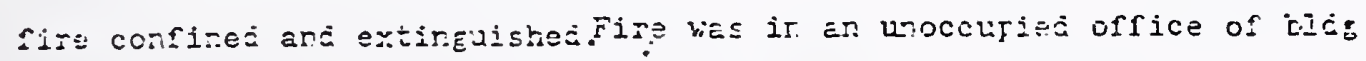

E.t: Siretohed lins off stancipe extinguished. fire,bad taren rollec ucs to 65th. floor.

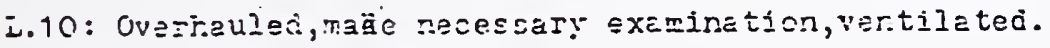

M Scere: Fire Fätrol ti2.

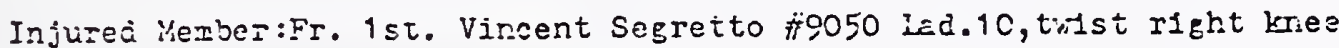
Dr. Schwerts notified.rio tixe lost.

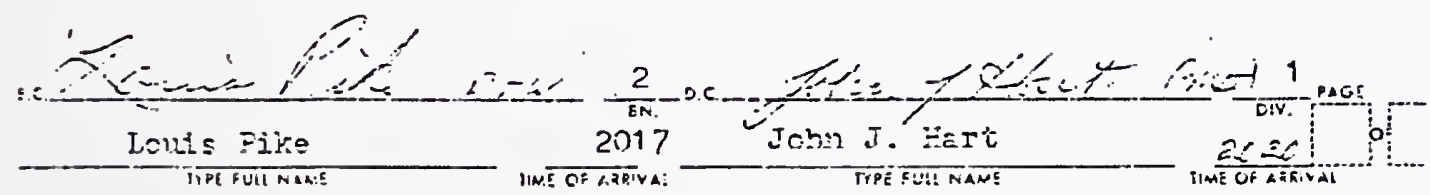

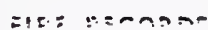




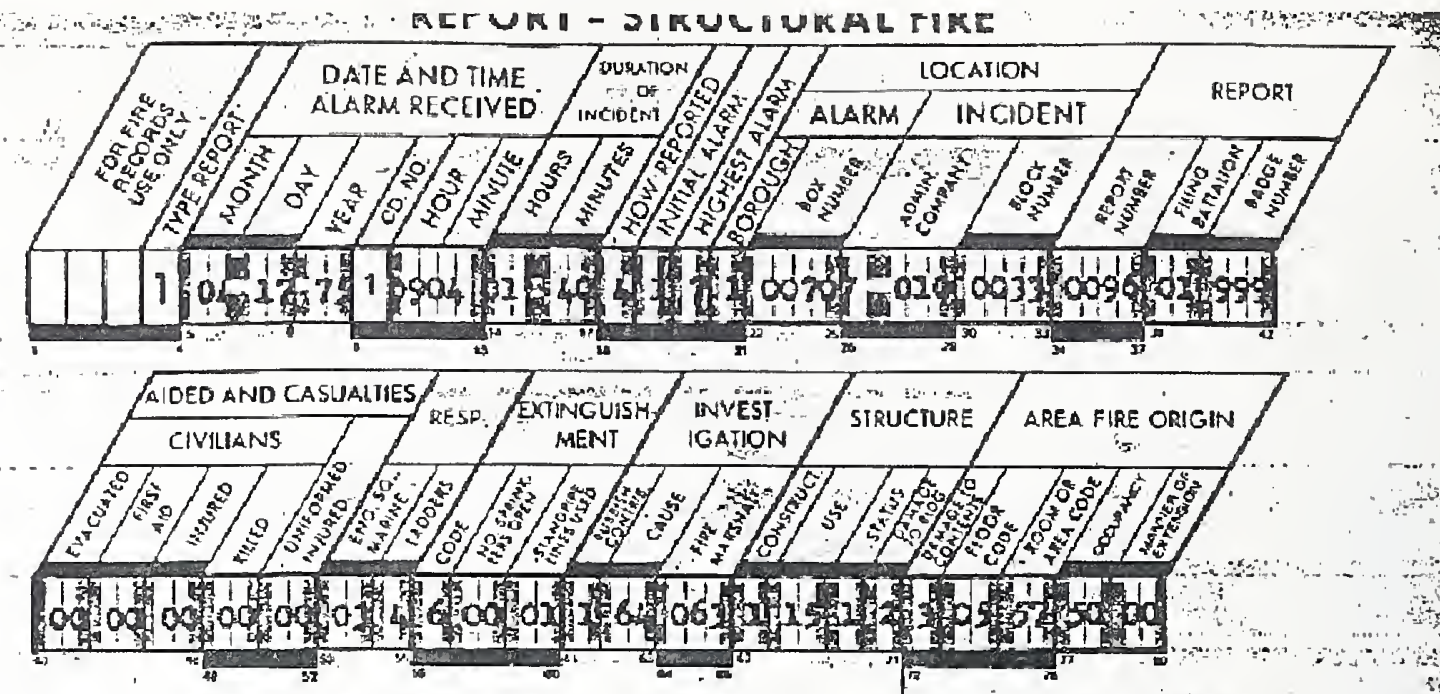

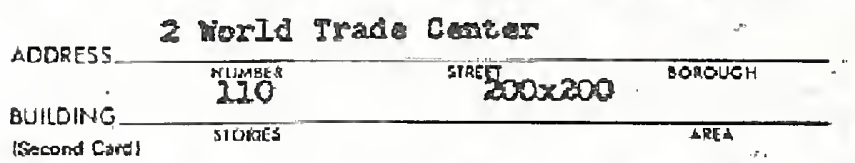

Part dubority

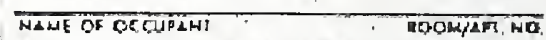

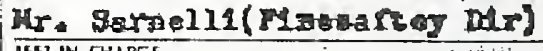
IIHT IN CHARGE , trecond Card

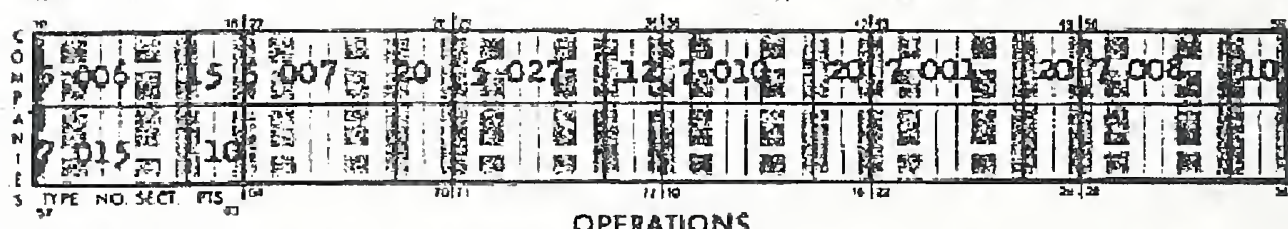

Becortrod Alaxa Clara $3-70-4$. OPERATIONS

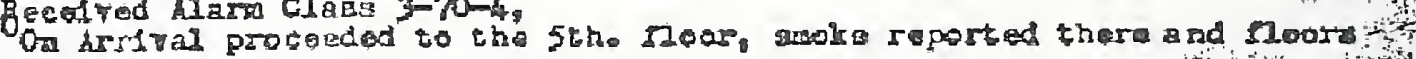
abover.

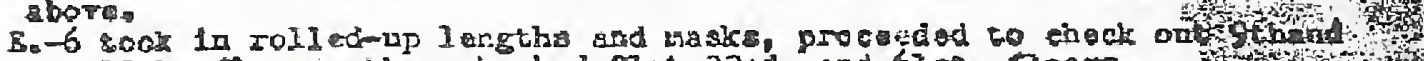

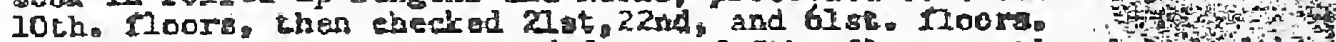

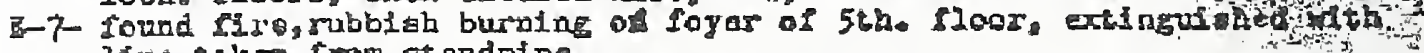
Ifre takea Irom btandpipa.

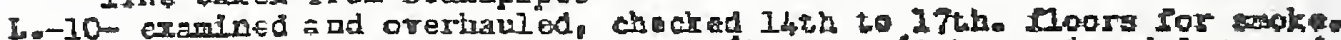

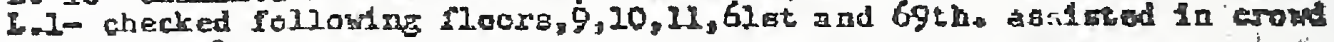
contrel.

E-27- checked 10th and 11ch. Cloos and assisted ln crokd controt. 1-8- chacked tho Both thra 998. Ilsor.

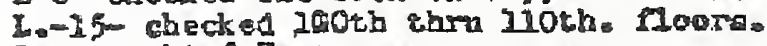

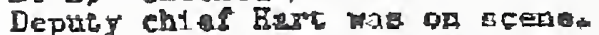

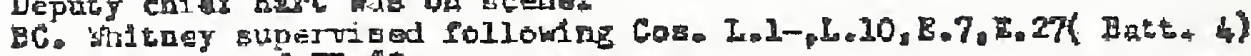

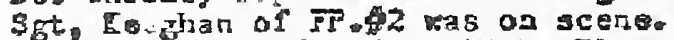

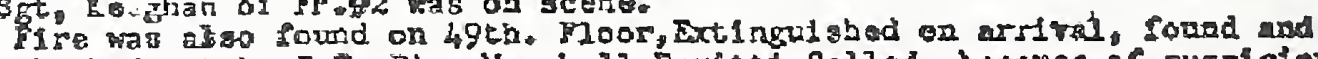

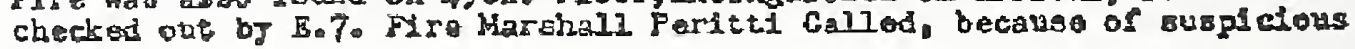
riso.

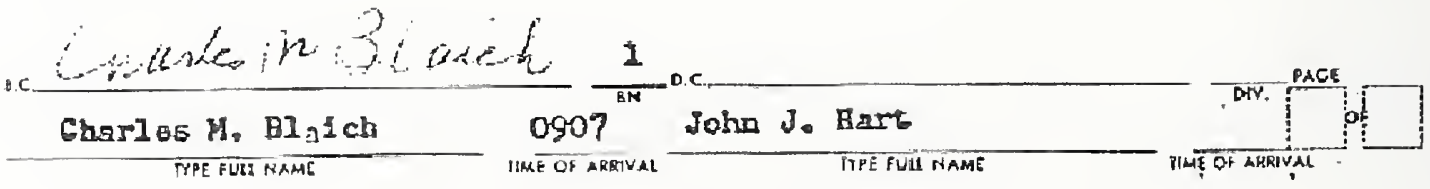




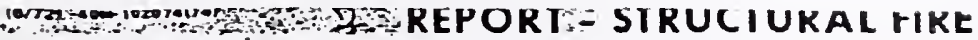
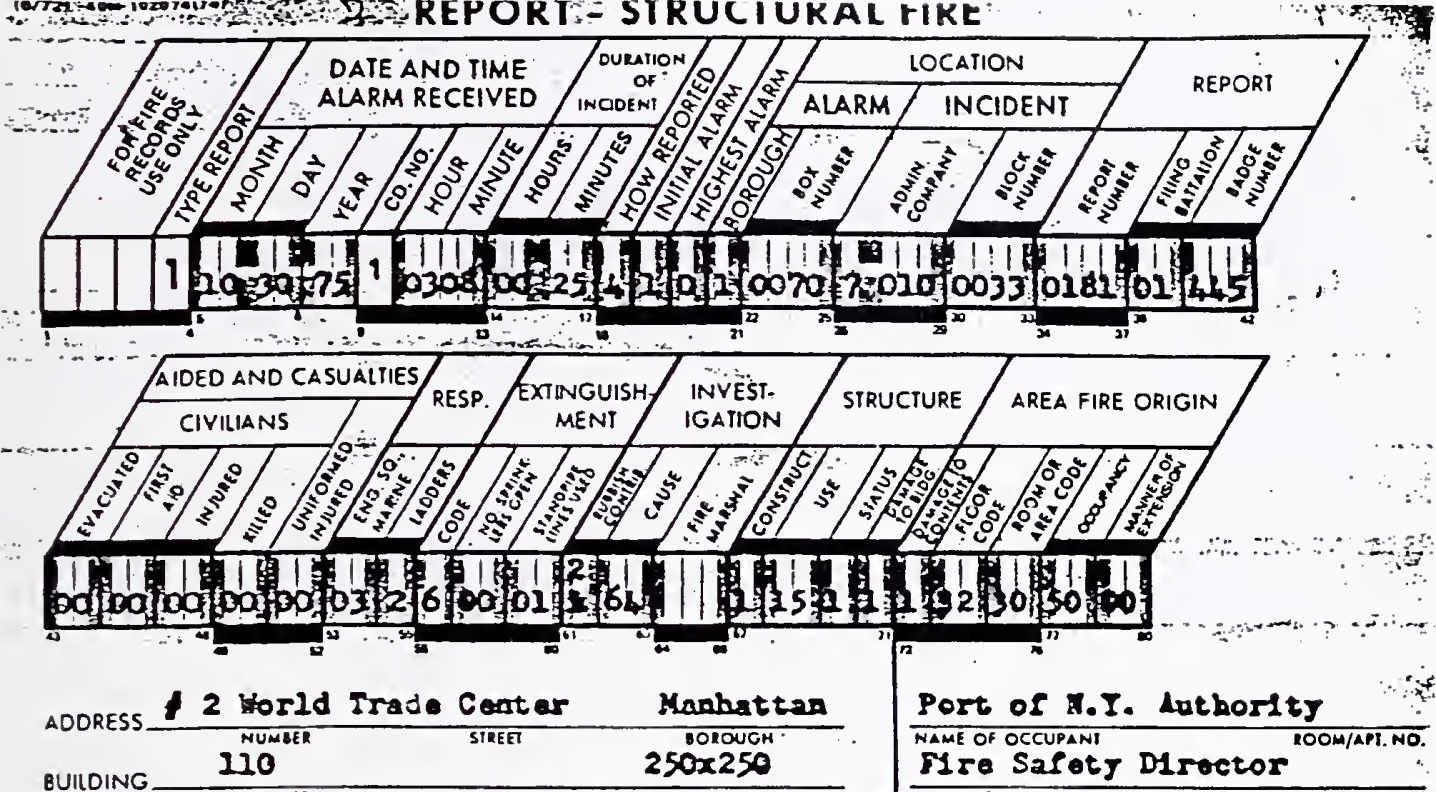

(Second Card) STORIES AREA

Fire Safety DIroctor

2018

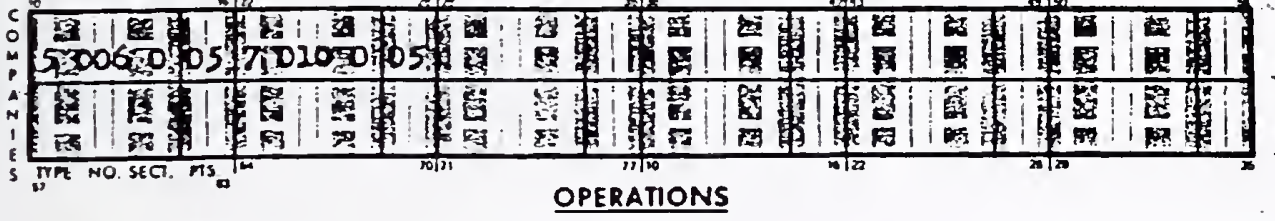

Besponded to Clas8 3-70-4

Opon arrival found fire in planter on 32 Noor there conflaed and Iitsighill extingal shed as follows.

E.6 - Carry in rolled up le:gbhs, hook up to S/P outlet, etrecte I1ne and operate on and extlnzulah f1re and necessary wash down.

L.10 - Make necessary exonnation of 32 and 33 noors and ovarbaul s. burat debris on 32 floor.

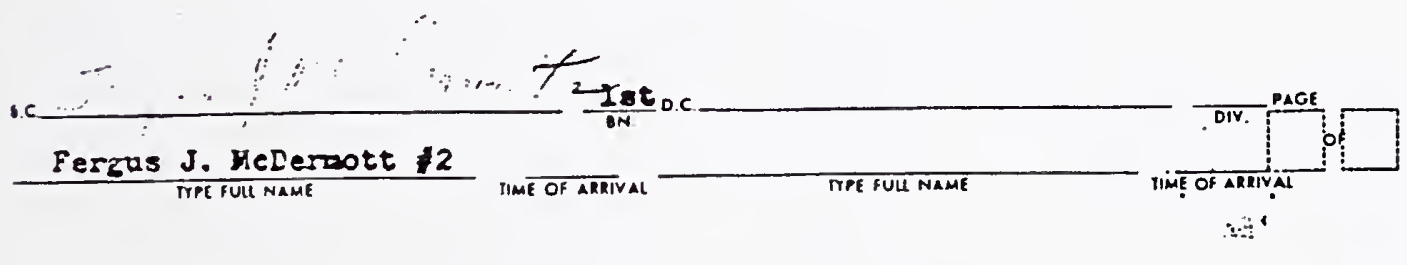



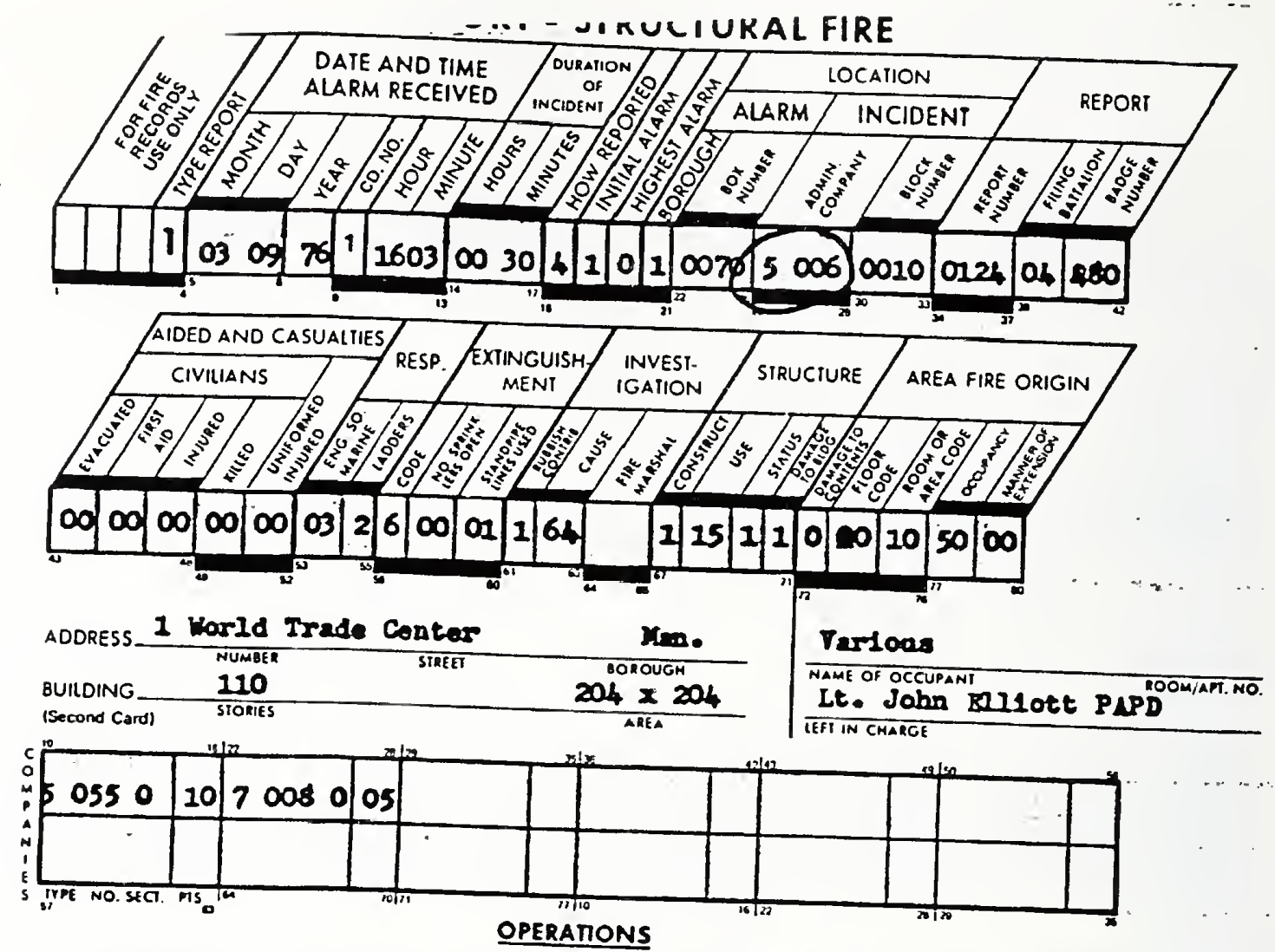

On 2rriral found 11ght firo condition in rabblah p1led agalnst wall in main concours wilch had caused scortching to sald all.

E 55 Stretchod Ine from standpipe outlet and ext1ngaished fire.

I 8 Examined for extension and winhauled.

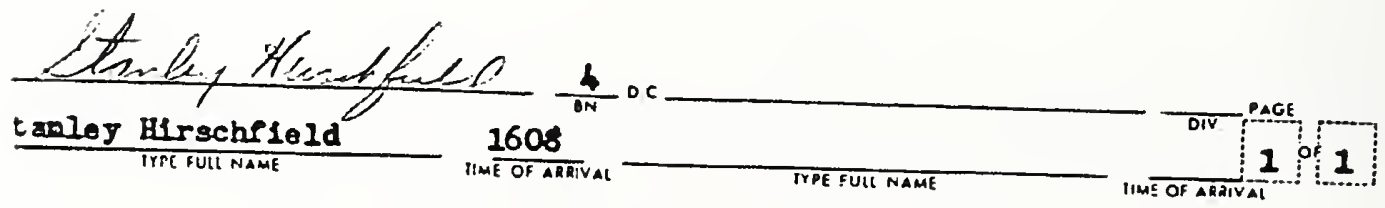




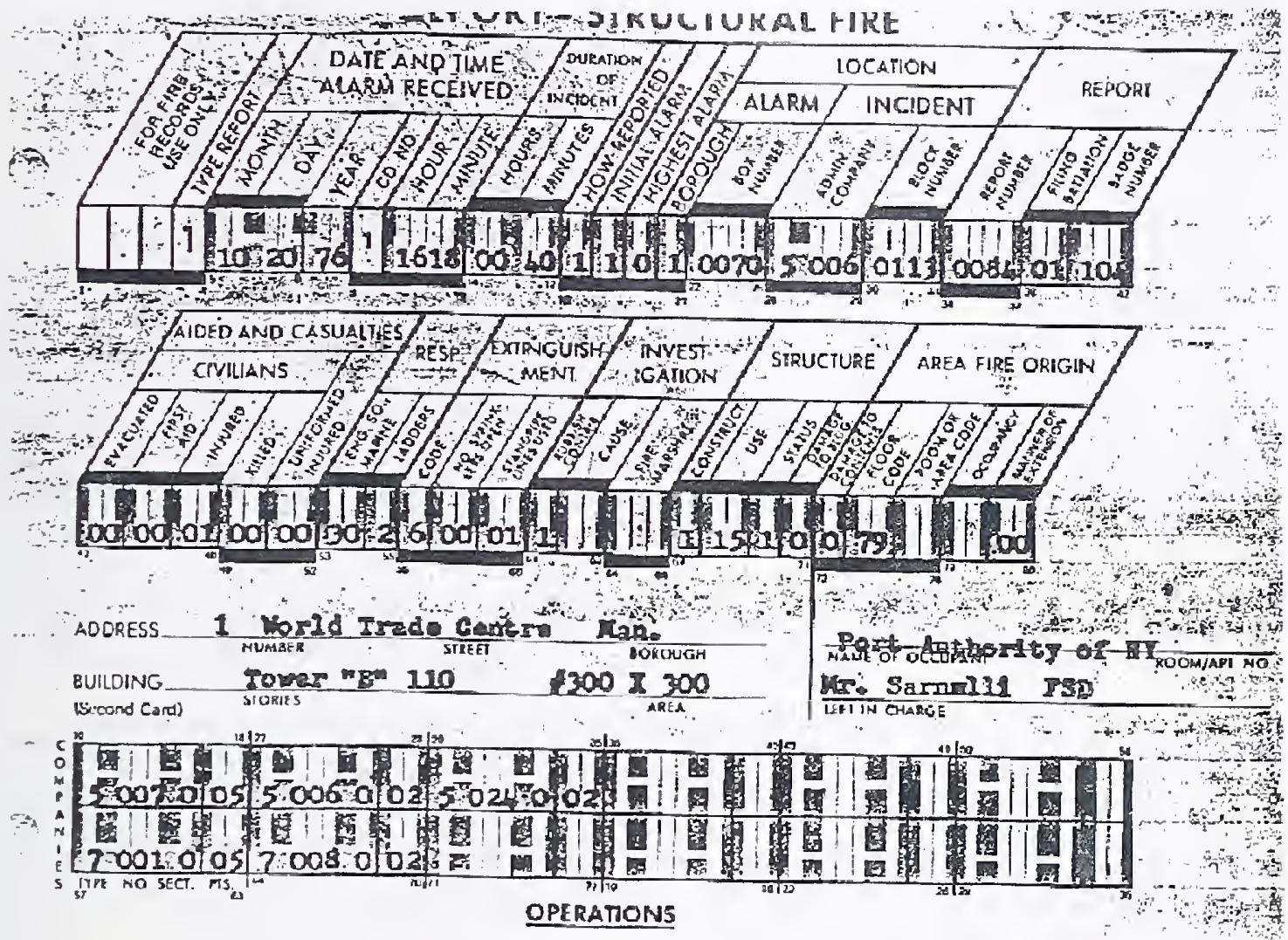

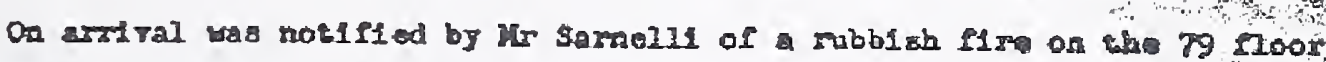
wouth wat cqudrent tower $B$.

5. 7 Responded to 79 moor etrecthed line from otandpipa Iro

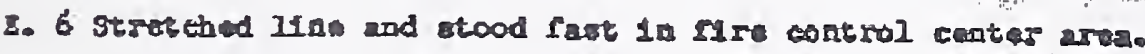

E. 2h Stretched Iino and stood fast In stalmy in lobby' area.

L. 1 Perforped vecessary search, ventlation and orer bau on Mre floors.

L. S stood rast wth toola and wastes in eteinay at 10sbyo

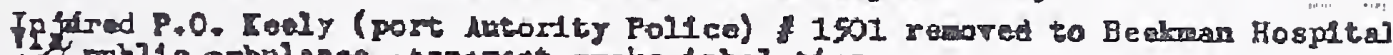

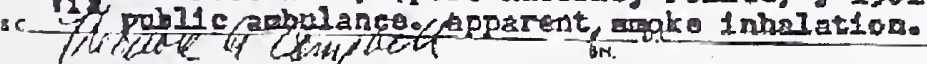

Thoodorat Campbel $\frac{1620}{1620}$

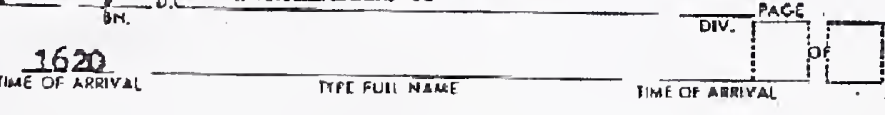



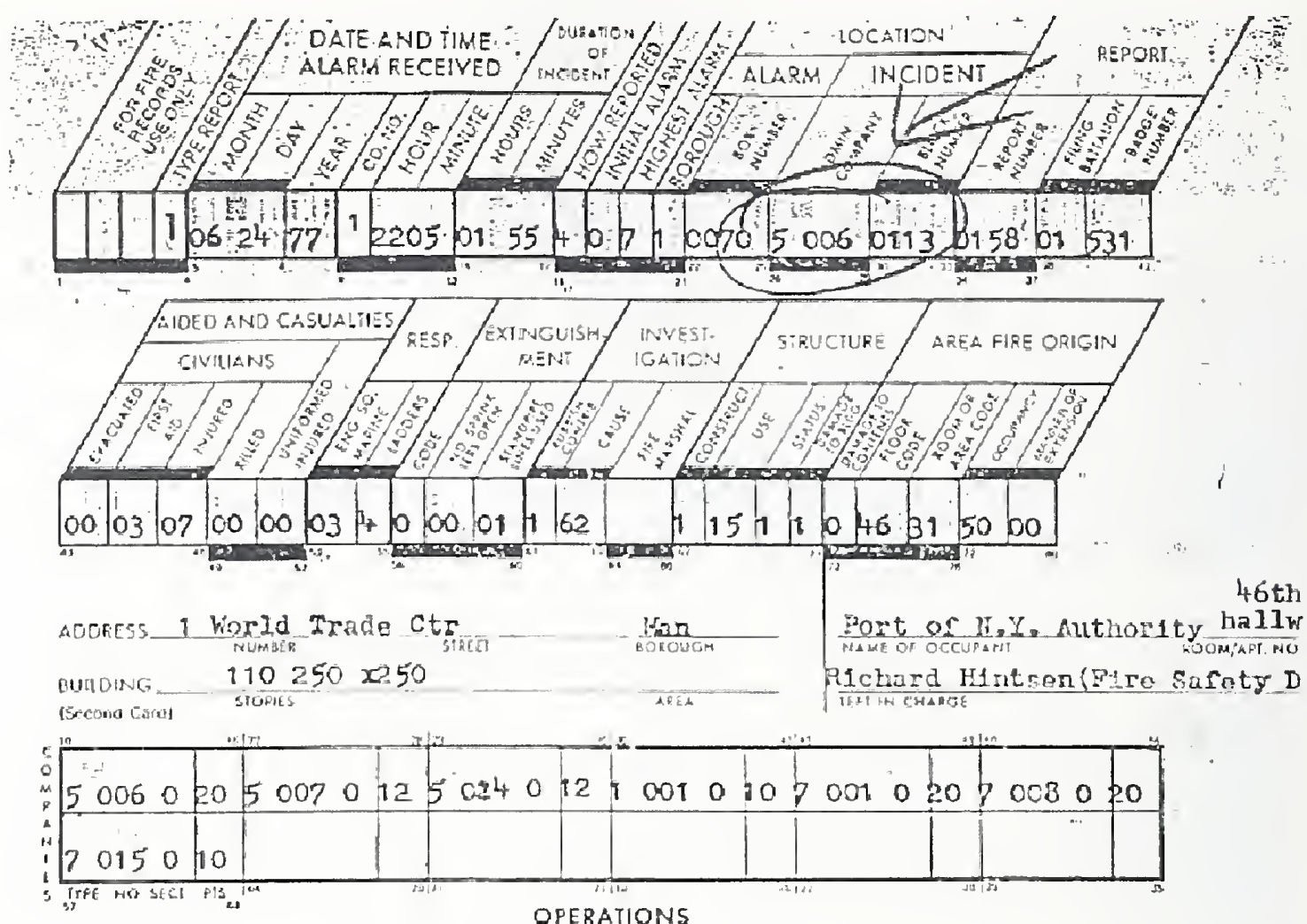

Responded to $3-70-2$ (ranual Alarta)

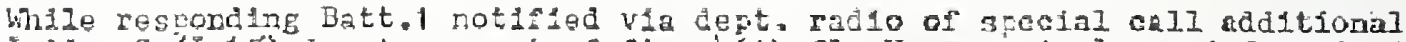

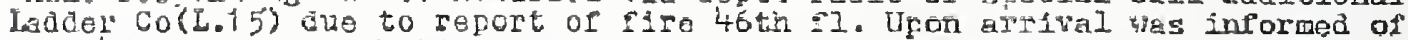
fire 46 th fl public haliway near r=e1ght elevator. Ordered investigation a nd found fire therein, which had been extingusbed prior to the arrival of this dept. Eqacuation instituted by port suthority personiel prior to arrive 1 of Fire Dejt. unitg. Report of saoke detector oparational of the 103 ra 91 . Fire located betreen ireight elavetors 49 \& 17.

E.E-Rolied up lengths to the 4hth II. Correated to stardpipo thereln and stretched to Ilse floor(46th) hasked cown fire area fot orerbuling purposes.

E.7 = Aspisted In stretch, thon ortered to searoh, exanination of 53 rd to

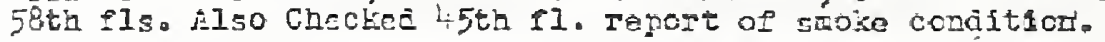

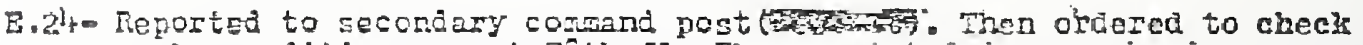
rerre of 535 do 56 th flg. Also chectied out seoke detector 103 Id Pl.

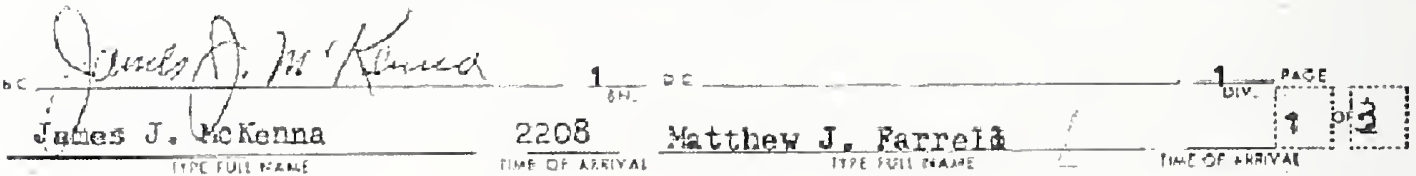




\section{REPORT - Additional Data}

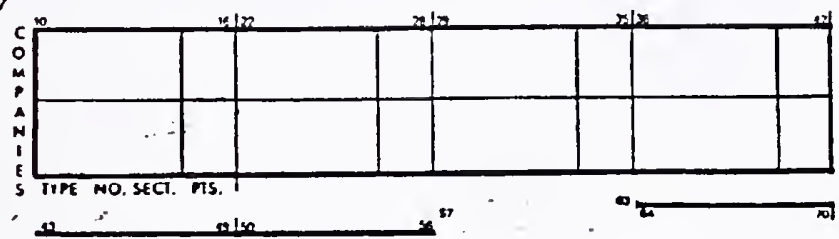

\section{OPERATIONS}

I.t- Inftially to the flre eloor (46th) operatod for orerhanling search

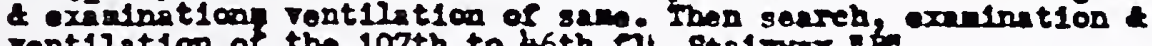

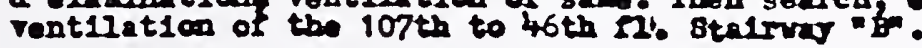

I.8 Inftility to secondary connand post. (West st). Thon ondered to chock 78 th to 60 th 918 search \& examenation. 180 assistod in overhaulins P1re. Iloor (46th)

I.15- Spec1al called to report to West 8t. Imen trough lobbs to secondary command post. Benrout found (2) elvilian cloaning personnol (female). hed been renored to lobby saffering smoke inhalation. Performed first Ald an infured c1rlilens. Then rellevod by Ros. I with resostitatar Then rack ordered to serache 47 th to $52 \mathrm{nd}$ fis as (2) infured e1villans reported to bin re vorked an the $48 \mathrm{th}$ s0th $12 \mathrm{~s}$.

Bes.1- Rellored I.15 and adminlstered first ald(Inhelation)to Injured civilens. Then ordered orfice $I$ \& remainder of menbers rent to

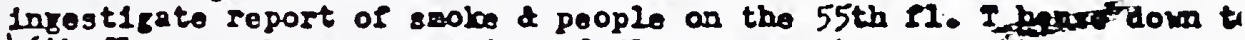
46 th 11 . Seerch, exa dnation of rloors enroute.

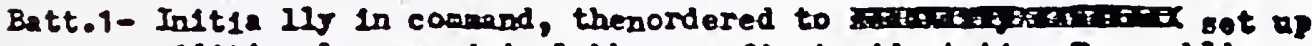
additional comand in lobby, as first ald station.two public anblances standine by vith (4) resusc1tators. Directed search operations of B.7, L.15, Bes. 1. Ordezed swote parge 45th to 107 whon fire vas out.

Bett.2- Ordered to supervise undts on the flre floor a report conditsons there1n. Supervised in pert operation of $B .6, I_{i} I$

Batt.32 - Ordered to supervise operations of antts above fire floor.

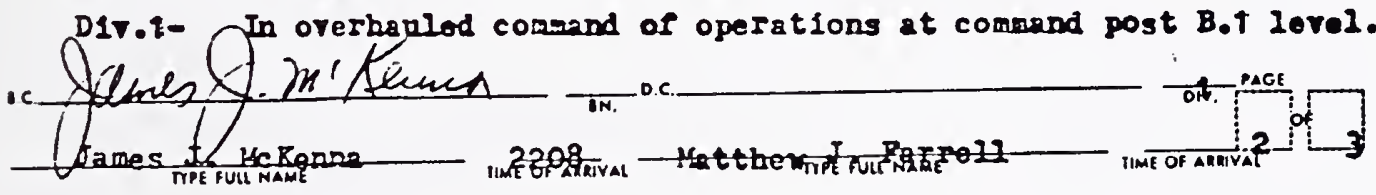




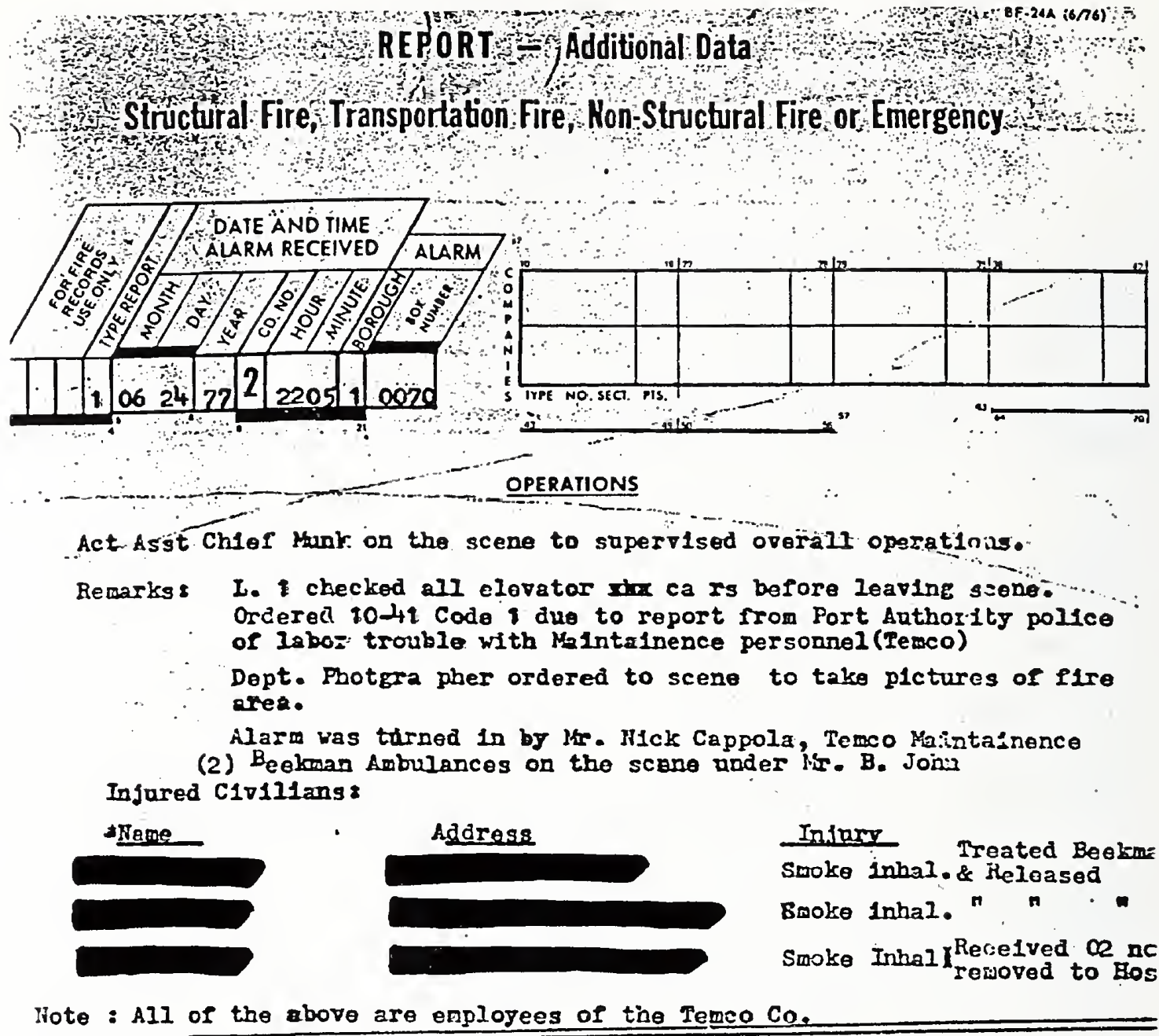

Sgt. Steve Fox Bdf 264 Burs to Fingogrs Rt Hrind.

Ptl A. Hallcker

Barn to Rt hist

Ptl Mejers

Snoke inhal

Pt1. Careale

Snoke Inglel.

Noter Pollce members of Port Authority Pollce Dept. All Injured treated at Beobren Bospital \& releasod.

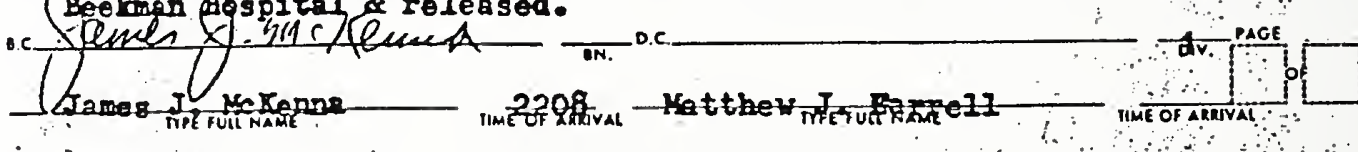

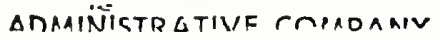




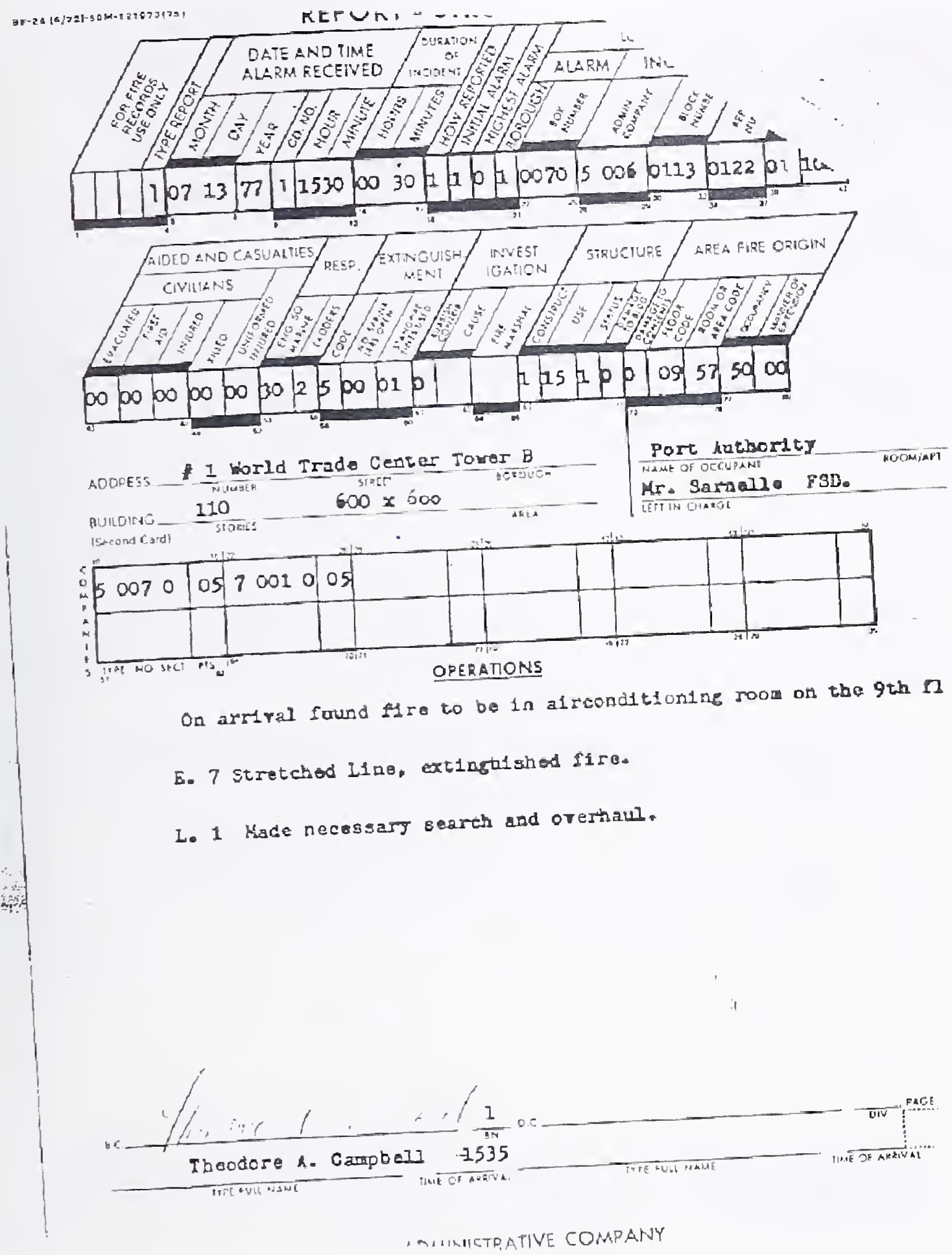




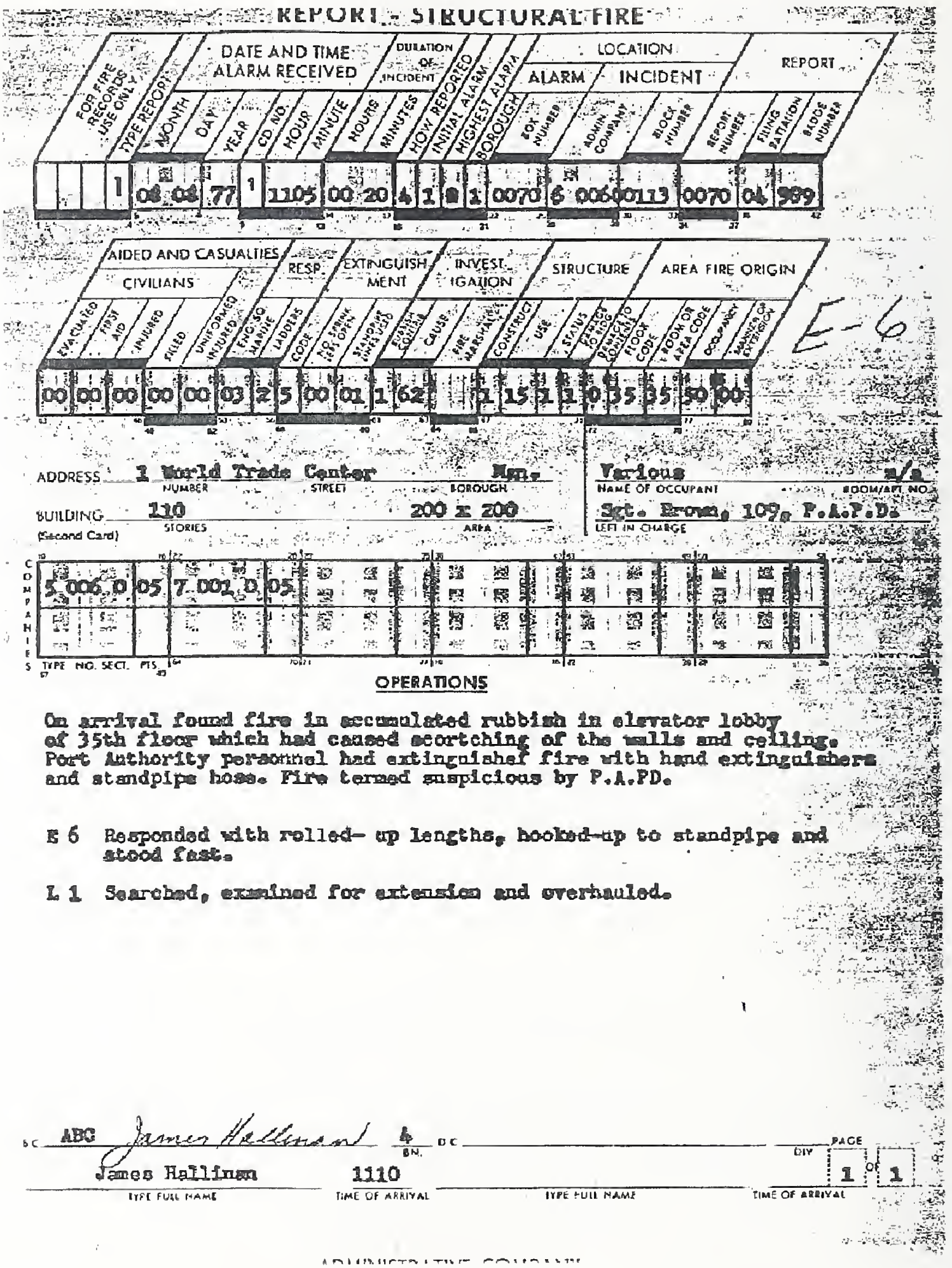




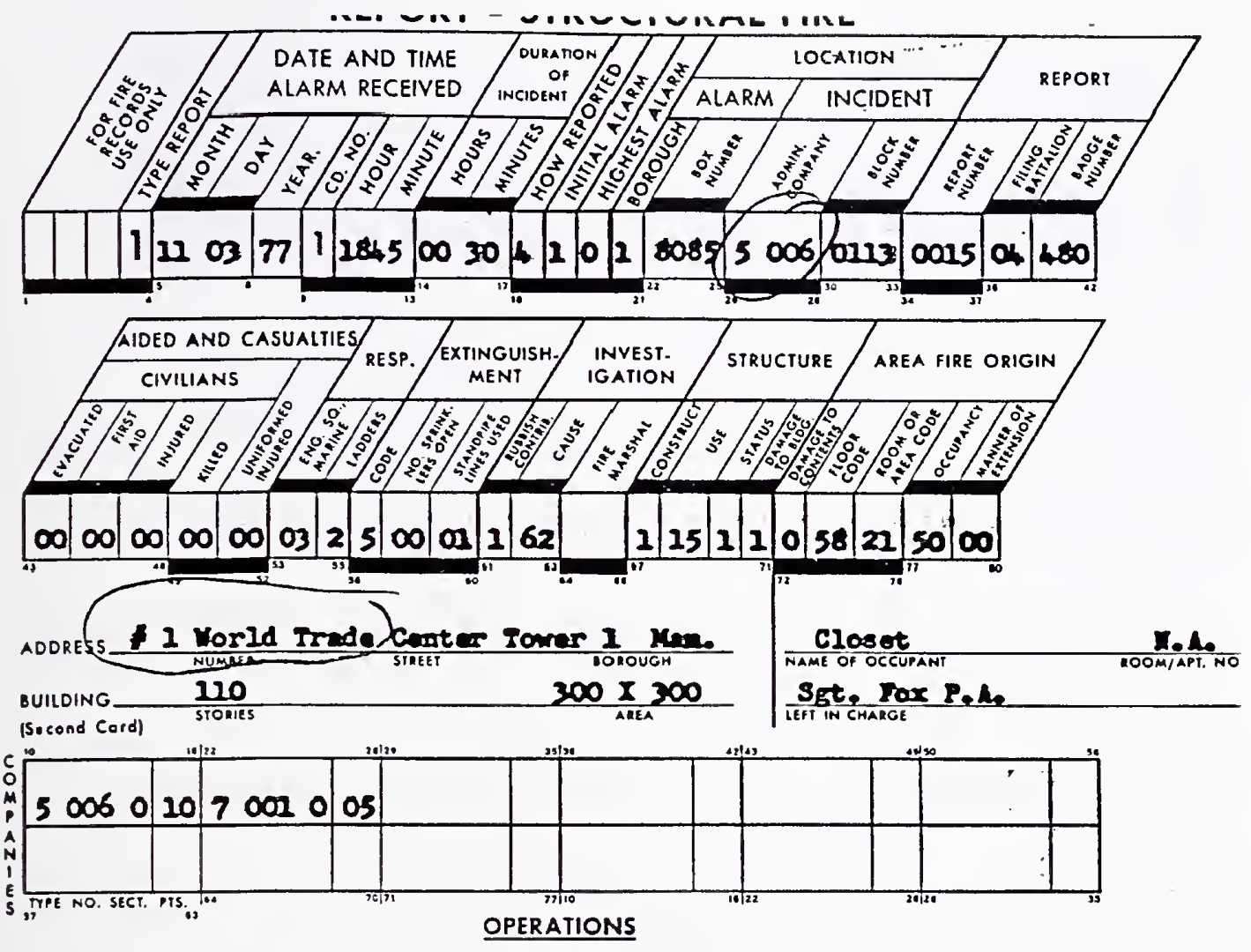

On arrital found cause of alara to be elro in jemrtorial closet in rolring rags and rubblah. Batt 4 requested a $10-41$ codel F1ro cansed seerlng of paint on wils and celilng.

E-6 Stretched rolled up langths and hooked up to atandplpe out Iet and ext Ingulshed firo.

In Mad exastation of wall and celling ad wexllated a nocesary.

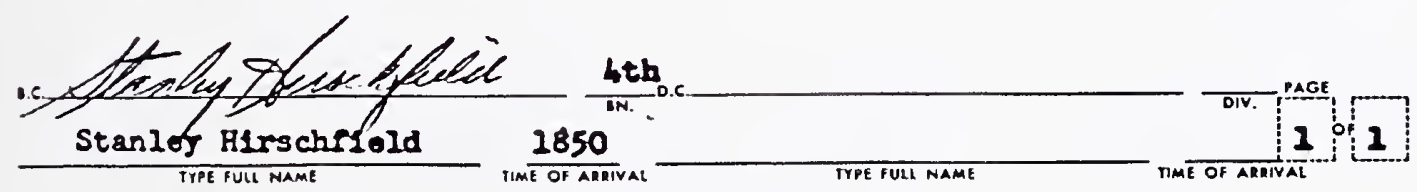

ADMINISTRATIVE COMPANY 

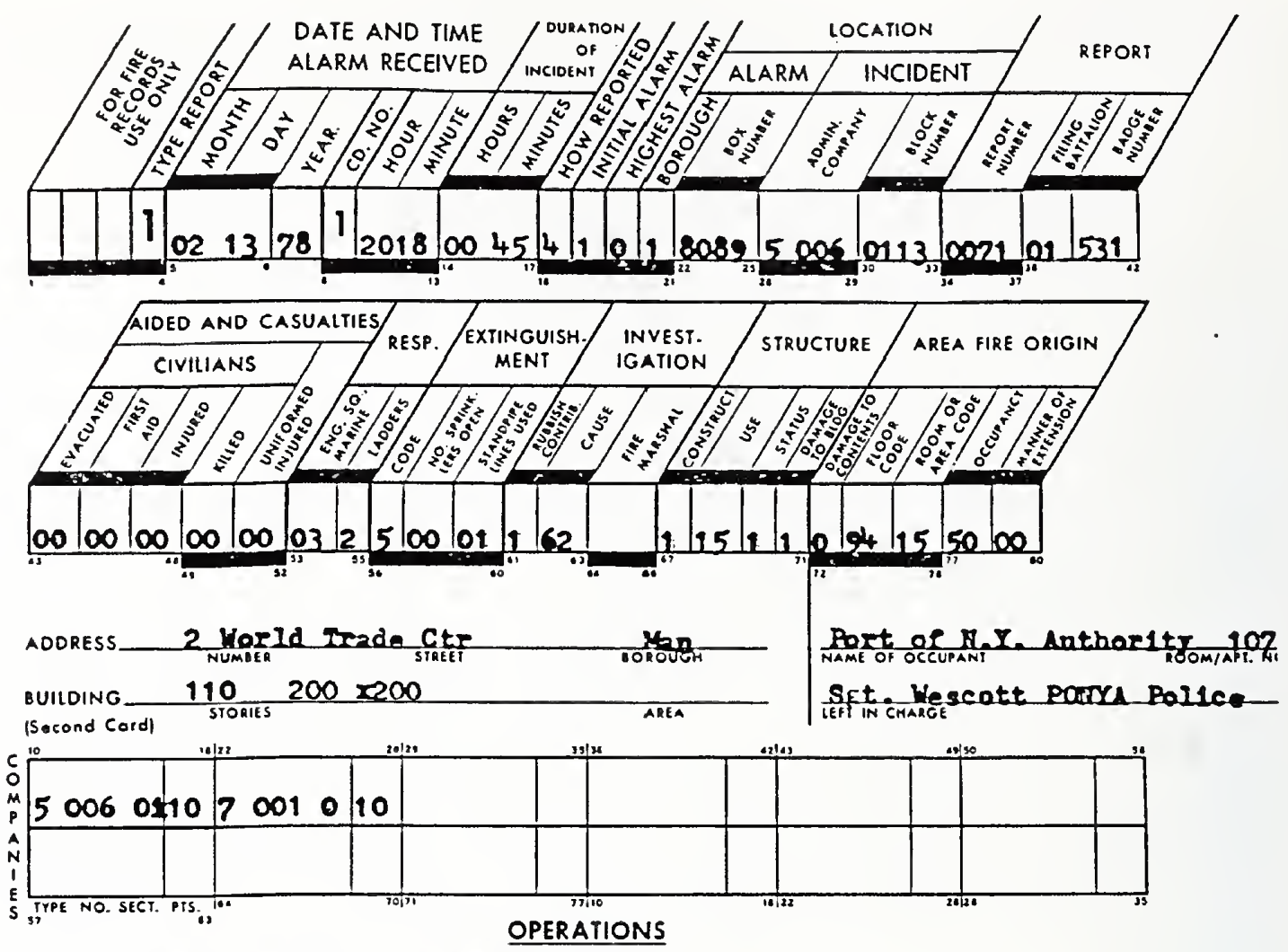

Responded to mavel ela ra box 8089-

Upon arrivel wes 1nformed of f1re 107th 1 . Ordered infestleation and found fire in rabe1sh \& aint. materials therein. Batt. 1 ordered adaitlonal Battalion Chlef to rospond on report of derinate fire. Batt. 4 responde d.

R.6- Rolled up lengths to the fire rloor extingulshed ramalntag firo

L.1- Search, examination of Ifre floor \& floor above. Opanod walls for oxemination. Overhauled burned materials.

B.4- Ordered to supervise operations on tho flre floor.

D1v. 1- Responded to scene, and assumed comand.

Note: Batt.1 transalted $10 \alpha_{1}$ Coie 2 \& requested FiM. to respond.

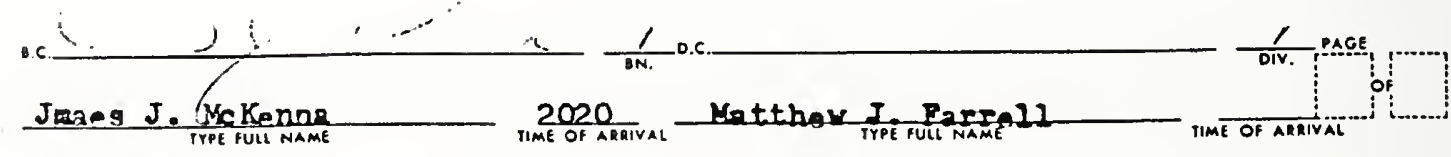

ADMINISTRATIVE COMPANY 

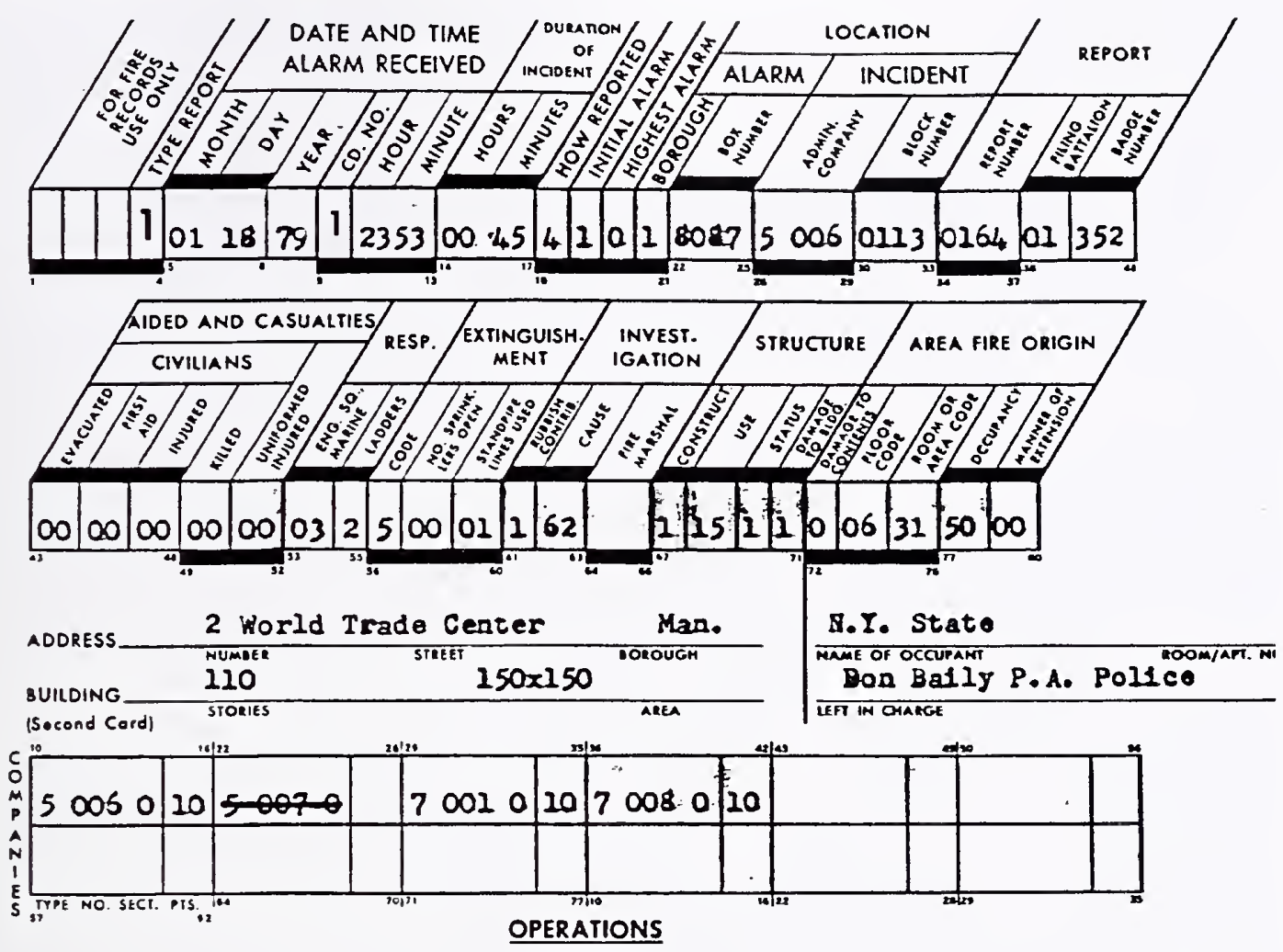

Upon arrival was told of flre on the 6th floor, operations as follows. Ladjer 1 made necessary investagation, located the fire, vented, overhauled and searched.

Ladier 8 searched and rented floor above, overhauled.

Engline 6 stretched a Ilne from standpipe and extinguished the fire. englne 6 washed down.

Batt. 2 on the scene.

D17. I on the scene.

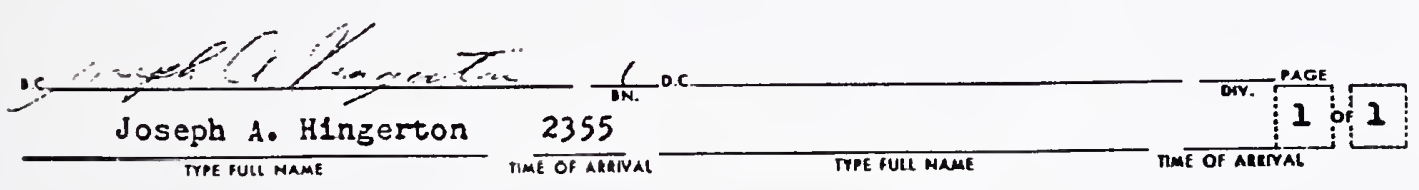




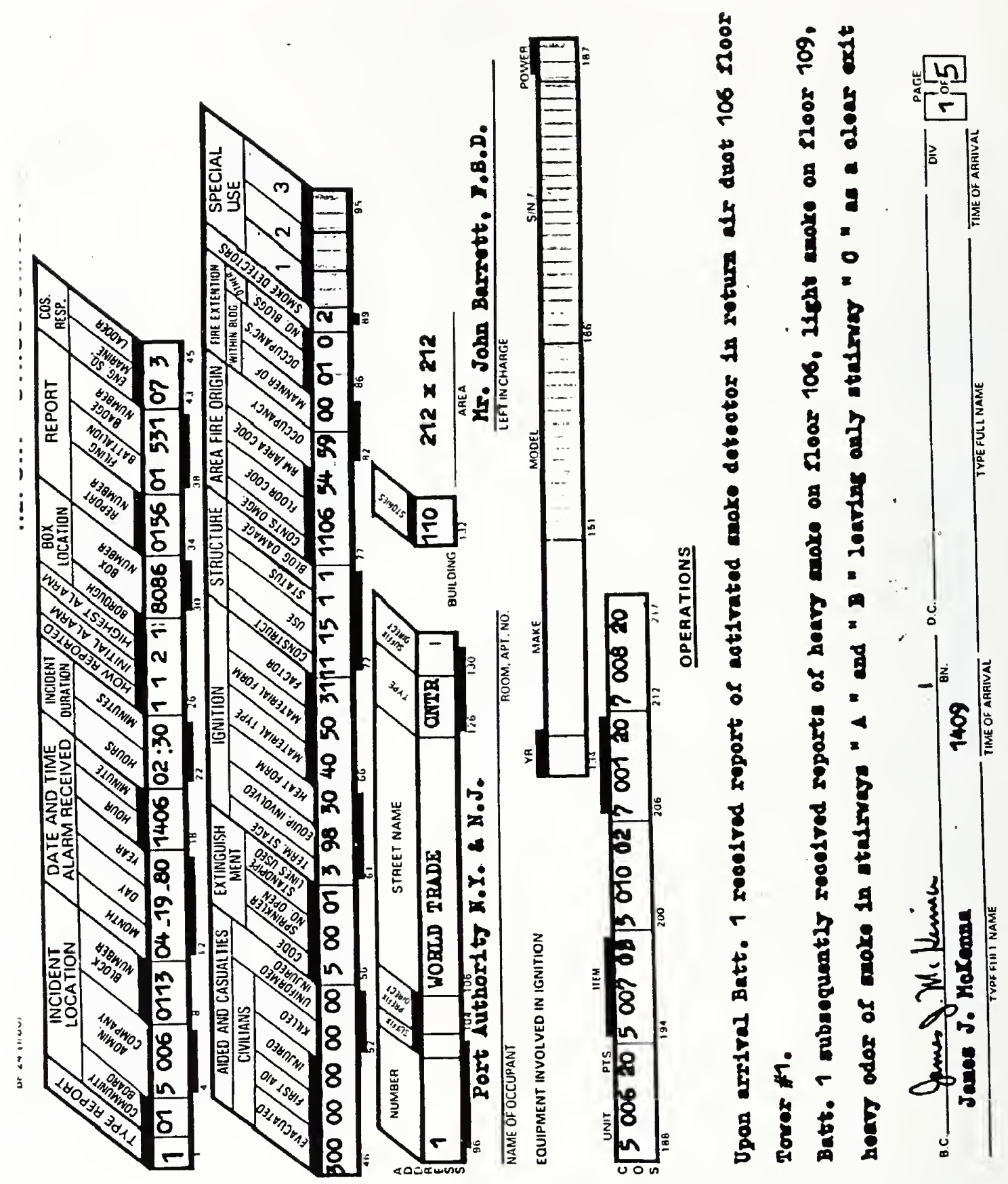

ADMINISTRATIVE COMPANY 


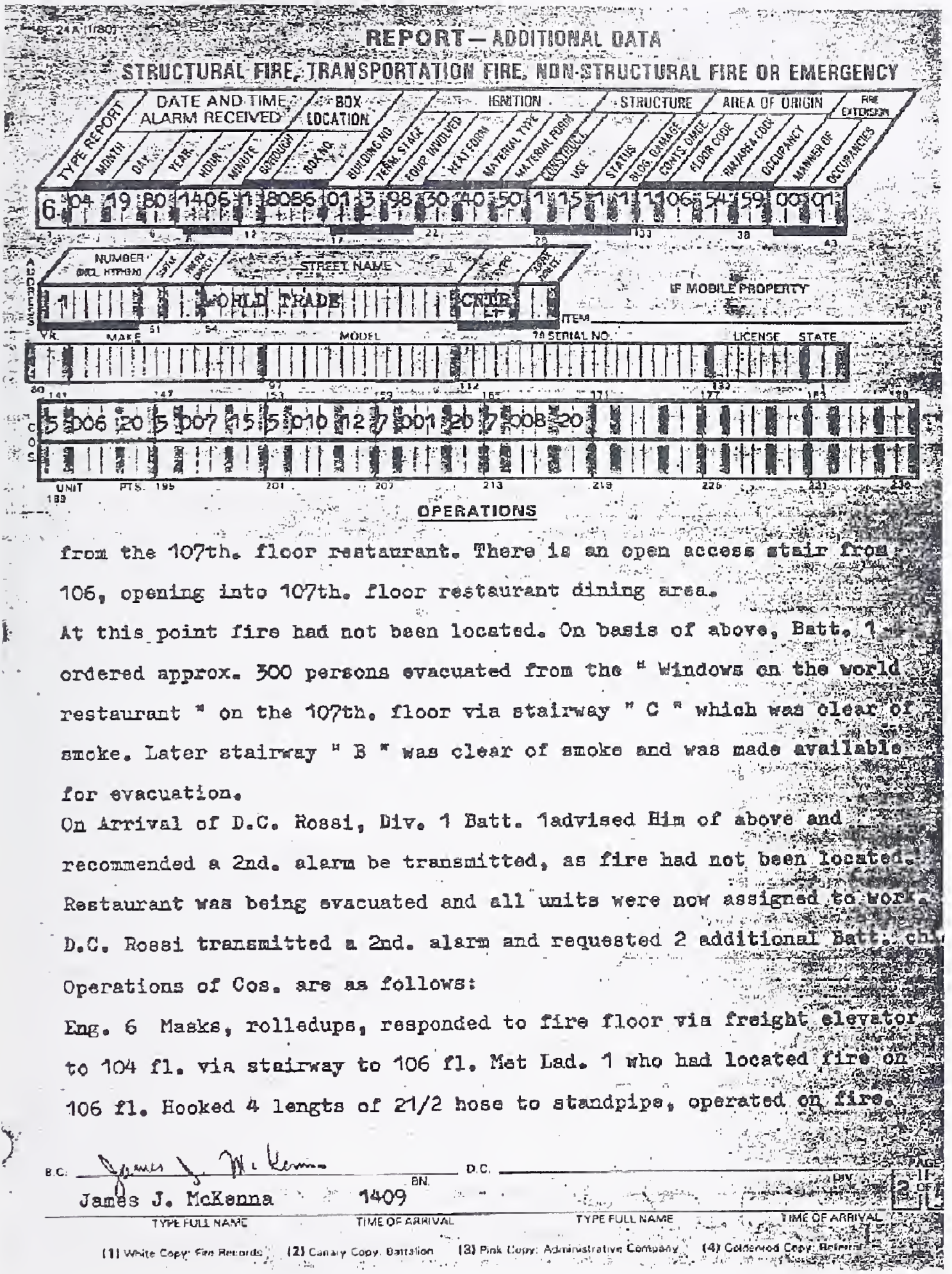




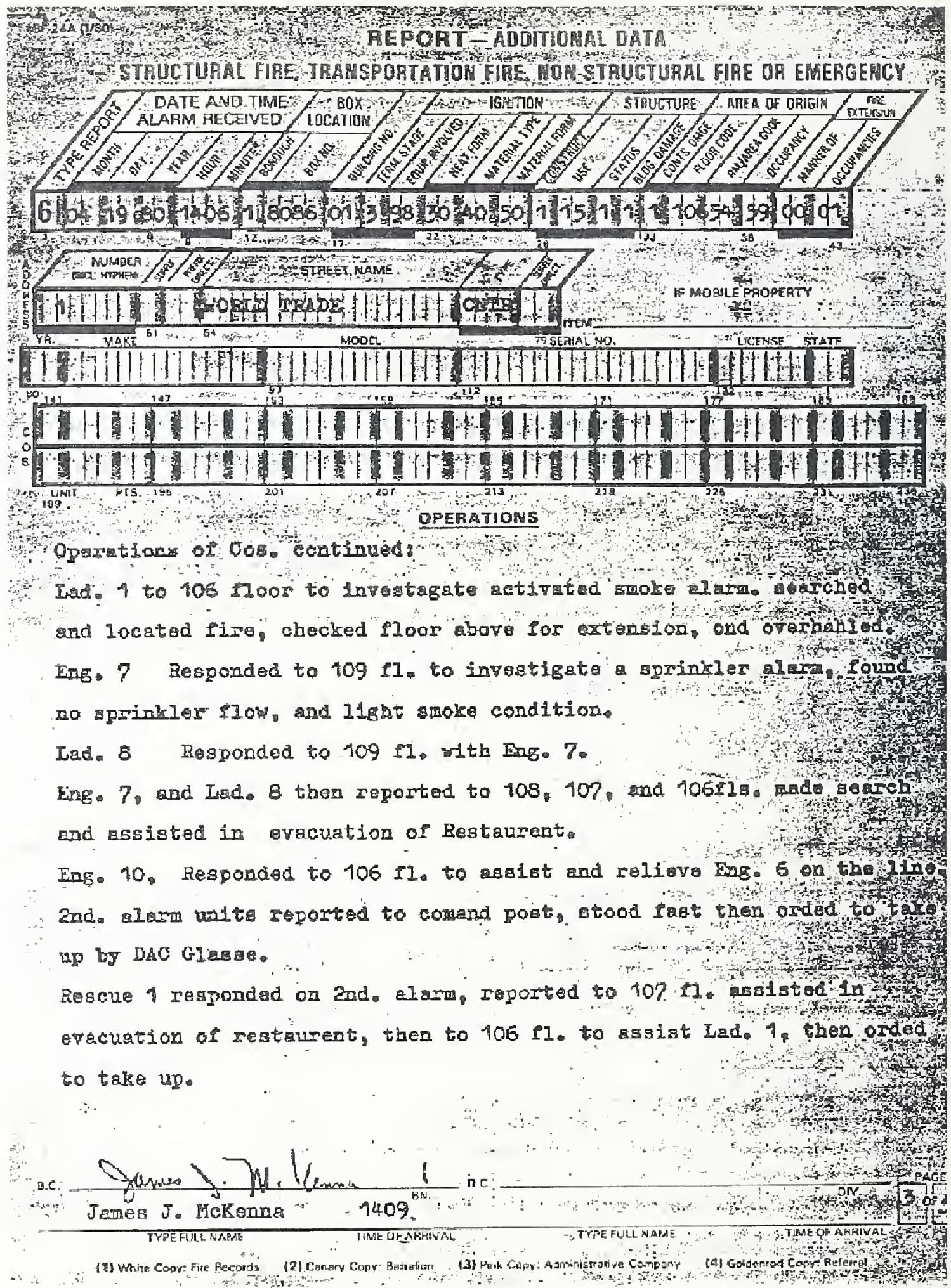




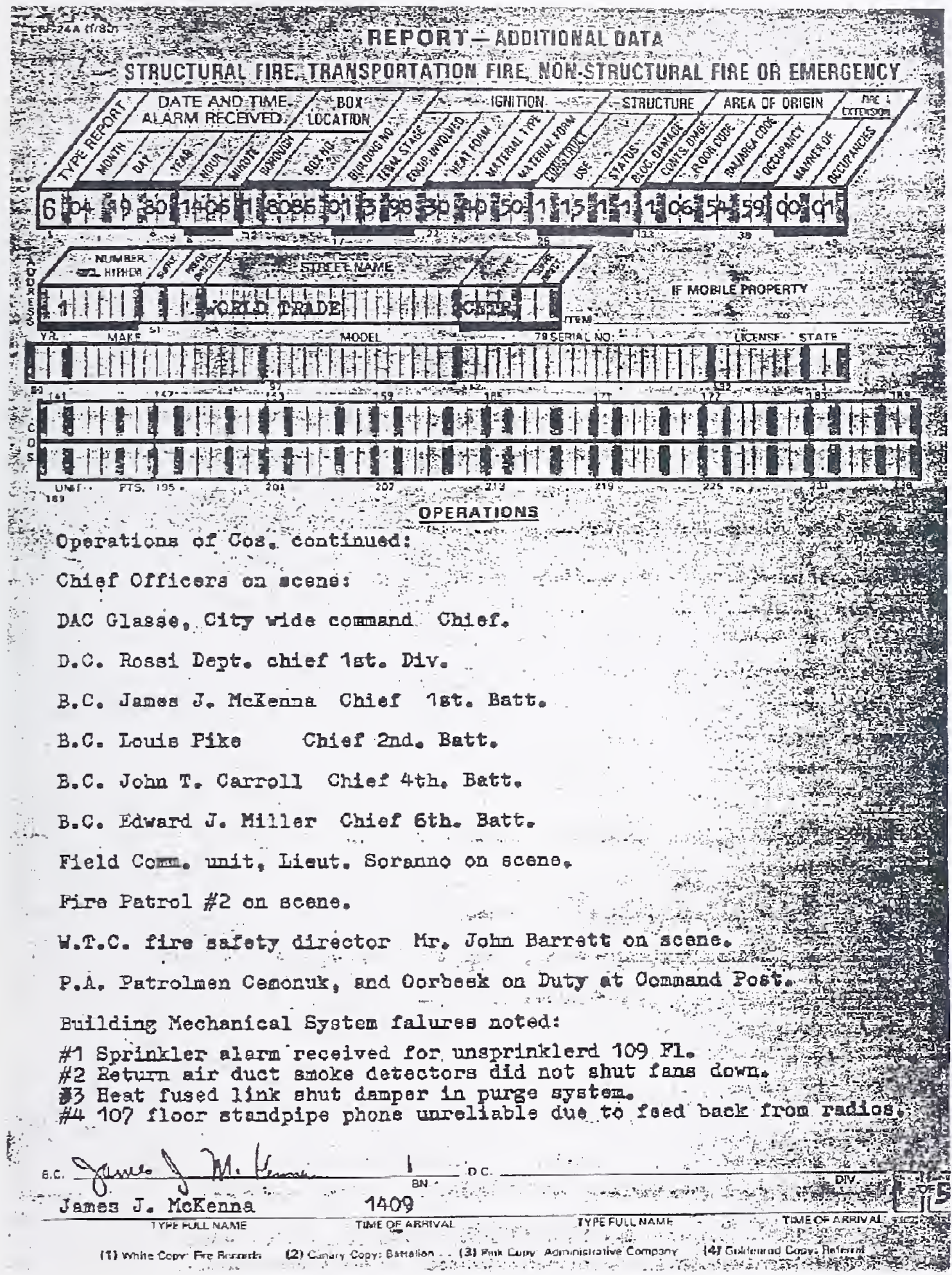




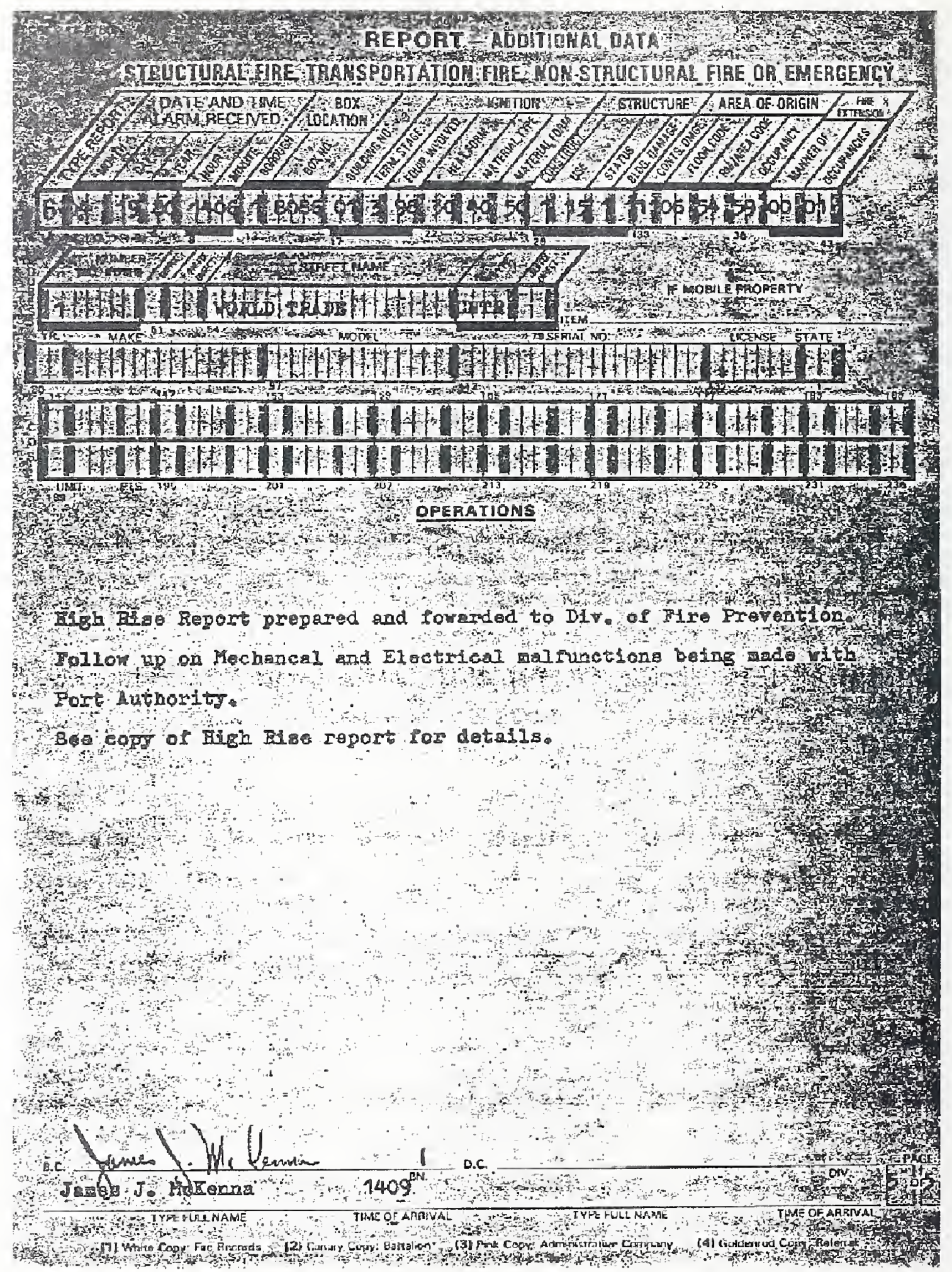




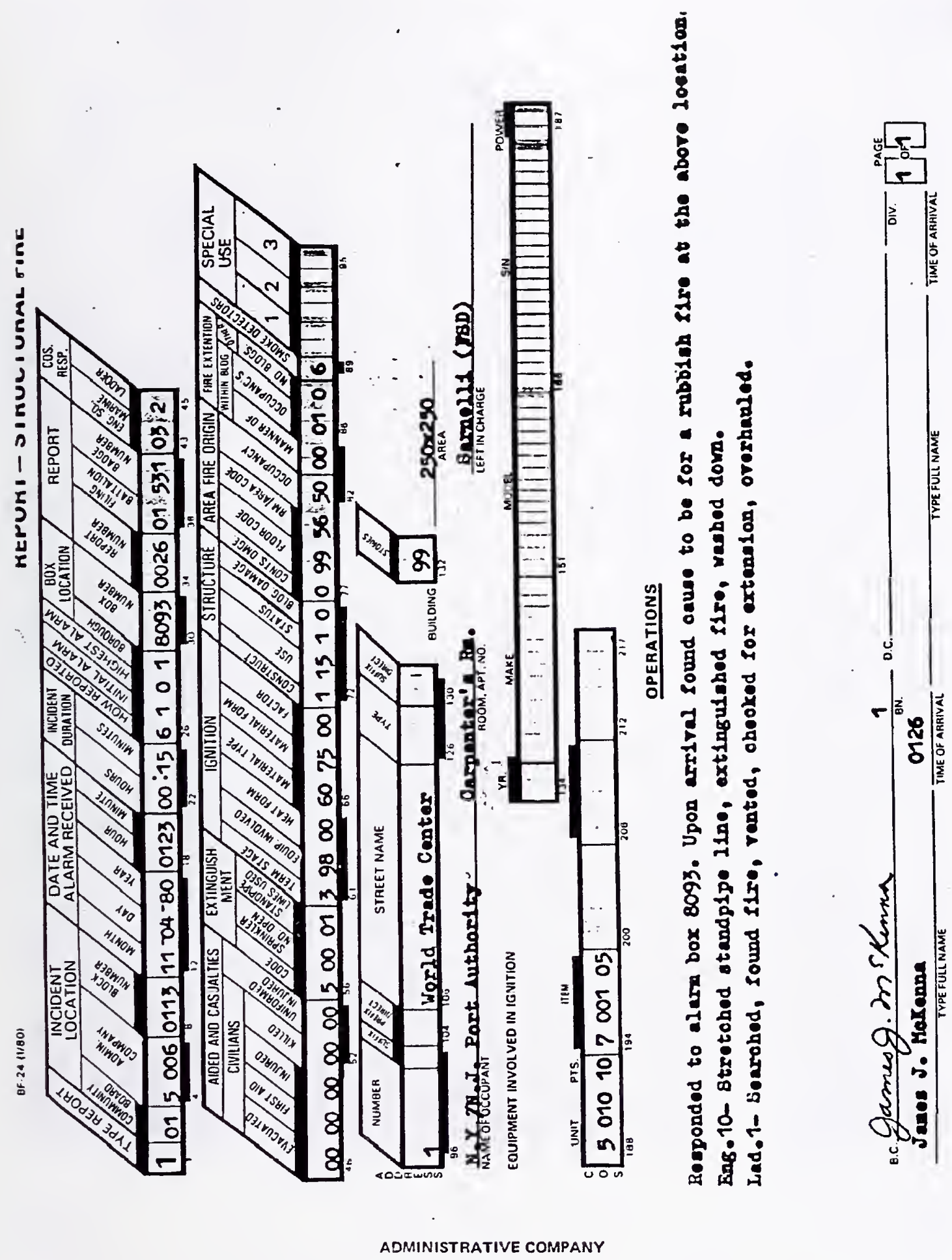




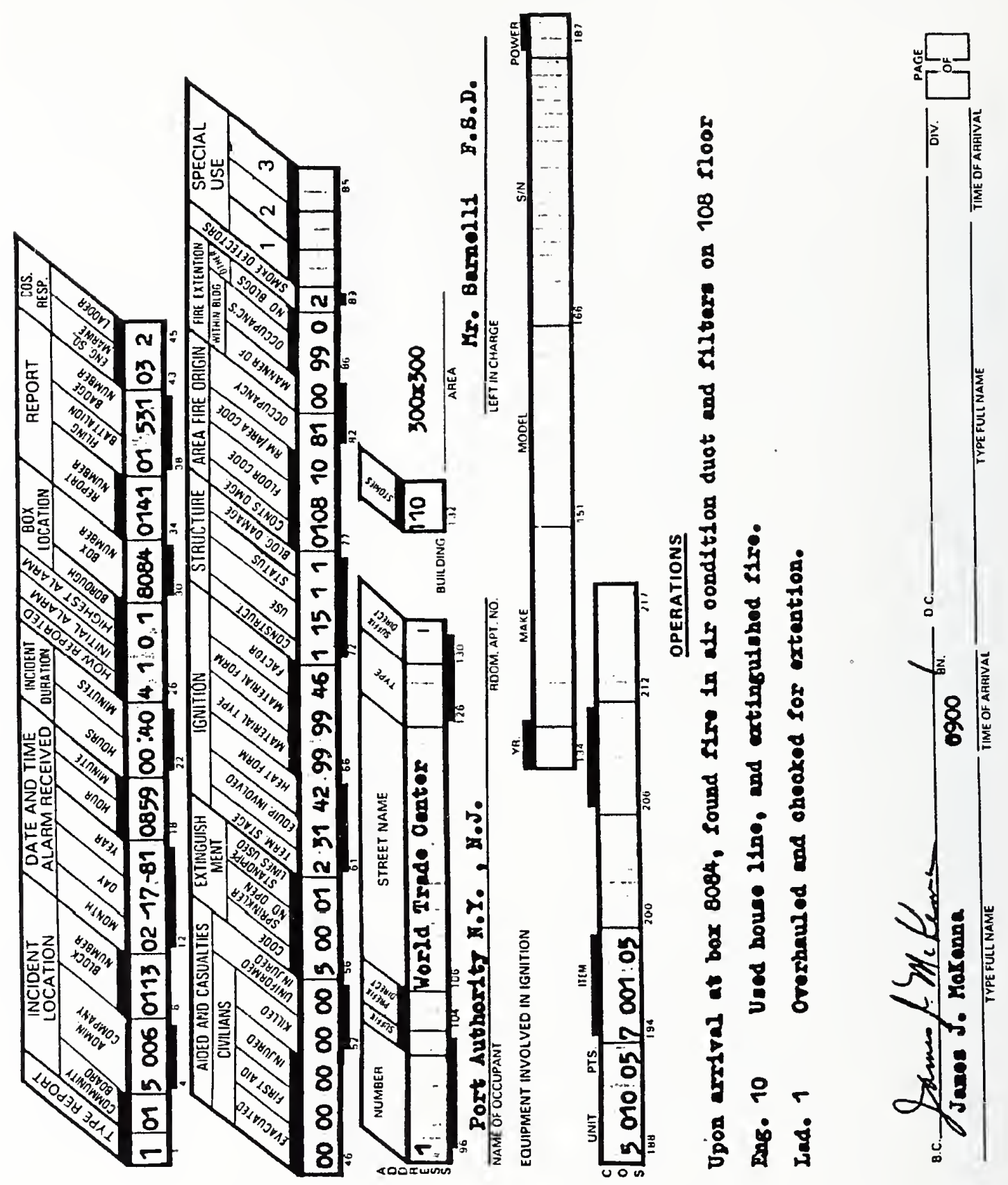




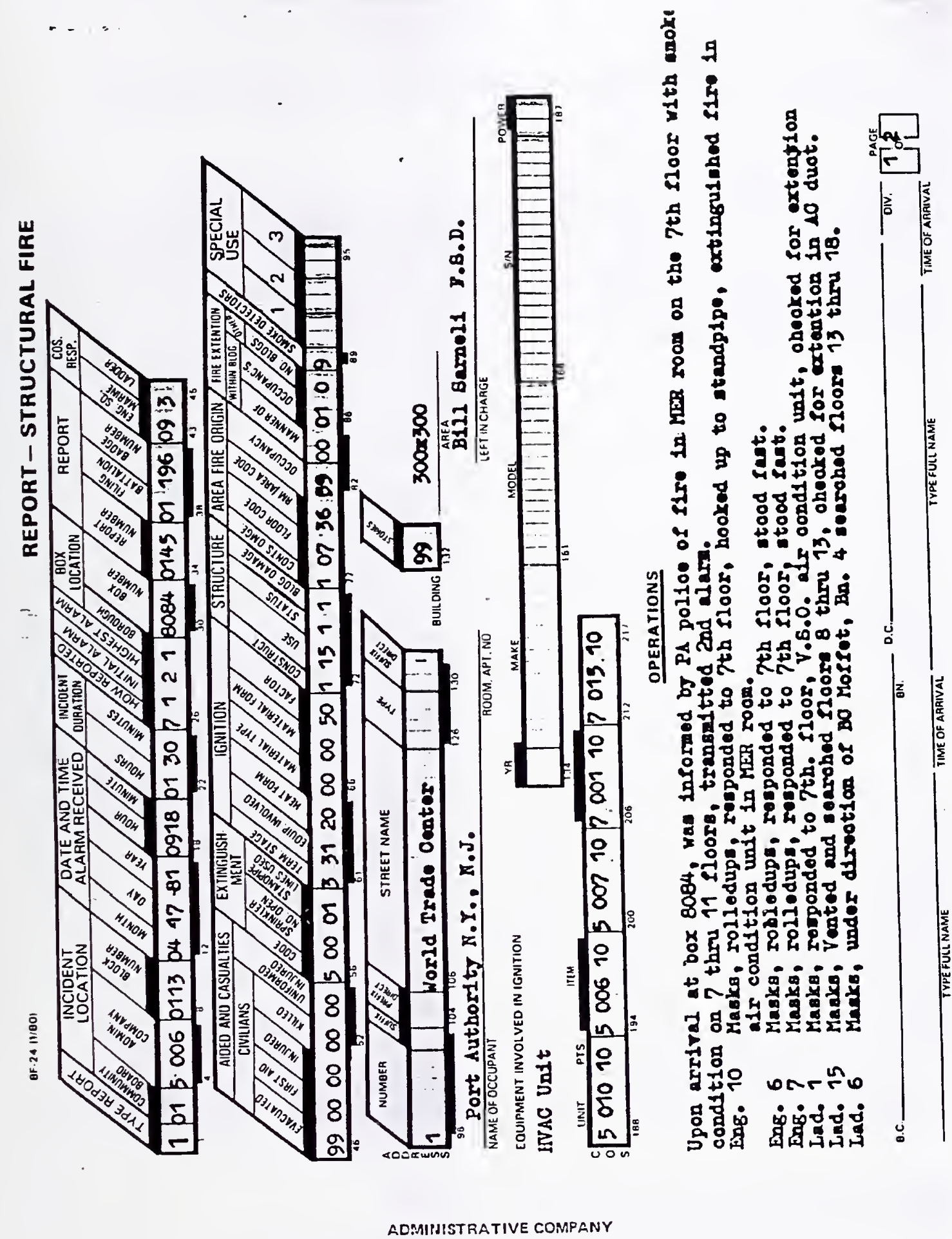




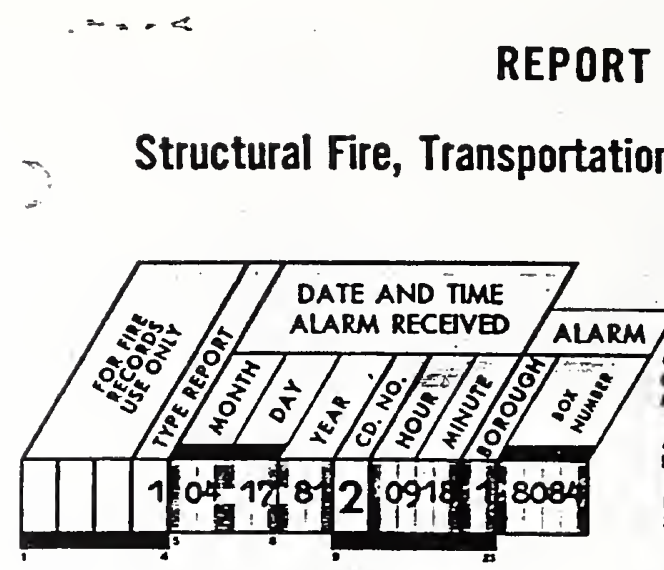

\section{REPORT - Additional Data}

Structural Fire, Transportation Fire, Non-Structural Fire or Emergency

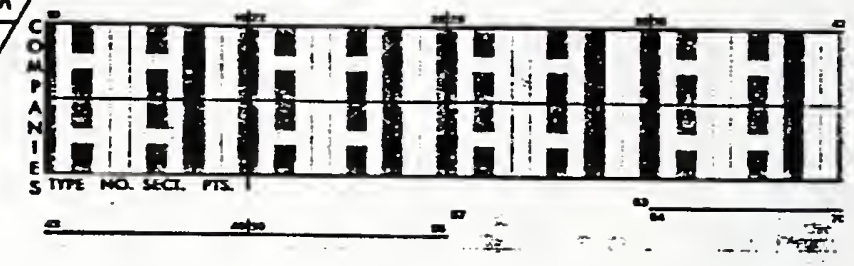

\section{OPERATIONS}

Rescue 1 Masks, searched floors 18 thru 23.

2nd. alam unta:

Eng8. 24, 15, 55,9, 241, and Karine 1 vere orded to atand fast at ther th assiened locations.

Div. 1, D.C. DeCaprlo responded and supervioed opperation: Chiefs Gormiey, Bishop, and Parrell also on sceno.

P.A. personell reported evacuation of aprax, 1,500 persons fron $100 r$

9 thru 23.

P.C. unit on scene

P.P. 2 on scene.

TIMES

\begin{tabular}{ll} 
& TIMES \\
\cline { 2 - 2 } Box & 0918 \\
$2-2$ & 0928 \\
U/C & 0959
\end{tabular}

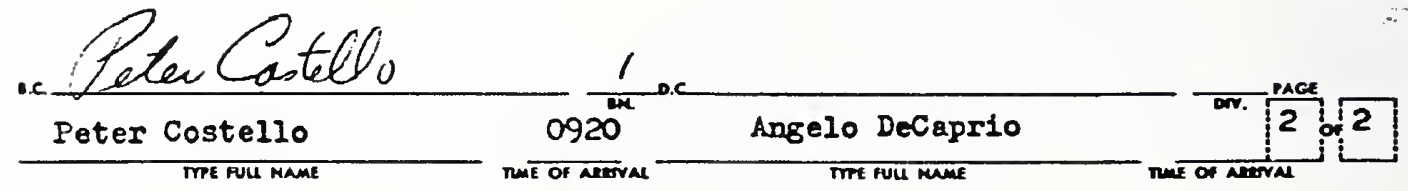

ADMINISTRATIVE COMPANY 


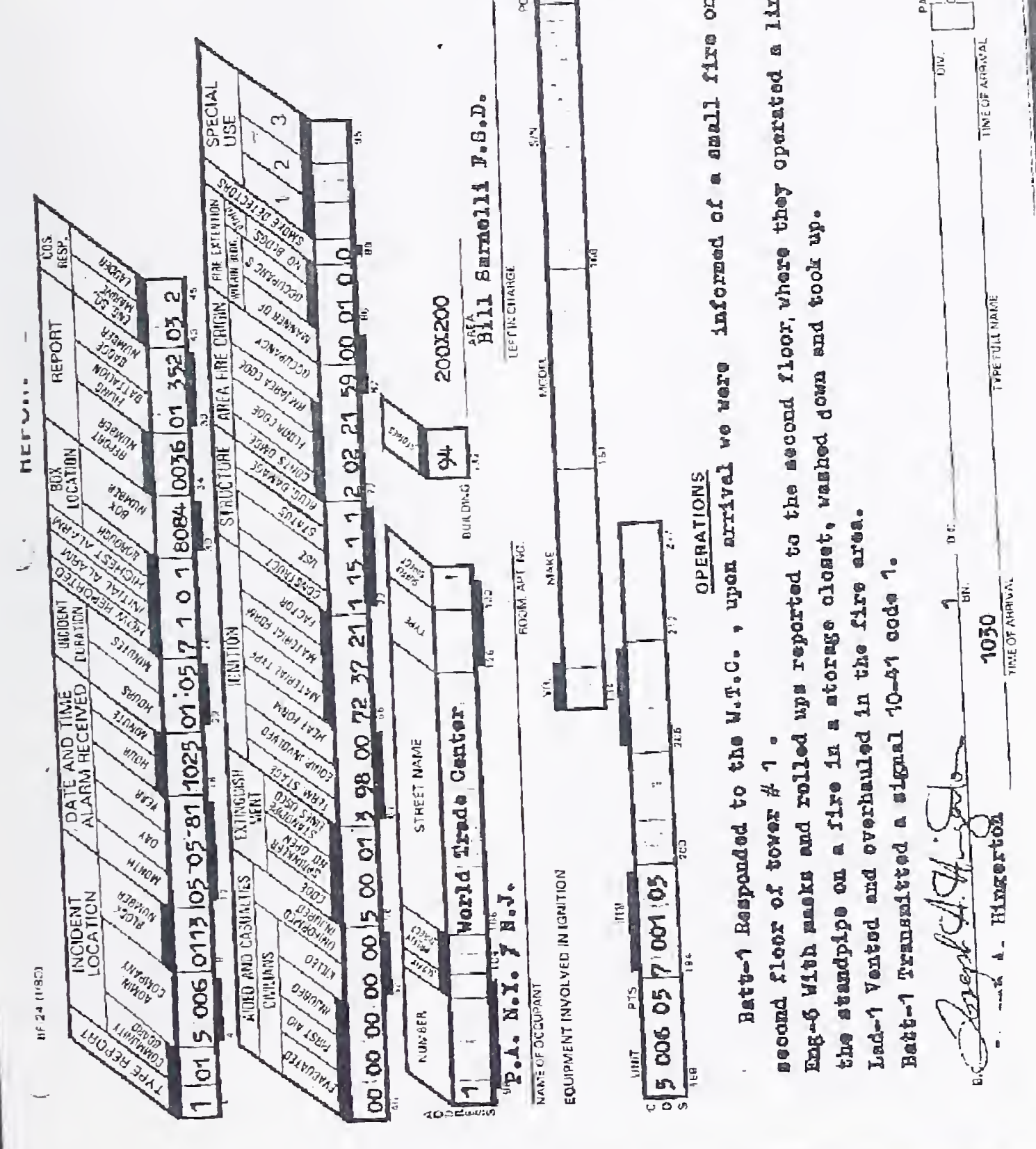




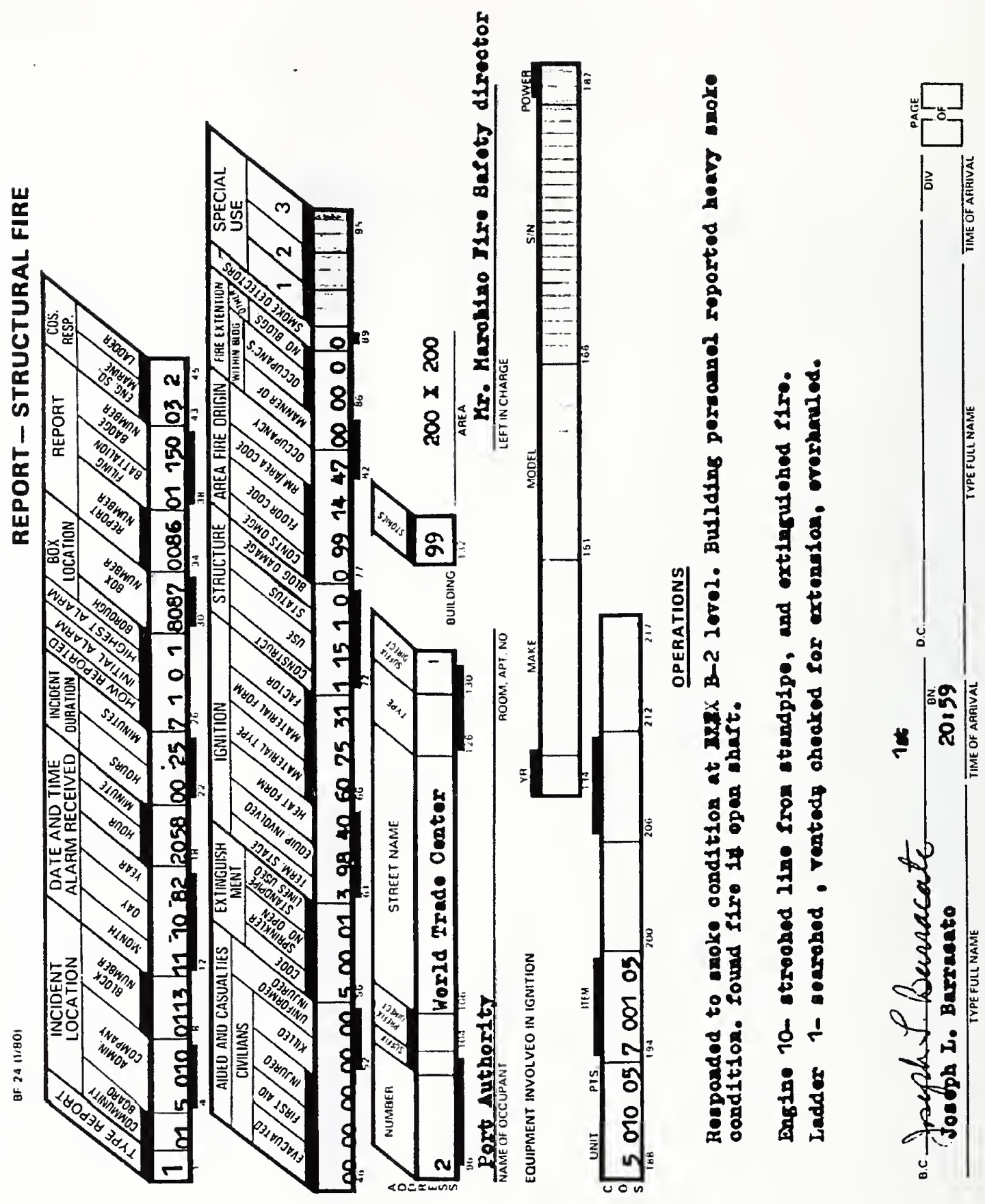

ADMUlviGTEATVE CORPANY 


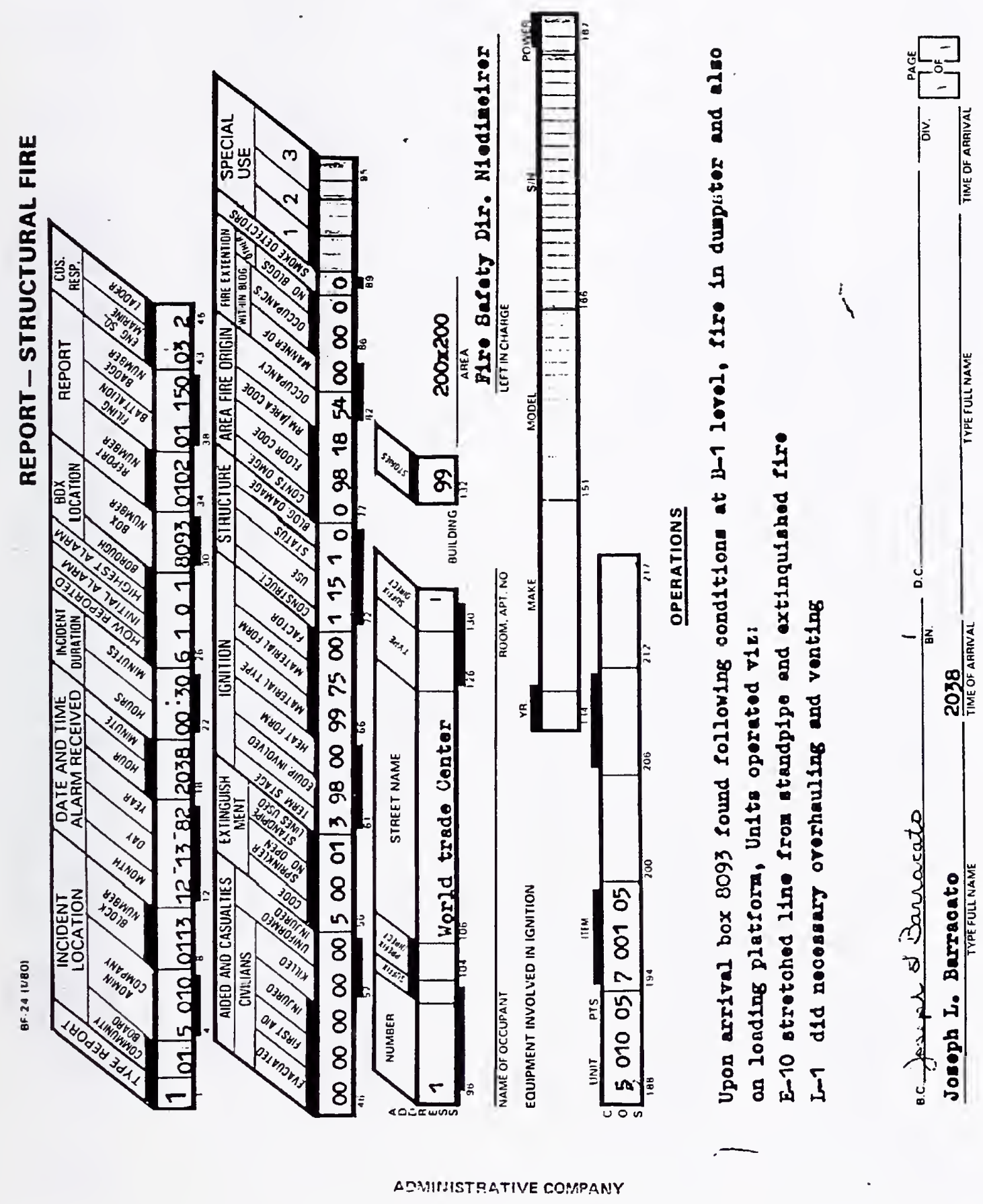




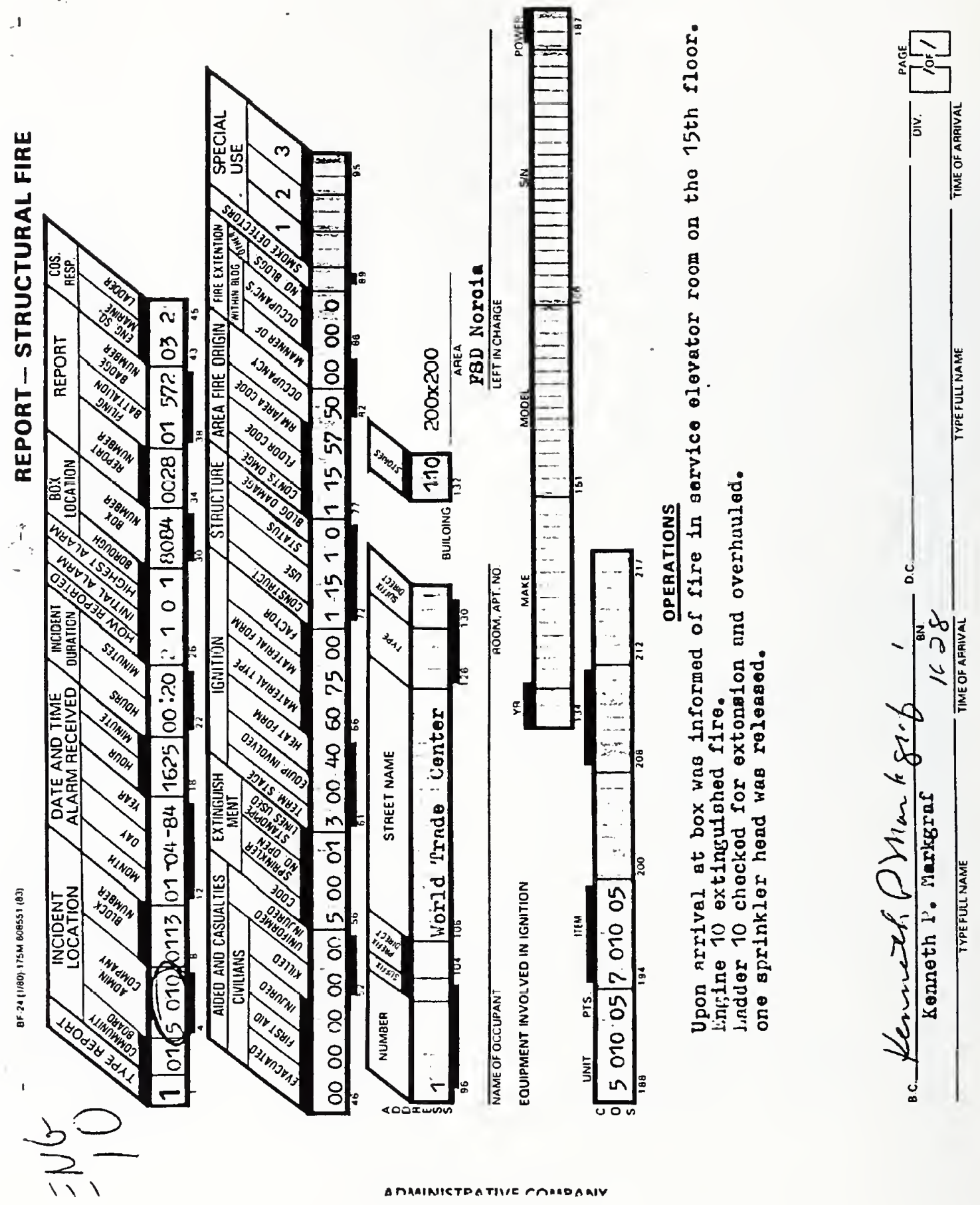




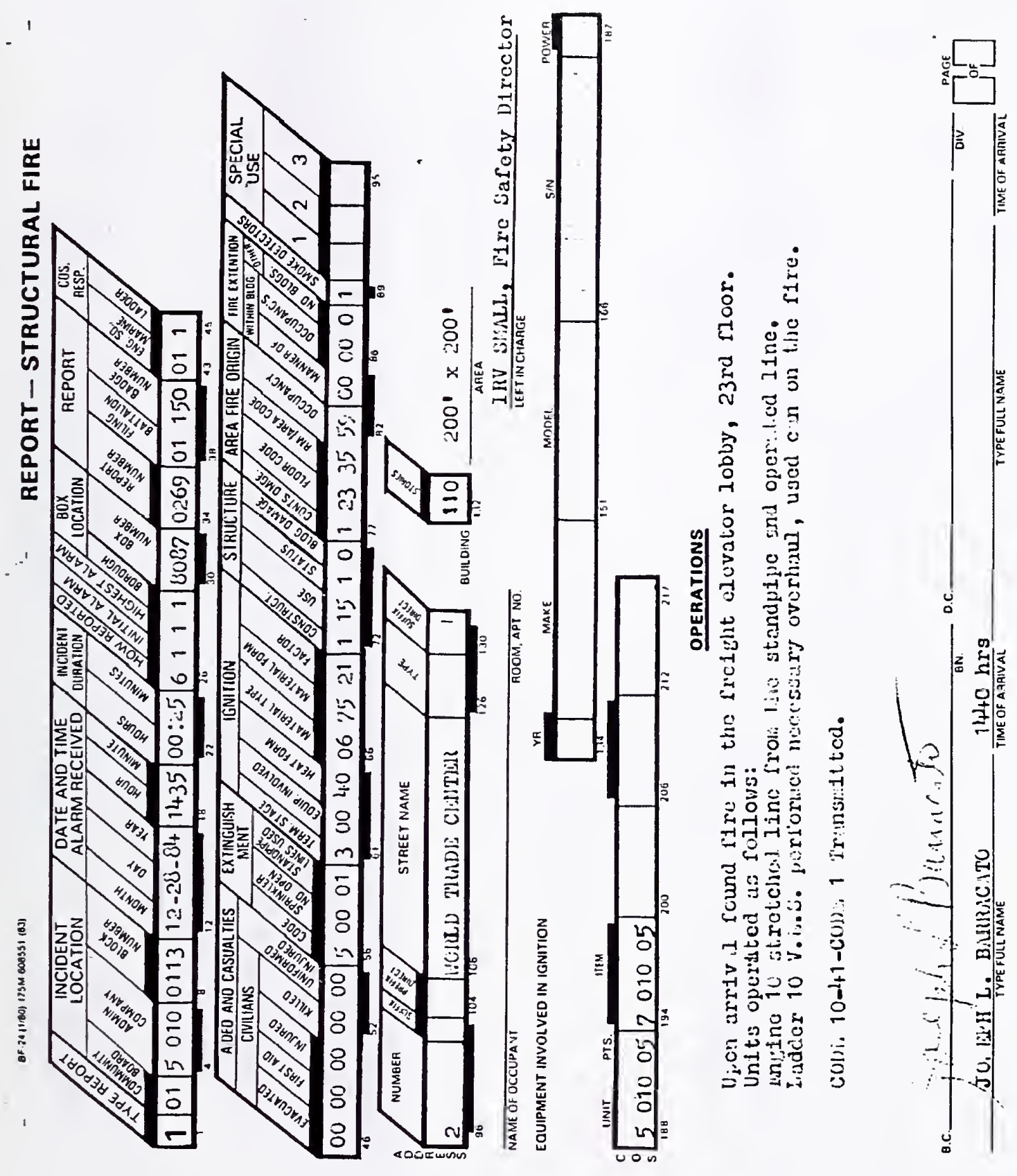




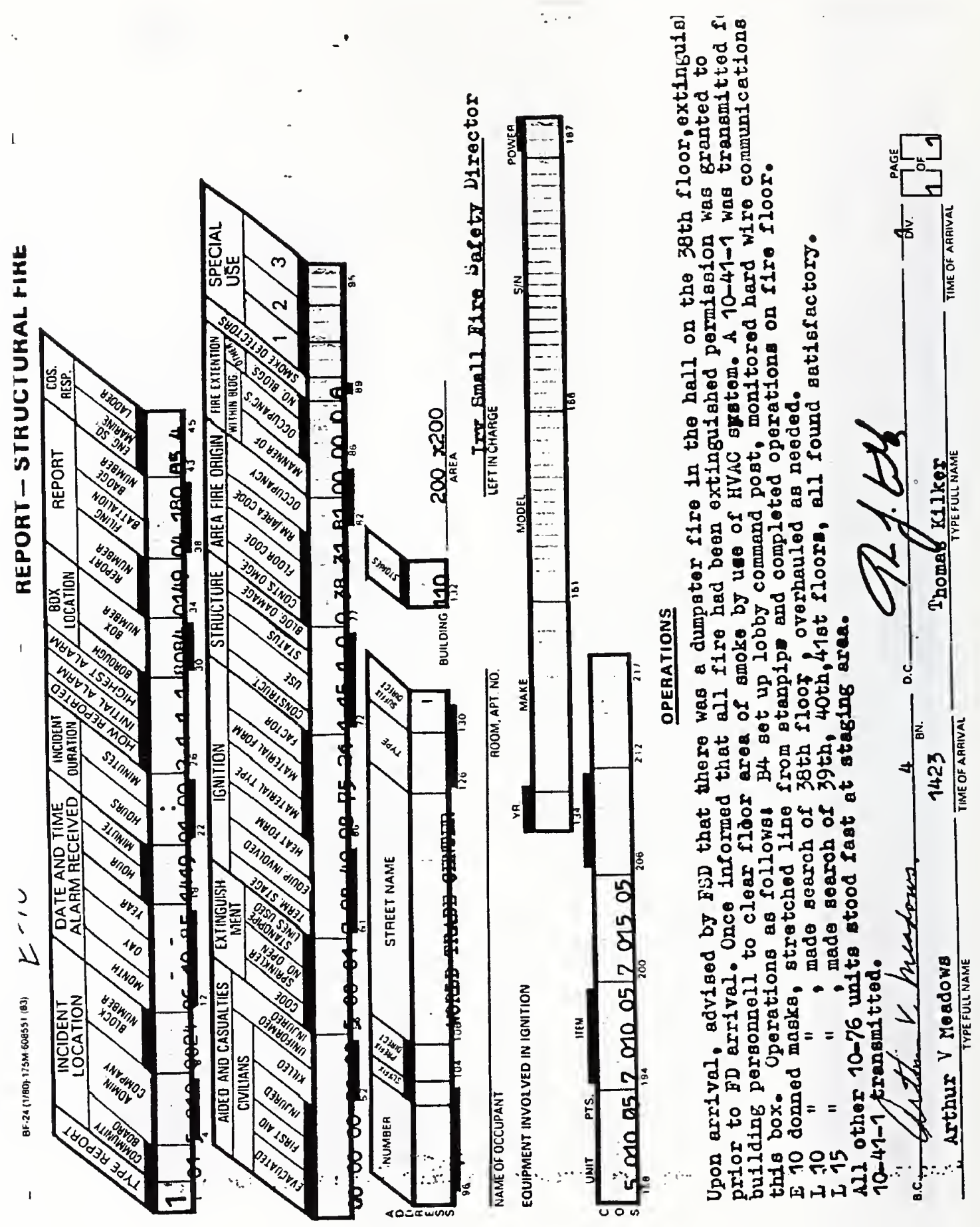




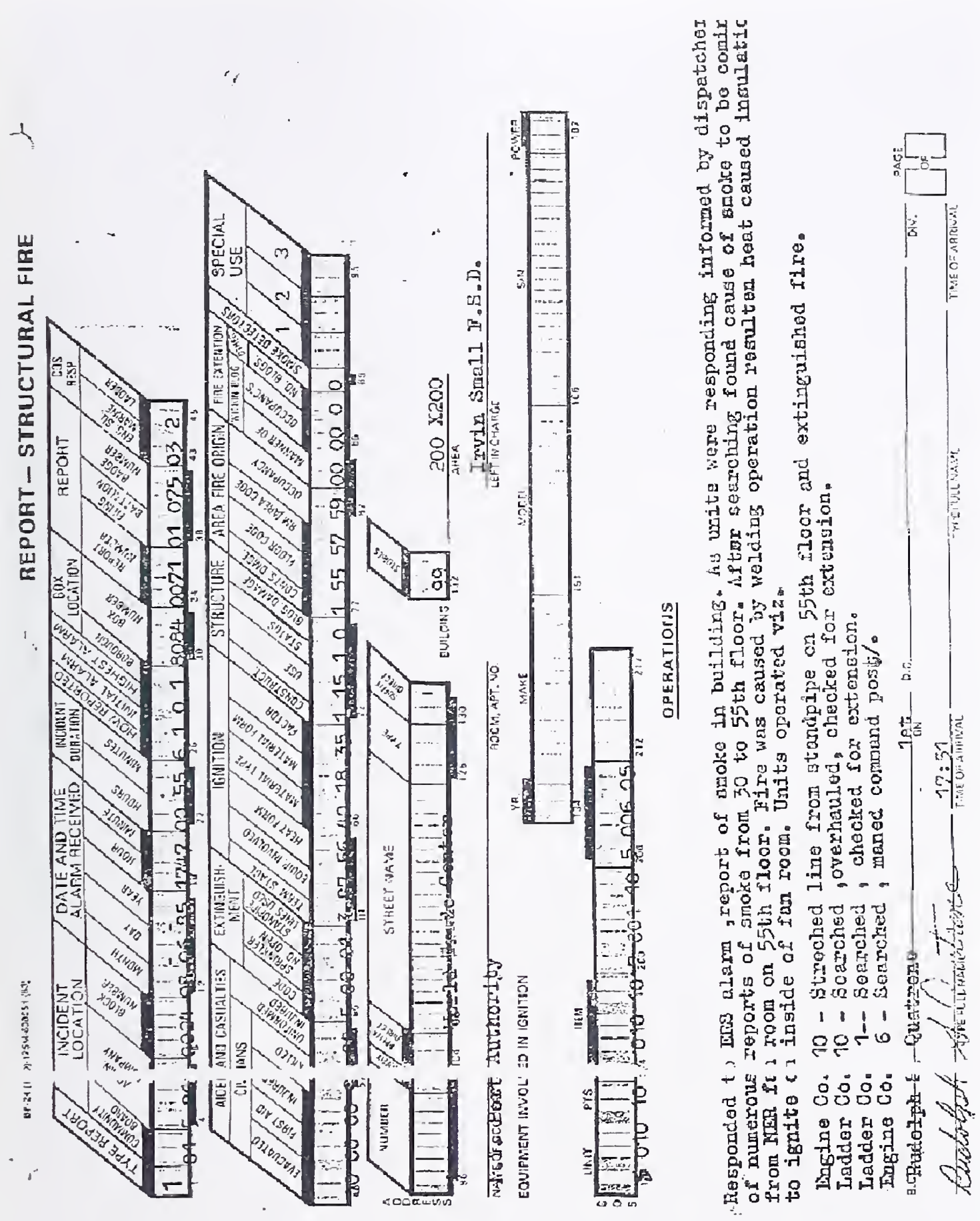

ADMINSTRATVE KOMPANY 


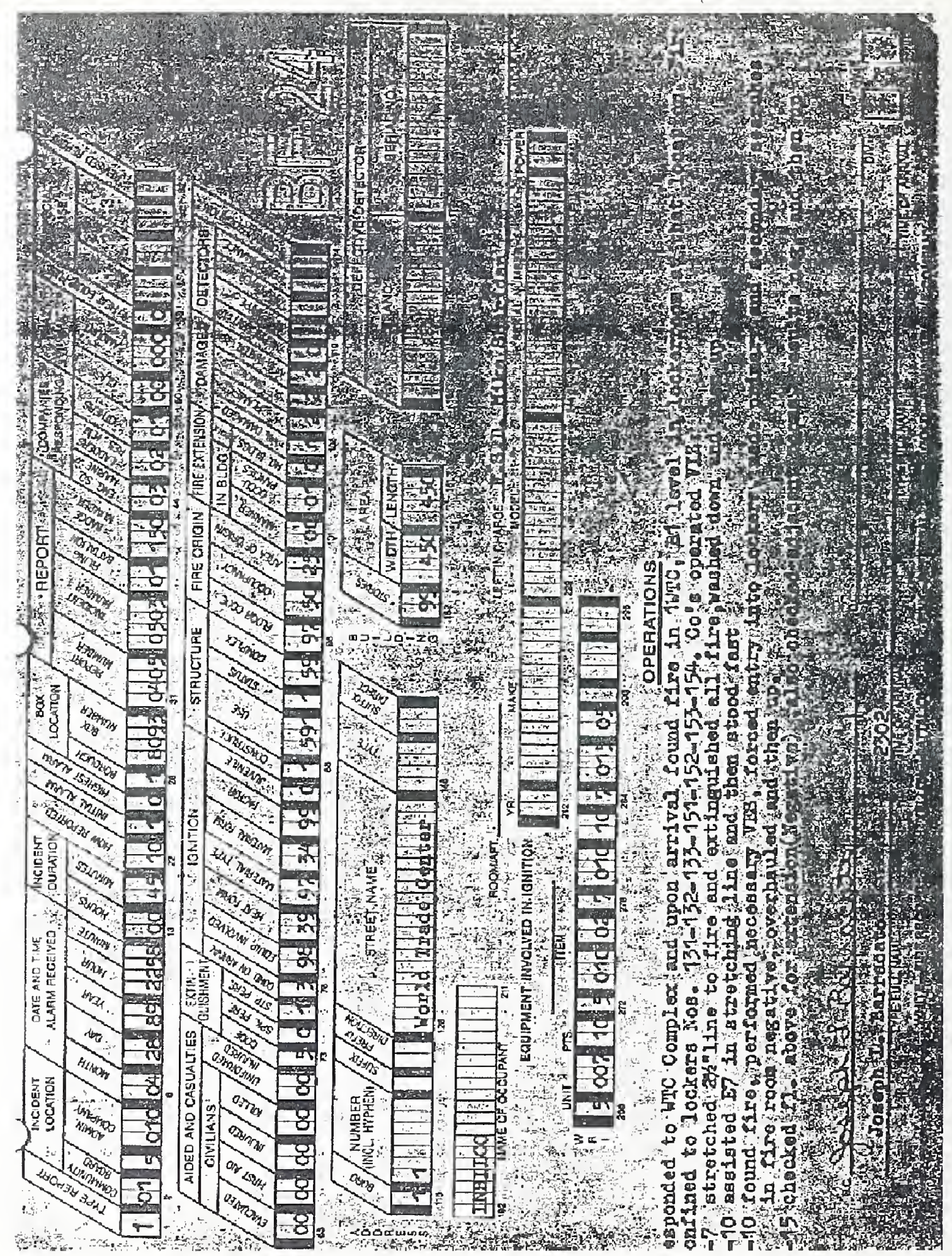




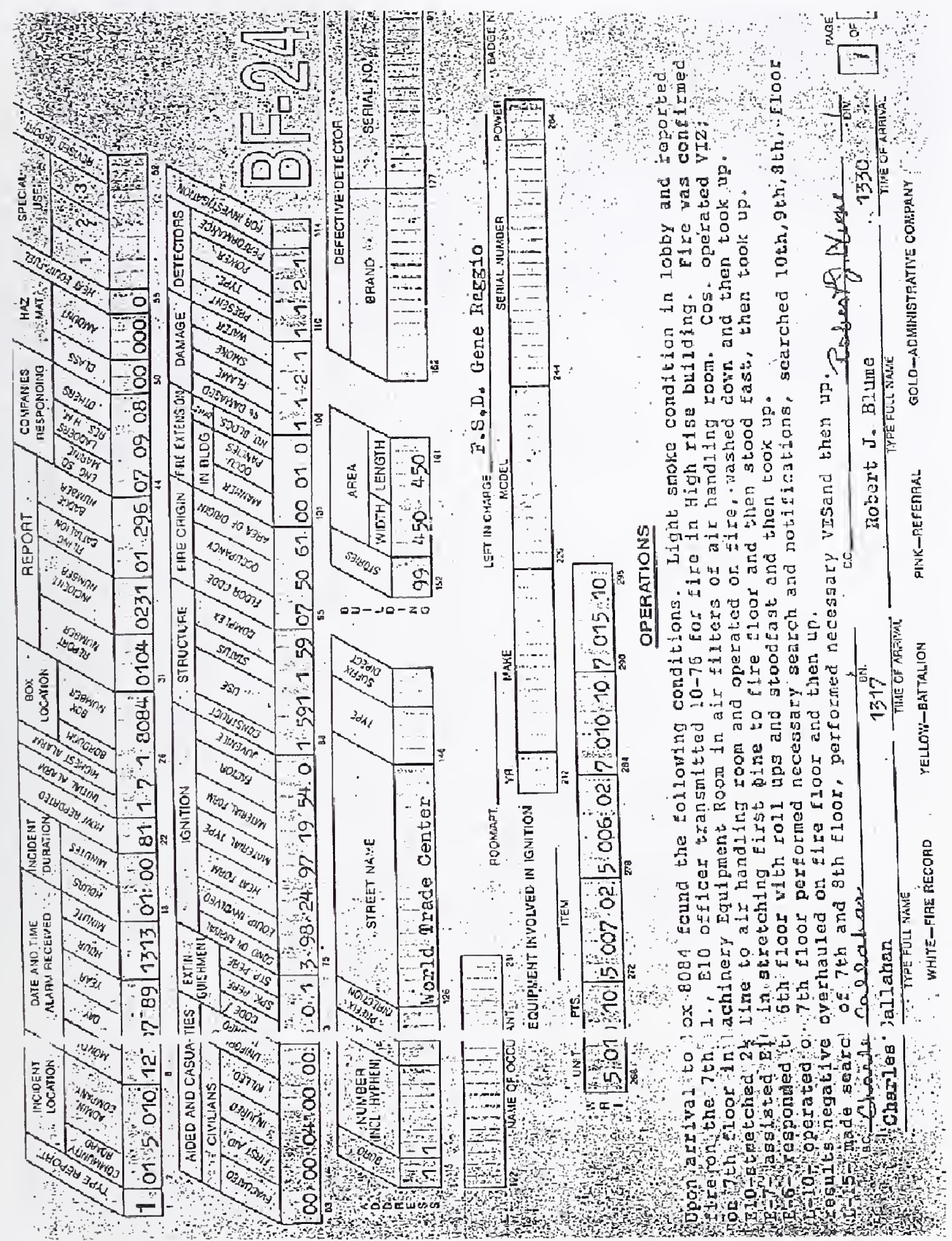




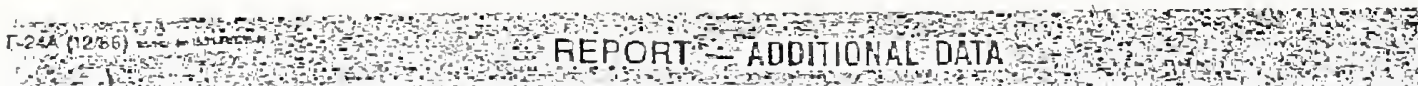
STRUCTURAL FIRE, TRALSPORTATIOH FIRE HON STRUCTURAL FIRE OR EMERGENCY.S
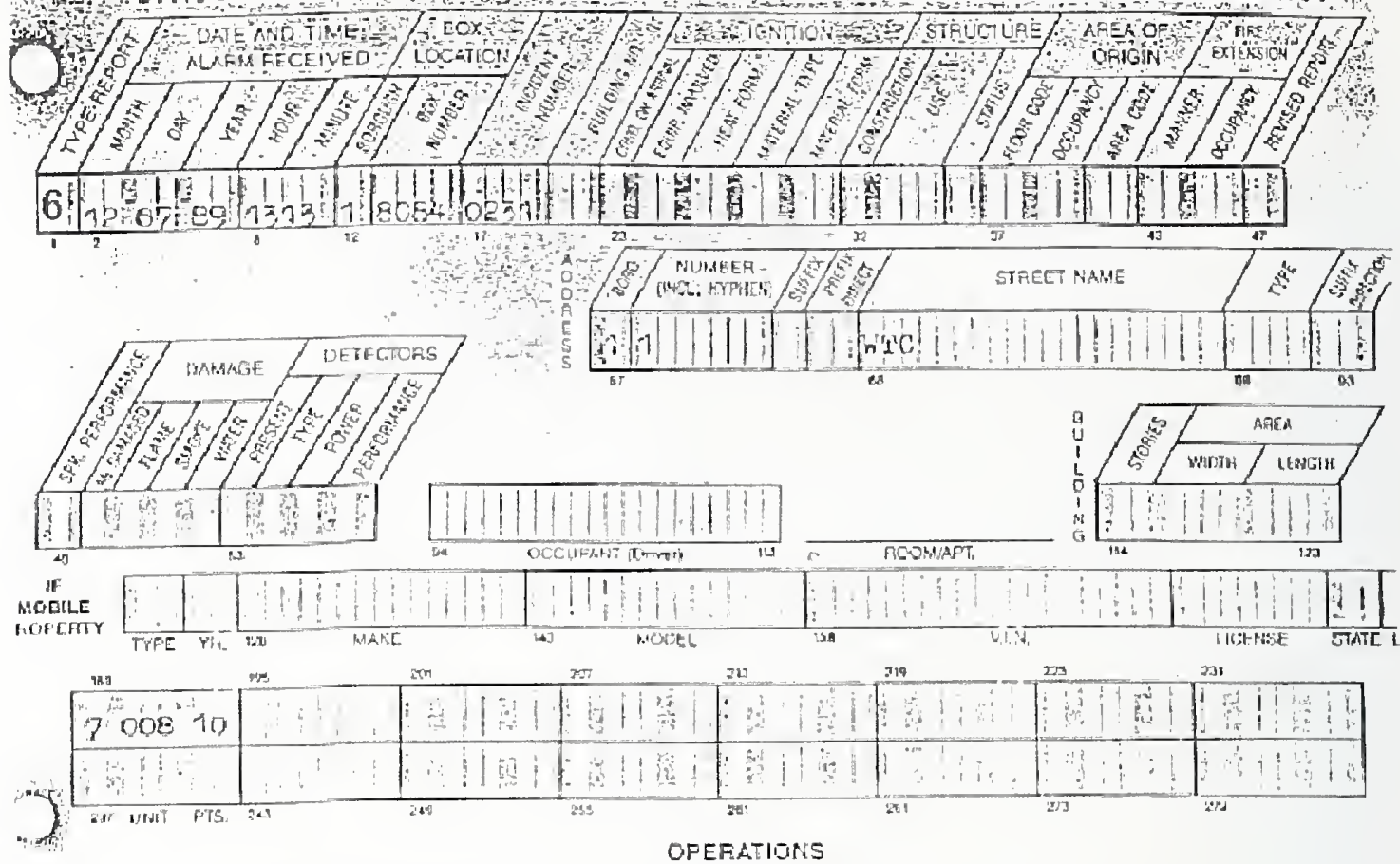

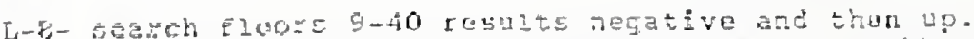

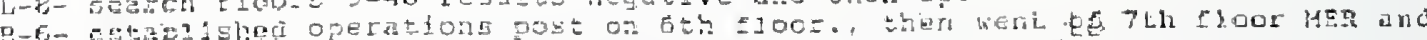

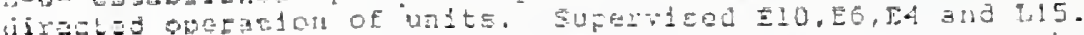

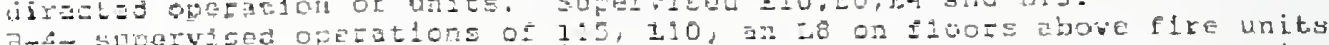

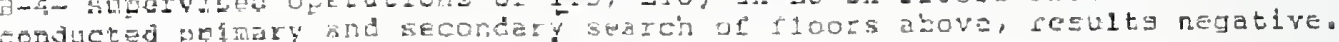
conduct

Tines 10-7e 1314- E10

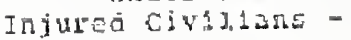

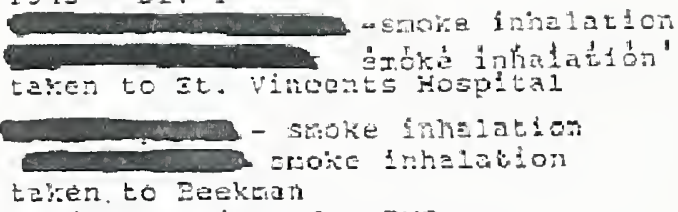

prs chief cervo fizected operations for sus

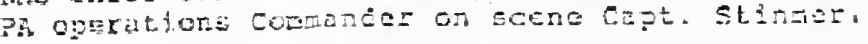

$\log _{10}$

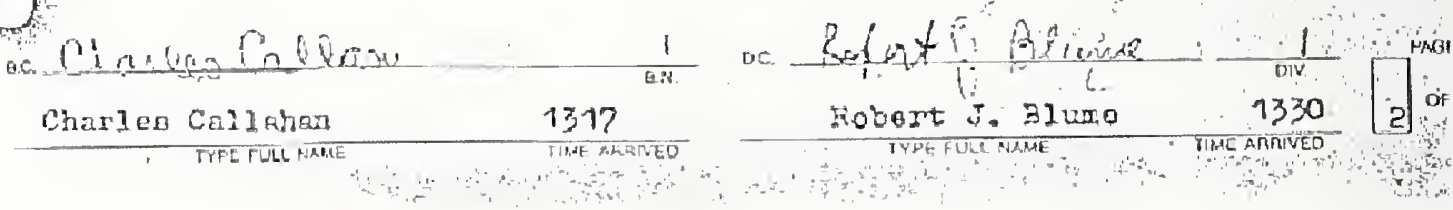




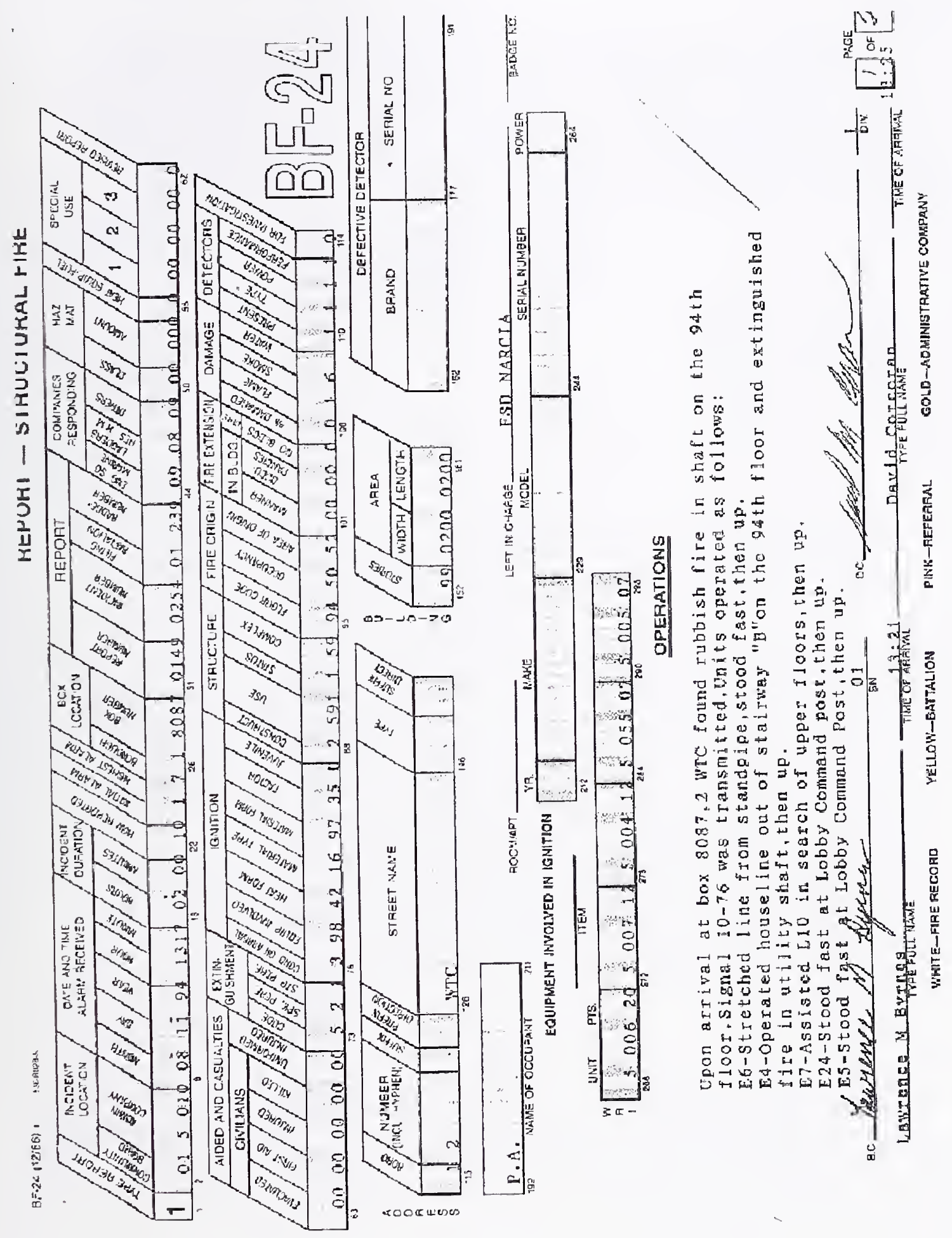


STRUCTURAL FIRE, TRANSPORTATION FIRE, NON-STRUCTURAL FIRE OR EMERGENCY
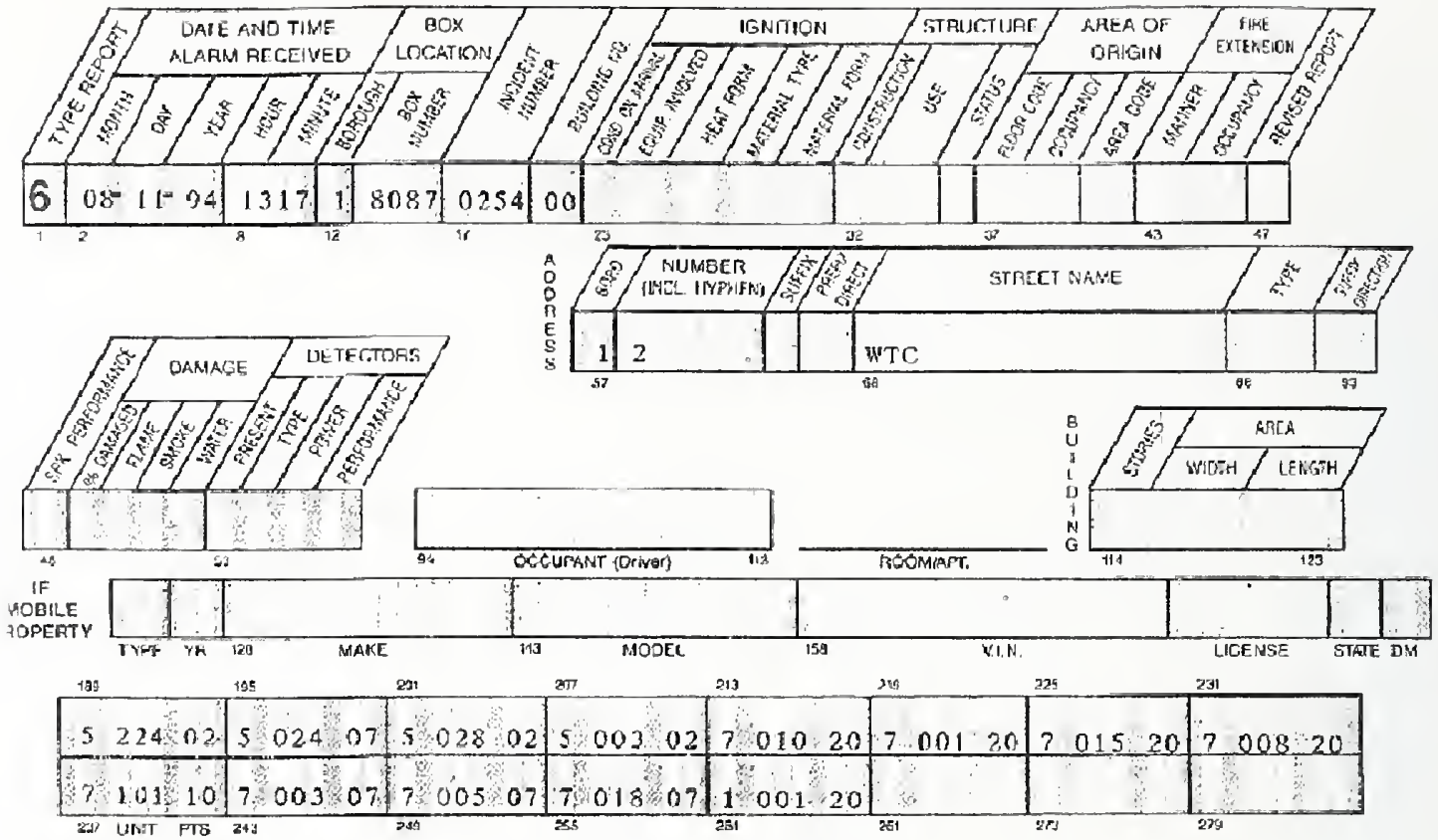

OPERATIONS

E. -Stood fiast at the lobby comara fost, then up.

E55-Stood fast al Lobby command post, then up.

I. I0-poeacted on the $107 \mathrm{th}$ floor, operad variuus walls and doors, finding no

fire or extention, then up.

L. 15 -Assisted $R 1$ ir opening up a wall exposing fire is shart, made necessary search and exam from 44 thru 99 th floorllermigation point of

shaft iNcgntive.

L8-Kade aecessary search and exam of floors 100 thru 106. found negatinc.

1.5-5tood fast at the Lobby command Fost, then up.

1.18-5tood fast at the lobby command post, then up

L.101-Seajsh 75,91 and 92nd floor, found negative, ihen up.

I. - Made recessary seatch and cxam of floors 104 thru log,then up.

L224-stood fast at the Lubby command Post, then up.

R1-lsed Thermal-Imaging camera and located i ire in shaft on the 94 th floor.

Betta-supervised operation on the fire floor,then up.

hitti-supervised operations on 107 and 74 fluots, then up.

ball 2-stood fast, thon up.

FCU-Monilored handi-lalkie ciscuit ptogress reports, then up. A

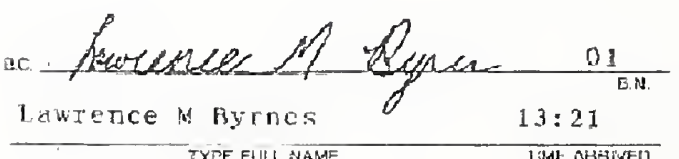

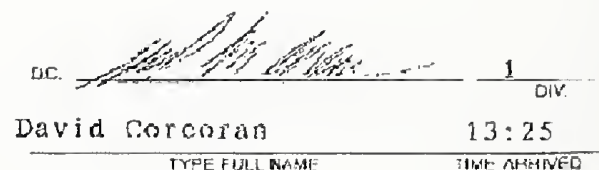

of or 


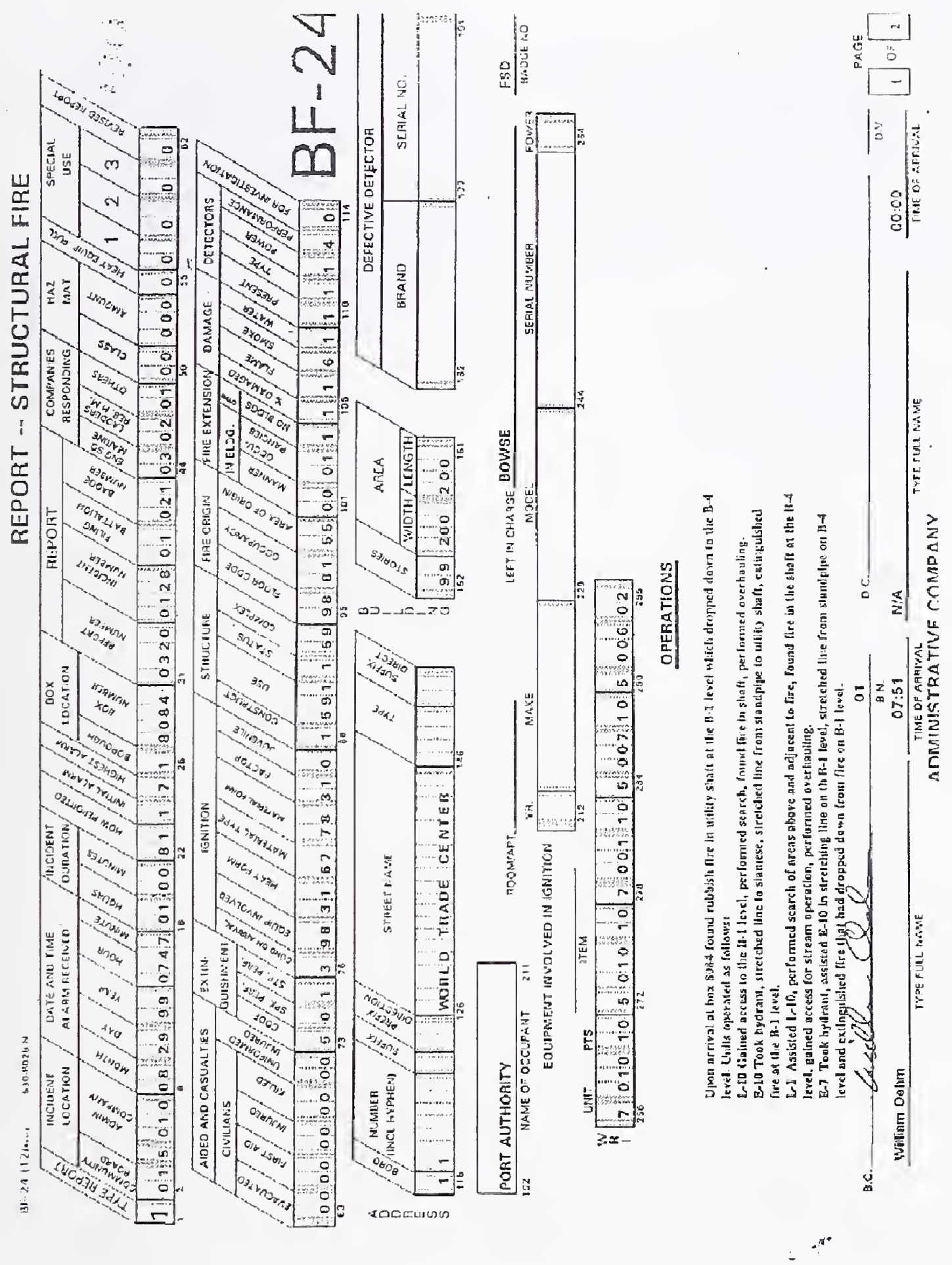


STRUCTURAL FIRE, TFANSPORTATION FIRE, NON-STRUCTURAL FIRE OR EMERGENCY
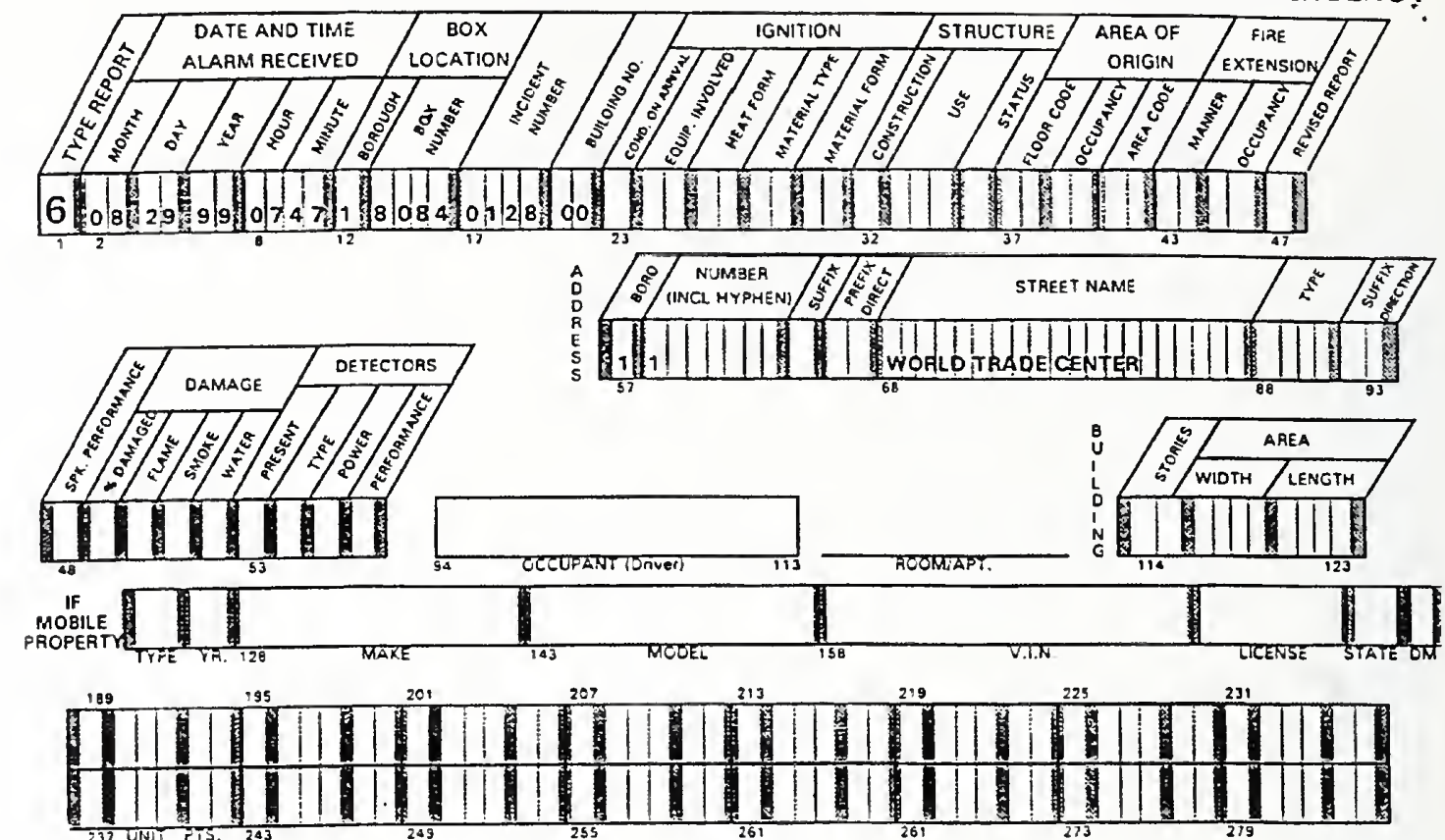

OPERATIONS

E-6 Took bydrant, 25sisted E-7 in stretch.
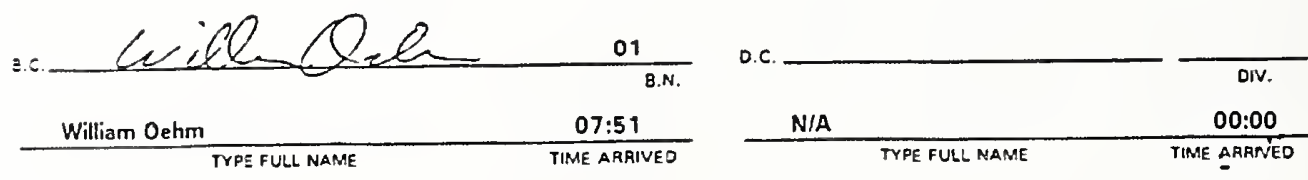

2 PAGE 


\section{Attachment G-A.7}

Significant fires occurring in WTC 7 (1)

\begin{tabular}{|c|c|c|c|c|c|c|}
\hline $\begin{array}{c}\text { Significant } \\
\text { Fire }\end{array}$ & $\begin{array}{c}\text { Incident } \\
\text { Date }\end{array}$ & Fire Location & $\begin{array}{c}\text { \# sprinklers } \\
\text { activated }\end{array}$ & $\begin{array}{c}\text { \# standpipes } \\
\text { activated }\end{array}$ & $\begin{array}{c}\text { Cause of } \\
\text { fire }\end{array}$ & $\begin{array}{c}\text { Material } \\
\text { Ignited }\end{array}$ \\
\hline 1 & $5 / 20 / 88$ & $\begin{array}{c}\text { Construction } \\
\text { shanties on floor } \\
3\end{array}$ & $\begin{array}{c}\text { Multiple, \# not } \\
\text { listed }\end{array}$ & 1 & Suspicious & Shanties \\
\hline
\end{tabular}




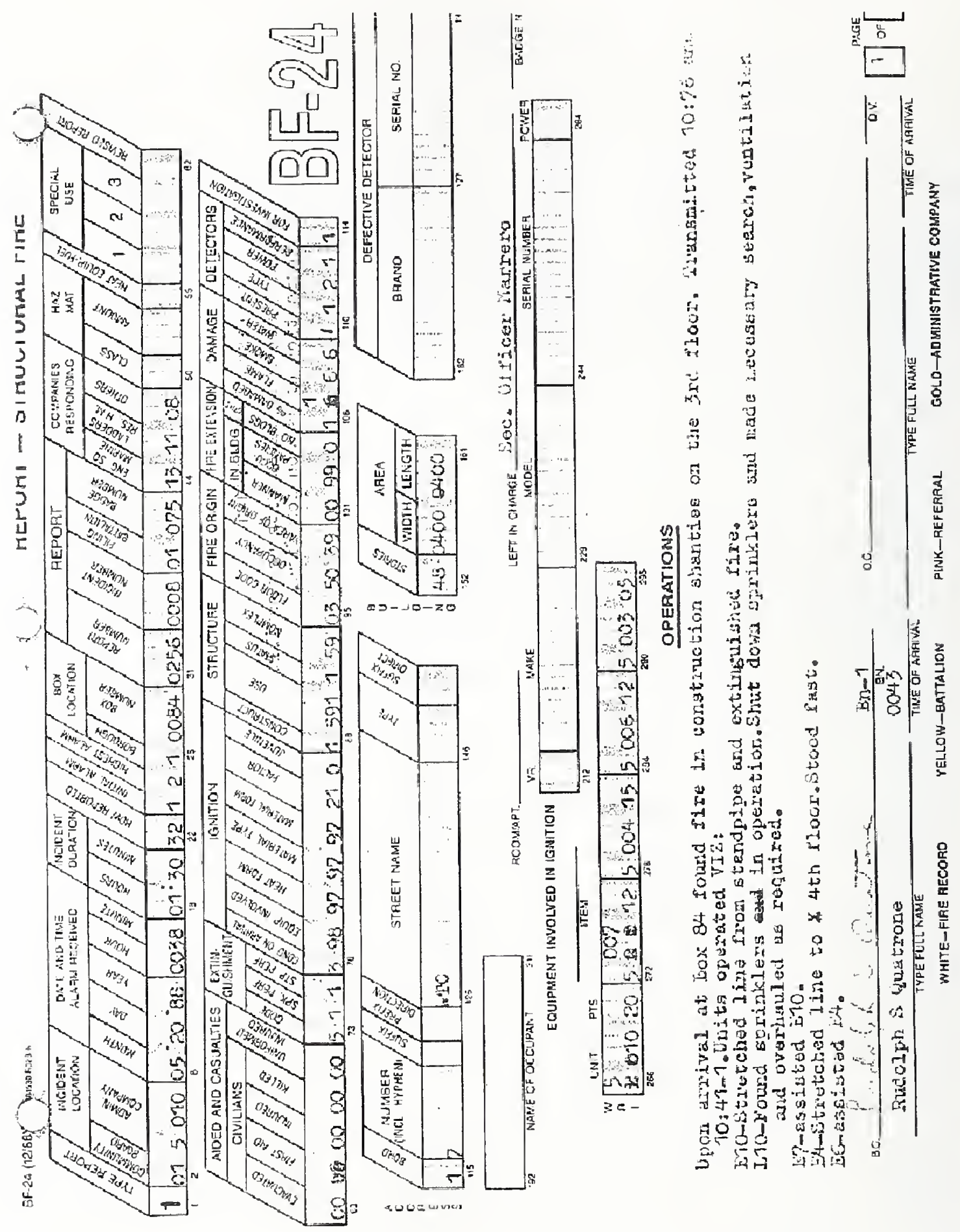




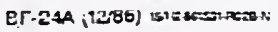

REPORT - ADDITIONAL DATA

STRUCTURAL FIRE, TRANSPORTATION FIRE, NON-STRUCTURAL FIRE OR EMERGENCY
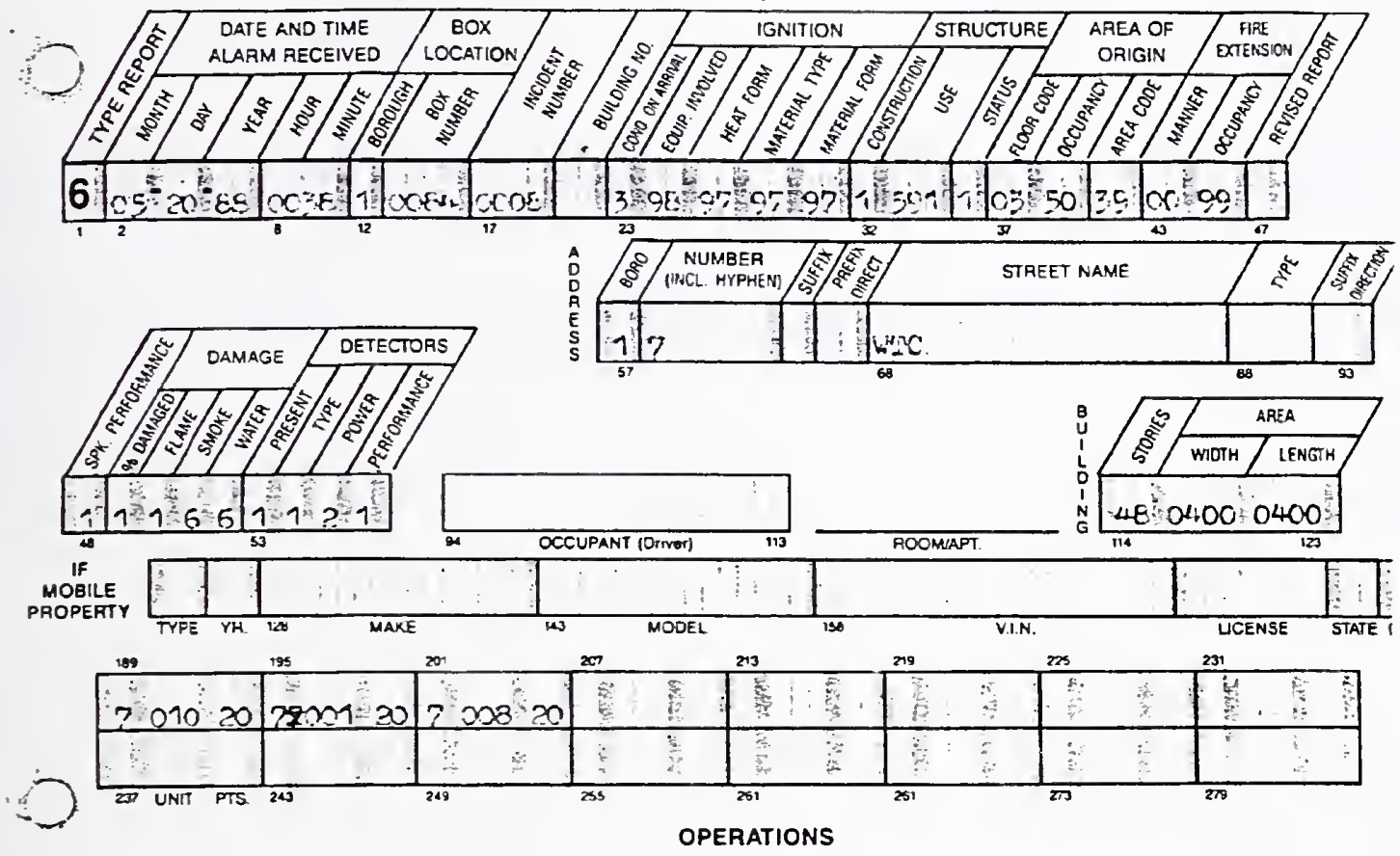

Operations as followi;

is-hasisted I10 ilade primary and secendory search of 5-10,11,12. Then up. If liace search and exerination of the 4th rloor. Then assisted is ir the exainstion c? licper floors. Then up.

Eatt 4 Supervised ETC and 210 on fire floor.

Batt 2-Supervised 54 asd $\bar{E}$ and L1 on floors ebove sire.

FCU- SOF

MSU-SCP

E3- Stood fast.

Bett 6- Stocd fest.

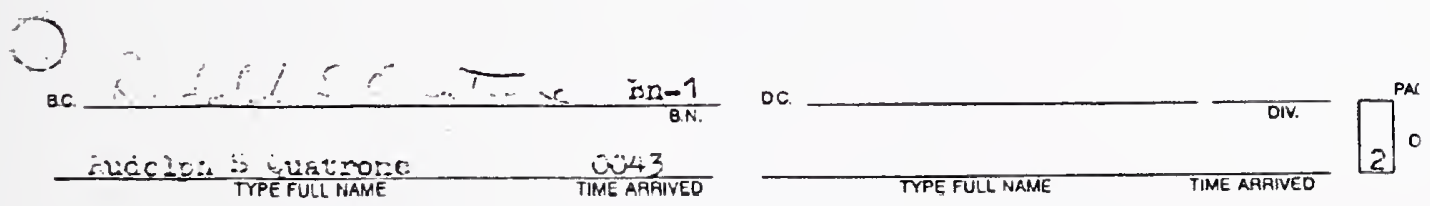


This page intentionally left blank 


\section{Attachment G-B}

PACO 2002 Report: World Trade Center General Description of All Building Systems and the Capital Program. Extracted page.

Miscellaneous Life Safety Improvements and Sprinklerization Program 
12. Miscellaneous Life Safety Improvements and Sprinklerization Program

\subsection{Description of the Program}

The initial base building provided for Fire Standpipe (FSP) protection in the Towers and Plaza Buildings and no sprinkler system installation (except in the sub-grade Levels). In response to the enactment of Local Law 5 and other NYC Bullding Code Local Law enactments related to the fire protection of high rise office towers buift after 1973 the Port Authority voluntarily retrofitted the WTC complex to comply with the new NYC Building Code Requirements supplemented by PA imposed life safety requirements unique to the WTC. These requirements provided tenants the option of achieving fire protection compliance by a compartmentalization or a sprinklerization option. The Scope of miscellaneous life safety and sprinkler system upgrades/improvements, other than certain fire alarm, concourse circulation and blast recovery improvements discussed elsewhere, included but was not llmited to:

\subsection{Scope of Fire Life Safety Improvements}

\section{Architectural Modifications - On all floors:}

- Upgraded core wall construction to provide for a 2 hour rated fire separation from one side of a tower floor to the opposite side (Where core wall were already 2 hour rated, such as in elevator shafts, upgraded where not previously required).

- Installed a double acting set of rated HM fire doors in the core corridors where the above 2 hour rated fire separation crossed the corridor (This enabled one half of a typical tower floor to serve as a horizontal fire refuge for the other.) Doors included special magnetic hold open hardware and closures linked to smoke detectors in floor return air ducts.

- Restored all ceilings in corridors and lobbies affected by fire protection Installations.

- Provided slab-to-slab thour fire rated walls to enable a maximum of 10,000 gross square foot compartmentalization on each side of the 2 hour rated fire separations.

Sprinkler System Installation

- The entire WTC complex was fuily sprinklered. The Sprinkler System was Installed in three basic phases. Phase 1: Sub-grade areas for the Initial building consfruction; Phase 2: Sprinkler riser/main installation throughout 1 \& 2 WTC including the sprinklenization of corridors, storage rooms, lobbies and certain tenanUPA spaces in 1976 in compliance with Local Law 5 to provide tenants with a compartmentation or sprinkler fire protection option; Phase 3: The full sprinklerization of the entire complex for all remalning places from 1983 to the 2001 (including 1993 blast recovery and ongoing up-grades/improvements and replacements).

- The Tower sprinkler systems were fed in the various zones from the gravity feed fire reserve tanks located on the $20^{\text {th }}, 41^{\text {st }}, 75^{\text {th }}$ and $110^{\text {th }}$ Floors through a $4^{\text {" }}$ vertical sprinkler riser located in the Janitor Closet on each floor of each building in the WTC complex. 


\section{TABLE OF CONTENTS}

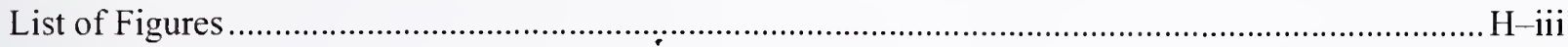

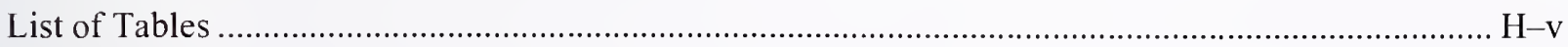

\section{Appendix $\mathrm{H}$}

Interim Report on Evolution of WTC Fires, Smoke, and Damage Based on

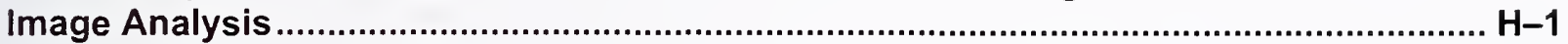

H.1 Collection and Analysis of Visual Material................................................................... H-1

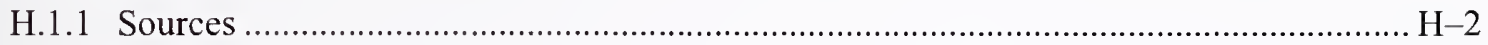

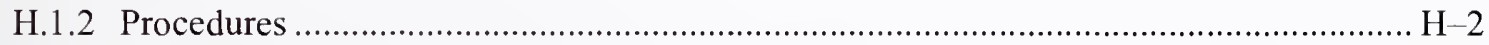

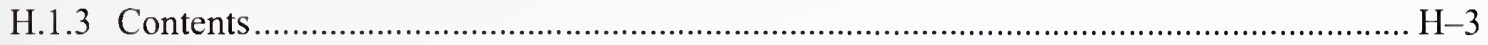

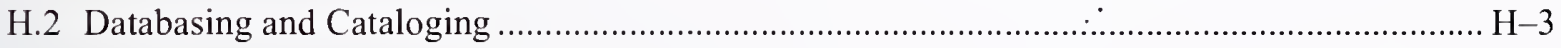

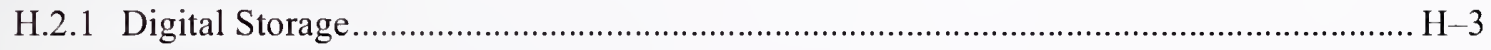

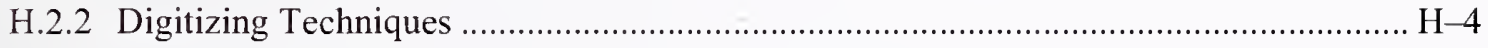

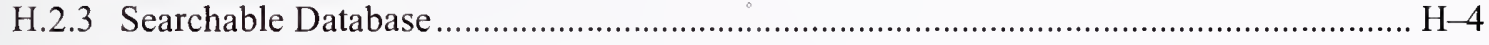

H.3 Timing of Photographs and Video Clips......................................................................... H-8

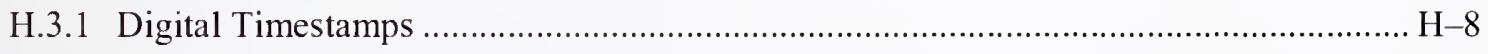

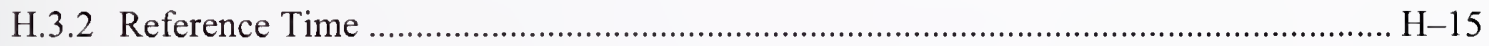

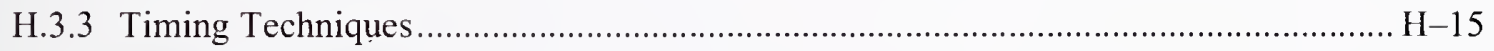

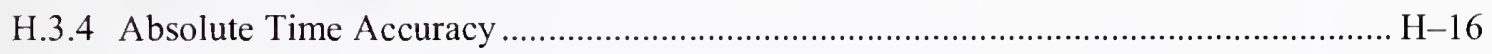

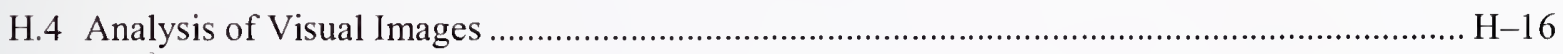

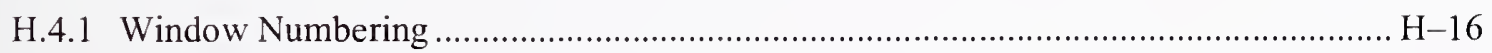

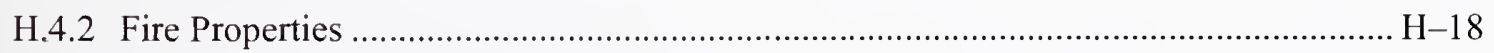

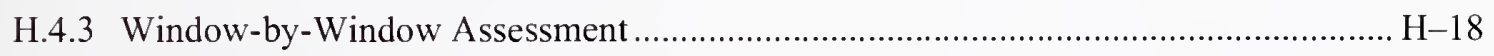

H.5 Initial Damage Patterns on WTC 1 and WTC 2 Due to the Plane Strikes ................................ H-22

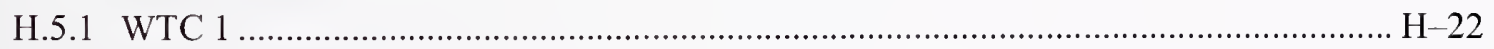

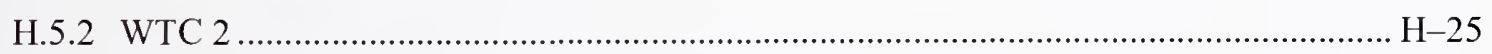

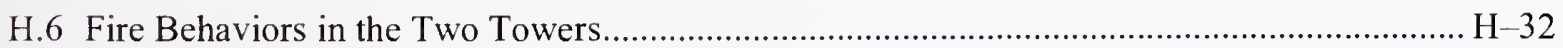

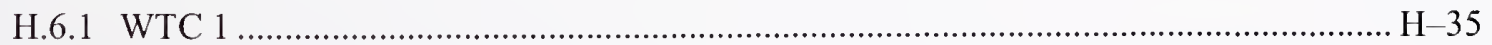

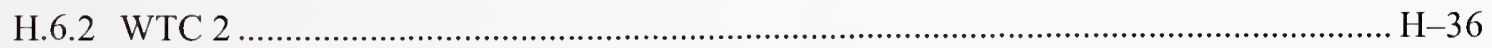

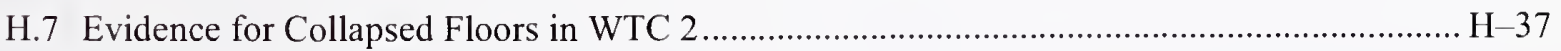

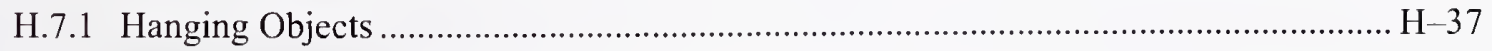

H.7.2 Molten Material .................................................................................................. H-38 
H.8 Progress on Collection of Images and Analysis for WTC 7

H-39

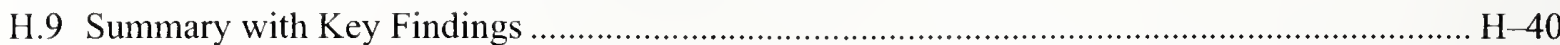

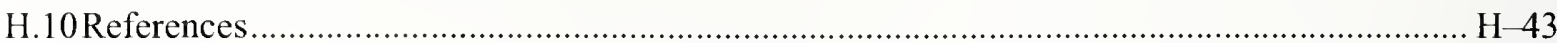




\section{LIST OF FIGURES}

Figure H-1. An example of the VideoList data entry sheet for video assets.

H-5

Figure $\mathrm{H}-2$. An example of the first page of the Cumulus data entry sheet for photographic assets.. H-12

Figure $\mathrm{H}-3$. An example of a Cumulus asset screen display for the video database

$\mathrm{H}-12$

Figure $\mathrm{H}-4$. An example of the PhotoTiming sheet for calculating times for photographs containing Exif meta data......

Figure $\mathrm{H}-5$. An example of the VideoList sheet for calculating clip times for video assets.

H-14

Figure H-6. Photograph taken at 9:26:20 a.m. on September 11, 2001, showing the east face of WTC 2

Figure $\mathrm{H}-7$. This photograph is cropped from the image shown in Figure H-6. It was taken on September 11 and shows the east face of WTC 2 at the northeast corner from floor 77 to floor 82 .......

Figure $\mathrm{H}-8$. The key used to describe observations with regard to fire, smoke, and window breakage in Excel data files for individual windows in the two towers.

Figure H-9. A portion of the Excel spreadsheet describing fires on the east face of WTC 1 around 9:42 a.m.

Figure $\mathrm{H}-10$. A representation of fires for floors 91 to 100 on the east face of WTC 1 around 9:42 a.m.

Figure $\mathrm{H}-11$. A single frame from a time-dependent visualization generated by Smokeview .

Figure $\mathrm{H}-12$. A drawing of the damage to the steel façade of WTC 1

Figure $\mathrm{H}-13$. The condition of windows is shown for the four faces of WTC 1 around 8:47 a.m. shortly after it was struck by American Airlines Flight 11

Figure $\mathrm{H}-14$. Photograph showing a full panel section lying in Cedar Street near its intersection with West Street

Figure H-15. Series of sequential cropped frames taken from a video shot on September 11, 2001, showing the plane approaching WTC 2

Figure $\mathrm{H}-16$. Plots of pixel locations for the nose and tail of the plane that struck WTC 2 as a function of time.

Figure $\mathrm{H}-17$. Frames from a composite video

Figure $\mathrm{H}-18$. The time when null points are observed in the difference video following the plane strike on WTC 2 are plotted versus the null point number .....

Figure $\mathrm{H}-19$. A drawing of the damage to the steel facade of WTC 2

Figure $\mathrm{H}-20$. The condition of windows is shown for the four faces of WTC 2 around 9:03 a.m. shortly after it was struck by United Airlines Flight 175

Figure H-21. An enhanced photograph of the north face of WTC 2 taken at 9:58 a.m. shows the east side of the north face 
Figure H-22. Images of the east face of WTC 2 taken shortly after the plane struck and shortly before the tower collapse H-38 


\section{LIST OF TABLES}

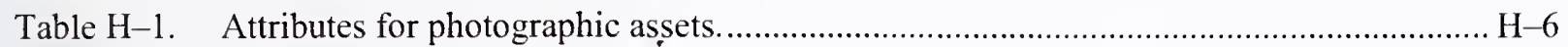

Table H-2. Attributes for video assets. ....................................................................................... H-9

Table H-3. Times for major events of September 11, 2001 ........................................................ H-15 
This page intentionally left blank. 


\section{Appendix $\mathrm{H}$ \\ INTERIM REPORT ON EVOLUTION OF WTC FIRES, SMOKE, AND DAMAGE BASED ON IMAGE ANALYSIS}

\section{H.1 COLLECTION AND ANALYSIS OF VISUAL MATERIAL}

Photographic and video images of damage and fires in the World Trade Center (WTC) towers and WTC 7 are critical for guiding the investigation led by the National Institute of Standards and Technology (NIST). The conditions of the towers immediately following the plane strikes, the rates of fire development and spread through the buildings, and indications as to the floors on which the structural collapses may have begun and their causes are examples of issues that are being addressed using imagery. Observations discussed below demonstrate the importance of such visual evidence.

This appendix is designed to provide an update on NIST efforts to collect and analyze visual material available for the WTC disaster. This effort is part of Project 5, Reconstruction of Thermal and Tenability Environment, and this is the focus of the material presented. It is important to recognize that the effort is coordinated with the other projects that form the NIST-led WTC Investigation, and the visual material is being used as the basis for additional analysis in these projects.

The amount of visual material recorded on September 11,2001, was extraordinary. The terrorist attacks occurred in an area that is the national home base of several news organizations, has several major newspapers, and is the center of the fashion industry. As a result, there were likely hundreds of professional photographers and videographers equipped with excellent equipment and the knowledge to use it in the immediate area. New York City is also a major tourist destination, and visitors often carry cameras to record their visits.

The WTC towers (WTC 1 and WTC 2) were immense, and they dominated the New York City skyline. When WTC 1 was struck by American Airlines Flight 11 around 8:46 a.m., the approach of the plane was captured by at least two videographers who were coincidentally filming nearby. Other photographers and videographers in the vicinity began recording within a few seconds of the impact. As fires grew in the tower, smoke pouring from the building formed a plume that could be seen for miles in all directions in the clear air of September 11, 2001. People in Manhattan, Brooklyn, Queens, and New Jersey began to turn their cameras toward the WTC complex. The major news organizations began coverage almost immediately and began moving professionals into position to cover the event. Numerous other videographers and photographers, both professional and amateur, started moving toward the WTC in order to create their own visual records.

At the time United Airlines Flight 175 struck WTC 2, around 9:03 a.m., the approach and collision of the aircraft were recorded by numerous cameras from a variety of directions. Many people continued to record images until WTC 2 collapsed, around 9:59 a.m. Following this collapse, the amount of visual material decreased markedly as people rushed to escape the area and the huge dust clouds generated by the collapse obscured the site. This situation was only exacerbated by the collapse of WTC 1 , around 
10:28 a.m. The visual record between the period following the collapse of WTC 1 and the collapse of WTC 7, around 5:21 p.m., is much less complete, but there is still a substantial amount of material.

Even as the disaster unfolded, it was clear that a large amount of visual material was being recorded that was being used to inform the public, demonstrate the immensity of the disaster, and to chronicle the associated human suffering. It is now clear that the imagery of September 11,2001, is the most extensive ever recorded of such a single tragic event. The resulting visual record offers an unparalleled opportunity to contribute to the technical understanding of the tragedy of September 11. Even though it was clear that an extensive visual record of the events of September 11 existed, approaches for obtaining access to photographs and videos and cataloging the material had to be developed. These critical aspects of the task have required a great deal of time and effort.

\section{H.1.1 Sources}

Potential sources of visual material have been identified in a number of ways. Recordings of newscasts from September 11, 2001, and afterwards, documentaries and other remembrances, provided information directly, but also pointed toward other potential sources of material. The major photo clearinghouses, such as AP, Reuters, and Corbis, have World Wide Web sites that were reviewed for material related to September 11. Several members of the media suggested sources. Several collections of visual material have been assembled for charitable or historical purposes. Collections from the Here is New York City exhibition and the September 11 Digital Archive were reviewed. Many photographs and videos began appearing on the Web as early as September 11. These could often be identified by Web searches, and in many cases contact information was provided. Public appeals for visual material were made during Investigation news conferences and updates. News accounts of these events led many to contact NIST using the toll-free number or the Investigation Web site. Frequently, a new source would provide information about other potential sources.

NIST hired a visual media consultant, Mr. Valentine Junker, to act as its representative in the New York City area. In addition to interacting with a number of individuals, his efforts were particularly valuable in interfacing with the major television networks and local New York City stations as well as the major photographic news services.

\section{H.1.2 Procedures}

The identification of sources was only the first step in the collection process. It was then necessary to contact the source, request the material, and make arrangements for its transfer. Special considerations such as copyright and privacy issues often needed to be addressed. Once an agreement was reached, arrangements were made to review and transfer copies of the material to NIST.

In the collection process, emphasis has been placed on obtaining material in a form that is as close as possible to the original in order to maintain as much spatial and timing information as possible. In the case of digital photographs and videos this implies a direct digital copy. For film or slide photographs, it would be a high-resolution digitized version of the original media, and for analog video, a direct copy from the original source. While it was not always possible to maintain this standard, the majority of material ultimately collected was handled in this manner. 


\section{H.1.3 Contents}

Significant progress has been made in collecting visual material related to September 11, 2001. Thus far, in excess of 150 hours of video have been assembled. At the time of preparation of this update, video footage has been provided by NBC, CBS, ABC, CNN and local New York City stations WABC, WCBS, WNBC, WPIX, WNYW and New York Citý One. In many cases, the videos provided not only include material broadcast (known as air checks), but also material that was recorded but not broadcast (known as outtakes). Additionally, videotapes recorded by more than 20 individuals have been received.

Photographs have been provided by a number of sources dominated by commercial photo services, the New York City Police Department (NYPD), the New York City Fire Department (FDNY), and individuals. Well in excess of 6,000 photographs, representing more than 185 photographers, have been received. Professional news organizations that have provided material include AP, Corbis, Reuters, the New York City Times, the Daily News, and the Star Ledger. As for the videos, many of these organizations have provided access to unpublished photographs. The majority of photographs have come from individual photographers, both professional and amateur.

It is difficult to estimate the actual amount of relevant visual material recorded on September 11, 2001, and thus to estimate how complete the collection efforts have been. There is certainly material that has not been identified and collected. However, NIST believes that the extraordinarily large collection of video material that it possesses is sufficient for the Investigation.

\section{H.2 DATABASING AND CATALOGING}

It would be impossible to effectively use the vast amount of visual material collected for the Investigation without some means of organizing and cataloging the material.

\section{H.2.1 Digital Storage}

Very early in the task, the decision was made to save all material in digital format on large digital data storage devices. This approach has several advantages. Because the material is in digital form, it can be assessed quickly. It is not necessary to search for a particular photographic collection or videotape, and no special equipment is required to display it. Because most material is received in other forms, the digital storage is in effect a backup system for the original. Additional redundancy is provided by backing up the entire digital storage system at regular intervals. Because videos are saved digitally, they can be analyzed using a variety of commercially available editing software.

Various storage solutions were considered. An approach was finally adopted in which a central server along with two 325 gigabyte and one 160 gigabyte external hard drives were connected with eight personal computers equipped with 70 gigabyte hard drives. The personal computers not only provide additional disk storage, but also serve as workstations for data entry and analysis. All of the systems are connected by high-speed ethernet to form a single network configured such that the entire system becomes, in effect, a single mass storage device. The total amount of storage available is roughly 1.4 terabytes. 
Due to security concerns related to the sensitive nature of some of the visual material and copyright issues, the computer network has been set up with its own dedicated connections and is isolated from the internet backbone of NIST. Policies have been adopted that require all viewing and analysis of the material to be done in secured rooms using secured networks.

\section{H.2.2 Digitizing Techniques}

When new visual material is received at NIST, it is stored digitally on the dedicated system. If the material is already in digital form this simply means copying and saving it on the system. Analog material must be first digitized in some manner. For instance, a photograph might be scanned and digitized, or a video might be converted to a digital video format (typically mini-DV) and then copied to a hard disk.

Each arriving video is logged into VideoList, a Microsoft Access database written specifically for this application. There it is assigned a unique identification number. Pertinent information concerning the tape is recorded, including its duration, the network and broadcast date if applicable, its physical format (e.g., VHS, Hi-8, or mini-DV), where the tape is stored, whether the tape is an original or a copy, its source, whether it has been digitized, whether it contains embedded timecode, and general notes on its content. Figure $\mathrm{H}-1$ shows an example of the entry sheet for the VideoList database. Videos to be stored digitally are copied onto mini-DV media, and each copy is also logged into the database. VideoList also contains a calculator for assisting in the calculation of clip timing that is described in H.3.1. Selected video material is then transferred to hard disk for storage. Video material is often found to have natural breaks, such as when the camera is turned off and on (e.g., by an individual videographer) or when multiple cameras are used (e.g., during a newscast). It is advantageous to treat each of these breaks as the end of an individual video. This is accomplished by a process known as "clipping." By using Adobe Premiere software and a personal computer to control the video player, it is possible to identify and note such breaks in a "clip file." The clip file can also contain notes related to the material. Once a clip file has been generated for an entire tape, the software goes through and automatically generates multiple data files containing the video material. The material is stored in "avi" format, which maintains all of the digital information. The maximum video file size that can be handled by this system is 1 gigabyte. This corresponds to slightly more than 4 1/2 min of avi video. Longer continuous video segments are broken into lengths having roughly this period. Breaking longer videos up in this manner also makes them easier to search and catalog.

\section{H.2.3 Searchable Database}

As noted earlier, a vast amount of visual material has been collected and saved digitally as part of the investigation. Without some organization, it would be impossible to use this material effectively. A commercial database program written specially for organizing visual material, Cumulus, was chosen for this purpose. This software is designed to collect individual "assets" in specified catalogs and to allow the assets to be characterized with a variety of attributes. It is possible to generate specific attributes and include these in specially designed forms for data entry. Once a catalog has been assembled, it is possible to search for assets having a specific attribute or combinations of attributes. Quite sophisticated searches can be created. It is also possible to order assets based on a particular attribute. As an example, when dates and times are assigned, the assets can be ordered in chronological order. 


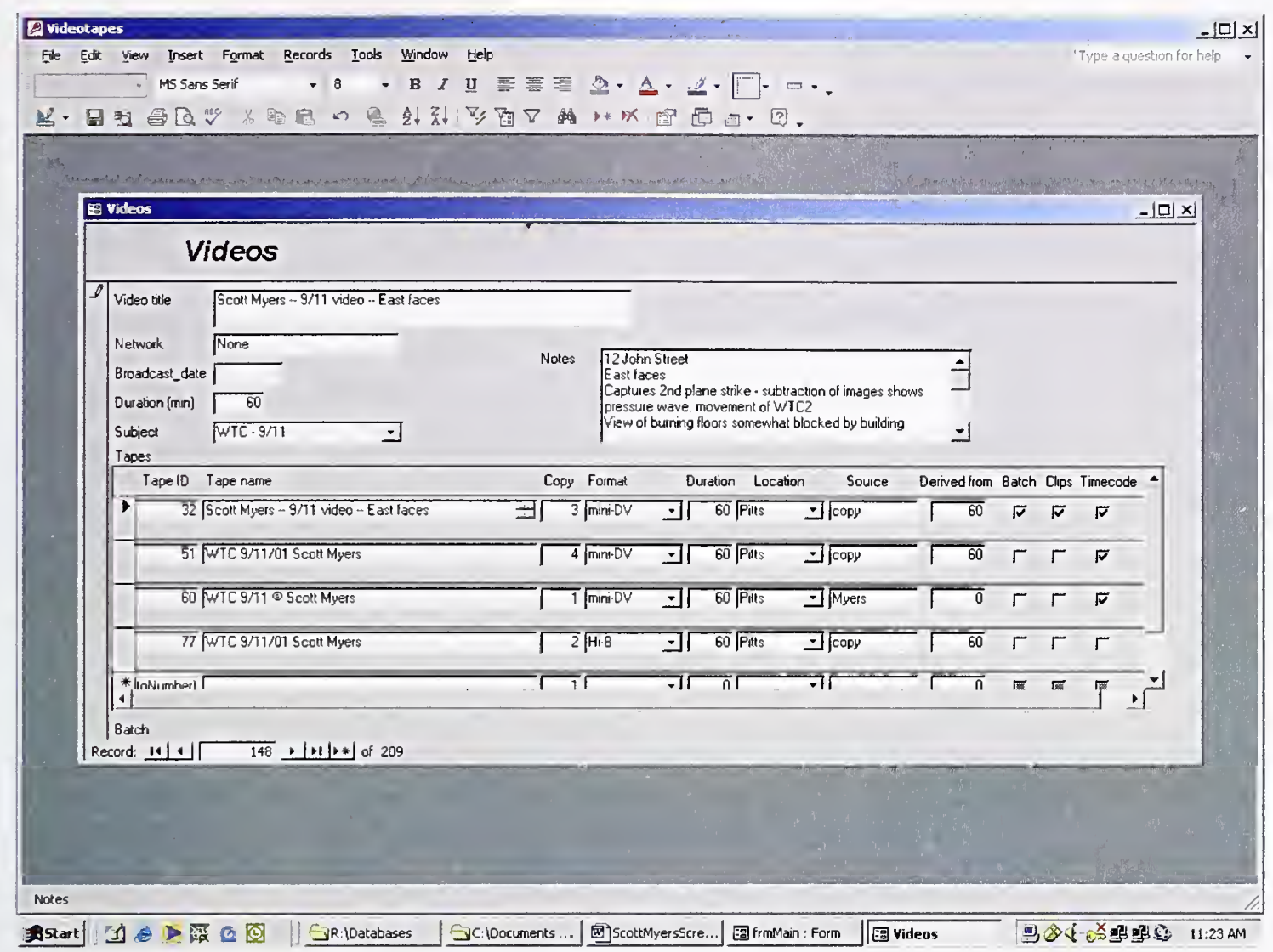

Figure H-1. An example of the VideoList data entry sheet for video assets.

Two separate catalogs, one for photographs and one for video clips, have been created for visual materials collected as part of the Investigation. Each catalog has a similar set of attributes that is used to characterize the assets that are included. These attributes were chosen based on the needs of this task dealing with fire conditions within WTC 1,2 , and 7 and by consultation with members of other project teams. Tables $\mathrm{H}-1$ and Table $\mathrm{H}-2$ list the attributes used for photographic and video catalogs, respectively. A description of each attribute is provided along with details on how information concerning the attribute is input into the worksheet. Figure $\mathrm{H}-2$ shows an example of the first screen for the photographic data entry form.

Cumulus allows thumbnails of entire catalogs or selected subsets to be displayed. This makes it possible to review large numbers of photographs and video clips quickly and to decide which are most likely to be useful for a particular purpose. A variety of asset characteristics can also be shown simultaneously. Typically, the asset name and the time the asset was recorded are displayed. Figure $\mathrm{H}-3$ shows an example of thumbnails taken from the video database. 
Table H-1. Attributes for photographic assets.

\begin{tabular}{|c|c|c|}
\hline Attribute & Definition & Entry Choice \\
\hline Asset Reference & Location of photograph in file system & Set by Cumulus \\
\hline Categories & $\begin{array}{l}\text { List of categories under which the photograph } \\
\text { is listed, typically the photographer's name or } \\
\text { source }\end{array}$ & Set by Cumulus \\
\hline Record Name & File name of photograph & Set by Cumulus \\
\hline Photographer & Photographer's name & Text \\
\hline Received from & $\begin{array}{l}\text { Where photograph was obtained ("Other" may } \\
\text { refer to a third party, for example) }\end{array}$ & $\begin{array}{l}\text { Photographer } \\
\text { WWW } \\
\text { Other }\end{array}$ \\
\hline Original Source & How photograph was added to the collection & $\begin{array}{l}\text { Digital Copy of Original } \\
\text { Digital Copy from Program } \\
\text { Digitized Slide or Negative } \\
\text { Digitized Photograph } \\
\text { Uploaded from Web }\end{array}$ \\
\hline Use Limited & $\begin{array}{l}\text { Photographer has requested that use of the } \\
\text { photograph be limited }\end{array}$ & Checkbox \\
\hline Copyright & A copyright exists & Checkbox \\
\hline Copyright Agreement & Usage agreement with NIST & Text \\
\hline Shot From & Location of photographer & Text \\
\hline Date Recorded & Date and time of shot & Date and time \\
\hline Time Uncertainty (s) & $\begin{array}{l}\text { Number of seconds uncertainty in the time } \\
\text { assigned }\end{array}$ & Integer \\
\hline View Direction & $\begin{array}{l}\text { Location of photographer with respect to the } \\
\text { WTC }\end{array}$ & $\begin{array}{l}\text { North } \\
\text { Northeast } \\
\text { East } \\
\text { Southeast } \\
\text { South } \\
\text { Southwest } \\
\text { West } \\
\text { Northwest }\end{array}$ \\
\hline $\begin{array}{l}\text { WTC Faces } \\
\text { WTC } 1 \text { North Face } \\
\text { WTC } 1 \text { East Face } \\
\text { WTC } 1 \text { South Face } \\
\text { WTC } 1 \text { West Face } \\
\text { WTC } 2 \text { North Face } \\
\text { WTC } 2 \text { East Face } \\
\text { WTC } 2 \text { South Face } \\
\text { WTC } 2 \text { West Face } \\
\text { WTC } 7 \text { North Face } \\
\text { WTC } 7 \text { East Face } \\
\text { WTC } 7 \text { South Face } \\
\text { WTC } 7 \text { West Face }\end{array}$ & Building face(s) visible in the photograph & Checkbox for each choice \\
\hline
\end{tabular}




\begin{tabular}{|c|c|c|}
\hline Attribute & Definition & Entry Choice \\
\hline $\begin{array}{l}\text { Distance } \\
\text { Near } \\
\text { Medium } \\
\text { Far }\end{array}$ & $\begin{array}{l}\text { Clarity of the photograph } \\
\text { Near = Can make out details in windows } \\
\text { Medium = Can count windows } \\
\text { Far = Unable to count windows }\end{array}$ & Checkbox for each choice \\
\hline $\begin{array}{l}\text { Building } \\
\text { WTC } 1 \\
\text { WTC } 2 \\
\text { WTC } 7 \\
\text { Other Building } \\
\end{array}$ & Building(s) visible in photograph & Checkbox for each choice \\
\hline 1st Plane Strike & Photograph shows the plane strike on WTC 1 & Checkbox \\
\hline 2nd Plane Strike & Photograph shows the plane strike on WTC 2 & Checkbox \\
\hline WTC 1 Collapse & Photograph shows the collapse of WTC 1 & Checkbox \\
\hline WTC 2 Collapse & Photograph shows the collapse of WTC 2 & Checkbox \\
\hline WTC 7 Collapse & Photograph shows the collapse of WTC 7 & Checkbox \\
\hline Street & $\begin{array}{l}\text { Street scene, or a street is visible in the } \\
\text { photograph }\end{array}$ & Checkbox \\
\hline $\begin{array}{l}\text { Debris } \\
\text { Aircraft Debris } \\
\text { Collapse Debris } \\
\text { Debris Inside Building } \\
\text { Street Debris }\end{array}$ & $\begin{array}{l}\text { Debris is visible in the photograph } \\
\text { Type of debris: } \\
\text { Aircraft = Can be identified as plane debris } \\
\text { (e.g., tires, engines) } \\
\text { Collapse = Resulting from collapse } \\
\text { Inside Building = Visible through windows } \\
\text { or openings } \\
\text { Street = On street }\end{array}$ & Checkbox for each choice \\
\hline Fireball & Initial fireball from plane strike is visible & Checkbox \\
\hline Thermal & $\begin{array}{l}\text { The thermal is a tall region of the smoke plume } \\
\text { that results from the lift caused by the hot gases } \\
\text { of the initial fireball }\end{array}$ & Checkbox \\
\hline Plume & $\begin{array}{l}\text { Smoke plume generated by the fires within the } \\
\text { towers and blown downwind. This marker is } \\
\text { checked if the smoke plume in the photograph } \\
\text { extends farther than a single tower width. }\end{array}$ & Checkbox \\
\hline Flames Visible & Flames are visible in the photograph & Checkbox \\
\hline $\begin{array}{l}\text { People } \\
\text { Inside } \\
\text { Falling } \\
\text { Outside }\end{array}$ & $\begin{array}{l}\text { The photograph includes people } \\
\text { Inside = People inside the buildings, at the } \\
\text { windows or climbing down } \\
\text { Outside = People on the street }\end{array}$ & Checkbox for each choice \\
\hline $\begin{array}{l}\text { Falling building } \\
\text { component }\end{array}$ & $\begin{array}{l}\text { The photograph shows a building component } \\
\text { falling (e.g., aluminum cladding) }\end{array}$ & Checkbox \\
\hline Streamers Falling & $\begin{array}{l}\text { The photograph shows a streamer, an object } \\
\text { that emits smoke as it falls and leaves a trail }\end{array}$ & Checkbox \\
\hline Dripping & $\begin{array}{l}\text { Molten material dripping from WTC } 2 \text { is } \\
\text { visible }\end{array}$ & Checkbox \\
\hline Hanging Floor & $\begin{array}{l}\text { A sagging or hanging object suggesting a floor } \\
\text { is visible within the windows }\end{array}$ & Checkbox \\
\hline
\end{tabular}




\begin{tabular}{|c|c|c|}
\hline Attribute & Definition & Entry Choice \\
\hline Building Core & $\begin{array}{l}\text { Photograph shows the core of WTC } 1 \text { or } \\
\text { WTC } 2 \text { - both remained standing briefly } \\
\text { during collapse before falling }\end{array}$ & Checkbox \\
\hline $\begin{array}{l}\text { FDNY } \\
\text { FDNY Apparatus } \\
\text { FDNY Personnel }\end{array}$ & $\begin{array}{l}\text { FDNY personnel or vehicles are visible, } \\
\text { including EMTs, fire trucks, and ambulances }\end{array}$ & Checkbox for each choice \\
\hline $\begin{array}{l}\text { NYPD } \\
\text { NYPD Apparatus } \\
\text { NYPD Personnel }\end{array}$ & $\begin{array}{l}\text { NYPD personnel or vehicles are visible, also } \\
\text { includes FBI and other police officials }\end{array}$ & Checkbox for each choice \\
\hline Impact Aircraft & $\begin{array}{l}\text { Photograph shows aircraft approaching WTC } 1 \\
\text { or WTC } 2 \text { before or during the strike }\end{array}$ & Checkbox \\
\hline Other Aircraft & $\begin{array}{l}\text { Aircraft other than the impact aircraft are } \\
\text { included in the photograph, such as helicopters } \\
\text { or fighter jets }\end{array}$ & Checkbox \\
\hline Good for Analysis & $\begin{array}{l}\text { Mark photograph for possible window-by- } \\
\text { window analysis }\end{array}$ & Checkbox \\
\hline Analyzed & $\begin{array}{l}\text { The photograph has been used for window-by- } \\
\text { window analysis }\end{array}$ & Checkbox \\
\hline Notes & $\begin{array}{l}\text { Notes, including a description of how the } \\
\text { photograph was timed }\end{array}$ & Checkbox \\
\hline
\end{tabular}

Not all collected visual material is incorporated into the two catalogs. Photographs and videos judged not to contain information directly relevant to the Investigation are not included. Even so, the number of photographs and clips included in the catalogs is huge. At the time of writing, the photographic catalog includes 6,759 assets and the video catalog includes 6,911 assets.

\section{H.3 TIMING OF PHOTOGRAPHS AND VIDEO CLIPS}

Since one of the major goals of this task is the development of time lines for fire growth and spread in WTC 1,2, and 7, it is important to assign times of known accuracy to assets included in the two catalogs. This task is complicated by the absence of accurate times for the majority of visual materials collected.

\section{H.3.1 Digital Timestamps}

Modern photographic and video digital cameras often record camera clock times as part of their output. For photographs, this information is usually stored as an integral part of the image in a header known as an Exif file. Similarly, digital video cameras often embed a variety of information, including the camera clock time, as part of what is known as meta data. Software is available for reading these clock times from Exif and other meta data media file formats. While a great help, these times usually still require some adjustment because people do not generally set their camera clocks accurately. In some cases, camera clocks were off by days or even years. Even so, the relative times over the short time period of the events of September 11, 2001, are quite accurate. 
Table H-2. Attributes for video assets.

\begin{tabular}{|c|c|c|}
\hline Attribute & Definition & Entry Choice \\
\hline Asset Reference & Location of video clip in the file system & Set by Cumulus \\
\hline Categories & $\begin{array}{l}\text { List of categories under which the video clip is } \\
\text { listed, typically the photographer's name or } \\
\text { source }\end{array}$ & Set by Cumulus \\
\hline Record Name & File name of video clip & Set by Cumulus \\
\hline Photographer & Photographer's name & Text \\
\hline Content & $\begin{array}{l}\text { Content of video clip } \\
\text { WTC } 9 / 11 \text { Footage = Events before collapse } \\
\text { of WTC } 7 \\
\text { Street Scene (no timing) } \\
\text { Debris field = Ground Zero after WTC } 7 \\
\text { collapse } \\
\text { Construction = Construction of WTC towers } \\
\text { from documentary } \\
\text { Normal Operation = Normal operation of } \\
\text { building, usually from documentary } \\
\text { Animation = Animation of } 9 / 11 \text { events from } \\
\text { documentary } \\
\text { Still(s) = Photographs contained within } \\
\text { documentary } \\
\text { Interview = Clip only shows interview }\end{array}$ & $\begin{array}{l}\text { WTC } 9 / 11 \text { Footage } \\
\text { Street scene (no timing) } \\
\text { Debris field } \\
\text { Construction } \\
\text { Normal operation } \\
\text { Animation } \\
\text { Still(s) } \\
\text { Interview }\end{array}$ \\
\hline Use Limited & $\begin{array}{l}\text { Videographer has requested that use of the } \\
\text { videotape be limited }\end{array}$ & Checkbox \\
\hline Copyright & A copyright exists & Checkbox \\
\hline Copyright Agreement & Usage agreement arrangements with NIST & Text \\
\hline Shot From & Location of videographer & Text \\
\hline Date Recorded & Date and time of beginning of video clip & Date and time \\
\hline End Recording & Date and time of end of video clip & Date and time \\
\hline Duration & Number of minutes:seconds contained in clip & Real number \\
\hline Time Uncertainty (s) & $\begin{array}{l}\text { Number of seconds uncertainty in the time } \\
\text { recorded / end recording }\end{array}$ & Integer \\
\hline View Direction & $\begin{array}{l}\text { Location of videographer with respect to the } \\
\text { WTC }\end{array}$ & $\begin{array}{l}\text { North } \\
\text { Northeast } \\
\text { East } \\
\text { Southeast } \\
\text { South } \\
\text { Southwest } \\
\text { West } \\
\text { Northwest }\end{array}$ \\
\hline
\end{tabular}




\begin{tabular}{|c|c|c|}
\hline Attribute & Definition & Entry Choice \\
\hline $\begin{array}{l}\text { WTC Faces } \\
\text { WTC } 1 \text { North Face } \\
\text { WTC } 1 \text { East Face } \\
\text { WTC } 1 \text { South Face } \\
\text { WTC } 1 \text { West Face } \\
\text { WTC } 2 \text { North Face } \\
\text { WTC } 2 \text { East Face } \\
\text { WTC } 2 \text { South Face } \\
\text { WTC } 2 \text { West Face } \\
\text { WTC } 7 \text { North Face } \\
\text { WTC } 7 \text { East Face } \\
\text { WTC } 7 \text { South Face } \\
\text { WTC } 7 \text { West Face }\end{array}$ & Building face(s) visible in the video clip & Checkbox for each choice \\
\hline $\begin{array}{l}\text { Distance } \\
\text { Near } \\
\text { Medium } \\
\text { Far }\end{array}$ & $\begin{array}{l}\text { Clarity of the video clip } \\
\text { Near = Can make out details in windows } \\
\text { Medium }=\text { Can count windows } \\
\text { Far = Unable to count windows }\end{array}$ & Checkbox for each choice \\
\hline $\begin{array}{l}\text { Building } \\
\text { WTC } 1 \\
\text { WTC } 2 \\
\text { WTC } 7 \\
\text { Other Building } \\
\end{array}$ & Building(s) visible in video clip & Checkbox for each choice \\
\hline 1st Plane Strike & Clip shows the plane strike on WTC 1 & Checkbox \\
\hline 2nd Plane Strike & Clip shows the plane strike on WTC 2 & Checkbox \\
\hline WTC 1 Collapse & Clip shows the collapse of WTC 1 & Checkbox \\
\hline WTC 2 Collapse & Clip shows the collapse of WTC 2 & Checkbox \\
\hline WTC 7 Collapse & Clip shows the collapse of WTC 7 & Checkbox \\
\hline Street & $\begin{array}{l}\text { Street scene, or a street is visible in the video } \\
\text { clip }\end{array}$ & Checkbox \\
\hline $\begin{array}{l}\text { Debris } \\
\text { Aircraft Debris } \\
\text { Collapse Debris } \\
\text { Debris Inside Building } \\
\text { Street Debris }\end{array}$ & $\begin{array}{l}\text { Debris is visible in the video clip } \\
\text { Type of debris: } \\
\text { Aircraft = Can be identified as plane debris } \\
\text { (e.g., tires, engines) } \\
\text { Collapse = Resulting from collapse } \\
\text { Inside Building = Visible through windows } \\
\text { Street = On street }\end{array}$ & Checkbox for each choice \\
\hline Fireball & Initial fireball from plane strike is visible & Checkbox \\
\hline Thermal & $\begin{array}{l}\text { The thermal is a tall region of the smoke plume } \\
\text { that results from the lift caused by the hot gases } \\
\text { of the initial fireball }\end{array}$ & Checkbox \\
\hline Plume & $\begin{array}{l}\text { Smoke plume generated by the fires within the } \\
\text { towers and blown downwind. This marker is } \\
\text { checked if the smoke plume in the video clip } \\
\text { extends farther than a single tower width. }\end{array}$ & Checkbox \\
\hline Flames Visible & Flames are visible in the video clip & Checkbox \\
\hline
\end{tabular}




\begin{tabular}{|c|c|c|}
\hline Attribute & Definition & Entry Choice \\
\hline $\begin{array}{l}\text { People } \\
\text { Inside } \\
\text { Falling } \\
\text { Outside }\end{array}$ & $\begin{array}{l}\text { The video clip includes people } \\
\text { Inside = People inside the buildings, at the } \\
\text { windows, or climbing down } \\
\text { Outside = People on the street }\end{array}$ & Checkbox for each choice \\
\hline Falling building component & $\begin{array}{l}\text { The video clip shows a building component } \\
\text { falling (e.g., aluminum cladding) }\end{array}$ & Checkbox \\
\hline Streamers Falling & $\begin{array}{l}\text { The video clip shows a streamer, an object that } \\
\text { emits smoke as it falls and leaves a trail }\end{array}$ & Checkbox \\
\hline Dripping & $\begin{array}{l}\text { Molten material dripping from WTC } 2 \text { is } \\
\text { visible }\end{array}$ & Checkbox \\
\hline Hanging Floor & $\begin{array}{l}\text { A sagging object suggesting a floor is visible } \\
\text { within the windows }\end{array}$ & Checkbox \\
\hline Building Core & $\begin{array}{l}\text { Video clip shows the core of WTC } 1 \text { or } \\
\text { WTC } 2 \text { - both remained standing briefly during } \\
\text { collapse before falling }\end{array}$ & Checkbox \\
\hline $\begin{array}{l}\text { FDNY } \\
\text { FDNY Apparatus } \\
\text { FDNY Personnel } \\
\end{array}$ & $\begin{array}{l}\text { FDNY personnel or vehicles are visible, } \\
\text { including EMTs, fire trucks, and ambulances }\end{array}$ & Checkbox for each choice \\
\hline $\begin{array}{l}\text { NYPD } \\
\text { NYPD Apparatus } \\
\text { NYPD Personnel }\end{array}$ & $\begin{array}{l}\text { NYPD personnel or vehicles are visible, also } \\
\text { includes FBI and other police officials }\end{array}$ & Checkbox for each choice \\
\hline $\begin{array}{l}\text { Aircraft } \\
\text { Impact Aircraft } \\
\text { Other Aircraft }\end{array}$ & $\begin{array}{l}\text { Aircraft are visible in the video clip } \\
\text { Impact: Shows aircraft approaching WTC } 1 \\
\text { or WTC } 2 \text { before or during the strike } \\
\text { Other: Helicopters or fighter jets }\end{array}$ & Checkbox for each choice \\
\hline $\begin{array}{l}\text { Major Change } \\
\text { Major Fire Change } \\
\text { Major Smoke Change } \\
\text { Windows Opened }\end{array}$ & $\begin{array}{l}\text { One of the following events takes place in the } \\
\text { video clip: } \\
\text { Major Fire Change: Fire flares up, dies } \\
\text { down, or spreads to a new region } \\
\text { Major Smoke Change: Smoke bursts, dies } \\
\text { down, or spreads to a new region } \\
\text { Windows Opened: Window breaks open, } \\
\text { either due to fire or to people }\end{array}$ & Checkbox for each choice \\
\hline Good for Analysis & $\begin{array}{l}\text { Mark video clip for possible window-by- } \\
\text { window analysis }\end{array}$ & Checkbox \\
\hline Analyzed & $\begin{array}{l}\text { The video clip has been used for window-by- } \\
\text { window analysis }\end{array}$ & Checkbox \\
\hline Notes & $\begin{array}{l}\text { Notes, including a description of how the video } \\
\text { clip was timed }\end{array}$ & Text \\
\hline
\end{tabular}




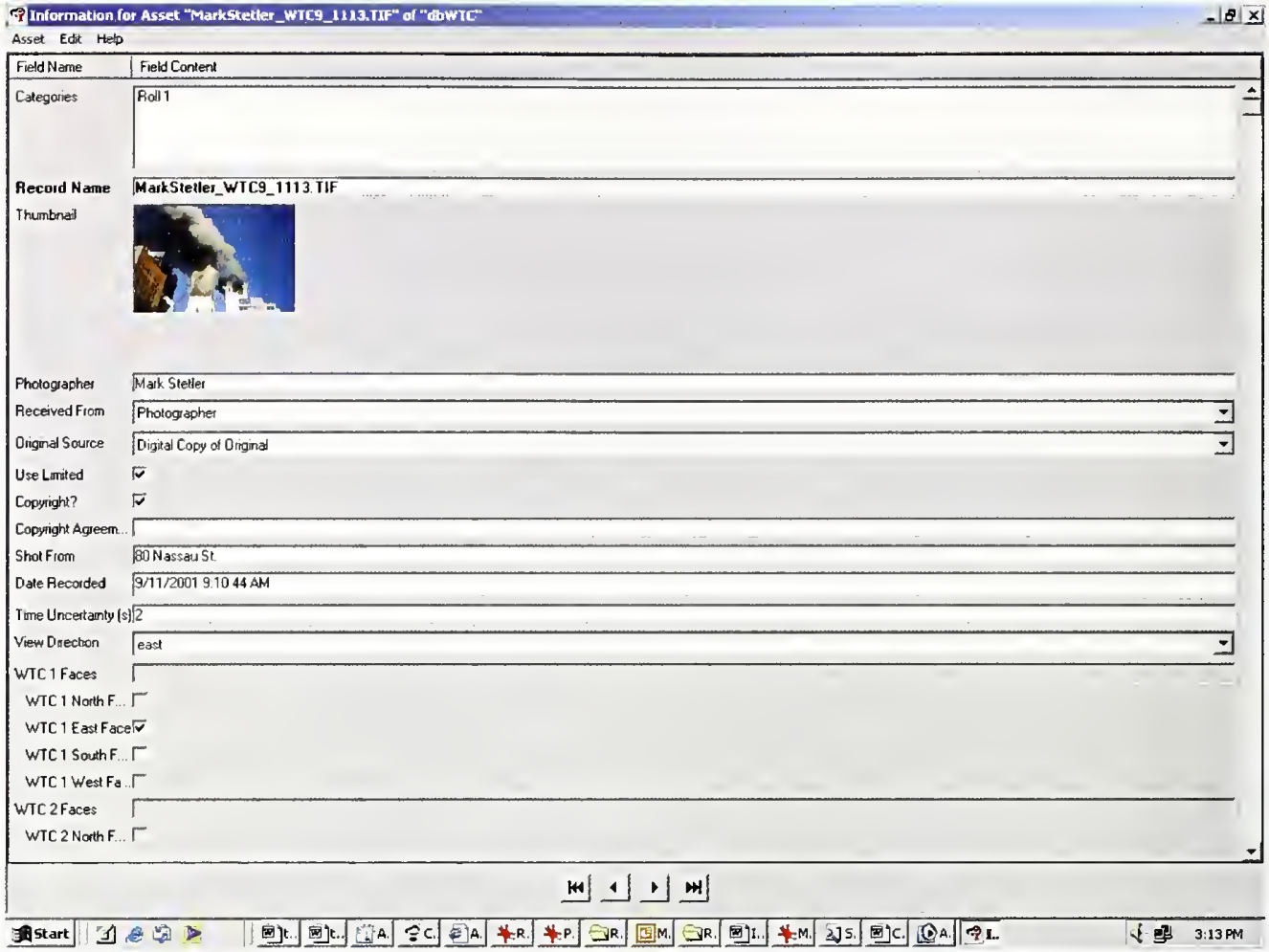

Figure $\mathrm{H}-2$. An example of the first page of the Cumulus data entry sheet for photographic assets. Thumbnail (c) 2001 Mark Stetler.

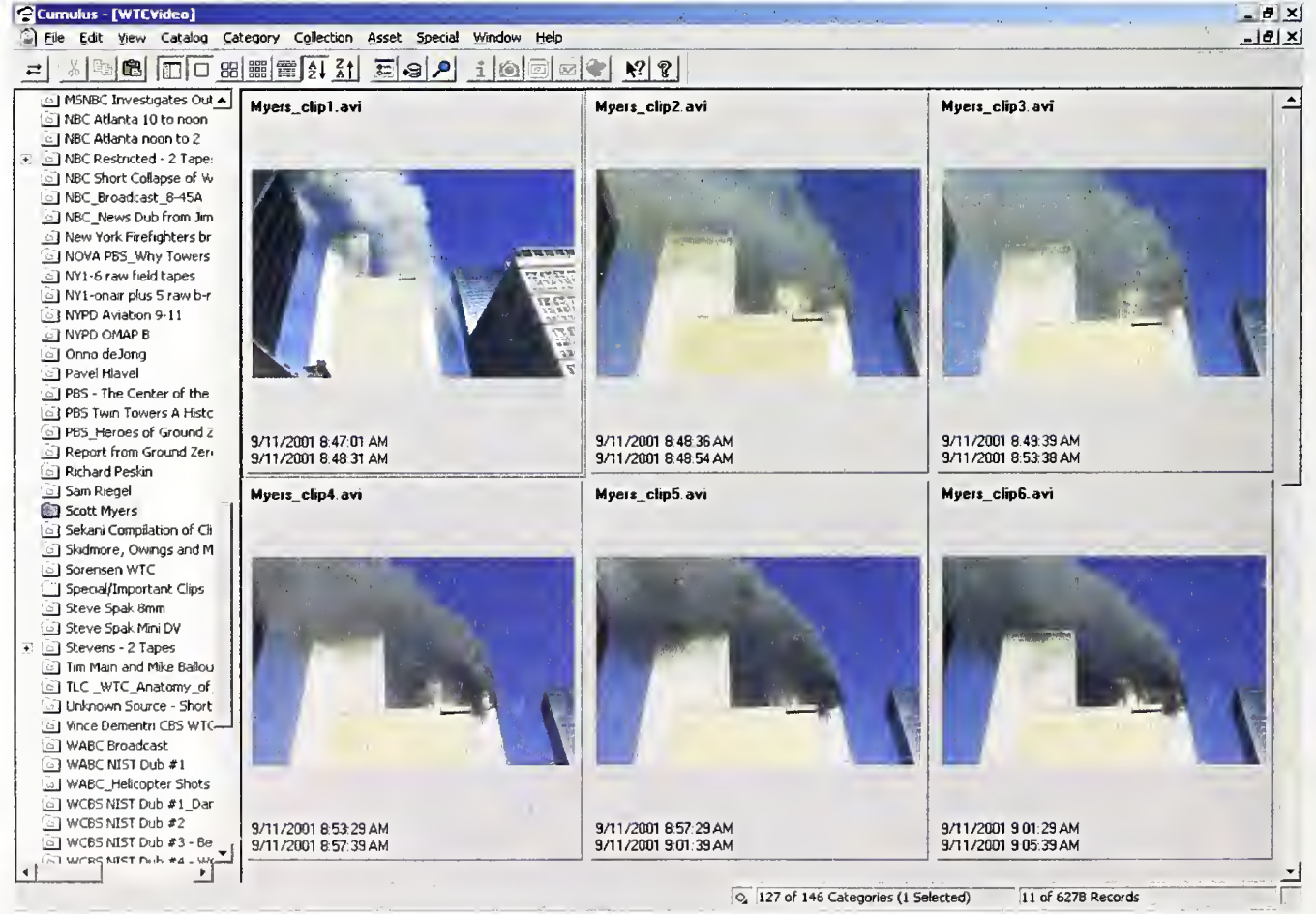

Figure H-3. An example of a Cumulus asset screen display for the video database. Thumbnails are shown along with the time and dates when the recording started and ended. Thumbnails ( 2001 Scott Meyers. 
Occasionally analog photo and video cameras imprint a time stamp on their outputs that can provide relative times similar to Exif or meta data, but generally there is no time information available, and such material must be timed in some other way. Some of the approaches used are described later in this section.

\section{Photograph Tools}

In order to make the best use of the information embedded in digital photographs, software was required to retrieve the Exif file information and software to adjust the recorded clock times. The commercial software package CatDV is able to retrieve meta data embedded in a variety of media formats, including digital photographs and mini-DVs. The Access database PhotoTiming was written for the purpose of determining the actual times for a set of photographs given the Exif time for each and an accurate time reference. For a set of photographs sharing a common clock from the same digital camera, an accurate time for a single photograph is sufficient to set the times for the entire set. Figure $\mathrm{H}-4$ shows a PhotoTiming data sheet for a selected photographer. A file generated by CatDV containing the Exif data for each photograph, if available, is read into PhotoTiming. The equivalent Exif and actual times are entered into the appropriate fields at the upper right of the data sheet. Selection of the Calculate Photo Times button fills the Actual Time column with the appropriate value for each Exif time. In this example, the Exif time was found to be off by $62 \mathrm{~s}$.

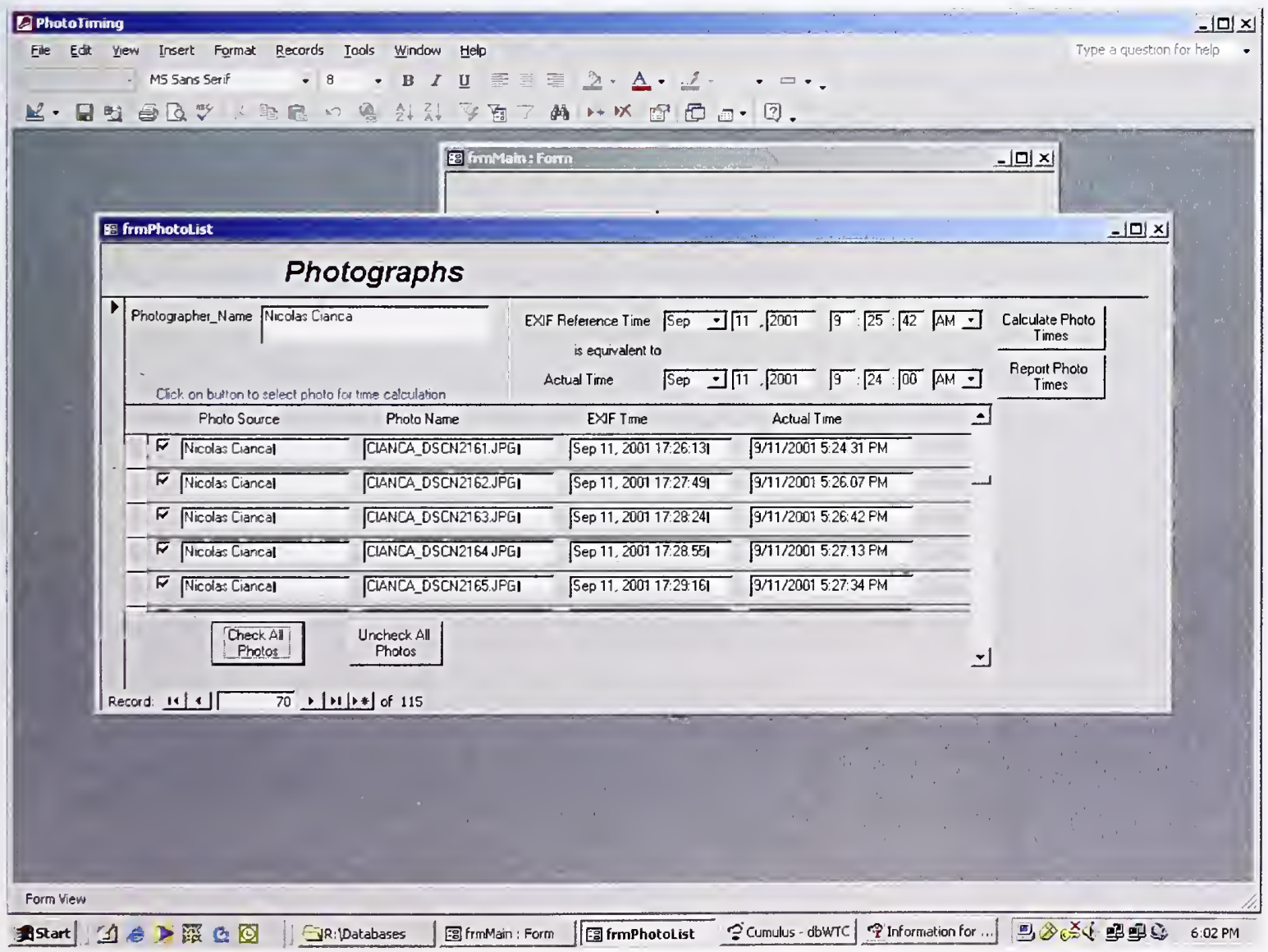

Figure H-4. An example of the PhotoTiming sheet for calculating times for photographs containing Exif meta data. 


\section{Video Tools}

In addition to containing the video database described in Section H.2.3, VideoList also assists with timing the clips from a videotape. This function is similar to that in the PhotoTiming tool. For a broadcast video that was filmed in real time, the timing of every clip in the video, except for replays, can be set from knowing the time at a single point. An example is shown in Figure $\mathrm{H}-5$. A clip file generated in Adobe Premiere for a specified video is read into VideoList. The mini-DV time of an event in the video whose timing is known, such as the moment of the second plane strike, is identified. Both times are entered into the fields at the upper right of the data sheet. Clips to be timed (excluding replays) are identified by a check mark, and the requested calculation results in the actual times in and out for each clip as shown in Figure $\mathrm{H}-5$. This tool is also useful in calculating timings for continuous video segments broken into multiple clips.

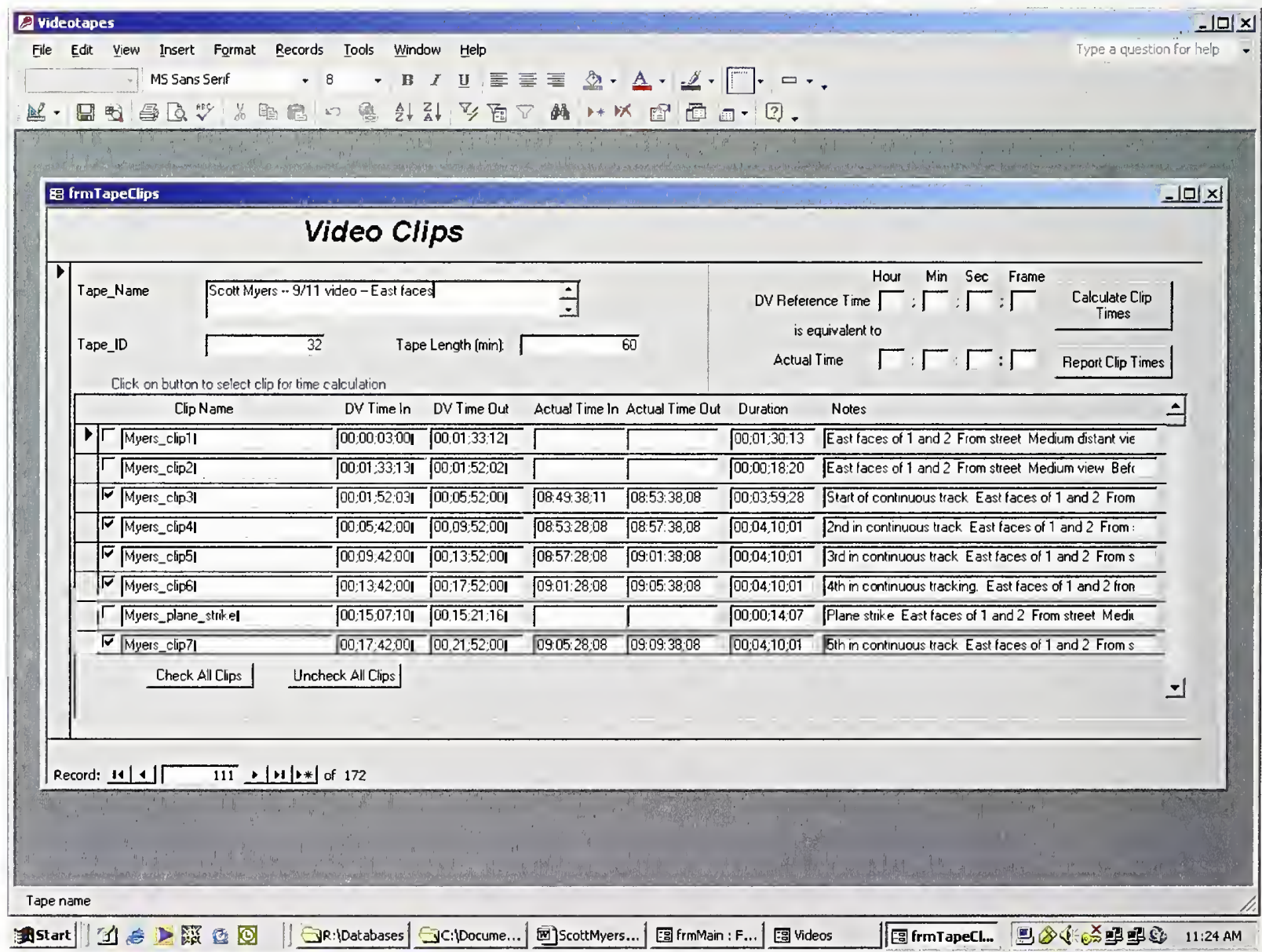

Figure H-5. An example of the VideoList sheet for calculating clip times for video assets.

For each mini-DV video that contains meta data, CatDV is used to extract the clock times for the In and Out point for each clip. These values enable the timing of every clip in the video from a single reference time. 


\section{H.3.2 Reference Time}

Faced with the timing considerations above, a timing scheme was developed in which all of the times in the databases are placed on a relative time scale tied to a single well-defined event. Due to the large number of different views available, the moment the second plane struck WTC 2 was chosen to be this time. This event was defined to have occurréd at 9:02:54 a.m. based on times for major events included in the earlier Federal Emergency Management Agency (FEMA) report (McAllister 2002) describing the events of September 11, 2001.

\section{H.3.3 Timing Techniques}

Once the reference time was chosen, it was possible to place times on videos that showed the second plane strike. By matching other photographs and videos to these initially assigned videos, the assignments were extended to visual materials that did not include the primary event. By such a bootstrap process, it was possible to place photographs and videos extending over the entire period of the event on a single time line. Sets of photographs containing Exif times and video clips that either contained meta data or were continuous over relatively long periods were particularly useful for this purpose because a single time assignment would allow the entire series to be timed. Sets of photographs recorded on film or analog videos that were frequently turned on and off caused the most difficulty in timing, and individual matches were required for each photo or video clip.

Matching visual images and assigning times has turned out to be a demanding task requiring unique approaches. A variety of characteristics have been employed to match times in different photographs and videos. These include distinct shadows cast on the buildings by the smoke plumes, the appearance and locations of smoke and fire plumes, the occurrence of well-defined events such as a falling object or the sudden appearance of smoke, and a variety of other unlikely clues such as a clock being recorded in an image.

To assist in the timing process, relative times for the five major events of September 11, 2001: first plane strike, second plane strike, collapse of WTC 2, collapse of WTC 1, and collapse of WTC 7 have been determined with 1 second accuracy. These times are summarized in Table H-3. Note that the building collapse times are defined to be when the entire building is first observed to start to collapse. In the case of WTC 7, a penthouse on the roof sank into the building before the main collapse started.

Table H-3. Times for major events of September 11, 2001.

\begin{tabular}{|l|c|c|c|}
\hline \multicolumn{1}{|c|}{ Event } & $\begin{array}{c}\text { Relative Time from } \\
\text { Visual Analysis }\end{array}$ & $\begin{array}{c}\text { Adjusted Time from } \\
\text { Television Broadcasts }\end{array}$ & $\begin{array}{c}\text { Time Reported in the } \\
\text { FEMA Study }\end{array}$ \\
\hline First plane strike & $8: 46: 25$ a.m. & $8: 46: 30$ a.m. & $8: 46: 26$ a.m. \\
\hline Second plane strike & $9: 02: 54$ a.m. & $9: 02: 59$ a.m. & $9: 02: 54$ a.m. \\
\hline Collapse of WTC 2 & $9: 58: 54$ a.m. & $9: 58: 59$ a.m. & $9: 59: 04$ a.m. \\
\hline Collapse of WTC 1 & $10: 28: 20$ a.m. & $10: 28: 25$ a.m. & $10: 28: 31$ a.m. \\
\hline Collapse of WTC 7 & $5: 20: 47$ p.m. & $5: 20: 52$ p.m. & $5: 20: 33$ p.m. \\
\hline
\end{tabular}

It is not only important to assign relative times for photographs and videos, but also to estimate how accurately they are known. For this reason, timing uncertainties are estimated for each determination and are included in the databases. 
The bootstrap timing process was initially quite difficult. However, team members' timing skills improved with practice at the same time as more visual material became available and the number of timed assets increased. At the present time, 3,032 of the 6,759 catalogued photographs and 2,673 of the 6,911 video clips in the databases are timed with assigned relative accuracies of 3 seconds or better.

\section{H.3.4 Absolute Time Accuracy}

Many of the news broadcasts on September 11,2001, included small clocks, known in the industry as "bugs," imprinted on the screen. As such broadcasts were timed, it became apparent that there were small differences between times for the second plane strike based on these bugs and the time used as the basis for the database. Checks with several broadcasters indicated that the bugs should be quite close to the actual time because their clocks are regularly updated from highly accurate sources such as geopositioning satellites or the precise atomic-clock-based timing signals provided by NIST as a public service. Careful checks showed small time differences between different video recordings, but these were generally less than 1 second. These small discrepancies are likely due to variations in transmission times resulting from the different pathways that the video signals take to the sites where they are recorded. Based on four such video recordings, the time of the second plane impact is estimated as 9:02:59 a.m., or 5 seconds later than the time assumed in developing the database. The estimated uncertainty is 1 second. Table H-3 compares times for the major events taken from the database, adjusted to television time, and reported in the FEMA report (McAllister 2002). Possible explanations for the observed differences are still under investigation. Because times based on the television broadcasts appear to be accurate (i.e., those in column 3 of Table $\mathrm{H}-3$ ), 5 seconds will be added to times included in the databases when precise times are reported for the Investigation.

\section{H.4 ANALYSIS OF VISUAL IMAGES}

Once the two visual databases became available, it was possible to use the images to begin characterizing the events of September 11, 2001. Some of the images are quite close up and can be used to learn specific details concerning the towers. As an example, Fig. $\mathrm{H}-6$ shows an image of the east face of WTC 2 recorded at 9:26:20 a.m., and Fig. $\mathrm{H}-7$ shows an enlarged portion of the same photograph. The photograph has been enhanced using Adobe Photoshop, and lettering has been added to indicate the floors and the numbering system used to identify specific windows in the tower. The amount of detail available is evident. For instance, large piles of debris are present on the north side of the tower on floors 80 and 81 , and locations with fires visible or with windows missing are easily identified.

\section{H.4.1 Window Numbering}

The system used to describe window locations in the two towers and WTC 7 requires some elaboration. It is based on the outer-wall column numbering system used in plans for the buildings. First, consider the towers. In these structures individual windows were placed between two exterior columns. In order to refer to a particular window the designation for the column to the right as viewed from the outside is assigned to that window. These columns are numbered from 1 to 59 from right to left across a tower face, and windows are numbered from 1 to 58 . Faces for the towers are also assigned numbers as follows; WTC 1-north: 1, east: 2 , south: 3, and west: 4, and WTC 2-west: 1 , north: 2, east: 3 , and south: 4 . By combining the floor number, the face number and a column number, a specific window on one of the 


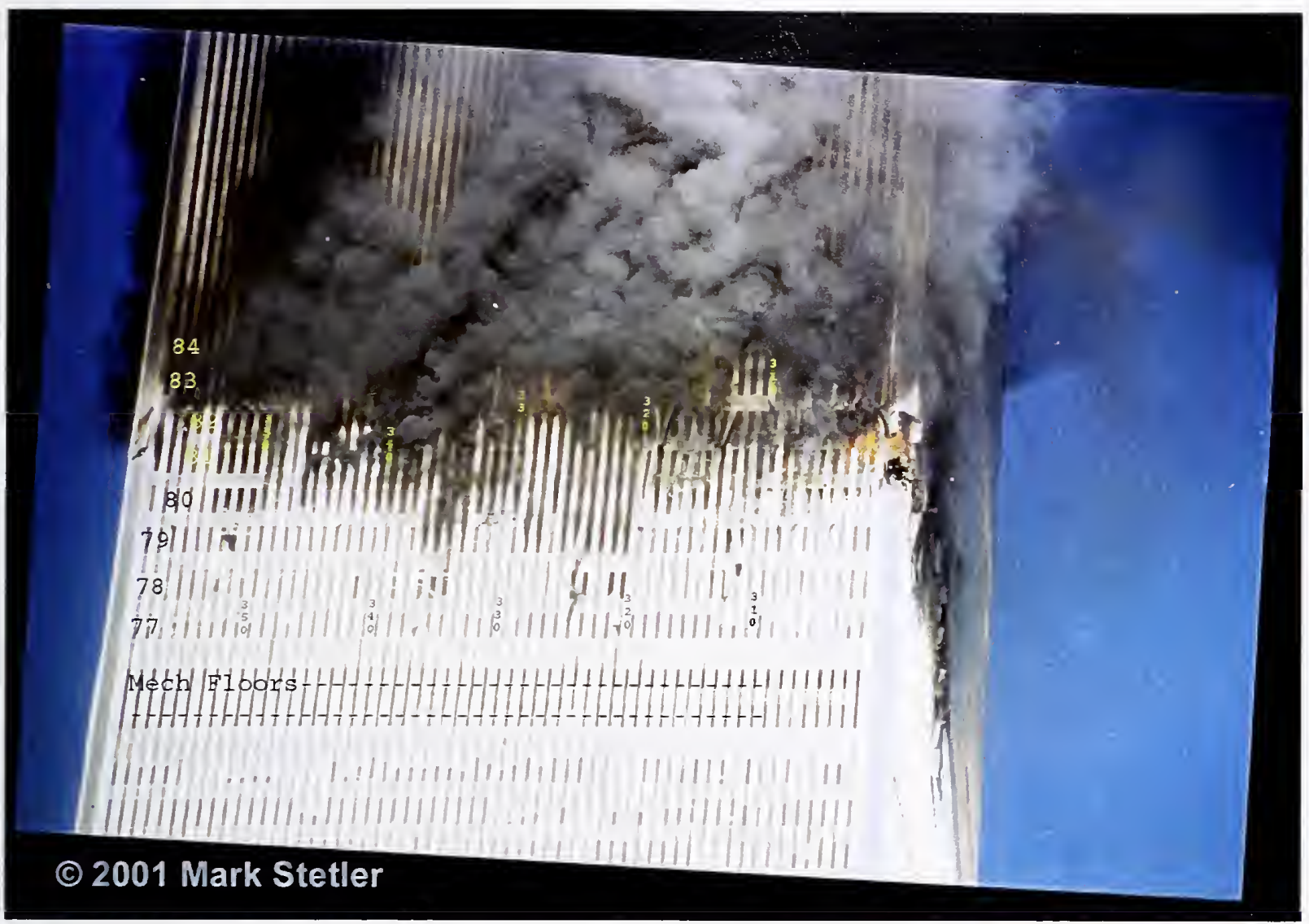

Figure H-6. Photograph taken at 9:26:20 a.m. on September 11, 2001, showing the east face of WTC 2. It has been enhanced, and lettering indicating floors and columns has been added.

towers can be identified. As an example, for WTC 1, the number 94-214 refers to the fourteenth window from the right on the east face of floor 94.

The window numbering system is somewhat different for WTC 7. It is also based on the outside column numbers, but in this building the numbering of columns was continuous around the structure and ranged from 1 to 57. Column 1 was located at the northwest corner of the building, and the numbering proceeded counter clockwise around the building faces with columns 15, 28, and 42 located at the southwest, southeast, and northeast corners, respectively. Note that the total number of perimeter columns is actually 58. An extra column, numbered 14A, was included on the west face between columns 14 and 15. Unlike the towers, the number of windows to the right of a given column varied from one to five depending on location. In some cases, the windows are located in front of the column. Individual windows to the right of a column are assigned letters increasing from left to right as seen from the outside. As an example, 12-45c refers to a window on the north face of WTC 7 that is the third window to the right of column 45 on floor 12 . 


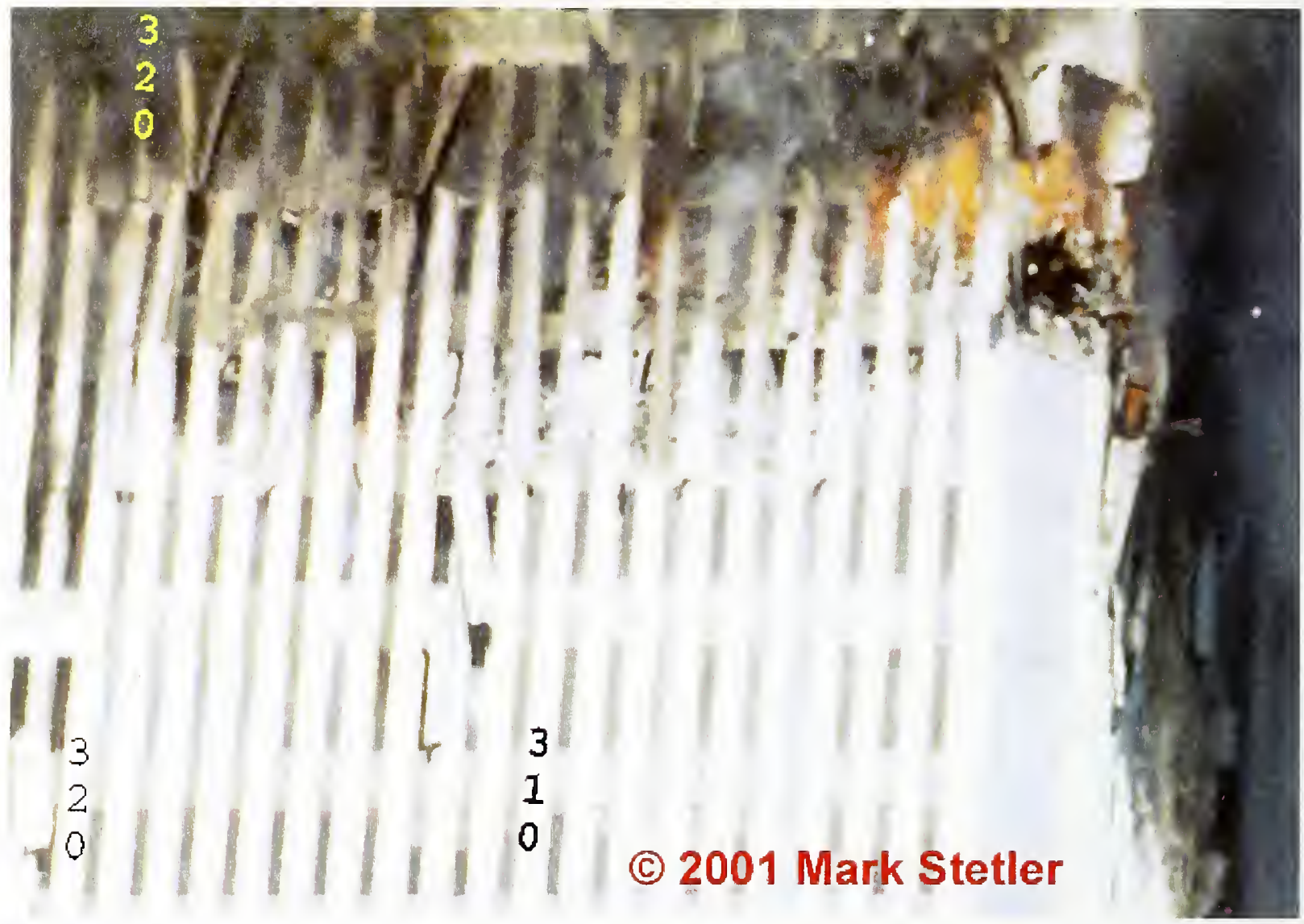

Figure $\mathrm{H}-7$. This photograph is cropped from the image shown in Figure $\mathrm{H}-6$. It was taken on September 11 and shows the east face of WTC 2 at the northeast corner from floor 77 to floor $\mathbf{8 2}$. Note the large piles of debris evident on floor 80 and floor 81 .

\section{H.4.2 Fire Properties}

Photographs and video images have been used to characterize a number of properties relevant to fire growth and spread in the towers as a function of time. Specific properties addressed include whether or not fire and smoke are present and whether windows are still in place. When smoke and/or fire are present, additional details concerning their appearances are documented. A numbered coding system is used to describe these characteristics. The key for this numbering system is shown in Fig. H-8.

\section{H.4.3 Window-by-Window Assessment}

The key in Fig. H-8 is used as the basis for a window-by-window assessment of the towers. The results are coded in three separate data sheets using Microsoft Excel. The floor and window locations are identified using the numbering system described in the last section. Separate files containing the three data sheets are generated for each face of a tower and time analyzed. Figure H-9 shows a portion of such a data sheet describing fires (i.e., sheet one) on the east face of WTC 1 around 9:42 a.m. 


\section{KEY FOR ANALYSIS}

Sheet \#1: Fire Visible
$0 \quad$ No fire
1 Spot fire
2 Fire visible inside
3 External flaming
$9 \quad$ Not visible

Sheet \#2: Smoke
$0 \quad$ No smoke evident
1 "Light smoke"
2 "Heavy smoke"
$9 \quad$ Not visible

Sheet \#3: Windows
$0 \quad$ Window open
1 Window in place
9 Not visible

Figure H-8. The key used to describe observations with regard to fire, smoke, and window breakage in Excel data files for individual windows in the two towers. 


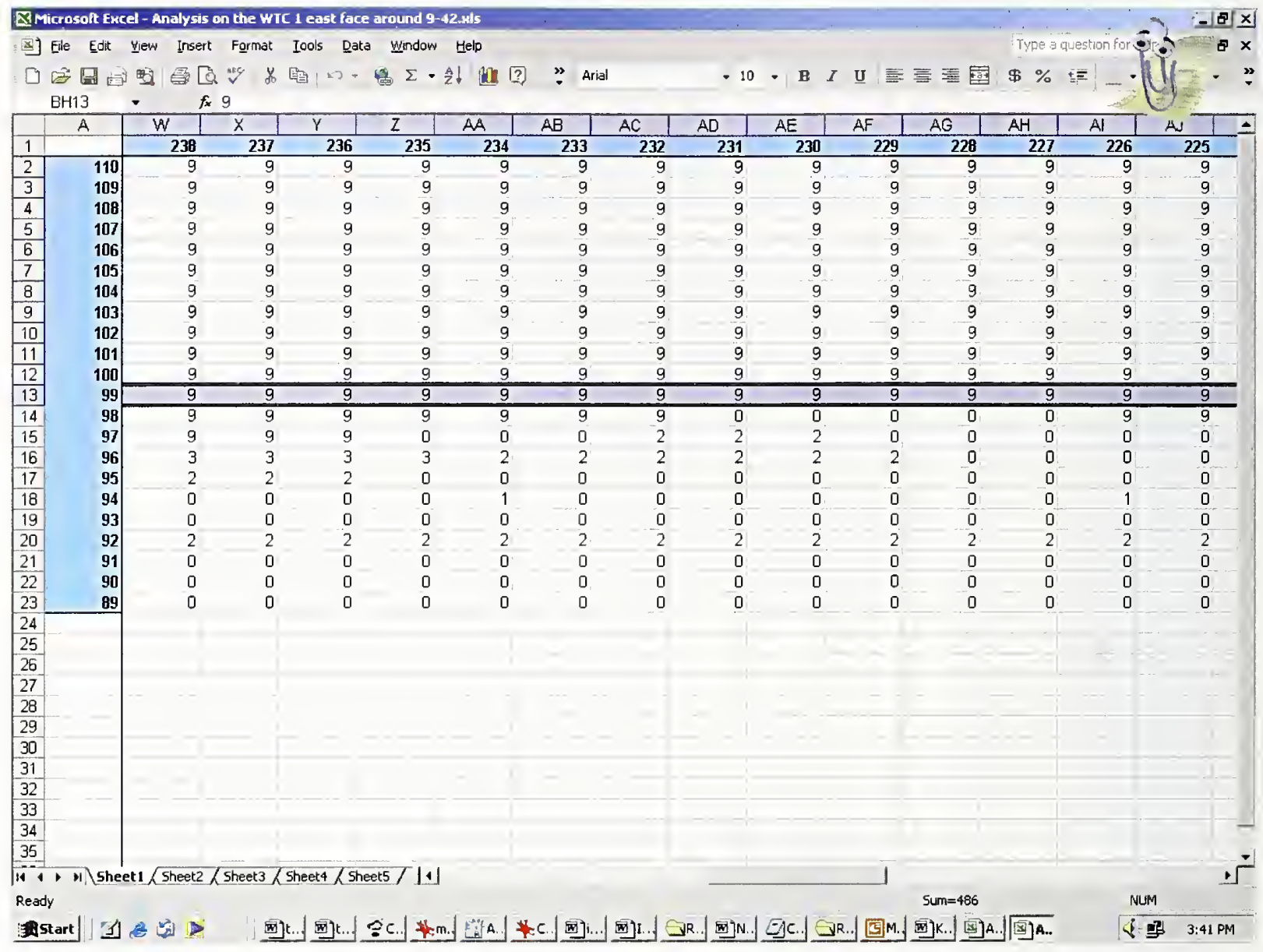

Figure H-9. A portion of the Excel spreadsheet describing fires on the east face of WTC 1 around 9:42 a.m. is shown. The numbers at the left refer to floors, and those at the top are the window numbers.

While the data sheets capture the desired behaviors, it is very difficult to use them to track changes without visualizing the results in some way. Two approaches have been developed for this purpose. The first employs a Web-based system that generates color-coded maps of the results contained in the data sheets. Figure $\mathrm{H}-10$ shows such a map for the fire data included in the data sheet shown in Fig. H-9. The second approach uses the program Smokeview (Forney and McGrattan 2003; Forney, Madrzykowski, and McGrattan 2003) to generate a time-dependent visualization of the results. Smokeview was developed at NIST in order to display the results of fire dynamics calculations. In the current application, it is used to visualize the properties of interest on a three-dimensional representation of a tower façade as a function of time. Because Smokeview allows the point of view to be varied at will, this approach is a powerful means for investigating the temporal behavior of the fires on different faces of the tower. Figure $\mathrm{H}-11$ shows a frame taken from a visualization in which results from the fire and windows data sheets for WTC 2 have been combined. 


\section{||I||||||||||||||||||||||||||||||||||||||||||||||||| 100

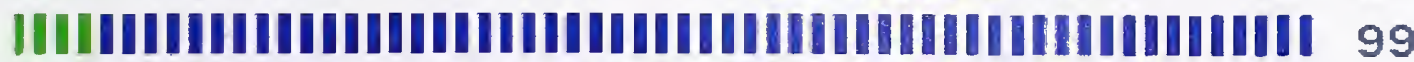

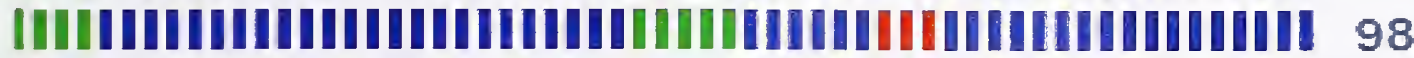 |IIIIIIIIIIIIIIIIIIIIII-UIIIIIIIIIIIIIIII 97

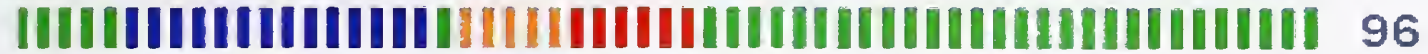

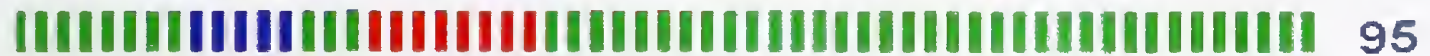

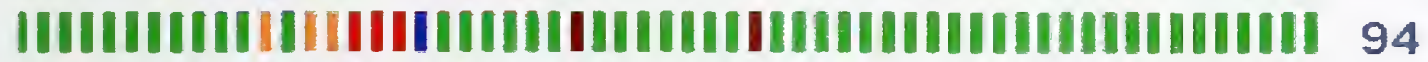

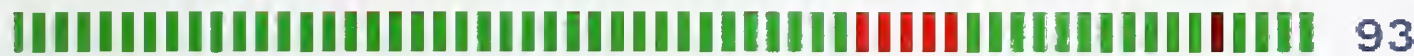

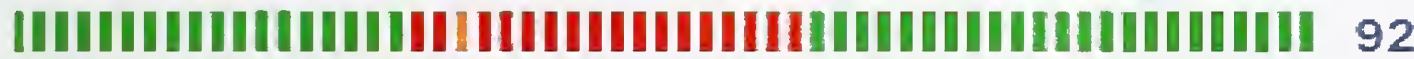

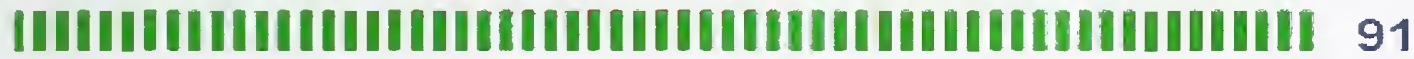 201} 258

Figure $\mathrm{H}-10$. A representation of fires for floors 91 to 100 on the east face of WTC 1 around 9:42 a.m. is shown. Results are taken from the Excel spreadsheet shown in Figure $\mathrm{H}-13$. The color coding is based on the key shown. The color assignments are: 0-No fire, 1-Spot fire, 2-Fire visible inside, 3-External Flaming, and | 9-Can't see.

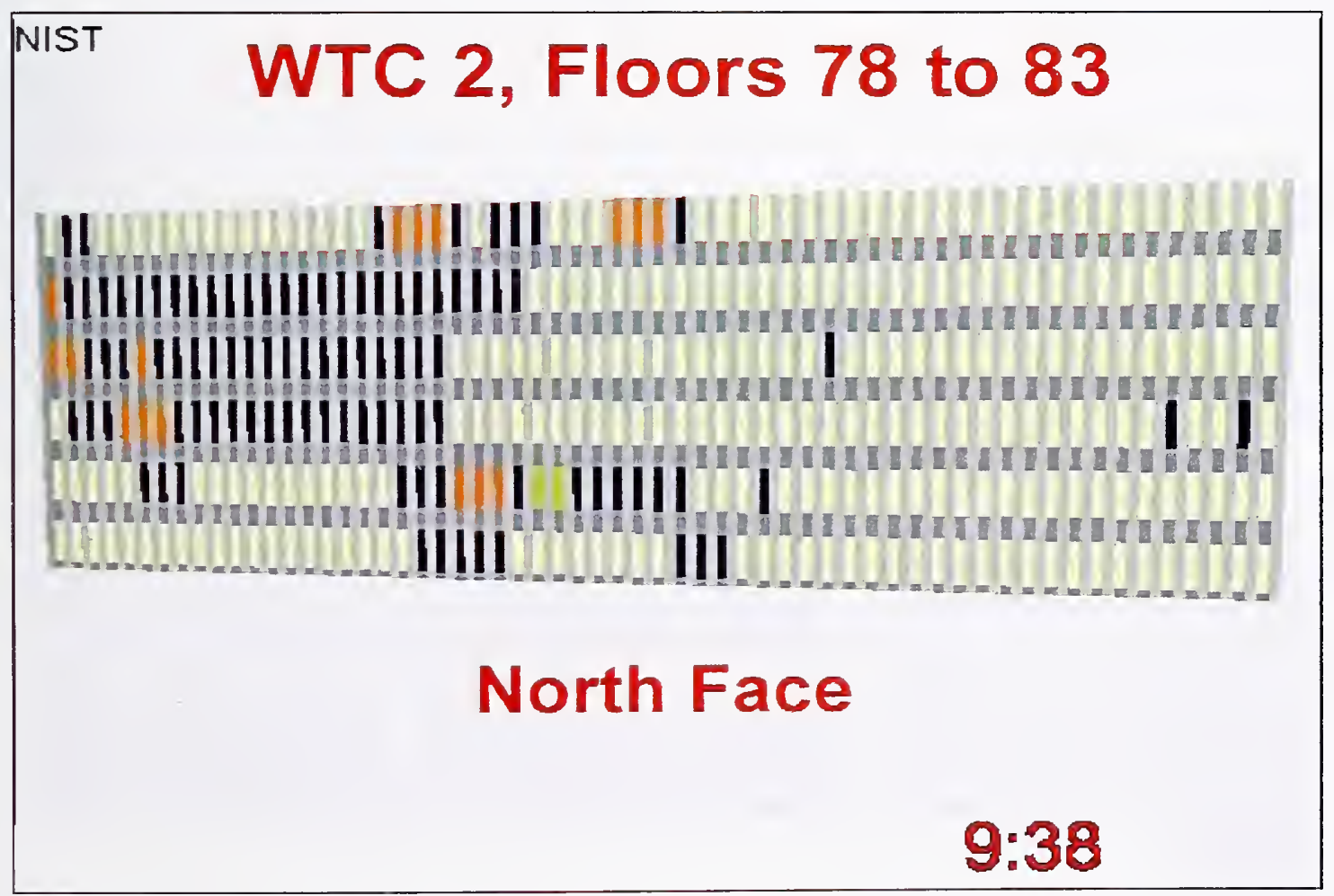

Figure $\mathrm{H}-11$. A single frame from a time-dependent visualization generated by Smokeview is reproduced here. The frame is a three-dimensional representation of the condition of windows and fires on WTC 2 from the time the second tower was struck at 9:02:59 a.m. until it collapsed at 9:58:59 a.m. The color assignments are: - window in place, - missing window, - external flaming, - fire inside, and - spot fire. 


\section{H.5 INITIAL DAMAGE PATTERNS ON WTC 1 AND WTC 2 DUE TO THE PLANE STRIKES}

Close-up photographs and videos have been used to characterize the initial damage to the façades of the towers struck by the two planes along with precise determinations of the locations of the plane strikes. For WTC 2, analysis of videos has also been employed to estimate the speed of the airplane that struck the tower and to show that the tower swung back and forth for several minutes after it was struck. The period of the swinging has also been determined.

\section{H.5.1 WTC 1}

\section{Damage Resulting from Plane Strike}

A detailed drawing of the damage to the steel façade of WTC 1 was included in the FEMA World Trade Center Building Performance Study (McAllister 2002). A careful inspection using photographs and videos in the database confirmed the accuracy of this analysis. Figure $\mathrm{H}-12$ shows a drawing that represents this damage. It is similar to that included in the FEMA report, but it incorporates several minor changes that better reflect the geometry of the north face of WTC 1 in the vicinity of the plane strike.

It was observed that the wing tips and the end of the vertical stabilizer at the plane's tail section damaged the aluminum column covers on the steel façade without cutting through the steel below or completely removing the covers. By inspection it was possible to map out locations on columns where the wingtips and the vertical stabilizer struck the tower. These locations were then transferred to the representation of the damaged steel façade shown in Fig. $\mathrm{H}-12$ and are represented by dashed lines, with wings to the right and left and the vertical stabilizer in the center. The good agreement between the damage pattern and the wing tip locations is evident. It is reported in the FEMA report (McAllister 2002) and widely in the media that American Airlines Flight 11 struck floors 94 to 98 of WTC 1. The dotted horizontal lines on the left side of Fig. H-12 indicate the locations of concrete floors. It can be seen that while the tip of the left wing of the aircraft struck very close to the base of floor 94, the wing end marked column 153 at the very top of floor 93. It is evident from the figure that the right wing actually struck well up on floor 99 on column 109. The impacted floors therefore range from floor 93 to floor 99.

\section{Fireballs and Missing Windows}

Additional insights into the initial damage inflicted on the towers by the plane strikes can be obtained by considering locations where fireballs are observed immediately following the plane strikes as well as locations where windows are missing. Videos and photographs recorded during and immediately following the plane strike on WTC 1 show that significant fireballs formed at the plane strike location on the north face, as well as near the center of the east face and on the western side of the south face. Figure H-13 compares window damage for the four sides of WTC 1 immediately after the plane strike. The floors shown extend from 91 to 100 . The missing windows on the north face are consistent with the plane strike location and strike angle. The plane struck very close to the center of the face. Interestingly, the damage on the east and west faces appears to be asymmetric with a much higher number of windows missing on the east face than on the west. This observation is consistent with the formation of a fireball on the east side of the tower and not on the west side. Areas obscured by smoke are also much larger on 


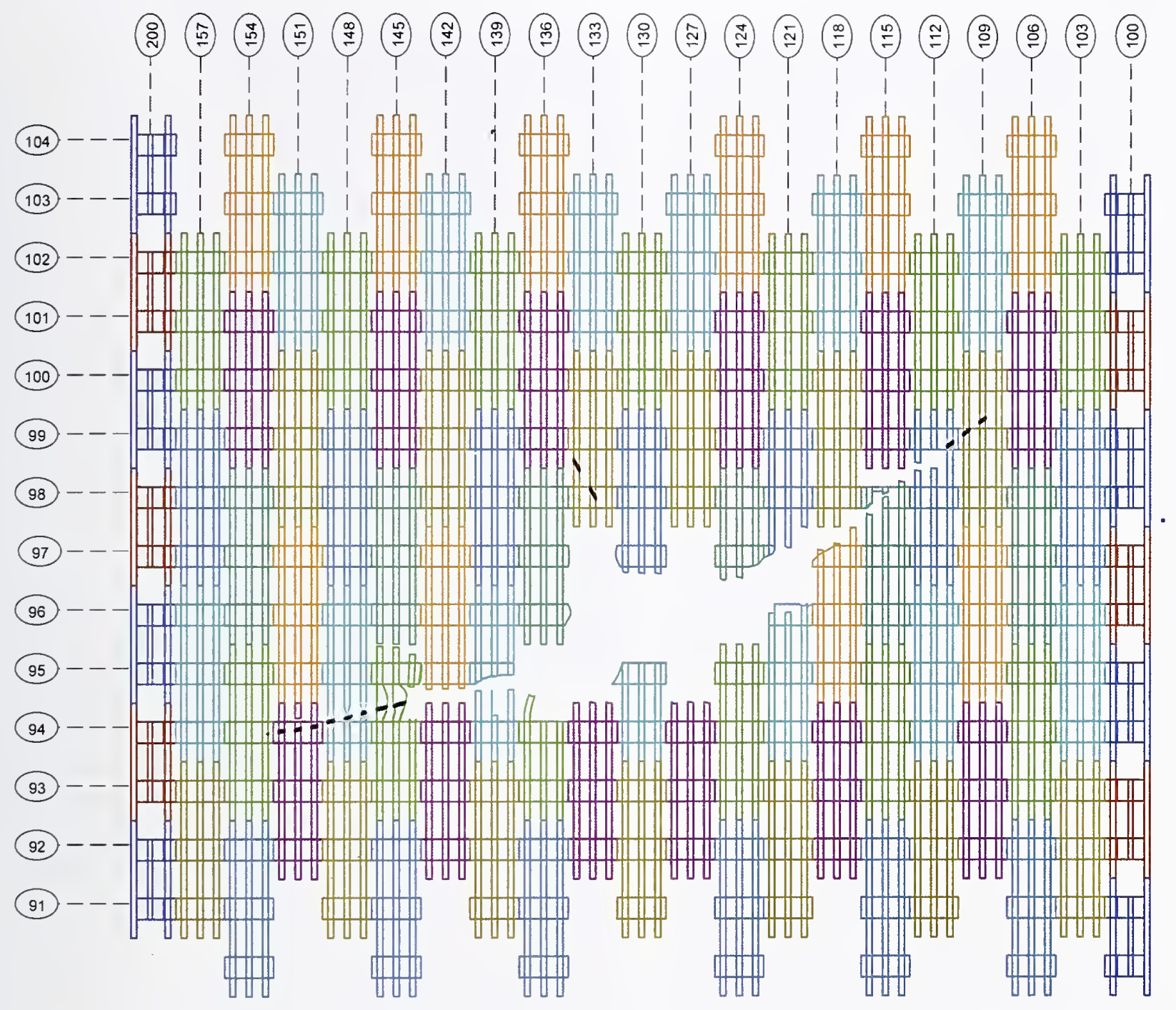

\section{Figure $\mathrm{H}-12$. A drawing of the damage to the steel façade of WTC 1. The dark dotted lines show locations where the airplane wings and vertical stabilizer marked the aluminum cladding on columns.}

the east side suggesting that more fire is present on this face as well. An asymmetry is also apparent on the south face where only a single window is missing on the east side while numerous windows are missing and significant smoke is present on the west side. Taken together, these observations suggest that debris and fuel from the airplane as well as any building materials and contents tended to pass straight across the building on the west side, while material on the east side was somehow reflected and more heavily damaged the east face. 


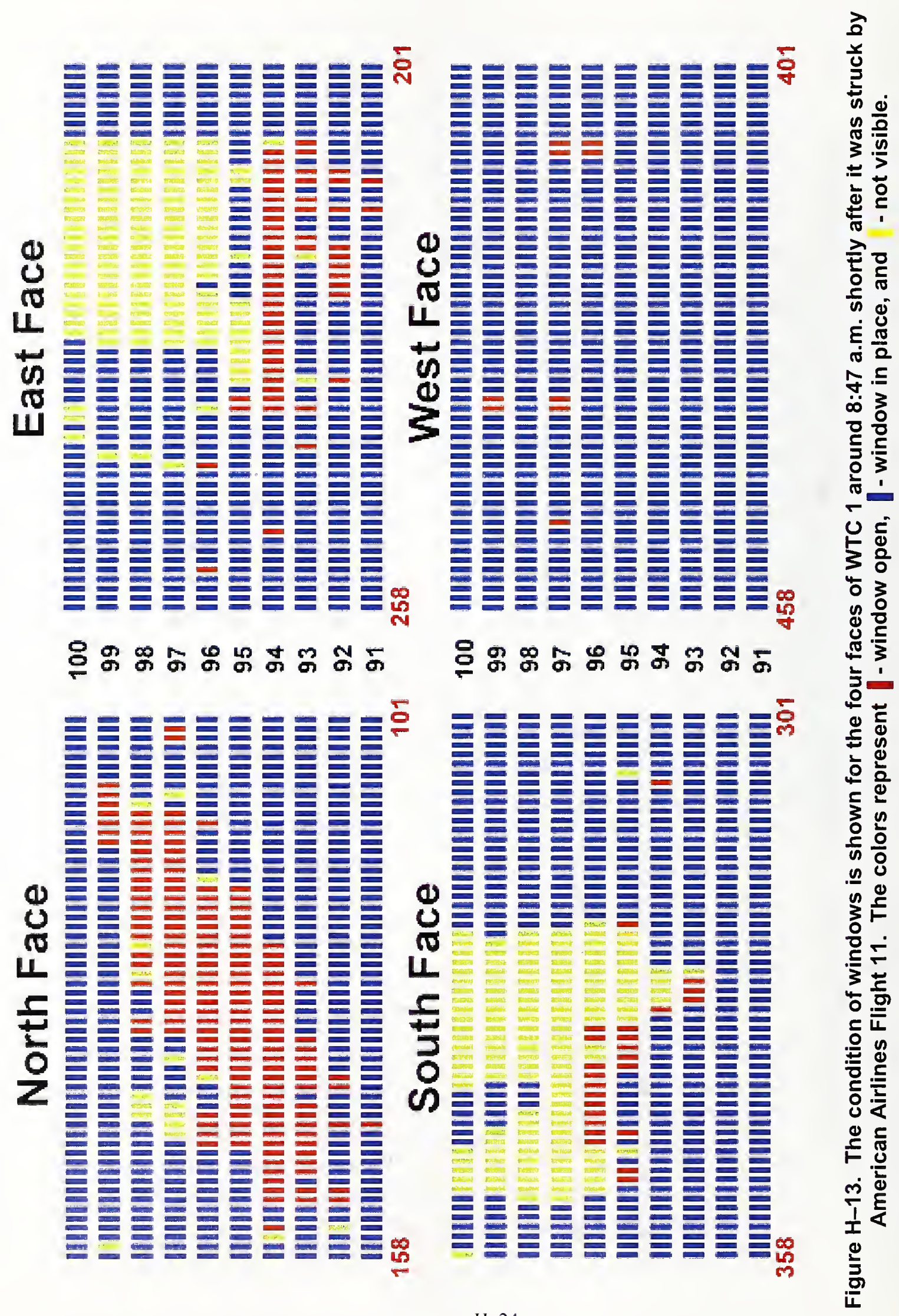

H-24 


\section{Panel Section in Street}

A photograph supplied by the NYPD provided additional details with regard to the initial damage suffered by WTC 1. Figure H-14 shows a full three-story three-column-wide steel panel section lying on the corner of Cedar Street near its intersection with West Street. This location is to the south of and roughly $210 \mathrm{~m}$ from the south face of WTC 1 . The photograph was taken prior to the collapse of either tower. Closer inspection shows that there is an aircraft wheel embedded in one of the windows. The most likely source location for this panel section has been identified as being near the center of the south face of WTC 1 (i.e., columns 329 to 331) and extending from the middle of floor 93 to the middle of floor 96. This conclusion remains tentative since, as indicated in Fig. $\mathrm{H}-13$, the area is obscured by smoke in all of the close-up photographs of the area in NIST's possession. If the location is identified correctly, the wheel is stuck in window 95-329.

\section{H.5.2 WTC 2}

\section{Calculation of Plane Speed}

One of the videographers who provided material to the Investigation filmed from the top of his apartment building located to the east of the WTC complex. His camera was located on a tripod so that the images are very steady. One of the events he captured was United Airlines Flight 175 as it approached WTC 2. Figure $\mathrm{H}-15$ is a series of cropped frames captured from this video that show the plane approaching the building.

The images included in Fig. $\mathrm{H}-15$ have been used to determine the speed of the plane as it approached the tower. This is done by identifying the locations of the nose and tail of the airplane relative to a fixed point defined to be the point on the frame where the plane passes out of sight behind the corner of the building. The plane is very nearly level relative to this point, so it is appropriate to simply count the number of picture elements, pixels, between this location and the two measurement points on the aircraft.

This analysis, which presumes that the aircraft at this time travels in a straight path such that the nose and tail pass through the same point in space, has the advantage of being independent of the orientation of the flight path with respect to the line of sight of the observer.

Figure $\mathrm{H}-16$ shows the locations of the two points as a function of time. Using linear least squares curve fits, the exact relative times when the nose and tail pass the reference location are estimated. The difference between these two times is the period required for the entire length of the aircraft to pass the reference location. The result is $0.1939 \mathrm{~s}$. Since the length of the plane is known to be $155.0 \mathrm{ft}$, the speed can be determined simply by dividing this length by the passage time to give $155.0 \mathrm{ft} / 0.1939 \mathrm{~s}=799 \mathrm{ft} / \mathrm{s}$ $=545 \mathrm{mph}$. An uncertainty estimate based solely on the uncertainty in the determined time difference yields a value of $\pm 18 \mathrm{mph}$ with 95 percent confidence. 


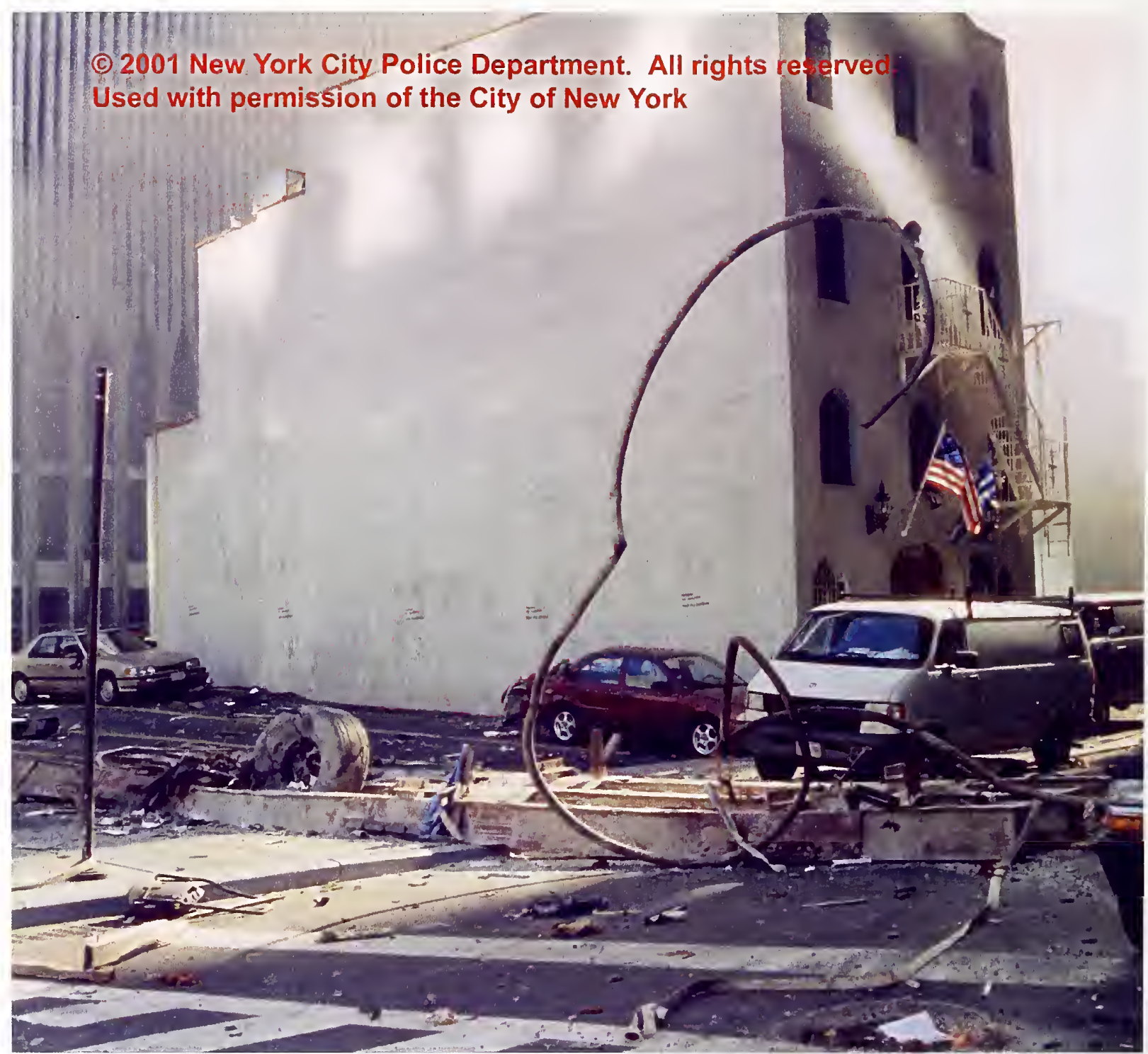

Figure $\mathrm{H}-14$. Photograph showing a full panel section lying in Cedar Street near its intersection with West Street. An aircraft wheel can be seen imbedded in one of the windows. The building behind the panel is Saint Nicholas Greek Orthodox Church and the lower section of WTC 2 can be seen across Liberty Street.

Note that the airplane speed and uncertainties are slightly different than listed in an earlier report (NIST 2003) due to a correction of the plane length to reflect the actual distance between the nose and the end of the body at the rear stabilizer and a math error in the uncertainty calculator. Uncertainties associated with aircraft motion that are not aligned with the aircraft body are judged to be less than the uncertainties in plane passage time.

\section{Observation of WTC 2 Sway Following the Plane Strike}

Close examination of the video revealed a perceptible movement of WTC 2 after it was struck by the aircraft. The building rocked back and forth much as a pendulum for at least 4 minutes. Image 

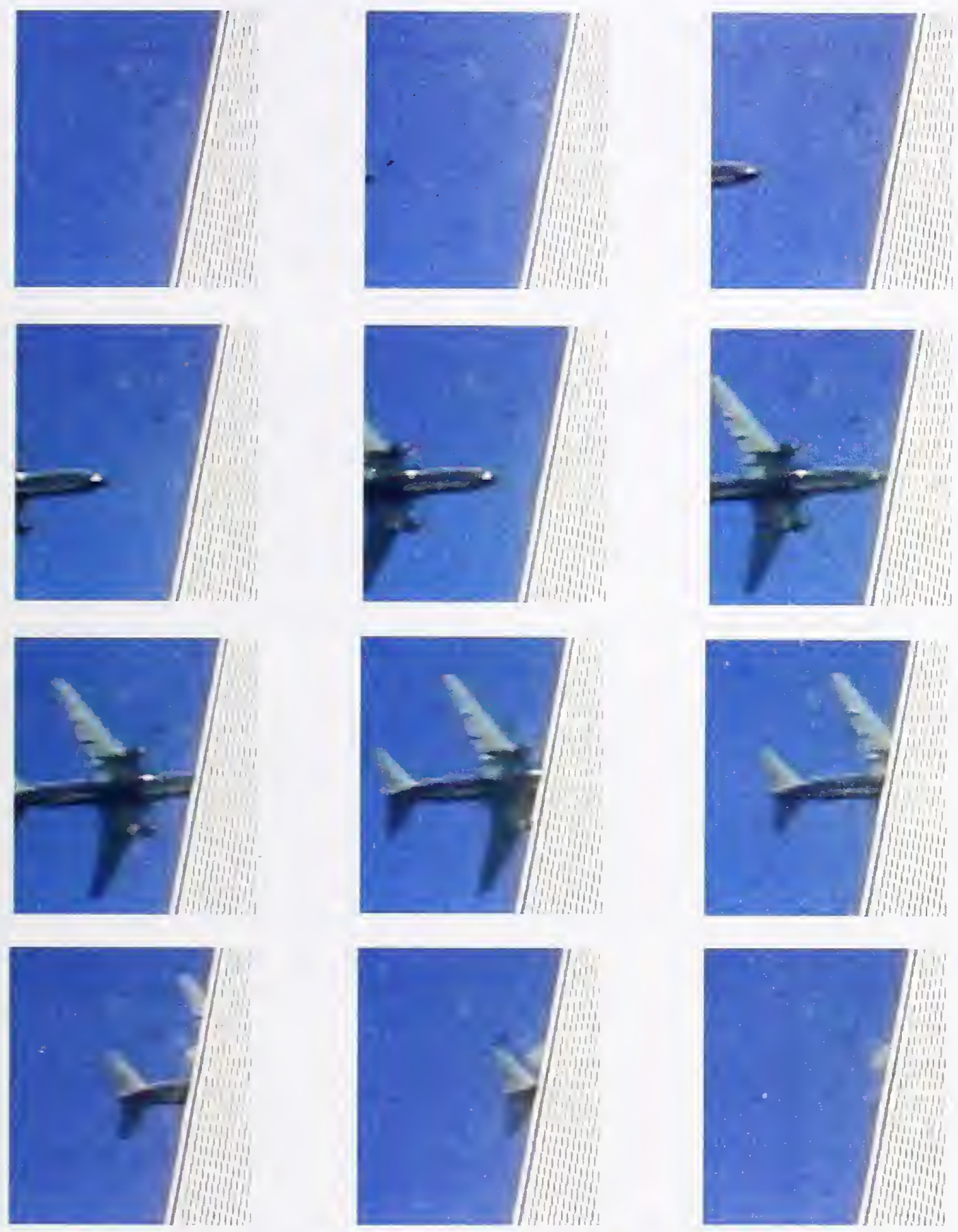

\section{(C) 2001 Scott Myers}

Figure $\mathrm{H}-15$. Series of sequential cropped frames taken from a video shot on September 11, 2001, showing the plane approaching WTC 2 . The frames, ordered from left to right and top to bottom, are separated by $33.3 \mathrm{~ms}$. 


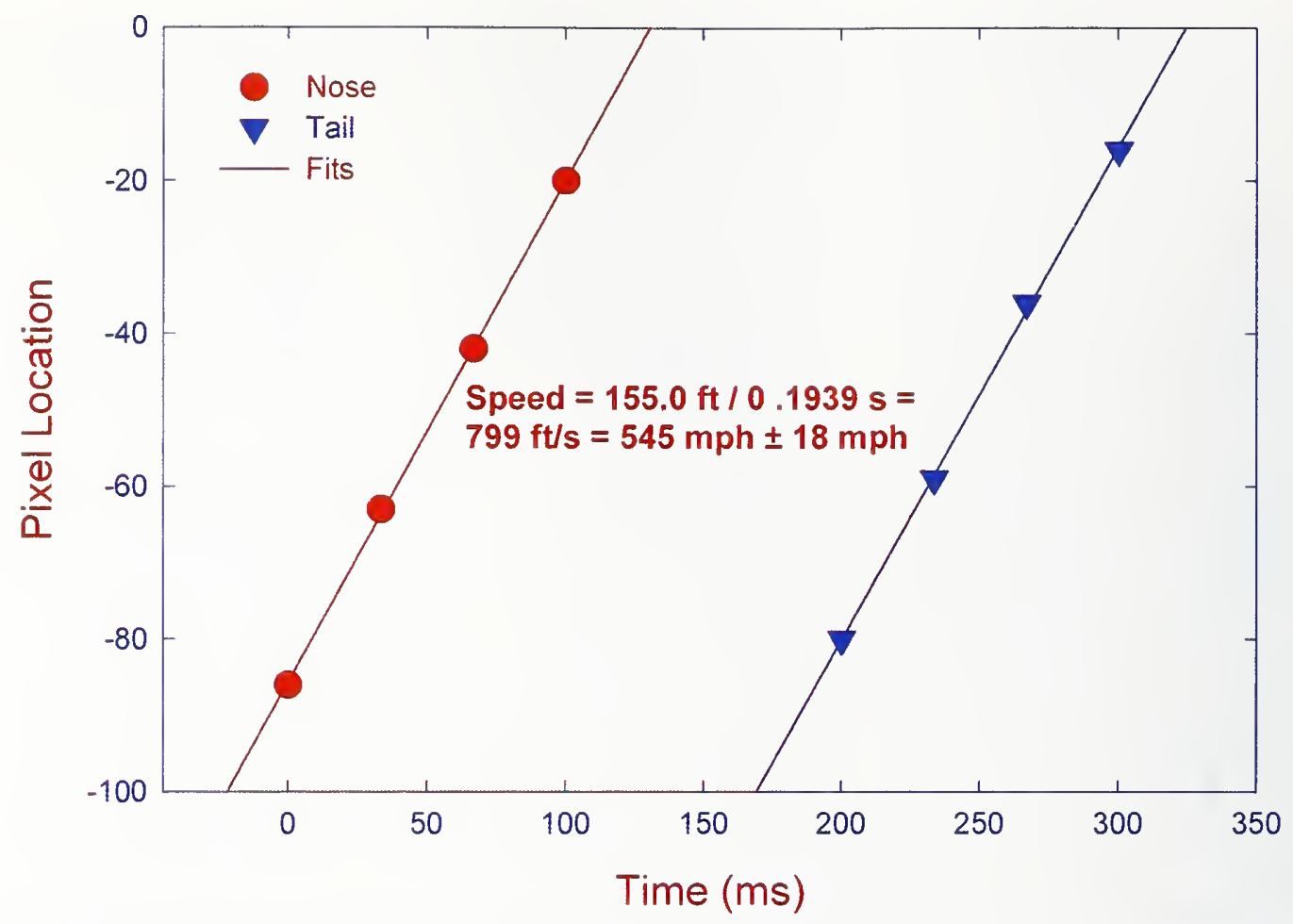

Figure $\mathrm{H}-16$. Plots of pixel locations for the nose and tail of the plane that struck WTC 2 as a function of time taken from the images shown in Fig. $\mathrm{H}-15$. Straight lines are the results of linear least squares curve fits to the data. Extrapolation of the lines to pixel 0 allows the time for the passage of the plane to be calculated.

analysis was used to enhance this motion and estimate the period required for the building to sway through one complete cycle. This was accomplished by creating a new video in which a single frame just prior to the plane strike was subtracted from subsequent frames. In this way, small differences between images can be identified. If the image is unchanged from the initial frame, the result should be a black image, but any changes in location or color will appear in the difference video. When this approach was applied to the video, a region of windows was observed on the building that seemed to appear and disappear. Figure $\mathrm{H}-17$ shows several frames of a composite video formed by overlaying half frames of the original video and the difference video. In the initial frame (time $=0.0$ seconds), the plane has not yet appeared and the difference frame is black. In the next frame (time $=10.7$ seconds), the plane is approaching the building. The plane is evident in the difference frame since it represents a change in the frame. WTC 2 is still dark except near the top where changes due to smoke movement are apparent. In the third frame (time $=11.3$ seconds), the plane has struck the building and dramatic changes in the appearance of the building façade in the difference frame occur. Careful inspection shows what appear to be curved lines running across the face of WTC 2 . These curves result from an interaction between the straight lines formed by the windows on the tower and the straight lines of picture elements that make up the detector in the digital video camera. This well-known behavior is called the moiré effect. The moiré effect also provides a sensitive approach for determining the displacement of the building. Such an analysis is in progress and will be reported at a later time. 


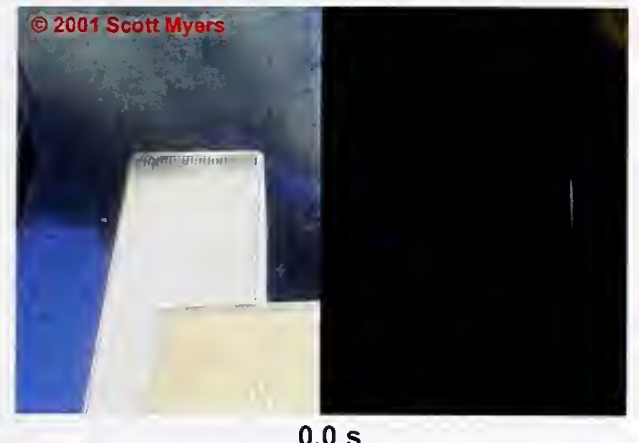

$0.0 \mathrm{~s}$

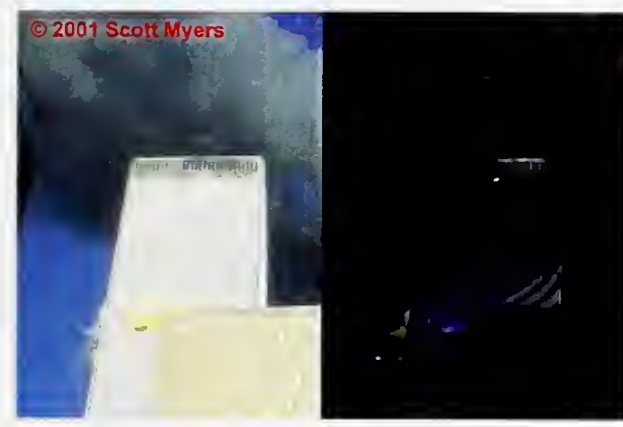

$11.3 \mathrm{~s}$

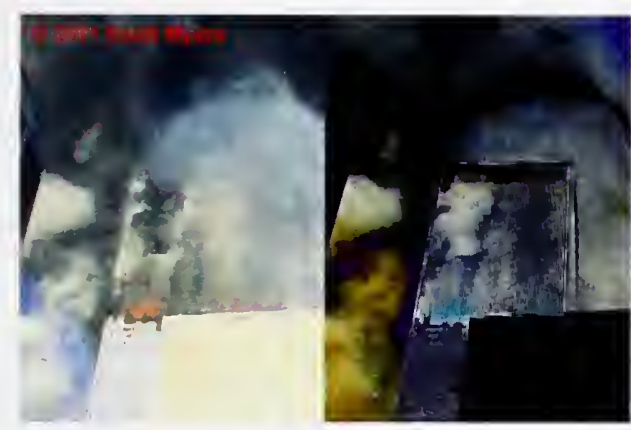

$30.9 \mathrm{~s}$

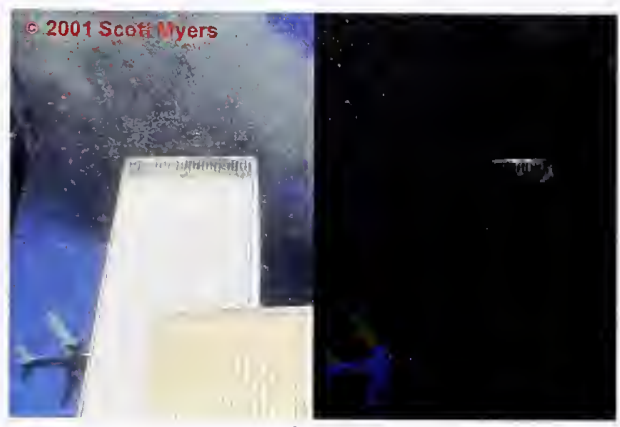

$10.7 \mathrm{~s}$

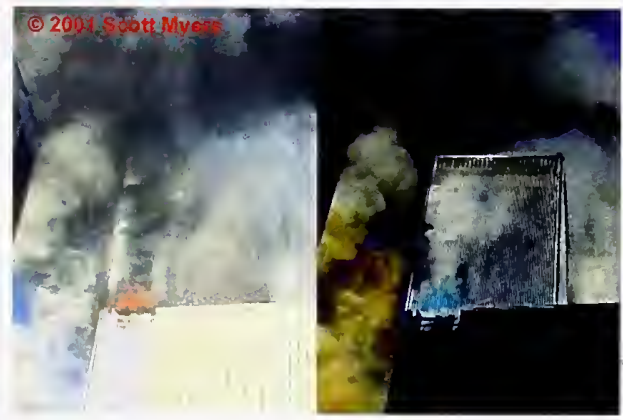

$28.0 \mathrm{~s}$

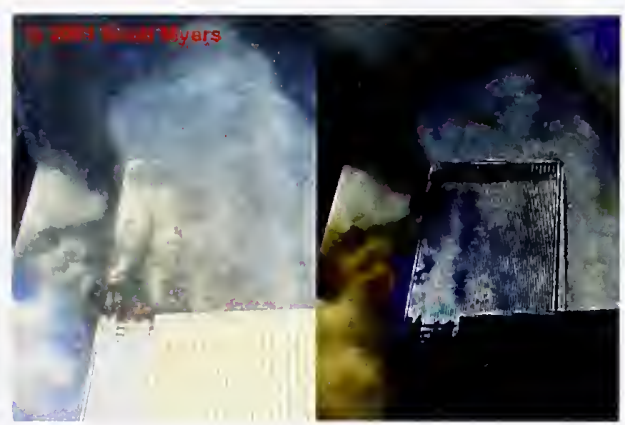

$33.5 \mathrm{~s}$

Figure H-17. Frames from a composite video are shown. The half frames on the left are taken from a video showing the plane strike on WTC 2, while the half frames on the right are generated by subtracting a frame recorded prior to the plane strike from all subsequent frames in the original video. Times refer to the period since the start of the difference video.

Following the plane strike, areas of the tower face above the strike floors become hidden by smoke, and it is difficult to see the moiré patterns in the difference frames. However, the area of the tower below the strike floors to the left of the building in the foreground continues to show a distinct difference pattern because it is not obscured by smoke. This pattern is apparent in the fifth frame (time $=30.9$ seconds). On the other hand, frames 4 and 6 (times $=28.0$ seconds and 33.5 seconds, respectively) have been chosen because they are near null points, and the area appears dark in the difference frame because the location of the building is essentially unchanged from its position before the plane struck. When the video is 
played, the moire patterns in this area of the tower face alternately appear and disappear in the difference video.

Because the absence of color is easiest to identify, it is straightforward to determine times when the null points occur in the difference image. Figure $\mathrm{H}-18$ shows a plot of time versus null point number obtained from the difference video. The points fall on a straight line having a slope of 5.647 seconds \pm 0.008 seconds ( 95 percent confidence interval). Because the building passes through a null point twice during a single full oscillation, the period required for a single oscillation is 11.3 seconds.

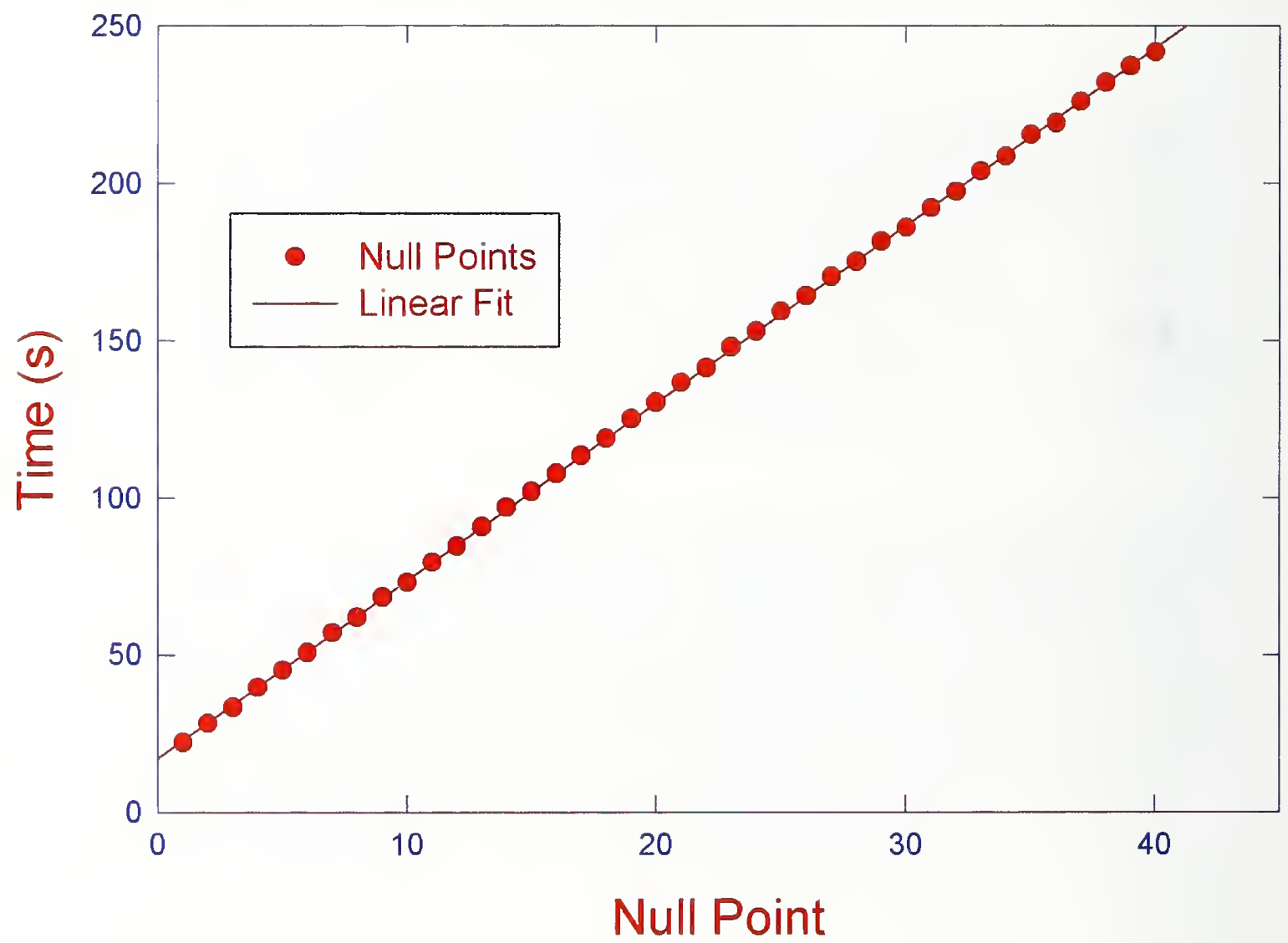

Figure $\mathrm{H}-18$. The time when null points are observed in the difference video following the plane strike on WTC 2 are plotted versus the null point number. The points fall on a very good straight line having a slope of 5.647 seconds \pm 0.008 seconds.

The measured oscillation period is consistent with measurements that are available from WTC 1 that yielded periods of 10.9 seconds in the east-west direction (averaged over a 9-year period that ended in 1993 ) and 11.6 seconds in the north-south direction (averaged over a 14-year period that also ended in 1993). The cores of the two towers were oriented perpendicular to each other so the motion monitored here should be comparable to the east-west direction of WTC 1. 


\section{Damage Resulting from the Plane Strike}

The results of an analysis of the damage to the steel façade of the south face of WTC 2 are provided in Fig. H-19. Much of the steel damage pattern is revealed, but it should be noted, as indicated, that a portion of this face on the east side of the plane strike location was constantly obscured by smoke, and the detailed pattern could not be discerned. The' FEMA report (McAllister 2002) also includes a figure describing the damage to the steel façade inflicted by United Airlines Flight 175. The pattern in Fig. H-19 differs somewhat from that provided in this earlier study. Some inconsistencies in façade dimensions have also been corrected in the current version.

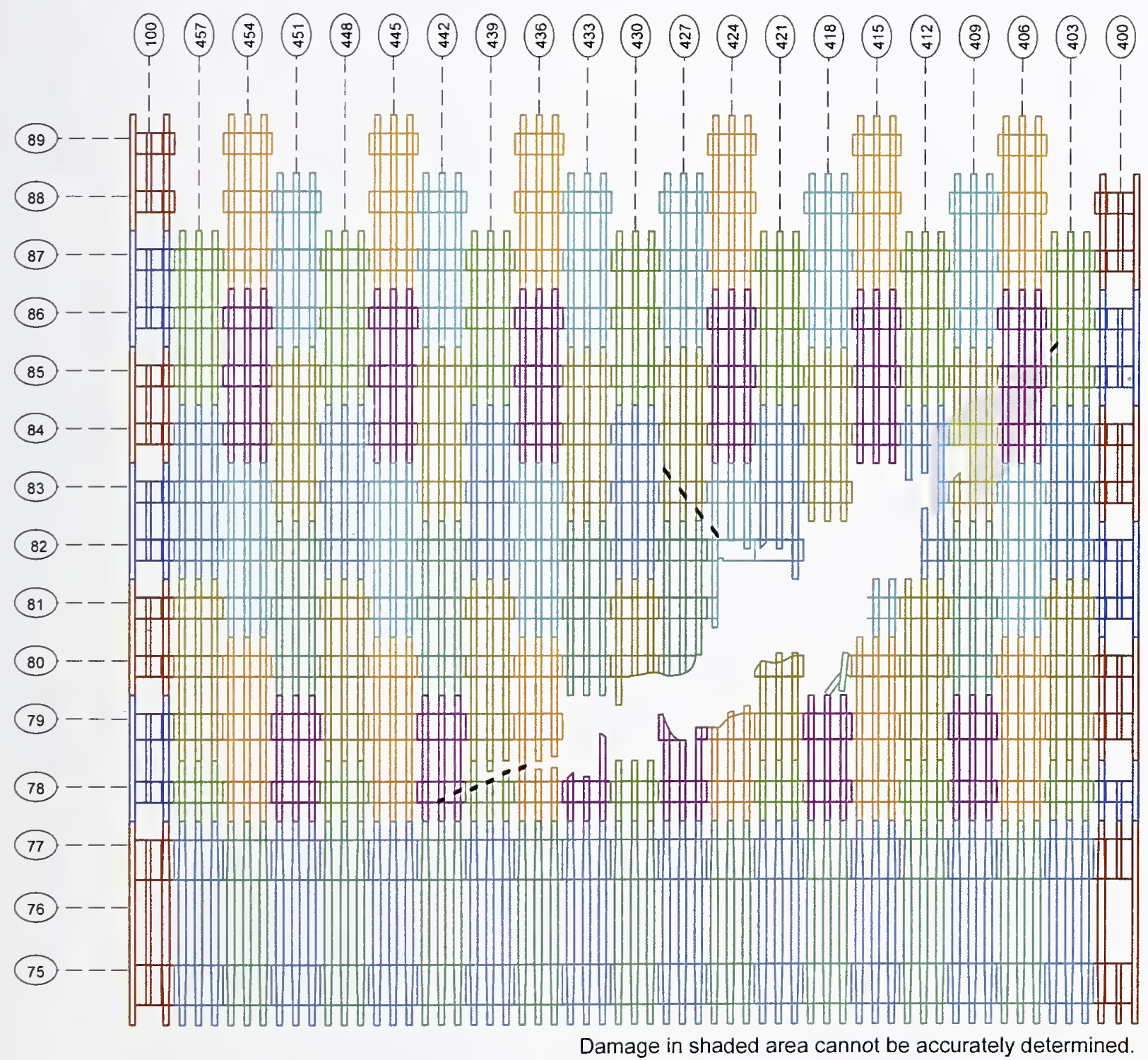

Figure H-19. A drawing of the damage to the steel facade of WTC 2. The dark dotted lines show locations where the airplane wings and tail marked the aluminum cladding on the columns. The area shaded in gray was hidden by smoke and could not be observed. 
As was true for WTC 1, in areas of the façade struck by the wing tips and the upper portion of the vertical stabilizer the aluminum covering was marked, but the aluminum covers were not removed and the steel was not cut through. Measurements for the location of the left wing tip were mapped out as shown in Fig. H-19. As already noted, the area at the end of the right wing was obscured by heavy smoke. However, there were brief periods when the location of the last column struck by the wing tip could be observed. This location is indicated on column 404 of floor 95 in Fig. H-19. The center of the plane strike is clearly located towards the east side of the face. The left wing mark extends to the bottom of the spandrel located below floor 78. The actual location of the concrete floor is well above this point, which means the lowest point struck lies on floor 77. Thus, the plane strike location on WTC 2 extends from floor 77 to floor 85. This can be contrasted to the FEMA study (McAllister 2002) and most media sources that report the floors struck extended from floor 78 to floor 84 .

\section{Fireballs and Missing Windows}

Intense fireballs were observed on the south, east, and north faces of WTC 2 following the plane strike. Figure H-20 compares missing windows on floor 77 to floor 86 for the four faces of WTC 2 shortly after the plane struck at 9:02:59 a.m.

The distribution of missing windows on the south face traces roughly the outline of the plane strike, with missing windows increasing in height from left to right. Recall that a portion of the east side of this face could not be observed due to smoke obscuration. The analysis indicates that a very large number of windows were removed on the east face by the collision and subsequent fireball. This is particularly true on floor 80 to floor 82 . Photographs and videos show that extensive areas of the aluminum covering the façade and holding windows were removed, exposing the steel panels, as a result of the plane strike and fireballs. This damage is much more extensive than observed on the east face of WTC 1, consistent with the plane strike occurring closer to this face. In contrast to the extensive damage on the east face of the tower, no missing windows were found on the west face.

A large number of windows are also missing on the north face of WTC 2. A substantial area of the aluminum façade was also removed during the plane strike and subsequent fireball. The missing windows on this face almost appear to be a mirror image of the south face with damage towards the center being on lower floors than on the eastern edge. This suggests that a great deal of debris passed through the entire length of the building. This hypothesis is supported by close up images that show large piles of debris on the east side of the north face on floor 80 and floor 81, and on floor 79 near the center of the face. Figure H-21 includes a photograph showing these debris piles. Recall that piles of debris were also evident on floor 80 and floor 81 on the north side of the east face (see Fig. H-7).

\section{H.6 FIRE BEHAVIORS IN THE TWO TOWERS}

Analysis of the fire spread in the towers is ongoing as this update is being prepared, but sufficient information is available to allow some of the fire characteristics to be described. 
(1)

0

iv

+ 으를를르르르를

$\varphi$ 르를를르르르를

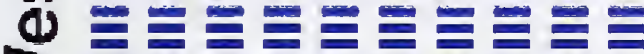

2 드르르르르르르를

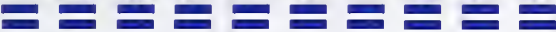

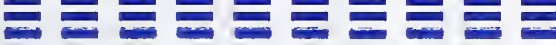

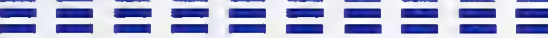

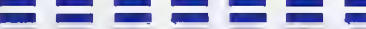
을으를르르를

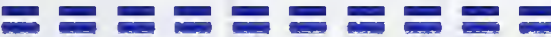
으르를르르르를

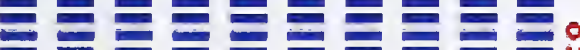

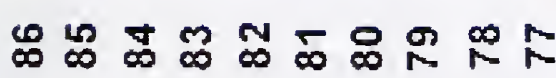

0

U

त)

u

$\frac{5}{2}$
$\frac{3}{0}$

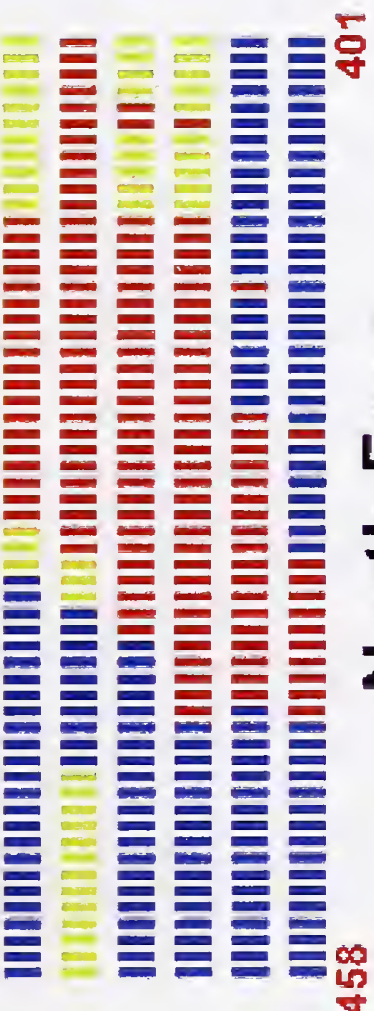

5
0
0
1
4
1
1
0

()

U

(ర)

山

o

யบ

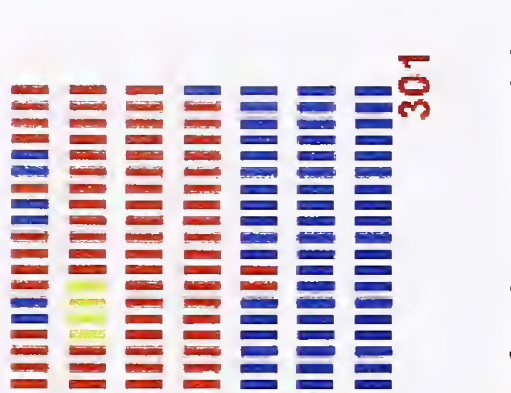

ลิำ 눈

(⿻)

$3 \frac{0}{5}$

$\pm>$

边 은

ț

党 $\frac{2}{\pi}$

$\therefore=2 \mathrm{E}=2 \mathrm{E}$

$= \pm \equiv \equiv$

드르르르르를

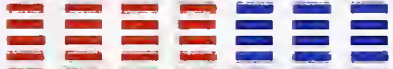

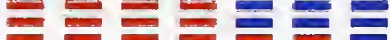

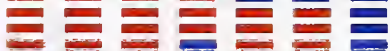

드르리를

$= \pm \equiv \equiv \equiv$

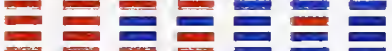

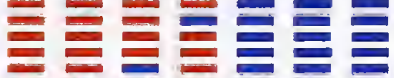

프르르를

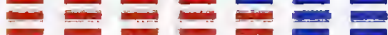

드를를 를

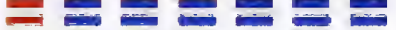

$=-2=20$

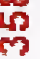

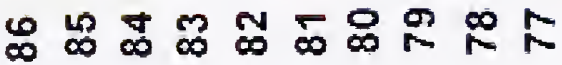

$+$

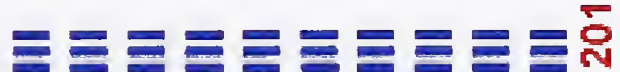

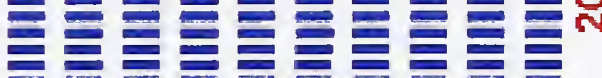

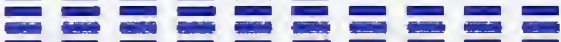

르를르르르르르를

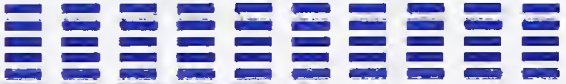

를ㄹㄹㄹㄹㄹㄹ를

a) $-2=-2=$

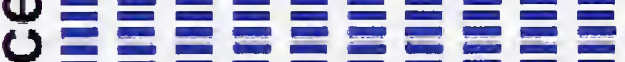

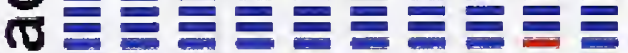

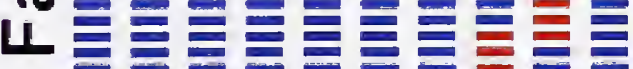

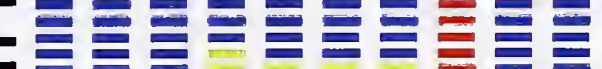

드를

$=\equiv$

$\mathrm{O}=\mathrm{E}=$

$=2$

르르르르르를 을

르르르르르를

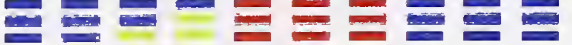

프르를

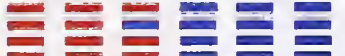

$= \pm \equiv$

$-\sum \equiv \equiv \equiv \equiv \equiv$
ह่

is $\frac{1}{2}$

ก.

के 3

응

동 을

은

$N=$

$\cup$

ㄴㅇㅇ

능

03

엉 응

必

文?

Ð

동

는 잉

5 인

3 n

ต음

.

号是

을

둔

능츨

등 은

는

등옹

는

탄

它

I

은

요 


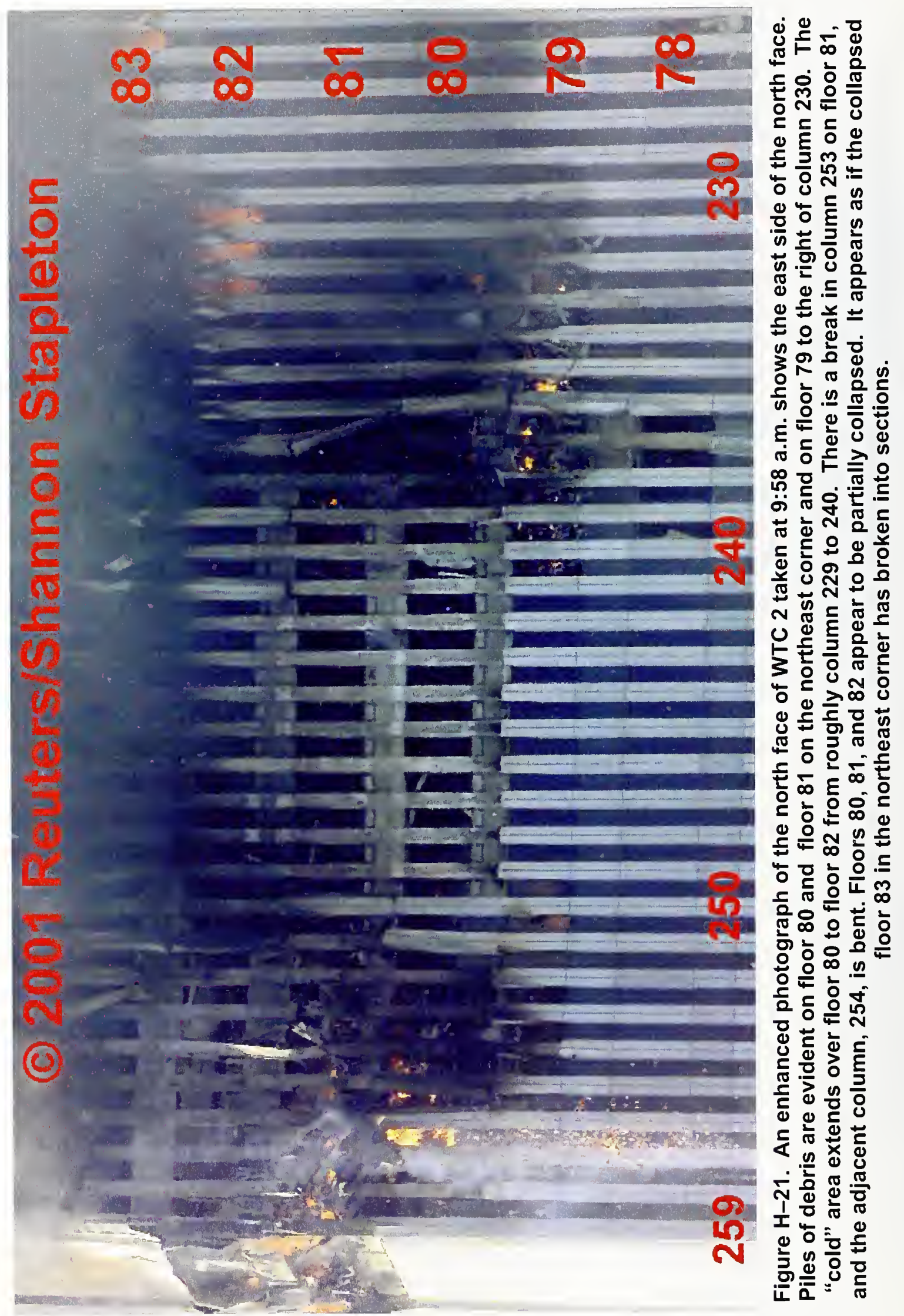




\section{H.6.1 WTC 1}

It has already been mentioned that substantial fireballs formed on the north, east, and south faces immediately following the plane strike. A brief period of intense burning from openings on these faces was observed after the fireballs dissipated, but in a short period (on the order of 60 seconds) the fires seemed to "damp down" and very little flame and only light smoke was evident from the outside. This period of light burning lasted several minutes before fires began to reappear.

Rapid early fire growth was observed on the east side of the north face on floor 96 and floor 97, the center of the east face on floor 94 and floor 97, and the western side of the south face on floor 96. Even though relatively little initial damage was sustained by the west face, heavy smoke followed shortly by flame appeared around window 97-437 at 8:55 a.m. After this time, a very rapid fire spread was observed across the west face on this floor. Within a couple of minutes, over half of the windows were emitting smoke, and flames were visible in many. Even though floor 92 was not directly struck by the airplane, fire appeared on the east side of the tower on this floor shortly after 9:00 a.m.

Following the initial development of large fires, fire spread continued until WTC 1 collapsed around 10:28 a.m. At times the fires displayed the systematic, relatively slow spread expected for fire growth in a typical building. For instance, after the initial rapid growth phase, fires on floors 92, 94, 96, and 97 on the east face began to move deliberately toward the south. As they spread, the fires would burn intensely at a given location for a while before dying down. As a result, these fires developed the appearance of a wave moving slowly across the building.

There were also certain times and locations during which fire appeared to spread quite rapidly. Some of these episodes were clearly connected with rapid fire growth and likely flashover in rooms. During the first half hour, significant fires were observed toward the centers of floors 92, 94, 96, and 97 on the east face that were spreading towards the north. Each of these fires eventually reached a certain point where further fire spread was inhibited for many minutes. A review of building plans showed that walls of offices or meeting rooms were presented at the locations where fire spread was inhibited.

Apparently, these walls served as effective fire breaks that protected against further fire spread. However, for each of these floors fire and smoke eventually appeared at one of the windows beyond the walls, and after one of these windows was broken fire growth was extremely rapid and robust across the remaining windows. These observations are consistent with the occurrence of flashover within an enclosed space.

At other times, unusually rapid fire growth apparently occurred in areas that are believed to have been relatively open and not constrained by walls. One of these episodes occurred around 9:54 a.m. on the north face. Fire suddenly appeared on floor 96 , a location to the west of the damage inflicted by the airplane. Within a very short period of time, fire could be seen in roughly 10 windows covering a distance of more than $30 \mathrm{ft}$.

Another example of very rapid fire growth appeared to take place on floor 98 . In the early period of the fire, this floor did not appear to be heavily involved, and this remained true for quite a while. However, after 9:30 a.m., fire began to appear on this floor and by 10:00 a.m., fires were observed over significant lengths on all four faces of the tower. 
One of the more unusual fire spread episodes in WTC 1 occurred just after the collapse of WTC 2 around 9:59 a.m. Within a couple of minutes, a large intense fire suddenly appeared on the south side of the west face on floor 104 in an area well above any other apparent fire. This unusual jump in fire location is difficult to explain, but is likely associated with vertical shafts located in the core of the tower.

For most of the time following the plane strike, no fire was observed on any of the floors on the south face over lengths extending from the eastern edge of the tower to near the center of the face. Fires were not observed in this region of the building until around 10:00 a.m. By the time this tower collapsed roughly 25 minutes later, intense fires extending over significant lengths of the originally uninvolved area were burning on floor 94 to floor 98 in this area.

A final example of rapid fire spread and growth in WTC 1 was described previously in the May 2003 Progress Report for the Investigation (NIST 2003). In this case, a line of smoke appeared suddenly over a significant length of floor 92 on the north face of WTC 1 at 10:18:48 a.m., or roughly 9 minutes before the collapse of the tower. Puffs of smoke were observed simultaneously on the north face from floors 94 , 95, and 97. More isolated puffs were seen at the same time from floor 92 and floor 95 on the west face and from floor 92 on the south face. Very shortly (seconds) after the appearance of the smoke, a localized fire on floor 95 to the west of the plane strike location grew very rapidly and flames erupted from windows. Following the smoke release, a large fire began to spread rapidly across the western side of floor 92 on the north face. Previous to the appearance of the smoke, only small fires were evident on this floor. By the time the tower collapsed, this fire had spread across most of the floor and had reached the western wall. This fire was responsible for the large burst of flame from the north face observed when this tower collapsed.

\section{H.6.2 WTC 2}

The fire behavior observed in WTC 2 was qualitatively different than occurred in WTC 1 . Intense fireballs were created by the released jet fuel on the south, east, and north faces immediately after the airplane struck the building. As observed for WTC 1, the fireballs were followed by a brief period (on the order of a minute) of intense flaming from windows over a large area of the building. Most of these flames then "damped down" as observed in WTC 1, but two regions of intense burning remained. One of these areas was located on floor 81 and floor 82 at the northeast corner of the tower. Flames were evident from windows on either side of the corner as well as the corner itself, which had become exposed by removal of the corner facing during the plane strike. This area is in the vicinity of large piles of debris formed during the plane strike. The second fire was located primarily on floor 79 just to the left of the center (roughly from windows 79-231 to 79-238) of the north face. This is in the area of the second debris pile described earlier. Both of these fires died slowly with time when compared to fires at other locations in WTC 1 and WTC 2. Both were still burning lightly when the tower fell 56 minutes after the plane strike.

A curious aspect of the fire behavior is the existence of an area of the building façade between these two fire locations on the north face where very iittle fire and/or smoke was observed before the tower collapsed. This area is roughly rectangular in shape, covering floor 80 to floor 82 and extending across windows 249 to 239 . Infrared images recorded shortly following the plane strike showed that this region was quite cool relative to other sections close to the fires. This area will be referred to as the "cold spot." Spreading fires seemed to move around this cold spot. 
In general, the fires in WTC 2 appeared to be less active than those observed in WTC 1. The fires covered a smaller area of the façade and did not spread as quickly. This is true even when the shorter time between the plane strike and collapse for WTC 2 ( 1 hour 42 minutes for WTC 1 and 56 minutes for WTC 2) is taken into account. Nevertheless, there was significant fire spread, and instances of rapid fire growth similar to those seen in WTC 1 did take place.

Around 9:29 a.m., large flames and heavy smoke erupted from an area on the north face just to the right of the cold spot (around window 83-236) on floor 83. Four minutes and forty-one seconds later, flames suddenly appeared at a separate location on the same floor further to the right near window 83-226. Another area of fire formed just to the right of the cold spot on floor 82 around 9:54 a.m. or 5 minutes before the collapse. The fires on floor 79 of the north face also spread towards the west, approaching the western edge of the tower just prior to the collapse.

Initial fire growth on the east face was on floor 82. Around 9:12 a.m., flames could be seen in nearly half of the windows on this floor, and heavy smoke was pouring from additional windows. Only limited fire was evident on lower floors at this time. The fires on floor 82 grew smaller after this time, and most were no longer visible when the tower collapsed. Around 9:35 a.m., heavy flames and smoke appeared over large areas of floor 79 and floor 80 . These fires abruptly died down 45 seconds later, before growing back slowly during the remainder of the time before the tower collapsed.

In the early period following the plane strike, fire growth on the south face was seen primarily on floor 81 with active fires present on both sides of the airplane strike location. Smaller isolated fires were present on other floors around the area damaged by the airplane. These fires were relatively quiet and stationary until just prior to the collapse. At 9:56 a.m., there was a sudden release of smoke along much of floor 80 extending from the area of the plane strike to near the western edge. During the next 2 minutes, an intense fire developed covering approximately windows 81-441 to 81-454.

No smoke or fire was observed near the floors struck by the airplane on the west face of WTC 2 . Some smoke was apparent at windows higher on the face. This was most likely coming from windows broken by occupants located on these floors.

\section{H.7 EVIDENCE FOR COLLAPSED FLOORS IN WTC 2}

\section{H.7.1 Hanging Objects}

In the May 2003 Investigation Progress Report (NIST 2003), a photograph was shown in which there appeared to be a floor draped across a number of windows extending roughly from 310 to 342 across the east face of floor 82 of WTC 2. Figure H-22 compares an image taken shortly after the plane strike at 9:03 a.m. and one taken at 9:55 a.m. shortly before the tower collapsed. At the earlier time, the hanging object is already present, but is seen through the windows draped much higher on the floor 82 . An interpretation consistent with these observations is that floor 83 along the east side of WTC 2 was partially collapsed over a significant fraction of its length by the passage of the plane through the building. At the later time the floor has sagged further. By reviewing a number of photographs and videos, it has been determined that the change in floor position occurred between 9:34 a.m. and 9:38 a.m. 


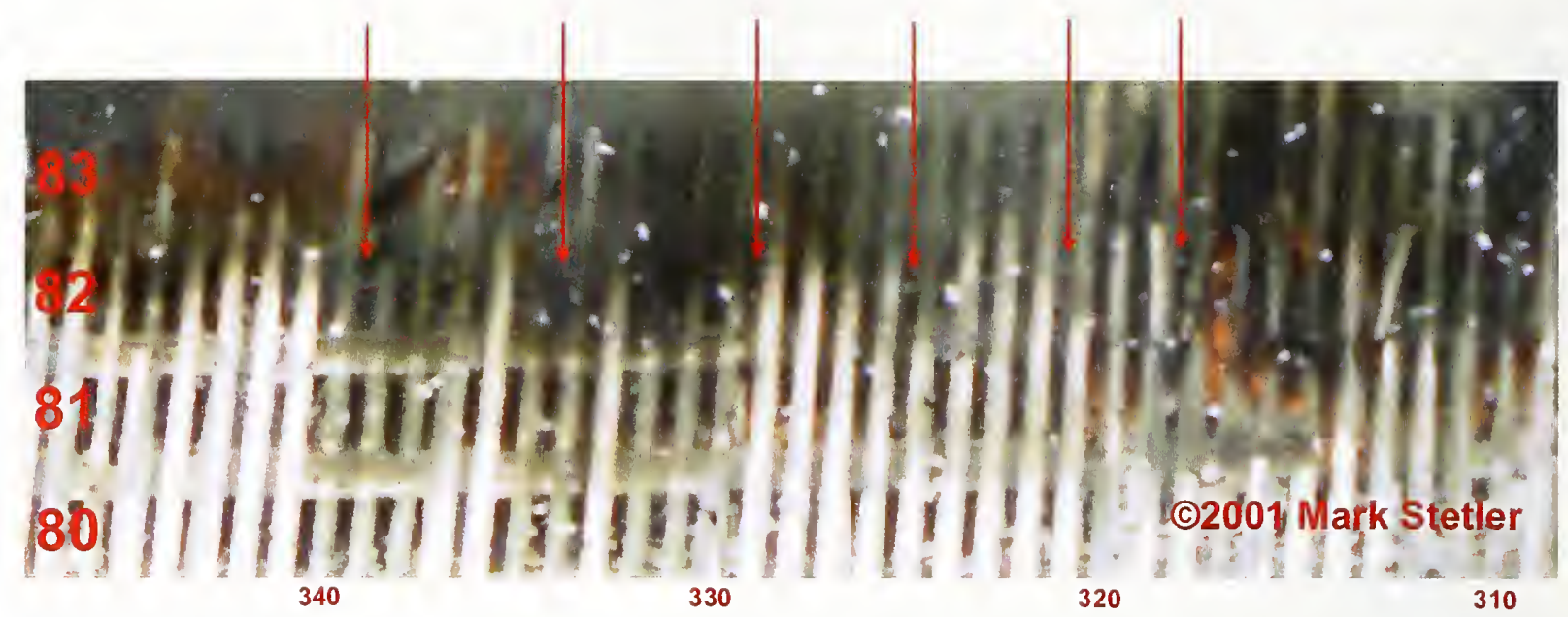

9:03:41 a.m.

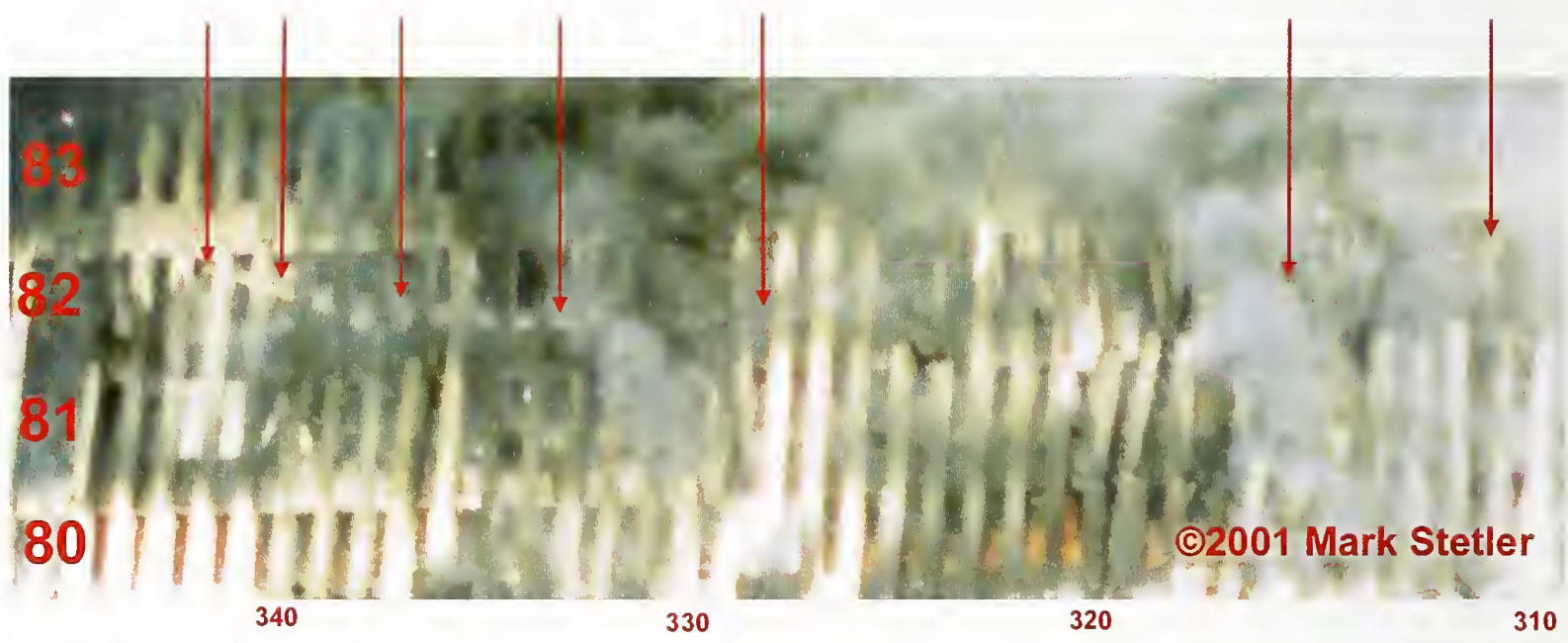

9:55:04 a.m.

Figure H-22. Images of the east face of WTC 2 taken shortly after the plane struck and shortly before the tower collapse are shown. An object that is most likely a floor can be seen through windows on floor 82 as indicated by the arrows. The object has dropped lower between the times the two photographs were taken.

Very similar objects, albeit of shorter length, are seen hanging in windows in images taken from the north. These objects are apparent in Fig. $\mathrm{H}-21$ hanging below floors 81, 82, and 83. As seen through windows on floor 82 (corresponding to the floor 83 ), the floor appears to have split into at least two sections.

\section{H.7.2 Molten Material}

It has been reported in the FEMA report (McAllister 2002) as well as in the media that what appeared to be molten metal was observed pouring from the north face near the northeast corner. This is the area where the sustained fires were seen. Video records and photographs indicate that the material first 
appeared at 9:51:52 a.m. and continued to pour intermittently from the building until the time of collapse. Some of the material can be seen falling in Fig. H-21. Close-up video and photographs of the area where the material is pouring from have been examined and show that it is falling from near the top of window $80-256$. The most likely explanation for this observation is that the material had originally pooled on the floor above, that is, floor 81 , and that it was allowed to pour out of the building when this floor either pulled away from the outer spandrel or sank down to the point where the window was exposed. The fact that the material appears intermittently over a several minute period suggests that the floor was giving way bit by bit.

The composition of the flowing material can only be the subject of speculation, but its behavior is consistent with it being molten aluminum. Visual evidence suggests that significant wreckage from the plane passed through the building and came to rest in the northeast corner of the tower in the vicinity of the location where the material is observed. Much of the structure of the Boeing 767 is formed from two aluminum alloys that have been identified as 2024 and 7075 and closely related alloys. These alloys do not melt at a single temperature, but melt over a temperature range from the lower end of the range to the upper as the fraction of liquid increases. The Aluminum Association handbook (Aluminum Association 2003 ) lists the melting point ranges for the alloys as roughly $500{ }^{\circ} \mathrm{C}$ to $638^{\circ} \mathrm{C}$ and $475^{\circ} \mathrm{C}$ to $635^{\circ} \mathrm{C}$ for alloys 2024 and 7075, respectively. These temperatures are well below those characteristic of fully developed fires (ca. $1,000{ }^{\circ} \mathrm{C}$ ), and any aluminum present is likely to be at least partially melted by the intense fires in the area.

\section{H.8 PROGRESS ON COLLECTION OF IMAGES AND ANALYSIS FOR WTC 7}

Visual material is also required to characterize the initial damage to, fire spread in, and collapse behavior of WTC 7. Considerable useful material has been collected, but the visual record for times between the collapses of WTC 1 and WTC 7 is much less complete than those for the two towers. The reasons for this are easy to understand. Following the collapses of the towers, most people were focused on escape or rescue. A large dust cloud was formed by the collapses, and fires developed that generated large amounts of smoke. Both tended to obscure views of WTC 7, particularly from the south due to the northwesterly wind direction on September 11, 2001.

Both photographs and videos have been included in the database that show fires and damage to the east, north, and west faces of WTC 7. Some of this material has been timed, but in general the record is insufficient to allow generation of a complete time line of fire behavior for the relevant period. Numerous images show the upper portion of WTC 7 from the south, but the actual face of the building is generally obscured by smoke. No clear images of the lower portion of the south face have been obtained despite a careful search and repeated appeals for the public's help. This is particularly unfortunate since most of the damage caused by the collapses of the towers, and particularly WTC 1, should have occurred on this face.

There is considerable interest in images showing the collapse of WTC 7. Currently, there are at least four videos in the database that include the collapse, primarily from northerly directions, as well as several photographs. While not ideal, these are providing adequate information for characterizing the collapse sequence, and some progress along these lines has been made. 
An effort has begun to map out the same information concerning fires, smoke, and windows as in the towers using visual material in the database. This effort will continue with a goal of mapping out as much of the fire time line as possible based on the material in the database.

\section{H.9 SUMMARY WITH KEY FINDINGS}

This section provides a brief summary of progress on the collection and analysis of visual data along with key findings.

The approaches used to identify and obtain visual material related to the WTC disaster are described along with the approaches employed by NIST to archive and catalog the material. Material is either saved in its original digital format or digitized and saved, and a commercial software package has been used to provide data entry, a searchable database, and ready access to assets for review. The large numbers of attributes used to characterize the photographs and videos are included.

Separate databases are provided for photographic and video materials. A major effort has focused on assigning accurate times to the material, and the approaches used are summarized. In excess of 6,700 photographs and 6,900 video clips have been included in the databases and 45 percent and 39 percent, respectively, of these have assigned times accurate to 3 seconds or better.

Major events timed to an accuracy of 1 second are:

- First plane strike on WTC 1: 8:46:30 a.m.

- Second plane strike on WTC 2: 9:02:59 a.m.

- Collapse of WTC 2: 9:58:59 a.m.

- Collapse of WTC 1: 10:28:25 a.m.

- Collapse of WTC 7: 5:20:52 p.m.

An approach has been developed to characterize the observed fire behaviors at the periphery of the buildings on a window-by-window basis by determining whether windows are open or closed and whether smoke and/or fire are observed. If smoke is present, it is characterized as "light" or "heavy", and fires are characterized as "spot" (a small local fire), "fire inside," and "external flaming." The observations are coded in separate electronic spreadsheets for each building, façade, and time.

Two approaches are used to visualize the fire-related parameters. The first is a Web-based application that displays single sides of the towers at a single time. The second is a time-dependent threedimensional representation based on Smokeview (Forney and McGrattan 2003; Forney, Madrzykowski, McGrattan, and Sheppard 2003).

Photographs and videos have been used to characterize several aspects related to the plane strikes on the towers and the distribution of damage on the external faces. For WTC 1, locations where the ends of the wings and vertical stabilizer of the tail section struck the north face and the damage to the steel façade are mapped. The behavior of fireballs generated by the release of fuel as a result of the collision of the 
aircraft with the tower and initial tower damage as reflected in broken windows is used to characterize the distribution of damage to the facades of the tower. In addition, it has been shown that an exterior panel section from the south face was dislodged and landed on the ground. It contained an aircraft wheel that passed through the tower.

The following conclusions are reached concerning the immediate effects of the plane strike on WTC 1:

- The airplane struck columns on the north face ranging from 109 to 152 and covering floor 93 to floor 99 .

- Damage and initial fire growth were greater on the east face of the tower than on the west. Significant damage and early fire growth occurred on the west side of the south face, but not on the east side.

- A three-story panel section was knocked from the south side of the tower and had an aircraft wheel lodged in window 95-329.

Visual evidence related to the plane strike on the south face of WTC 2 is more extensive than for WTC 1. This has allowed additional analyses beyond the mapping of damage on the plane strike face and façade damage to the remaining faces. The following conclusions have been reached concerning the immediate effects of the plane strike on WTC 2:

- The aircraft struck the tower with a measured speed of 545 miles per hour \pm 18 miles per hour.

- The collision of the aircraft caused a measurable sway of the tower that lasted more than 4 minutes. The period of oscillation was 11.3 seconds.

- The airplane struck columns on the south face ranging from 404 to 443 and covering floor 77 to floor 85 .

- Large areas of the façade were removed and/or damaged along the east face of the tower and on the eastern side of the north face. No façade damage or window breakage was evident on the west face.

- Debris piles are observed in the northeast corner of the tower primarily on floors 80 and 81 . Debris is also evident towards the center of the north face on the floor 79 .

- Column 253 on the north face is broken on the floor 81 and the column 254 is severely distorted.

Detailed maps for fire behavior are currently being made. This update characterizes general fire behaviors for the two towers and notes some particularly interesting observations. For WTC 1, the following observations are highlighted.

- Extensive fires observed immediately following the plane strikes and which are most likely associated with released jet fuel damped down after roughly 60 seconds. 
- In the period following the plane strikes fires tended to reappear over a period of many minutes. Initial fire growth was principally observed on floor 96 and floor 97 on the north face, floor 94 and floor 97 on the east face, floor 96 on the south face, and floor 97 on the west face.

- Observed fire spread rates were quite variable. Examples of both relatively slow and very rapid apparent fire spread are described.

- Interior walls at several locations were inferred to protect areas of the towers for a period of many minutes, though they were typically eventually breached by nearby fires.

- Following the collapse of WTC 2, a large fire appeared and grew rapidly on the west face at floor 104 .

- There was an extensive area of the façade on the eastern side of the south face for which no fire was observed until at least 1 hour following the plane strike. When fires finally did appear in this area their growth was rapid over multiple floors.

- A large amount of smoke was suddenly released from floor 92 on the north face at 10:18:40 a.m. Smoke was expelled simultaneously from other floors and faces. Immediately after the smoke release rapid fire growth was observed at an isolated location on floor 95 and across much of the west side of the north face on floor 92 .

Observed fire behaviors for WTC 2 were somewhat different than for WTC 1 . This is true even when the differences in times between plane strikes and collapses ( 1 hour and 42 minutes for WTC 1 and 56 minutes for WTC 2) are considered. The following observations concerning fire behavior in WTC 2 are emphasized:

- Extensive fires observed immediately following the plane strikes and most likely associated with released jet fuel damped down after roughly 60 seconds.

- Two regions of intense fire remained following the initial fire period due to jet fuel burning. These fires were located on floor 81 and floor 82 in the northeast corner and towards the center of the north face on floor 79. These fires burned for longer periods than observed elsewhere in WTC 1 and WTC 2. They are located in regions of the tower where debris piles are observed.

- No large fires were observed in a multi-floor region on the north face located between the two fire areas described in the last bullet.

- Initial fire growth in areas away from the sustained fires was along the east face of floor 82 . Large fires did not appear on lower floors of this face until later and were sporadic in space and time.

- Prior to the tower collapse, fire spread primarily from east to west was observed on floors 79 , 82 , and 83 of the north face. 
- A sudden release of smoke from windows on the west side of the south face on floor 80 occurred at 9:56:37 a.m. This was followed very shortly by the appearance of heavy fire.

A number of photographs and videos show what appears to be floor 83 hanging across window openings over a large fraction of floor 82 on the east face of WTC 2 . This object is observed very shortly after the plane strike and is found to drop lower prior to the tower collapse. On the north face, shorter lengths of what appear to be floors 81,82 , and 83 are seen hanging through the windows below.

Starting around 9:52 a.m., a molten material began to pour from the top of window 80-256 on the north face of WTC 2. The material appears intermittently until the tower collapses at 9:58:59 a.m. The observation of piles of debris in this area combined with the melting point behaviors of the primary aluminum alloys used in the Boeing 767 suggest that the material is molten aluminum derived from aircraft debris located on floor 81 .

The visual record for the period following the collapses of the two towers is much less complete than prior to this time. In addition to the general chaos caused by the collapses, significant dust and smoke from fires started by the collapses obscured the site. As a result, it has not been possible to identify clear visual images showing the damage to the south face of WTC 7 caused by the collapses of WTC 1 and WTC 2. The number of videos and photographs showing fires on the east, north, and west faces of WTC 7 is limited and sporadic. The images that are available are being used to generate an approximate time line for fire growth and spread.

\section{H.10 REFERENCES}

Forney, G. P., and K. B. McGrattan. 2003. User's Guide for Smokeview Version 3.1: A Tool for Visualizing Fire Dynamics Simulation Data. NISTIR 6980. National Institute of Standards and Technology. Gaithersburg, MD.

Forney, G. P., D. Madrzykowski, K. B. McGrattan, and L. Sheppard. 2003. Understanding fire and smoke flow through modeling and visualization, IEEE Computer Graphics and Applications, vol. 23, p. 6-13.

McAllister, T., ed. 2002. World Trade Center Building Perfornance Study: Data Collection, Preliminary Observations, and Recommendations. FEMA 403. Federal Emergency Management Agency.

Washington, DC, May.

NIST (National Institute of Standards and Technology). 2003. May 2003 Progress Report on the Federal Building and Fire Safety Investigation of the World Trade Center Disaster. NIST Special Publication 1000-3. U.S. Department of Commerce, Washington, DC, May.

The Aluminum Association. 2003. Aluminum Standards and Data - 2003. The Aluminum Association, Inc., Washington, DC. 
This page intentionally left blank.

H- -44 


\section{TABLE OF CONTENTS}

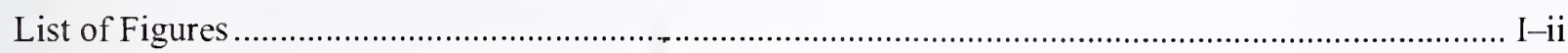

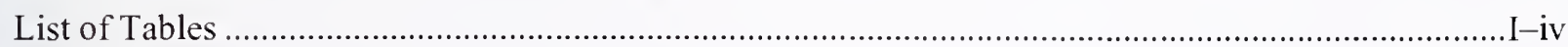

\section{Appendix I}

Interim Report on Assessment of Sprayed Fireproofing in the WTC Towers-

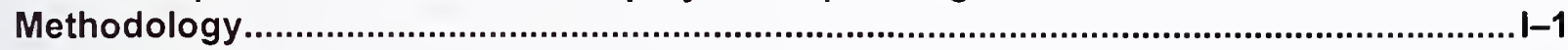

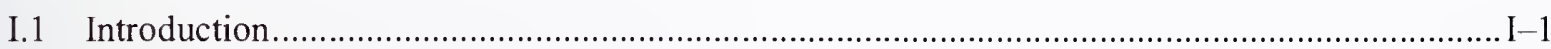

I.2 Sensitivity of Thermal Response to Fireproofing Geometery ................................................. I-1

I.3 In-Place Conditions of Fireproofing before Impact ................................................................... I-7

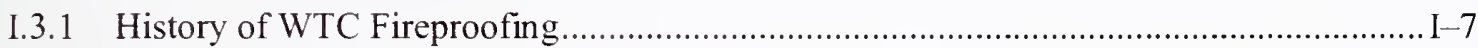

I.3.2 Specified Thickness of Fireproofing ................................................................................ $\mathrm{I}-8$

I.3.3 As-Applied Thickness and Variability .............................................................................

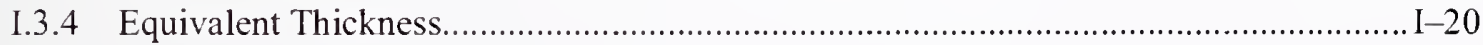

I.3.5 Thickness of SFRM for Use in Analyses ...................................................................

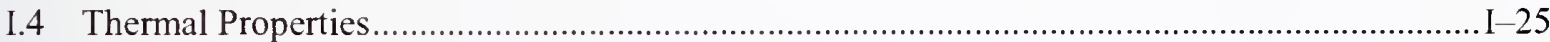

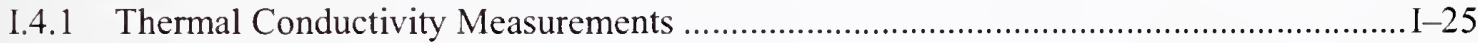

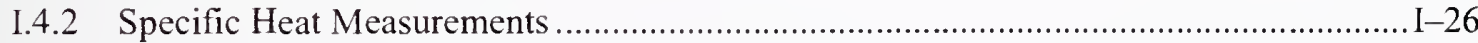

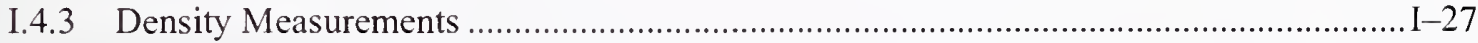

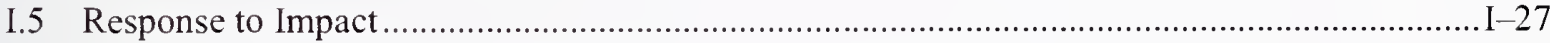

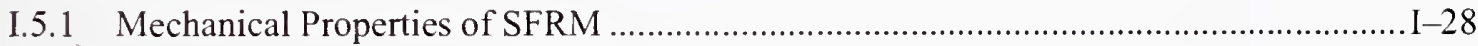

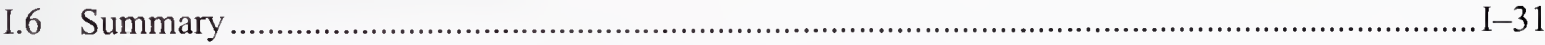




\section{LIST OF FIGURES}

Figure I-1. Model used to study effects of fireproofing thickness and variability of thickness on steel temperature

Figure I-2. Temperature distribution after $1 \mathrm{~h}$ of exposure to gas temperature of $1,100{ }^{\circ} \mathrm{C}$ $(1,373 \mathrm{~K})$.

Figure I-3. Variation of steel temperature (at a point 6 in. from end of plate) with time for different average thicknesses of fireproofing I-4

Figure I-4. Example of "gap" in fireproofing on diagonal member of a bridging floor truss................I-5

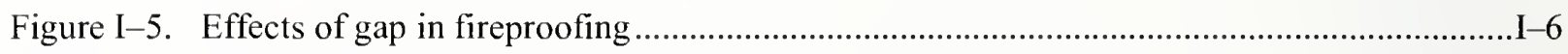

Figure I-6. Example of plot matrix from sensitivity study of the effects of missing fireproofing and variability of fireproofing thickness on steel temperature

Figure I-7. Dotplot of average thickness from floor trusses for floors 23 and 24, histogram of average thickness, normal probability plot of average thickness, histogram of natural logarithm of average thickness, and probability plot of natural logarithm of average thickness.

Figure I-8. Example of measurement procedure used to estimate fireproofing thickness from photographs.

Figure I-9. Normal probability plot of estimated fireproofing thickness based on photographs, and normal probability plot of natural logarithms of thickness.

Figure I-10. Dotplot of individual thickness measurements on floor trusses from Port Authority Construction Audit Reports, histogram of thickness measurements, normal probability plot of thickness measurements, histogram of natural logarithms of thickness measurements, and normal probability plot of natural logarithm of thickness measurements. I-18

Figure I-11. Fireproofing thickness on floor trusses in upgraded portions of WTC towers I-19

Figure I-12. Individual and average thickness for core columns, normal probability plot of individual measurements on columns, individual and average thickness for core beams, and normal probability plot of individual measurements on beams.

Figure I-13. Randomly generated thickness profiles with average thickness of 0.75 in. and standard deviation of 0.3 in., cumulative element size, and deformation of $1 \mathrm{in}$. bar compared with deformation for uniform thickness of fireproofing.

Figure I-14. Randomly generated thickness profiles with average thickness of 2.5 in. and standard deviation of 0.6 in., normal probability plots of thickness values, and deformation of $1 \mathrm{in}$. bar compared with deformation for uniform thickness of fireproofing.

Figure I-15. Preliminary test results: thermal conductivity as a function of temperature, and specific heat as a function of temperature.

Figure I-16. Pull-off test of SFRM applied to steel plate. 
Figure I-17. Derivation of acceleration to dislodge SFRM from planar substrate................................ I-29

Figure I-18. Derivation of acceleration to dislodge SFRM surrounding a round bar. .......................... I-30 


\section{LIST OF TABLES}

Table I-1. Specified passive fire protection.

I-9

Table I-2. Average fireproofing thickness from six measurements taken in 1994 on each of 16 random floor trusses on floors 23 and 24 of WTC 1 $.1-11$ 


\section{Appendix I \\ INTERIM REPORT ON ASSESSMENT OF SPRAYED FIREPROOFING IN THE WTC TOWERS-METHODOLOGY}

\section{I.1 INTRODUCTION}

The structural steel in the World Trade Center (WTC) towers was "fireproofed" with sprayed fire resistive materials (SFRMs). These materials are packaged as dry ingredients, and water is added by a pressurized system as the materials are sprayed onto the steel. The water mixes with the cementitious materials and provides "stickiness" that allows the SFRM to adhere weakly to the steel. With time, the cementitious materials harden, and excess water evaporates. When dry, SFRMs provide an insulation barrier to reduce the vulnerability of the steel to excessive temperature rise during a fire.

Analysis of the effects of the fires on the structural capacity of the damaged WTC towers as a function of time requires knowledge about the condition of fireproofing on the various structural components, namely, the exterior columns, the spandrel beams, the floor trusses, and the core columns. Because of the method of application, sprayed fireproofing will have variable thickness, especially when applied to long, thin elements such as the diagonals and chords of the floor trusses. In addition, fireproofing was dislodged during the impact, either from direct impact by debris or from vibrations of the members. The thermal properties of the fireproofing also need to be known as a function of temperature.

The thermal-structural analysis of the WTC towers focused on two objectives: (1) analysis of the undamaged buildings exposed to postulated fires, and (2) analysis of damaged buildings exposed to the fires that occurred after impact. In order to reduce the uncertainties in the calculated thermal histories of various structural elements, the condition of the sprayed fireproofing as it existed on September 11, 2001, needs to be estimated as accurately as possible. In addition, reasonable estimates of the extent of fireproofing dislodged by the aircraft are needed. This appendix discusses the approach that will be used for this purpose.

To gain an understanding of the effect of fireproofing thickness and its variability on the steel temperature during exposure to fire, a simple finite-element model was used for a sensitivity study. The information gained from that study is reviewed first. A brief summary of the construction history of the sprayed fireproofing in WTC 1 and WTC 2 is presented. This is followed by a quantitative assessment of in-place thickness and its variability based on available data. The rationale for the thickness of fireproofing to be used in the structural fire endurance analyses is presented. The tests conducted to determine the thermal properties of fireproofing materials similar to those used in the WTC towers are reviewed. The approach used to gain an understanding of the inherent fragility of sprayed fireproofing is discussed, and the scheme for estimating the extent of damage during impact is summarized.

\section{I.2 SENSITIVITY OF THERMAL RESPONSE TO FIREPROOFING GEOMETERY}

The fireproofing thickness has a great effect on the thermal response of the structural elements for a given fire condition. While others have considered the effect of thickness of fireproofing, the effect of the 
variation of thickness along the length of a member is not well known. A sensitivity study using finite element modeling of heat transfer was conducted to investigate the sensitivity of steel temperature to the variability in fireproofing thickness.

The simplified model that was used is shown in Fig. I-1. A 1 in. thick, 60 in. long steel plate (cyan color) was coated with fireproofing material (purple color) and subjected to the uniform radiative flux arising from a $1,100{ }^{\circ} \mathrm{C}$ fire. As shown in Fig. I-1 (b), the fireproofing is modeled with a layer of finite elements ( $0.125 \mathrm{in}$. thick and $0.6 \mathrm{in}$. long) having the thermal properties of fireproofing (purple). A parametric study was conducted with average thickness of fireproofing varying from 0 in. to 2 in. in increments of $1 / 4$ in. The effect of variability in thickness was modeled by imposing a normal probability distribution on the fireproofing thickness along the length of the steel plate. The assumed standard deviation varied from 0 in. (uniform thickness) to 1 in. A psuedo-random number generator was employed to determine the thickness at each cross section based on the assumed average thickness and standard deviation. The layer representing fireproofing was taken to be twice the average thickness, and the thickness of fireproofing at any cross section was modeled by assigning a low heat capacity and a high thermal conductivity to those elements that do not provide fireproofing. Figure I-1 (c) shows an example of variable thickness fireproofing; in this case, the average thickness is $1 \mathrm{in}$. and the standard deviation is $3 / 8$ in.

When the model in Fig. I-1 is exposed to the thermal flux representing an $1,100^{\circ} \mathrm{C}$ fire, the surface of the insulation heats up quickly to the gas temperature $(1,100+273=1,373 \mathrm{~K})$. Numerical simulation was performed over a $2-\mathrm{h}$ period, and the steel temperature at five locations was recorded at $30 \mathrm{~min}, 60 \mathrm{~min}$, $90 \mathrm{~min}$, and $120 \mathrm{~min}$ of exposure. The temperature recording locations are $6 \mathrm{in}$. from each end and at 12 in. intervals, which are shown as numbers 1 to 5 in Fig. I-1 (a). The initial temperature of the model is $300 \mathrm{~K}$.

Figure $\mathrm{I}-2$ shows temperature contours (in K) through the fireproofing and steel at $60 \mathrm{~min}$ after initial exposure for the model shown in Fig. I-1 (a). The fireproofing surface temperature is close to the gas temperature of $1,373 \mathrm{~K}$, while the steel temperature is $311 \mathrm{~K}$. If the fireproofing were of uniform thickness, the isotherms would be a series of lines parallel to the plate. It is seen that, when the thickness of fireproofing is variable, the isotherms follow the shape of the fireproofing surface contour. Thus, the temperature history at any point in the steel depends on the loc al thickness of the fireproofing.

Figure $\mathrm{I}-3$ shows the steel temperature at the far sensor \#1 (6 in. from the end) as a function of time for various insulation thicknesses ranging from 0 in. to 2 in. (the thickness is indicated by the numbers on the curves). For the case in Fig. I-3 (a), the fireproofing is of uniform thickness, and for the cases in Fig. I-3 (b), the thickness varies with a standard deviation of $1 \mathrm{in}$. The time to reach a temperature of $600{ }^{\circ} \mathrm{C}$ is used as a measure of relative performance. It is seen that the presence of high variability in thickness has a detrimental effect of the protection provided by the fireproofing. For example, for a uniform thickness of $0.5 \mathrm{in}$., it takes about $60 \mathrm{~min}$ for the steel at point $\# 1$ to reach $600{ }^{\circ} \mathrm{C}$; but when the standard deviation of the thickness is 1 in., the average thickness has to be 1.75 for the same level of thermal protection. 


\section{(a)} 1 in. thick steel plate

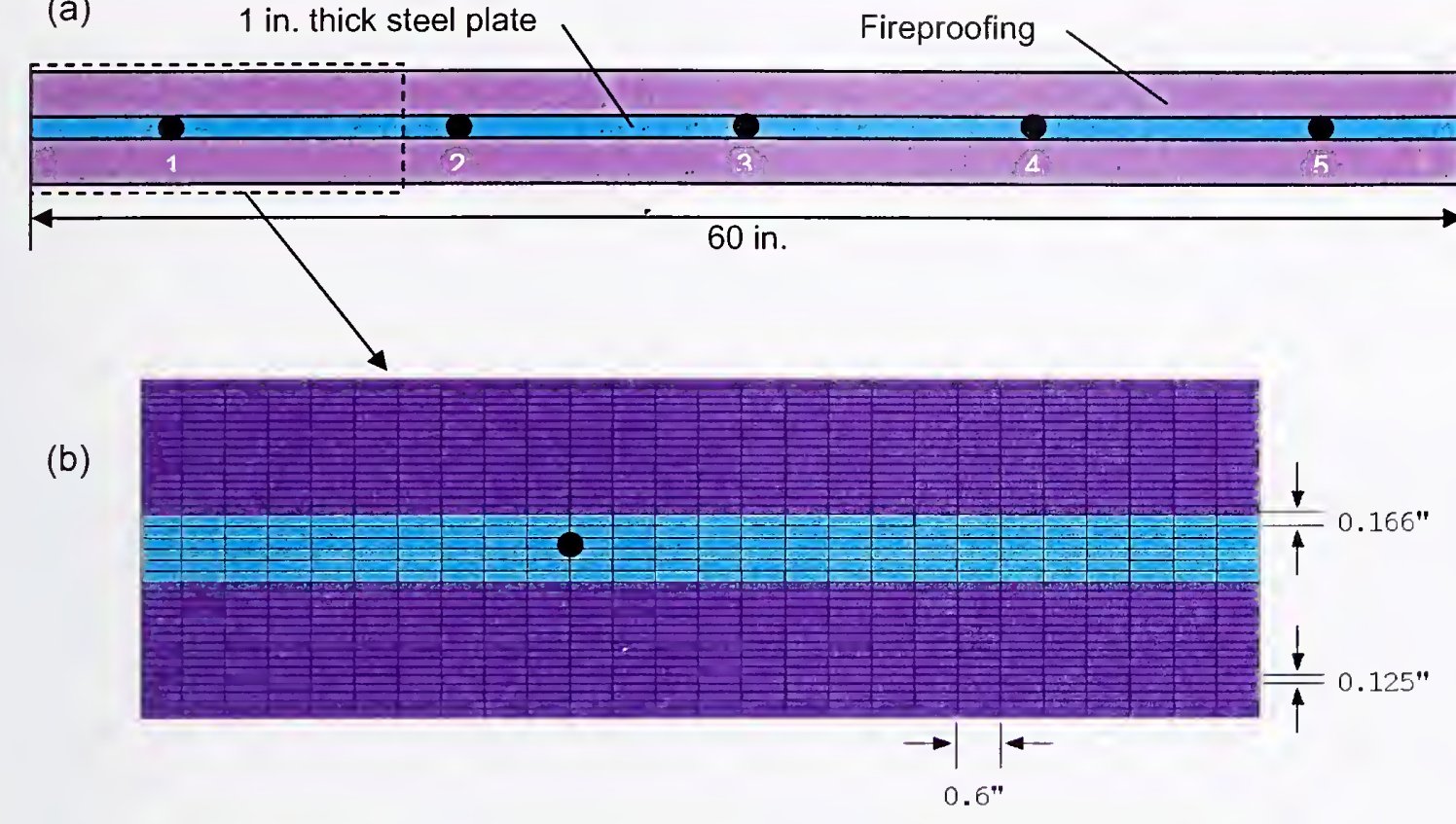

(c)

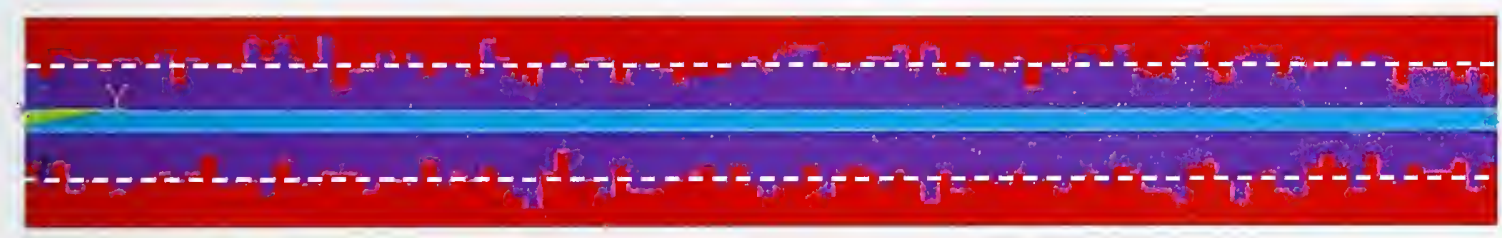

Figure I-1. Model used to study effects of fireproofing thickness and variability of thickness on steel temperature: (a) physical model used in analyses (points 1 to 5 are locations where temperatures are monitored), (b) finite element mesh used to represent physical model, and (c) finite element model to represent variable thicknessof fireproofing (purple) (the elements in red represent material of high thermal conductivity).

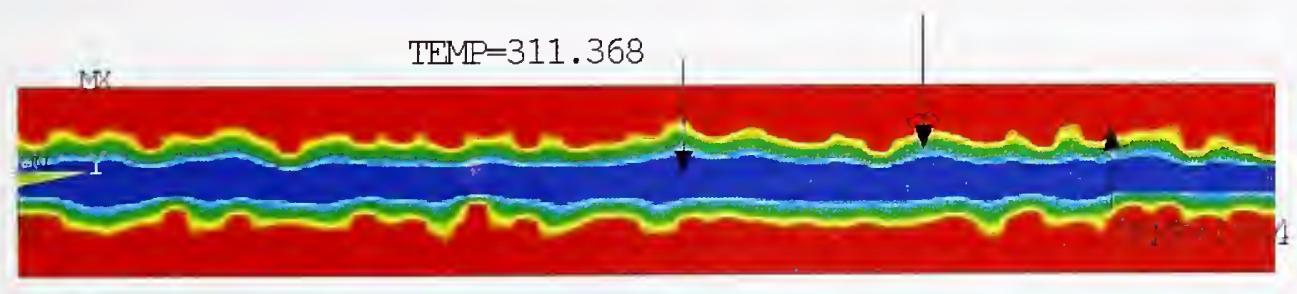

$$
\begin{array}{lllll}
700 & 700 & 900 & 1100 & 1300
\end{array}
$$

Figure I-2. Temperature distribution after $1 \mathrm{~h}$ of exposure to gas temperature of

$$
1,100^{\circ} \mathrm{C}(1,373 \mathrm{~K}) \text {. }
$$



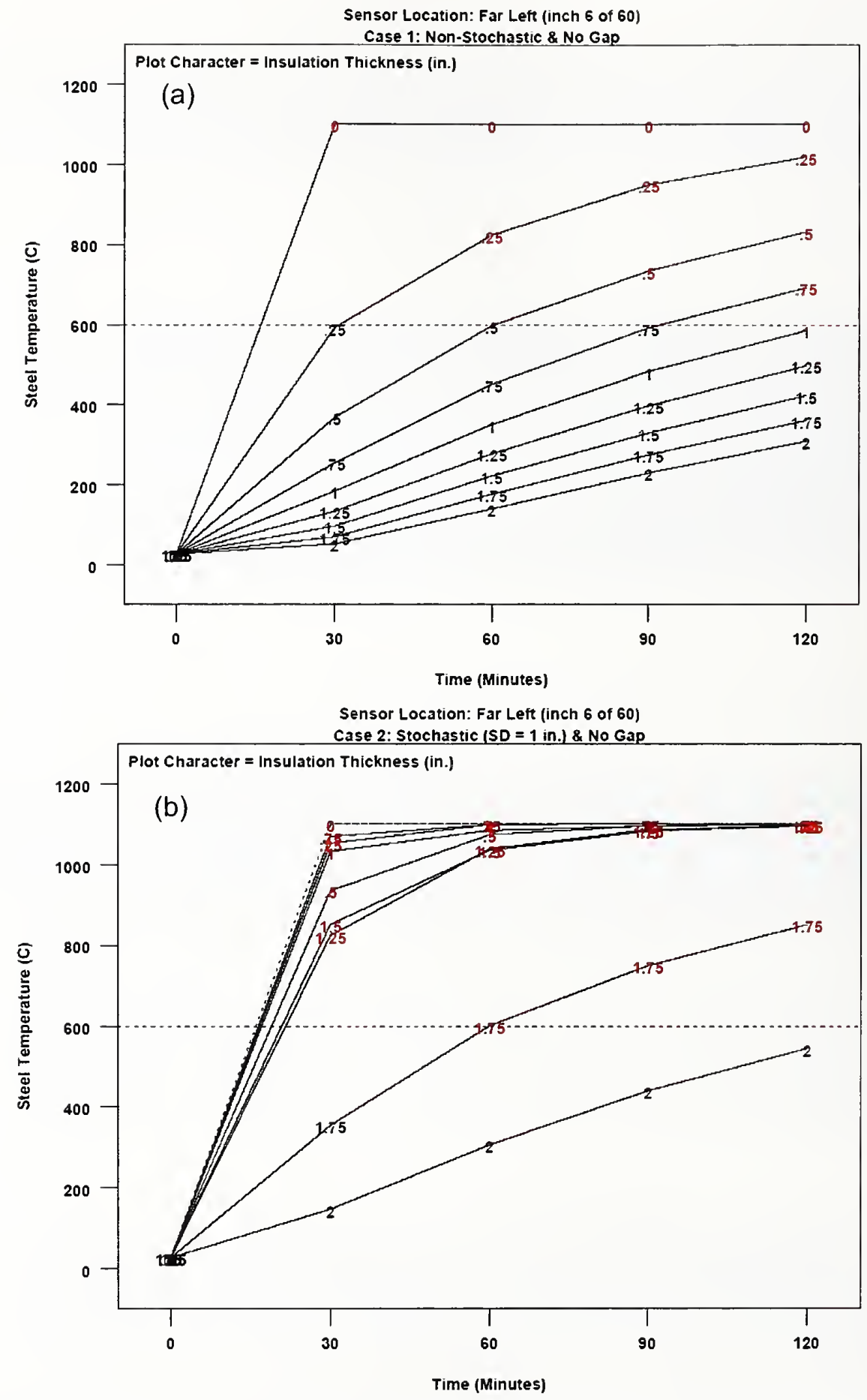

Figure I-3. Variation of steel temperature (at a point 6 in. from end of plate) with time for different average thicknesses of fireproofing (shown as numbers on the curves): (a) uniform thickness, and (b) variable thickness with a standard deviation of 1 in. 
In addition to the effect of variation in thickness, it is important to understand the effect of missing fireproofing over a portion of a member. As an example, Fig. I-4 shows missing fireproofing from a diagonal of a bridging truss of the WTC towers floor system. Figure I-5 (a) shows an example of a numerical model with missing fireproofing. In this case, there is 12 in. of missing fireproofing on the steel plate, which is otherwise protected by 2 in. of uniform thickness fireproofing. Figure I-5 (b) shows the temperature contours (isotherms) at time $50 \mathrm{~min}$. For comparison, Fig. I-5 (c) shows isotherms at the same time in a plate with no gap in the fireproofing. As expected, the bare steel at the missing fireproofing is at the gas temperature, but more importantly the "gap" in fireproofing leads to a "leakage" of heat into the interior steel.

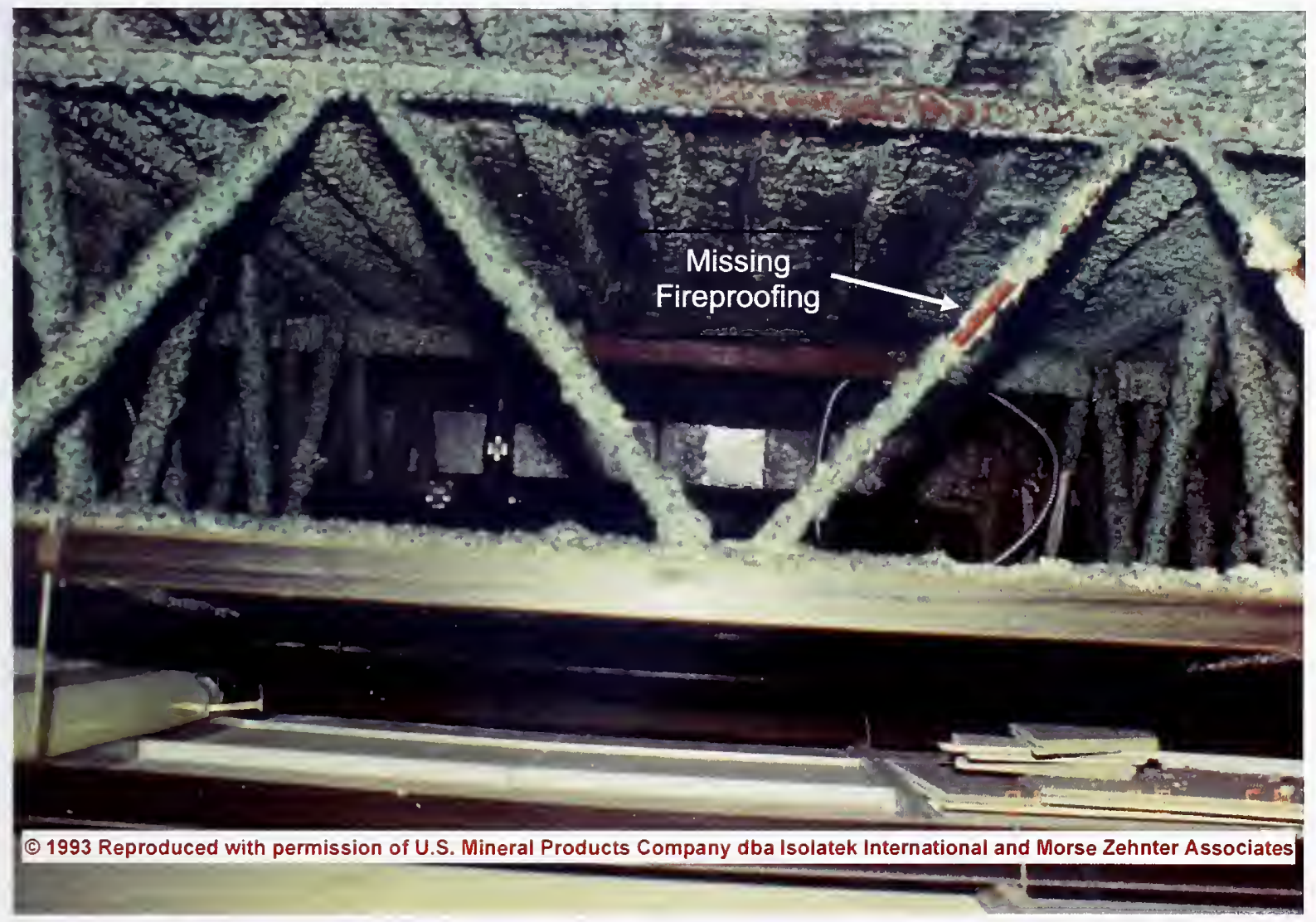

Figure 1-4. Example of "gap" in fireproofing on diagonal member of a bridging floor truss.

The combined effects of variation in thickness of the fireproofing and length of missing fireproofing were examined by a factorial study with the following factors:

- Average thickness of fireproofing varying from 0 in. to $2.0 \mathrm{in.} \mathrm{in} 1 / 4 \mathrm{in}$. increments;

- Standard deviation of fireproofing thickness of 0 in., 0.25 in., $0.5 \mathrm{in.}, 0.75$ in. and $1.0 \mathrm{in.}$; and

- Length of missing fireproofing varying from 0 in. to 30 in., in 6 in. increments. 


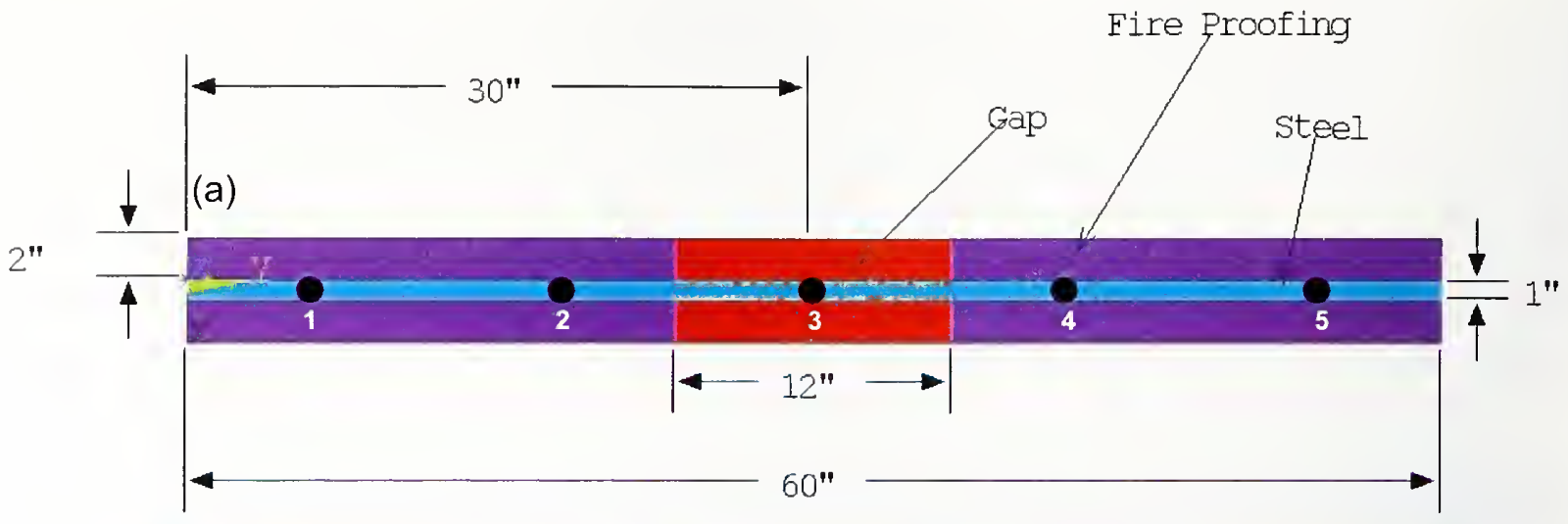

(b)

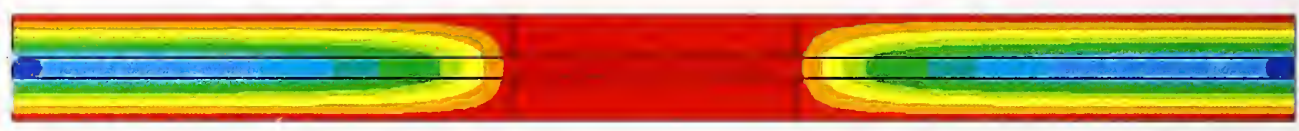

(c)
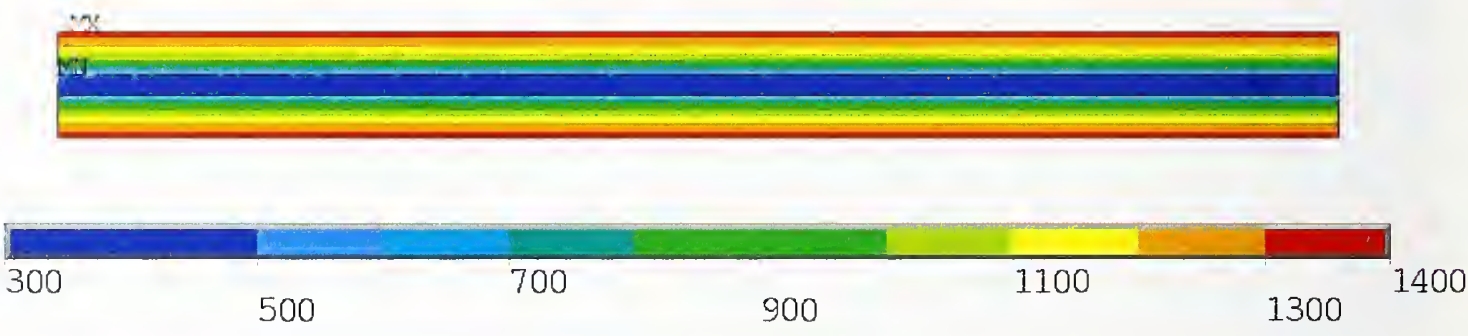

Figure 1-5. Effects of gap in fireproofing: (a) model of plate with fireproofing having 2 in. uniform thickness and 12 in. gap, (b) isotherms $(\mathrm{K})$ at time $=50 \mathrm{~min}$ with $12 \mathrm{in}$. gap, and (c) isotherms without gap.

The results of the sensitivity study can be summarized in a series of plot matrices, which show the time histories of the steel temperature for different combinations of gap length and variability in fireproofing thickness. For example, Fig. I-6 shows the plot matrix for the temperature history at point \#2 (18 in. from the end of the plate). Each plot contains a series of curves representing different average thickness of fireproofing, as in Fig. I-3. Each column of plots represents a constant value of thickness variability (standard deviation), and each row represents a constant gap length. The plot in the upper left corner represents the case of uniform thickness of fireproofing and no gap, which is the same plot as in Fig. I-3(a). (Note that for the case of uniform thickness and no gap, the steel temperature at any point in a cross section is the same along the length of the plate, as shown in Fig. I-5(c).) For gaps of 24 in. and $30 \mathrm{in}$., the temperature at point $\# 2$ rises rapidly because there is no fireproofing on the plate at that location. This explains the shapes of the curves in the two lower rows. In going from left to right in one of the top four rows it is seen that as variability of thickness increases, the time histories shift upward, thereby reducing the time to reach $600^{\circ} \mathrm{C}$. This is the same observation as shown in Fig. I-3. Moving from the top to the bottom in any column shows the effects of increasing gap length. The effect of gap length depends, of course, on where the steel temperature is measured. At a point within the portion of steel that is bare, the temperature rises quickly. At points within the steel that are surrounded with fireproofing, the gap provides a path for heat flow, as shown in Fig. I-5 (b). As a result, points in the steel within the vicinity of the missing fireproofing will experience higher temperatures, as indicated by the rising trend of the curves in going downward from the top of a column in Fig. I-6. The National 
Institute of Standards and Technology (NIST) does not have sufficient information to determine the frequency of occurrence of these gaps or their typical locations. Therefore, gaps in fireproofing will not be considered in the thermal modeling.

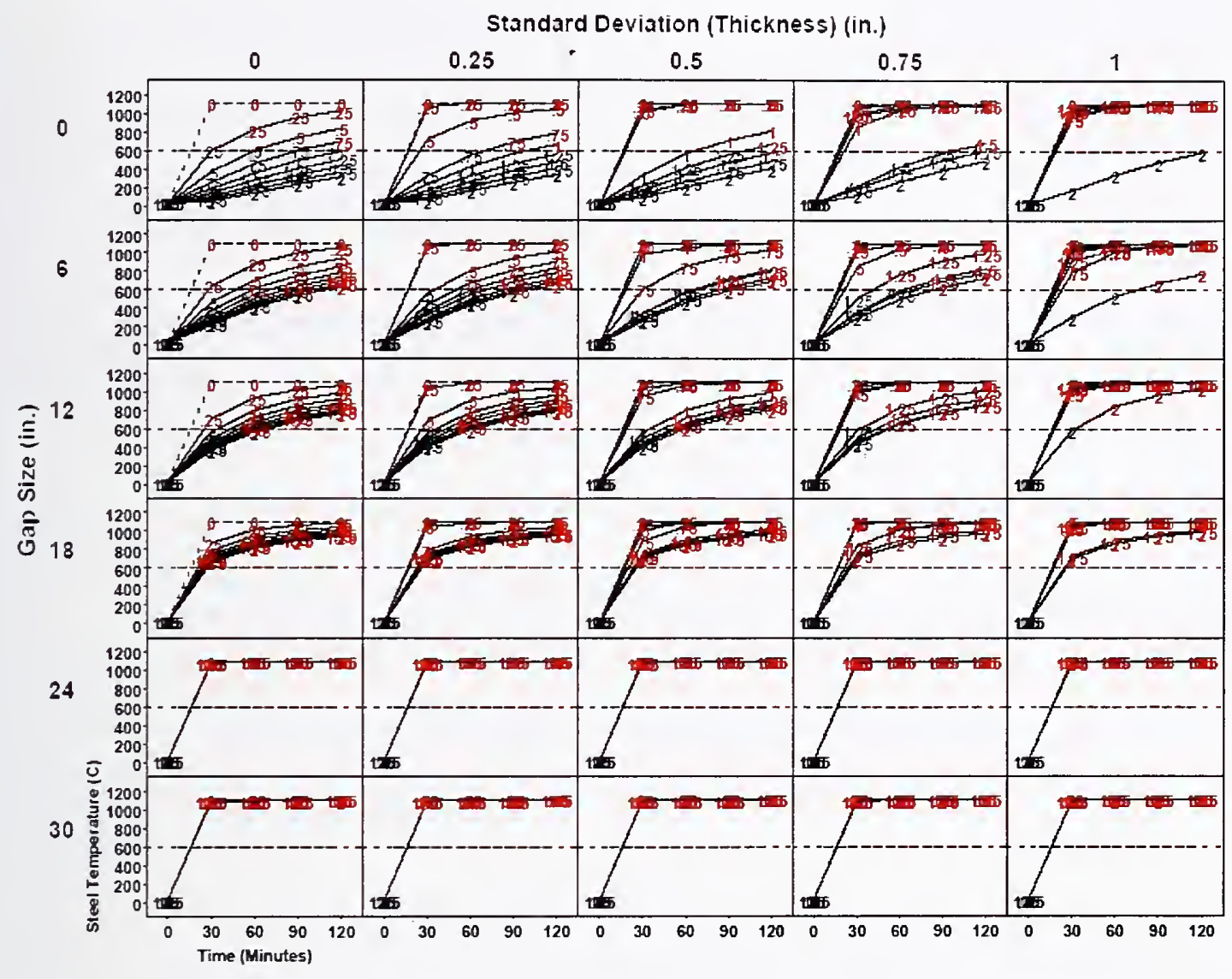

Figure 1-6. Example of plot matrix from sensitivity study of the effects of missing fireproofing and variability of fireproofing thickness on steel temperature. Each graph is a temperature history of the steel at point \#2 (see Fig. I-5) for different thicknesses of fireproofing.

\section{I.3 IN-PLACE CONDITIONS OF FIREPROOFING BEFORE IMPACT}

\subsubsection{History of WTC Fireproofing}

In Appendix 4 of the May 2003 Progress Report (NIST SP 1000-3), the history of the sprayed fireproofing, as reconstructed from available documentation, was reviewed. Basically, the following significant activities took place:

- 1969: Decision made to use 1/2 in. of CAFCO BLAZE-SHIELD Type D (asbestos fibers) sprayed fireproofing. 
- 1970: Use of CAFCO BLAZE-SHIELD Type D was discontinued at the 38th floor of WTC 1; remaining fireproofing to use CAFCO BLAZE-SHIELD Type DC/F (mineral wool fibers).

- 1994: Thickness measurements of fireproofing on trusses of floors 23 and 24 of WTC 1.

- 1995: Port Authority performed study to establish sprayed fireproofing thickness for tenant alterations.

- 1999: Port Authority established guidelines for fireproofing repairs, replacement, and upgrades.

- Late 1990s: Floor truss fire protection upgraded to $11 / 2$ in. during tenant alterations using CAFCO BLAZE-SHIELD Type II. In-place measurements of thickness, density, and bond strength recorded.

\section{I.3.2 Specified Thickness of Fireproofing}

WTC project specifications for spray-applied fireproofing do not provide required material thickness or hourly ratings. However, a letter dated October 30, 1969, from Robert J. Linn (manager, Project Planning, WTC) to Mr. Louis DiBono (Mario \& DiBono Plastering Co., Inc.) states, in part:

...Tower " $A$ " columns that are less than $14 \mathrm{WF} 228$ will require $23 / 16$ " thick of 'Cafco [B]laze-Shield Type D' spray-on Fireproofing. All Tower columns equal to or greater than 14 WF228 will require $13 / 16^{\prime \prime}$ of fireproofing...

All Tower beams, spandrels and bar joists requiring spray-on fireproofing are to have a $1 / 2$ " covering of "Cafco."

No reference is made in this letter to the required thickness of fireproofing of core box columns or exterior built-up columns.

Alcoa was the supplier of the aluminum cladding on the exterior columns (Contract WTC 400.00), and the following "Note 11" was included among the "General Notes" of their drawings:

11. Exterior column and spandrel fireproofing-CAFCO BLAZE SHIELD Type D Fireproofing. Interior column and spandrel fireproofing-Vermiculite plaster aggregate fireproofing with finished plaster coat on exposed areas of columns. ( $3 \mathrm{hr}$ on spandrels, $4 \mathrm{hr}$ on columns)

\section{Fireproofing Thickness}

$\begin{array}{llc}\text { Rating } & \text { Cafco } & \text { Vermiculite Aggregate } \\ 4 \mathrm{hr} \text { (heavy column) } & 13 / 16^{\prime \prime} & 7 / 8^{\prime \prime} \\ 3 \mathrm{hr} \text { (spandrels) } & 1 / 2^{\prime \prime} & 1 / 2^{\prime \prime}\end{array}$


In 1995, the Port Authority performed a study to establish the requirements for applying spray-on fireproofing to the floor trusses in the case of new construction (alterations conducted when tenants vacated the space) in the towers. The study estimated the fireproofing requirements for the floor trusses of the towers based on "the fireproofing requirements for Design No. G805 contained in the Fire Resistance Directory" of Underwriters' Laboratories. The study concluded that $11 / 2 \mathrm{in}$. of spray-on mineral fiber fireproofing, "when applied directly to the chords and web members," was sufficient to achieve the required $2 \mathrm{~h}$ rating for the floor trusses. In the years between 1995 and 2001, fireproofing was upgraded in a number of the floors affected by the fires on September 11, 2001.

The specified fire protection is summarized in Table $\mathrm{I}-1$.

Table 1-1. Specified passive fire protection.

\begin{tabular}{|c|c|c|c|c|}
\hline Structural Component & Member Size & Location & Material & Thickness (in.) \\
\hline Floor trusses & All & NA & CAFCO DC/F & $1 / 2$ \\
\hline \multirow[t]{2}{*}{ Interior columns ${ }^{\mathrm{a}}$} & $<14$ WF 228 & $\mathrm{NA}$ & $\mathrm{CAFCO} \mathrm{DC/F}$ & $23 / 16$ \\
\hline & $\geq 14$ WF 228 & NA & CAFCO DC/F & $13 / 16$ \\
\hline \multirow[t]{2}{*}{ Exterior columns } & "heavy" & $\begin{array}{l}\text { Exterior } \\
\text { faces }\end{array}$ & $\mathrm{CAFCO} \mathrm{DC/F}$ & $13 / 16$ \\
\hline & "heavy" & Interior faces & $\begin{array}{c}\text { Vermiculite } \\
\text { aggregate }\end{array}$ & $7 / 8$ \\
\hline \multirow[t]{2}{*}{ Spandrel beams } & All & Exterior face & CAFCO DC/F & $1 / 2$ \\
\hline & All & Interior face & $\begin{array}{l}\text { Vermiculite } \\
\text { aggregate }\end{array}$ & $1 / 2$ \\
\hline
\end{tabular}

a. No thicknesses specified for core beams and box columns.

Key: NA, not applicable.

In a letter dated July 25, 1966, from Emery Roth and Sons to the Port of New York Authority, it is stated "Since the deck is non-structural it will not be fire proofed." Photographs show that in some areas the underside of the metal deck was indeed not fireproofed, while in other areas fireproofing appears to be present but of undetermined thickness and possibly resulting from overspray. Photographs reveal that the dampers and damper saddles were not fireproofed. Additionally, it is unclear whether the bridging trusses were required to be fireproofed in all areas. Subsequent to the design and construction of the WTC towers, some information has been found that further describes the elements of the structural systems that were indeed fireproofed.

\subsubsection{As-Applied Thickness and Variability}

The actual thickness of a spray-applied fire protection material generally exceeds the specified thickness by some amount. Since both towers collapsed on September 11,2001, and most of the fireproofing was either dislodged or abraded (or scraped) off in the collapse, no examples remain of the "as installed" condition of the fireproofing. To make an estimate of the as-applied thickness and variability in thickness, several sources of information have been employed, including measurements taken by the Port Authority, condition surveys and anecdotal information, and photographs and video clips showing the condition of the fireproofing in selected areas. Each of the structural components or systems is considered here separately. 


\section{Steel Truss-Supported Floor System}

Qualitative information on the "as installed" fireproofing thickness for the floor system first appears in Sample Area Data Sheets from 1990, in which comments on the state of the in-place fireproofing were recorded. As an example, the data sheet for floor 29 of WTC 1 states the following for the South West quadrant of the floor:

Fluffy spray-on fireproofing coating the support beams, joists, and deck above the ceiling. The thickness of the material on the beams and joists was consistently about $1 / 2^{\prime \prime}$ Regarding the deck it ranged from very sparce $[\mathrm{sic}]$ in areas to $1 / 4^{\prime \prime}$ other areas.

Similar statements were recorded for the remaining quadrants of the floor.

Information regarding quantitative inspection of existing fireproofing appears in documentation from 1994. That year, the Port Authority performed a series of thickness measurements of the existing fireproofing on floors 23 and 24 of WTC 1. Six measurements were taken from "both flanges and web" of each of 16 randomly chosen trusses on each floor at those locations where the fireproofing was not damaged or absent.

The averages of six measurements per joist that were recorded on the two floors are presented in Table I-2. Measured average thickness varied between 0.52 in. and 1.17 in. For the 32 measurements (16 on each floor), the overall average was 0.74 in. and the standard deviation of these averages was $0.16 \mathrm{in}$. Four of the 32 floor trusses, had an average thicknesses between $0.52 \mathrm{in}$. and $0.56 \mathrm{in}$. These measurements suggest that the minimum average thickness exceeded $1 / 2 \mathrm{in}$.

This same report stated that, on floor 23,

... truss members located adjacent to the outside walls (within $3 \mathrm{ft}$ ) are devoid of fireproofing material. Visual inspection on floor 24 was not possible, as this area still has a lowered ceiling in place.

The data in Table I-2 can be examined further to understand the variability of the fireproofing thickness in the non-upgraded locations. Figure I-7 (a) shows the average thicknesses measured on the floor trusses of floors 23 and 24. The values appear to be similar for the two locations in terms of overall average thicknesses and the variation in average thickness. A formal analysis of variance indeed indicated no statistically significant differences between the overall mean thicknesses for the two floors. Thus, the two groups of data can be combined into one. A question to be answered is whether the values of average thickness follow a normal distribution. To answer this question, histograms and normal probability plots are used. Figure I-7 (b) shows a histogram of the average thicknesses, and it appears to be non-symmetrical and skewed to the right, which is characteristic of a lognormal distribution. ${ }^{1}$

Figure $I-7(c)$ is the normal probability plot of the average thicknesses for the combined data. If the points fall approximately on a straight line, it indicates that the data are normally distributed. It is seen that there are systematic deviations of the data from the best-fit line. To examine whether the data are represented better by a lognormal distribution, the average thicknesses, in Table I-2 were transformed by taking their

\footnotetext{
1 In a lognormal distribution, the natural logarithms of the values of a variate have a normal distribution.
} 
natural logarithm. Figure I-7 (d) is a histogram of the natural logarithms of thickness, and Fig. I-7(e) is the corresponding normal probability plot. It is seen that the data are less dispersed about the straighter line, and the correlation coefficient has increased form 0.97 to 0.99 . Thus, there is some indication that the distribution of fireproofing thickness is lognormal in the non-upgraded floor trusses.

Table 1-2. Averáge fireproofing thickness from six measurements taken in 1994 on each of 16 random floor trusses on floors 23 and 24

of WTC 1.

\begin{tabular}{|c|c|}
\hline \multicolumn{2}{|c|}{ Fireproofing Thickness (in.) } \\
\hline Floor 23 & Floor 24 \\
\hline 0.60 & 0.76 \\
\hline 0.53 & 0.60 \\
\hline 0.70 & 0.90 \\
\hline 0.76 & 0.72 \\
\hline 0.88 & 0.64 \\
\hline 0.89 & 0.80 \\
\hline 0.83 & 0.68 \\
\hline 1.17 & 0.65 \\
\hline 0.88 & 0.67 \\
\hline 0.71 & 0.77 \\
\hline 0.82 & 0.96 \\
\hline 0.52 & 0.66 \\
\hline 0.69 & 0.65 \\
\hline 0.52 & 1.11 \\
\hline 0.64 & 0.95 \\
\hline 0.52 & 0.56 \\
\hline
\end{tabular}

Source: Data provided by Port Authority of New York and New Jersey.

A lognormal distribution for the average thickness of the fireproofing on the non-upgraded floor trusses is explained as follows. It is expected that the thickness of fireproofing will be highly variable due to the difficulty in spraying the material on the relatively thin members. If the overall thickness is low and the variability is high, a normal distribution would require a fraction of the surfaces to have negative values of fireproofing. If the thickness distribution is lognormal, the thickness cannot be zero, and there is a low likelihood of having thickness close to zero. If the underlying distribution of fireproofing thickness is lognormal, the average thickness overestimates the thickness expected to be exceeded with 50 percent probability, and the median is the appropriate statistic for the 50 percentile value. 

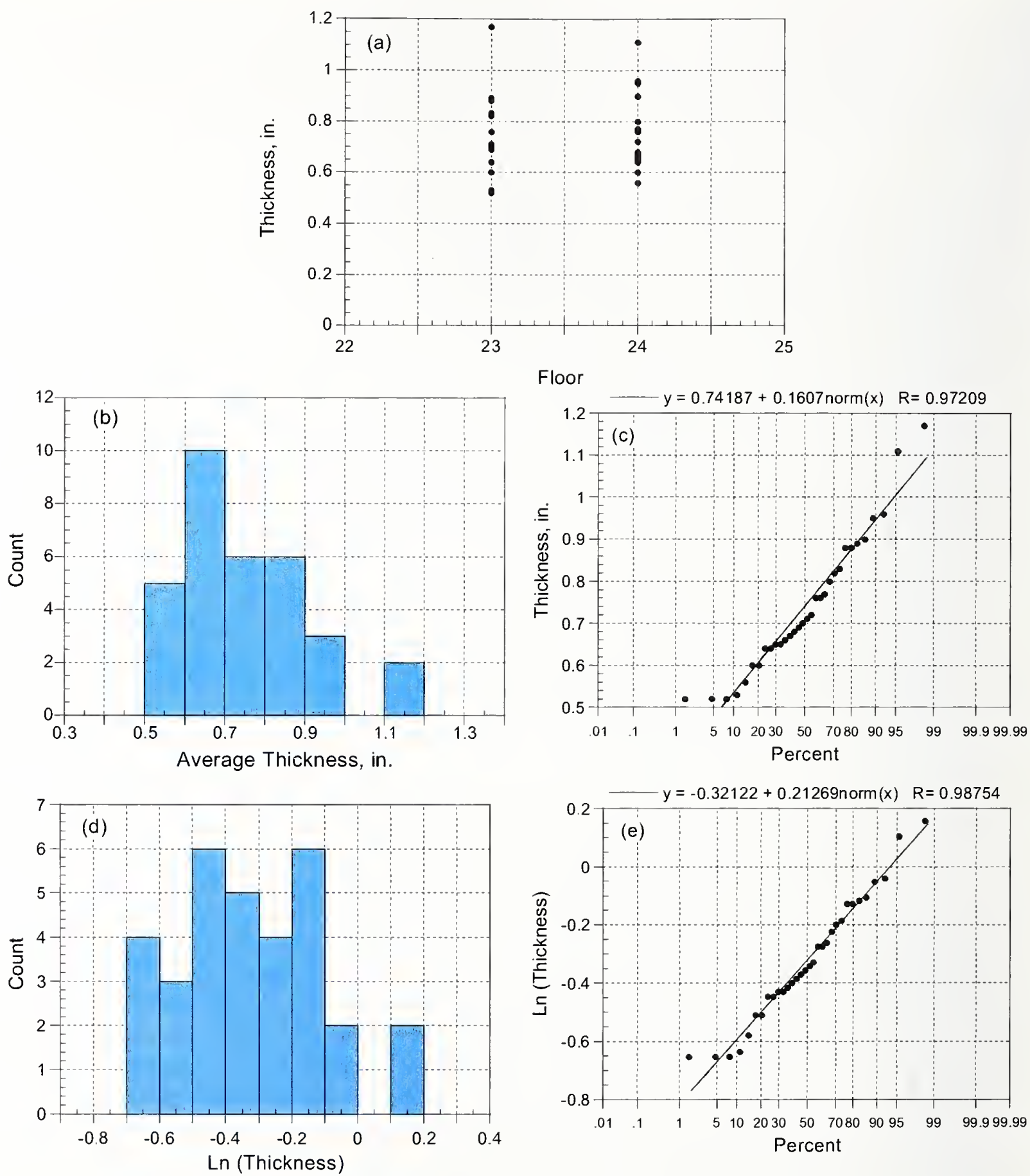

Figure 1-7. (a) Dotplot of average thickness from floor trusses for floors 23 and 24, (b) histogram of average thickness, (c) normal probability plot of average thickness, (d) histogram of natural logarithm of average thickness, and (e) probability plot of natural logarithm of average thickness. 
As stated, the standard deviation of the average thicknesses in Table I-2 is 0.16 in. Since each of the averages is based on six individual measurements, the variability in average thickness is less than the variability of the fireproofing thickness on a given element. If it is assumed that the true average thicknesses of fireproofing at the truss locations represented in Table I-2 are the same, it is possible to estimate the variability of individual measurements from the following well-known relationship:

$$
S_{\bar{X}}=\frac{S}{\sqrt{n}}
$$

where:

$$
\begin{aligned}
& S_{\bar{X}}=\text { standard deviation of the average thicknesses } \\
& S=\text { standard deviation of the individual thickness measurements } \\
& n=\text { number of measurements to obtain the average thickness }
\end{aligned}
$$

Thus, an estimate of the standard deviation of the individual measurements is $0.16 \sqrt{6} \approx 0.4 \mathrm{in}$. Since it is unlikely that there is no difference in average fireproofing thickness at different cross sections, the standard deviation of $0.4 \mathrm{in}$. is an upper limit for the variability of fireproofing thicknesses in the nonupgraded floor trusses on the basis of the information provide in Table I-2.

\section{Analysis of Photographs}

Additional data regarding the thickness of fireproofing has been gathered by evaluating photographic evidence. Although photographic evidence of the state of the fireproofing is limited, two groups of photographs have been located and used for estimating fireproofing thickness.

The first group of photographs was provided to NIST by Morse Zehnter Associates and includes images of floor trusses from WTC 1 (floors 12, 22, 23, and 27) and WTC 2 (floor 26). From this group, only photographs from floors 22, 23, and 27 of WTC 1 were analyzed. Photographs provided by Morse Zehnter Associates were taken in the mid-1990s and illustrate the fireproofing conditions prior to the upgrade carried out by the Port Authority. Thus, fireproofing thickness on the photographed trusses should be at least $1 / 2$ in. as specified by the Port Authority on October 1969.

The second group of photographs, taken in 1998, was provided by Gilsanz Murray Steficek (consulting engineers). This group illustrates the state of fireproofing after the upgrade program that was initiated in 1995. The photographs were of trusses for floor 31 and below in WTC 1.

Selection of which photographed trusses were used to estimate thickness of fireproofing was based on clarity of fireproofing edges and whether a feature of known dimensions was present. Thus, only photographs where reference measurements could be performed were used. The general approach to the analysis involved the estimation of distances based on the computed reference length per pixel. The procedure is summarized as follows:

- A feature of known dimension (based on construction drawings) that could be used as reference was located in the photograph. For example, the dimension of the bare vertical leg of a damper saddle was a dimension that could be obtained from shop drawings. 
- In the photograph, the length of the reference dimension was measured in pixels.

- The scaling factor of length per pixel was computed by dividing the known dimension in inches by the number of pixels. For example, if the vertical leg of the damper saddle was measured as 48.2 pixels in the photograph, and it is known that the actual size of the leg was $3.13 \mathrm{in}$., the scaling factor would be $3.13 \mathrm{in} . / 48.2$ pixels $=0.065 \mathrm{in} . / \mathrm{pixel}$.

- Only truss webs or struts (diagonal bar at end of truss) located near and in the same plane as the reference object were selected for analysis. This selection was made to minimize error due to perspective.

- It was assumed that the fireproofing on web bars was applied evenly around the perimeter of the bar. Based on this assumption, a "virtual" centerline along the length of the bar was drawn in the photograph.

- Lines were drawn perpendicular to the "virtual" centerline. The number of pixels along the lines from the "virtual" centerline to the edge of the fireproofing was determined from the cursor positions indicated by the software. Measurements were made at regularly spaced intervals to avoid bias. Figure I-8 is an example of a series of measurements made on a strut.

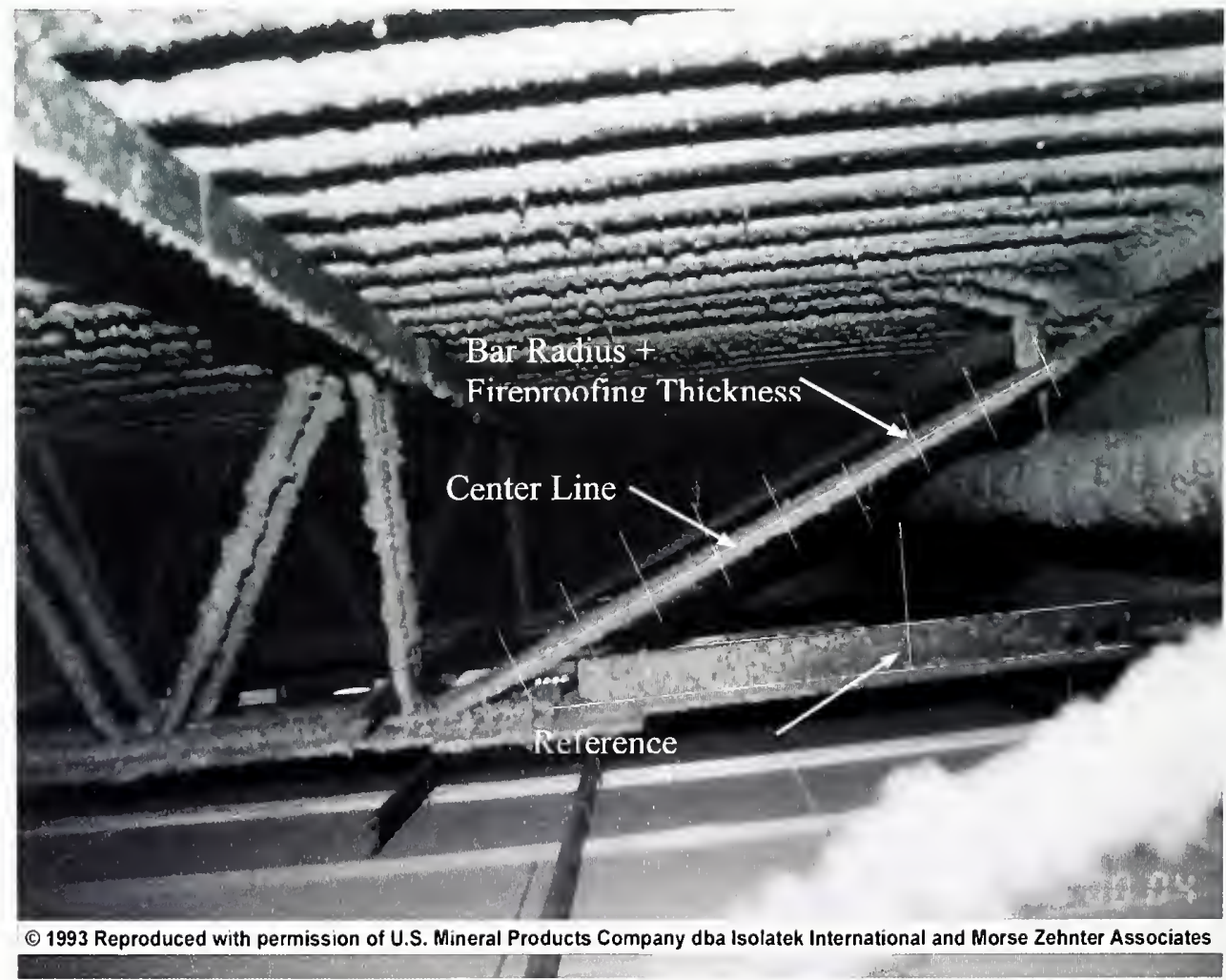

Figure 1-8. Example of measurement procedure used to estimate fireproofing thickness from photographs.

- Each measurement in pixels was multiplied by the scaling factor (in./pixel) to estimate the bar radius plus fireproofing thickness. 
- The radius of the bar was subtracted to provide the estimate of the fireproofing thickness.

It was observed that the estimated thickness of fireproofing in the non-upgraded floors tended to be larger for the webs of the main trusses. Hence estimates of fireproofing thickness were divided into three groups:

- Webs of main trusses,

- Webs of bridging trusses, and

- Diagonal strut at the exterior wall end of the truss.

No estimates of fireproofing thickness on top and bottom chords were possible using photographs. For the upgraded floors in WTC 1 that were included in the second group of photographs, only estimates of the thickness on the web bars of the main trusses were made. Figure I-9 (a) shows normal probability plots of the fireproofing thickness estimated from the photographs. It is seen that the points for the "upgraded" main trusses follow a generally linear trend, which indicates that the estimated thicknesses for the upgraded main trusses are approximately normally distributed. The estimated thicknesses from the non-upgraded floors, however, do not follow linear trends on the normal probability plot. Figure I-9 (b) shows normal probability plots of the natural logarithms of the thicknesses. The transformed values for the non-upgraded fireproofing now follow generally linear trends, which means that a lognormal distribution is more appropriate for the non-upgraded floors. This reinforces the observation noted in the previous section. Thus there is strong evidence that the original fireproofing thickness on the floor trusses follows a $\log$ normal distribution.

The average, standard deviation, and coefficient of variation were computed for the total number of measurements in each of these groups. The results are summarized as follows:

- Main trusses before upgrade: Average thickness 0.6 in., standard deviation $=0.3$ in., and coefficient of variation $=0.5$.

- Bridging trusses before upgrade: Average thickness 0.4 in., standard deviation $=0.25$ in., and coefficient of variation $=0.6$.

- Diagonal struts before upgrade: Average thickness 0.4 in., standard deviation $=0.2$ in., and coefficient of variation $=0.5$.

- Main trusses after upgrade: Average thickness 1.7 in., standard deviation $=0.4$ in., and coefficient of variation $=0.2$.

\section{Port Authority Data on Upgraded Fireproofing on Trusses}

As discussed in the May 2003 Progress Report (NIST SP 1000-3), the Port Authority provided information on fireproofing thickness from tenant alteration Construction Audit Reports prepared in 1997 to 1999. Those reports included average thicknesses of fireproofing at the "bottom of truss." In 2004, the Port Authority provided NIST reports of the individual measurements for many of the average thicknesses in the Construction Audit Reports. With the individual measurements, it is possible to investigate the 

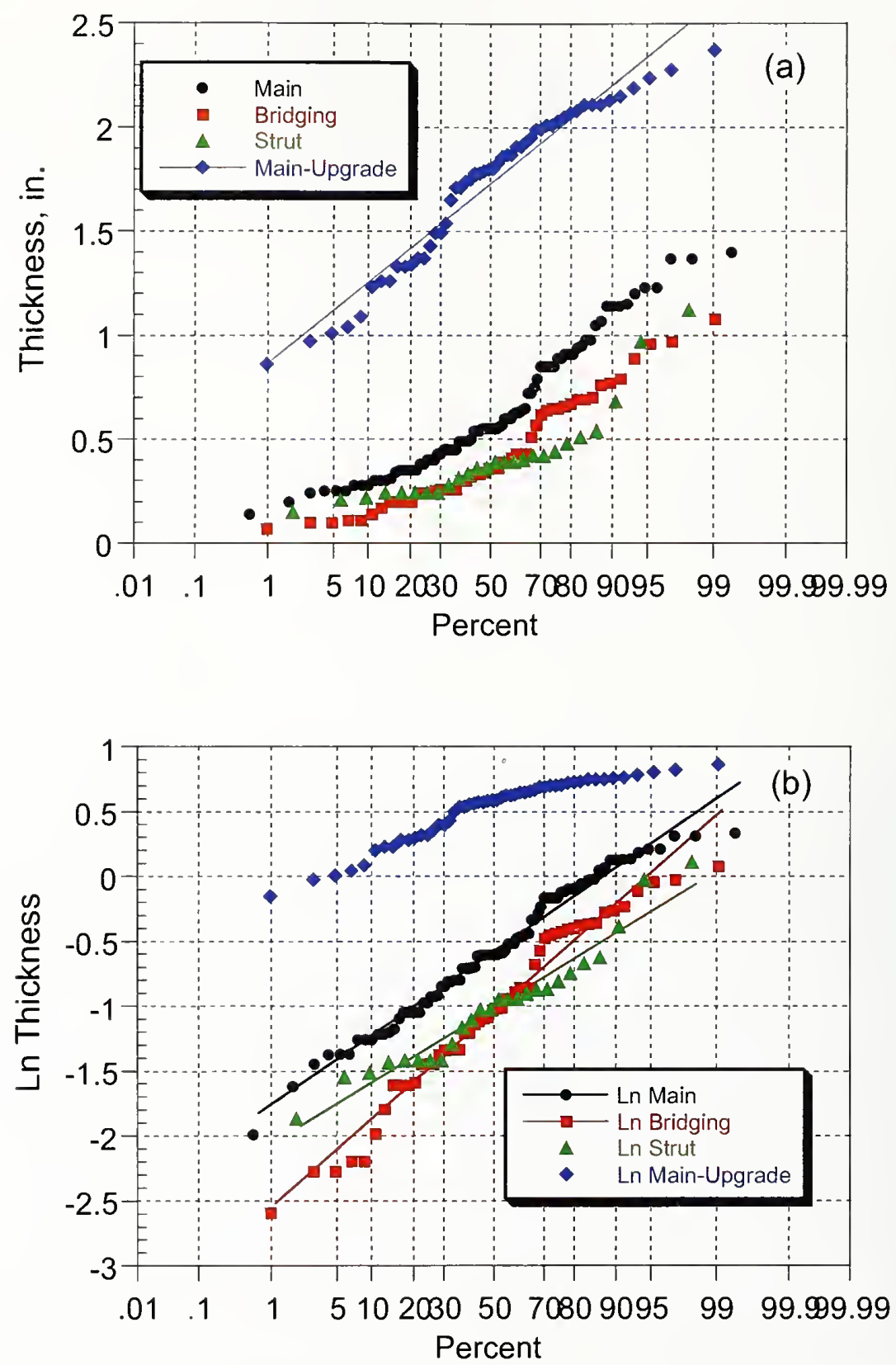

Figure 1-9. (a) Normal probability plot of estimated fireproofing thickness based on photographs, and (b) normal probability plot of natural logarithms of thickness.

variation of thickness at a cross section of a truss member and the variation in average thickness from truss to truss. To permit such analyses, only those data having the same number of individual measurements at each cross section were used. This resulted in 18 data sets for WTC 1 (including floors 93, 95, 98, 99, and 100) and 14 data sets for WTC 2 (including floors 77, 78, 88, 89, and 92).

An analysis of the individual measurements was carried out to determine the underlying distribution for the measured thicknesses. Figure I-10 (a) is a dotplot of the individual measurements in WTC 1 (144 measurements) and in WTC 2 (112 measurements). It is observed that the central values and ranges 
are similar for the two towers, and the two groups of measurements were combined into one group. Figure I-10 (b) is the histogram of the individual measurements, and Fig. I-10 (c) is the corresponding normal probability plot. A straight line fit to the normal probability plot shows a tendency of the points to deviate from the line. Figure I-10 (d) is a histogram of the natural logarithms of the individual thickness values, and Fig. I-10 (e) is the corresponding lognormal probability plot. A comparison of the probability plots shows that natural logarithms fall closer to a straight line. Thus, it appears that the thickness of the upgraded fireproofing on the floor trusses is described by a lognormal distribution. This contradicts the observation based on analysis of photographs from lower floors discussed in the previous section. The overall average thickness of the 256 individual measurements is 2.5 in. with a standard deviation of 0.6 in. Thus, the average thickness on the upgraded upper floors appears to be greater than that estimated from photographs taken on upgraded lower floors.

The overall standard deviation of 0.6 in. includes two contributions: (1) the variation of thickness at the cross section (within-truss variability), and (2) the variation of average thickness between trusses (between-truss variability). Figure I-11 shows these two components of the thickness variability for the two towers. Figures I-11 (a) and (c) show the within-truss variability, and Figs. I-11 (b) and (d) show the variation of average thickness of each truss. From analysis of variance, it was found that the within-truss standard deviation is $0.4 \mathrm{in}$., and the between-truss standard deviation is also $0.4 \mathrm{in}$. The within-truss standard deviation of $0.4 \mathrm{in}$. is similar to the standard deviation of the estimated individual thickness obtained from analysis of the photographs of upgraded main trusses.

\section{Column Fireproofing Thickness}

NIST requested that the Port Authority provide available information on the thickness of fireproofing for the exterior and interior columns of the WTC towers. Specifically, the request included the following:

- The fireproofing material used and the thickness on the various plates comprising the exterior columns and spandrels.

- The fireproofing material used and the thickness on core columns.

- Confirmation that the wide flange column sections were protected with CAFCO BLAZESHIELD Type DC/F with specified thickness of $23 / 16$ in. for sections smaller than $14 \mathrm{WF} 228$ and $13 / 16$ in. for 14WF228 and larger.

- Information on in-place fireproofing thickness.

The Port Authority replied that, due to inaccessibility of exterior columns and core columns, there were no recent records of fireproofing thickness for these elements. The only available measurements of fireproofing thickness were for beams and columns accessible within elevator shafts. The most complete data set included measurements on beams and columns taken within shaft 14/15 in WTC 1. These measurements were taken in April 1999 and included measurements from floor 1 to floor 45. The thicknesses were recorded to the nearest $1 / 8$ in., with a few thicknesses recorded to the nearest $1 / 16$ in. The columns included 10 to 18 replicate measurements, and the beams included 11 to 16 replicate measurements. 

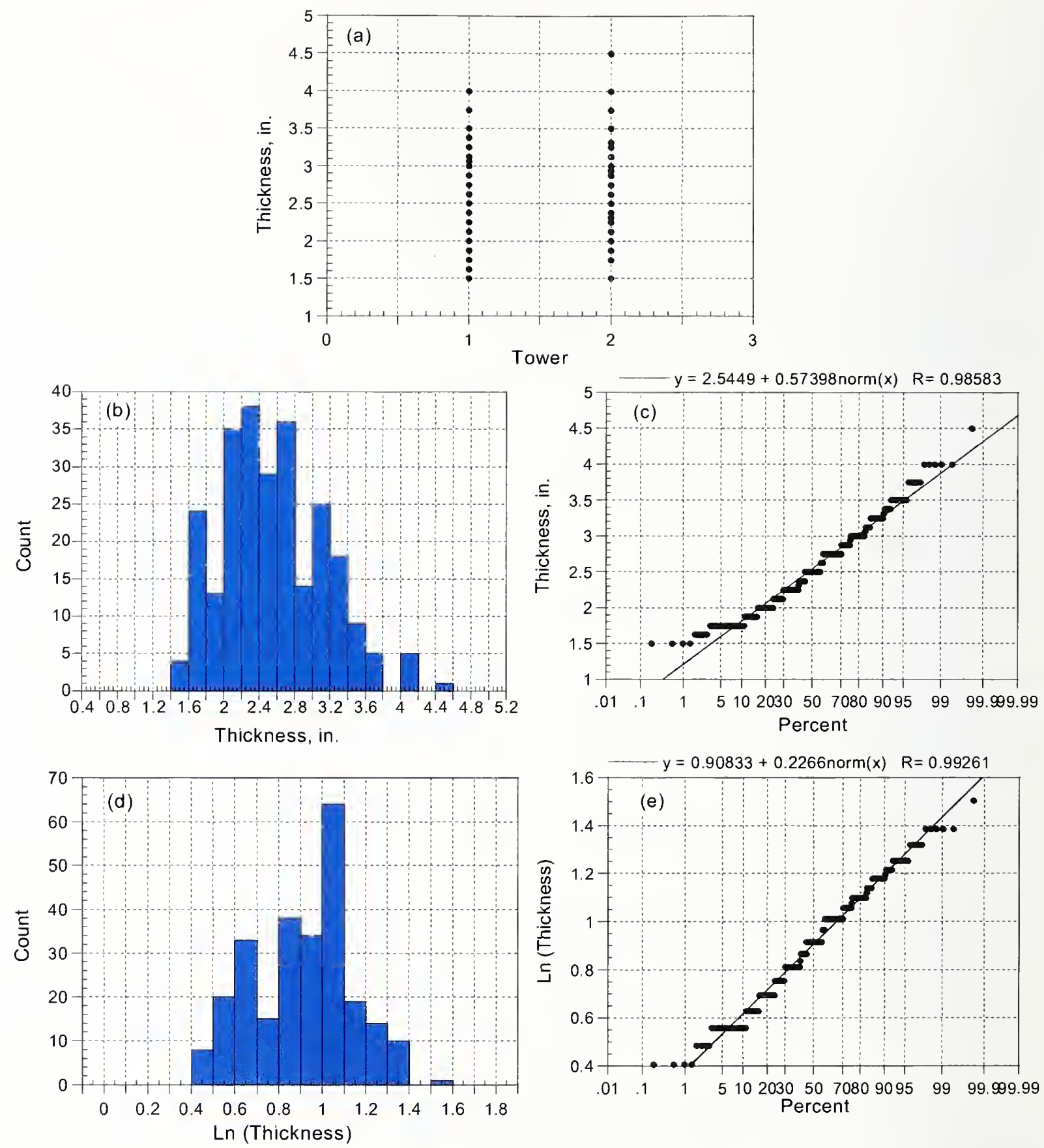

Figure 1-10. (a) Dotplot of individual thickness measurements on floor trusses from Port Authority Construction Audit Reports, (b) histogram of thickness measurements, (c) normal probability plot of thickness measurements, (d) histogram of natural logarithms of thickness measurements, and (e) normal probability plot of natural logarithm of thickness measurements. 

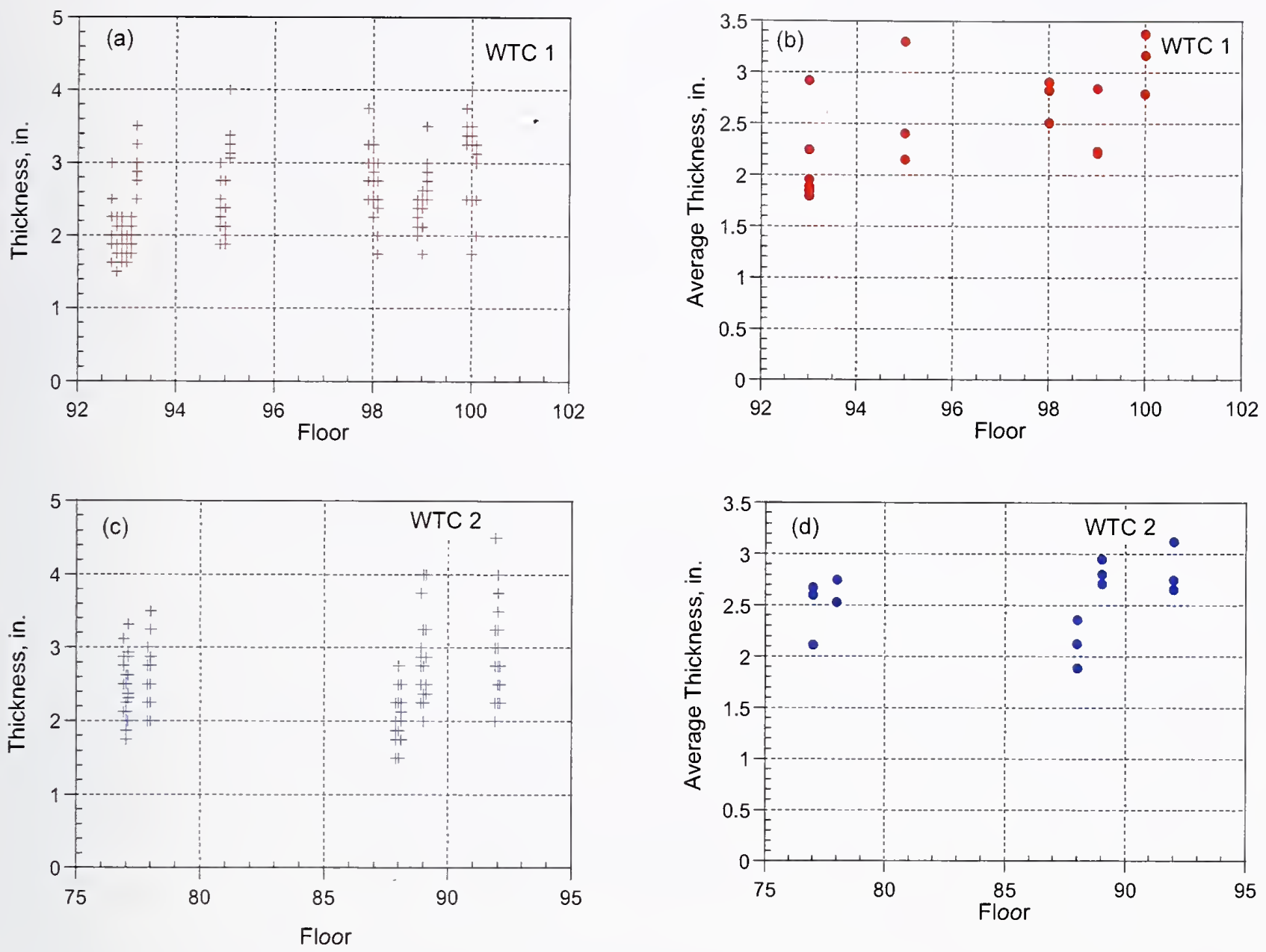

Figure I-11. Fireproofing thickness on floor trusses in upgraded portions of WTC towers: (a) individual measurements in WTC 1, (b) average thickness in WTC 1, (c) individual measurements in WTC 2, and (d) average thickness in WTC 2.

Figure I-12 (a) shows the individual and average fireproofing thickness on the core columns. Analysis of variance indicated no statistically significant differences among the average values and all data were pooled together. The average thickness for the columns is 0.82 in., the standard deviation is 0.20 , and the coefficient of variation is 0.24 . The information from the Port Authority indicated that the "minimum thickness required" for the columns was 0.5 in. Figure $\mathrm{I}-12$ (b) is the normal probability plot of the individual thickness measurements. Because most of the thicknesses were reported to the nearest $1 / 8$ in., the points are staggered instead of uniformly distributed. The plot, however, shows that the points follow a linear trend, and it appears that the thickness of the fireproofing on the core columns could be described by a normal distribution. Figures I-12 (c) and (d) shows the corresponding plots for the thickness of fireproofing on the beams. The average thickness for the beams is 0.97 in., the standard deviation is $0.21 \mathrm{in}$. and the coefficient of variation is 0.21 . The information from the Port Authority indicated that the "minimum thickness required" for the beams was $0.75 \mathrm{in}$. 

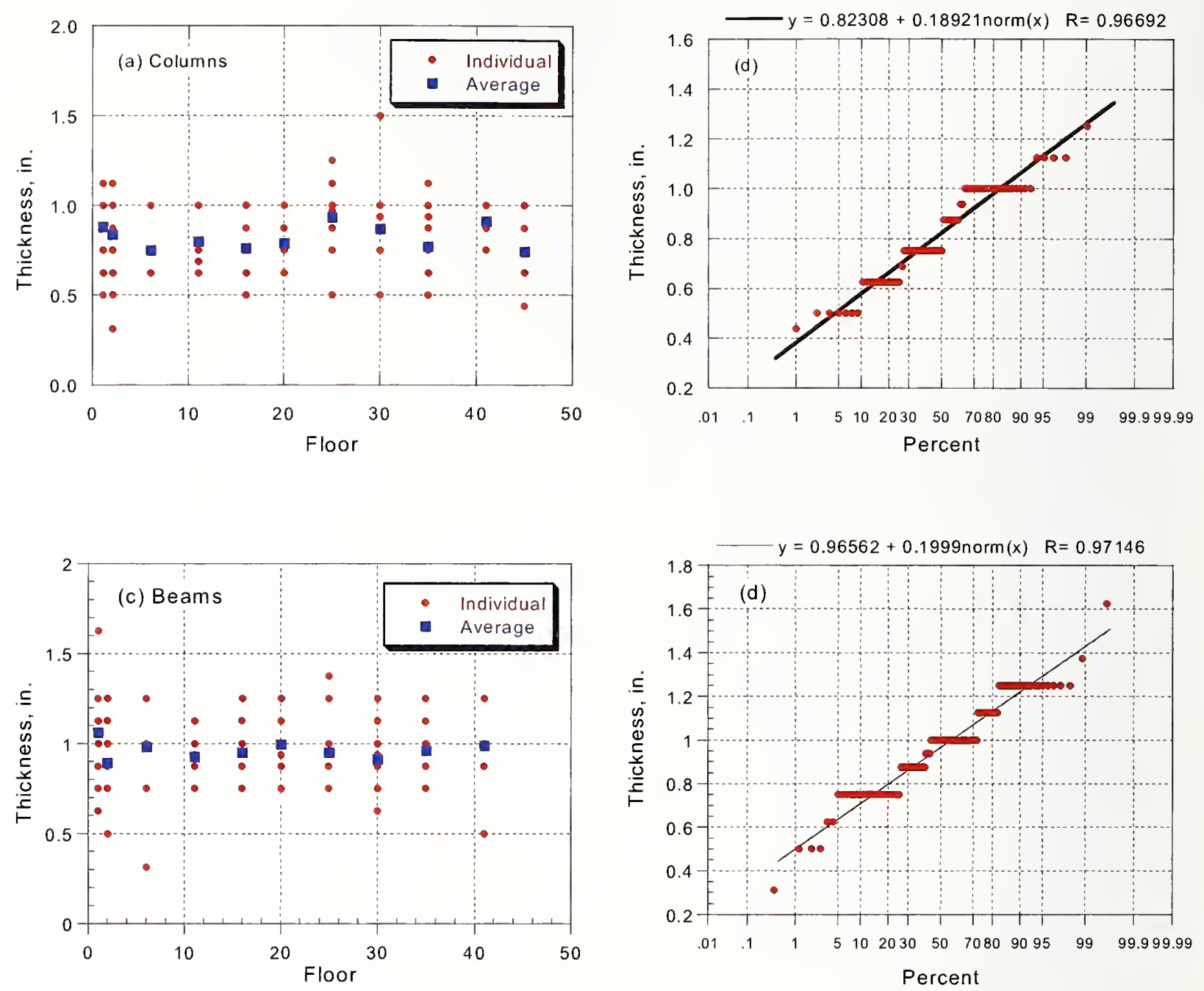

Figure I-12. (a) Individual and average thickness for core columns, (b) normal probability plot of individual measurements on columns, (c) individual and average thickness for core beams, and (d) normal probability plot of individual measurements on beams.

As might be expected, the variation in thickness of fireproofing for the beams and columns is lower than the variation observed in the floor trusses. The planar surfaces of the beams and columns result in more uniform application of the sprayed fireproofing than for the slender truss members. This results in reduced differences in the average thickness of fireproofing on different members and less variability within a member.

\section{I.3.4 Equivalent Thickness}

The sensitivity study summarized in Section I.2 indicated that variation in the thickness of fireproofing reduced the "effective thickness" of the fireproofing. It would be impractical to attempt to account for the variation in fireproofing thickness in the thermal modeling by introducing variable thickness fireproofing in the finite-element models. As an alternative, it was decided to attempt to determine the "equivalent uniform thickness" of fireproofing that would result in the same thermo-mechanical response of a 
member as variable thickness fireproofing. An approach similar to the methodology described in Section I. 2 was used to model a 1 in. diameter by $60 \mathrm{in.} \mathrm{long} \mathrm{bar} \mathrm{with} \mathrm{fireproofing} \mathrm{and} \mathrm{subjected} \mathrm{to} \mathrm{the}$ heat flux arising from a $1,100{ }^{\circ} \mathrm{C}$ fire. The bar was subdivided into $0.6 \mathrm{in}$. long elements, so that there were 100 elements along the length of the bar. The thermal history of the bar was calculated, and that history was used to calculate the length change of the unrestrained bar under a tensile stress of 12,500 psi. The bar was assumed to be similar to the steel used in the floor trusses, and the temperature dependence of the coefficient of thermal expansion and the modulus of elasticity were based on NIST measurements.

The fireproofing thickness in the models was based on the measurements summarized in the previous section for the web bars of main trusses in the original condition and after the upgrade. Specifically, the following target values were investigated:

- Original: average thickness $=0.75$ in., standard deviation $=0.3$ in., lognormal distribution.

- Upgrade: average thickness $=2.5$ in., standard deviation $=0.6$ in., lognormal distribution.

The variation of fireproofing thickness along the length of the bar was established by using a psuedo random number generator to select values from a lognormal distribution with central value and dispersion consistent with the above average values and standard deviation. Three sets of random data were generated for each condition.

When the randomly selected thicknesses of each element were applied to the bar, it resulted in sudden changes in fireproofing thickness along the length of the bar. This resulted in a "rough" surface texture as shown by the dotted thickness profile in Fig. I-13 (a). It was felt that this rough texture (see also Fig. I-1 (c) might not be representative of actual conditions, so an alternative approach was to use 5-point averaging to reduce the roughness of the fireproofing profile. The solid line in Fig. I-13 (b) shows such a "smooth" profile. The two profiles in Fig. I-13 (a) have approximately the same average value and standard deviation and have similar cumulative distribution of fireproofing thickness as shown in Fig. I-13 (b).

As stated, the calculated thermal histories of the bar elements were used to calculate the unrestrained length change of the bar due to thermal expansion and an applied stress of $12,500 \mathrm{psi}$. Work is currently underway to examine the performance of the bar under fully restrained conditions in which the induced stress history is computed. For comparison, the deformation of the bar with different but uniform thickness of fireproofing was calculated. The "equivalent thickness" was taken as the uniform thickness that resulted in similar deformation as under the variable thickness conditions. Figure I-13 (c) shows the results of these calculations for the original fireproofing. The three continuous curves are the deformation-time relationships for uniform thickness of 0.4 in., 0.5 in., and 0.6 in. The solid symbols represent the results for three cases with "rough" texture, and the open symbols are for the "smooth" texture. The following values summarize the six variable thickness profiles:

- Rough 1: average $=0.79 \mathrm{in}$., standard deviation $=0.29 \mathrm{in}$.

- $\quad$ Rough 2: average $=0.77$ in., standard deviation $=0.27$ in

- Rough 3: average $=0.79$ in., standard deviation $=0.31$ in 


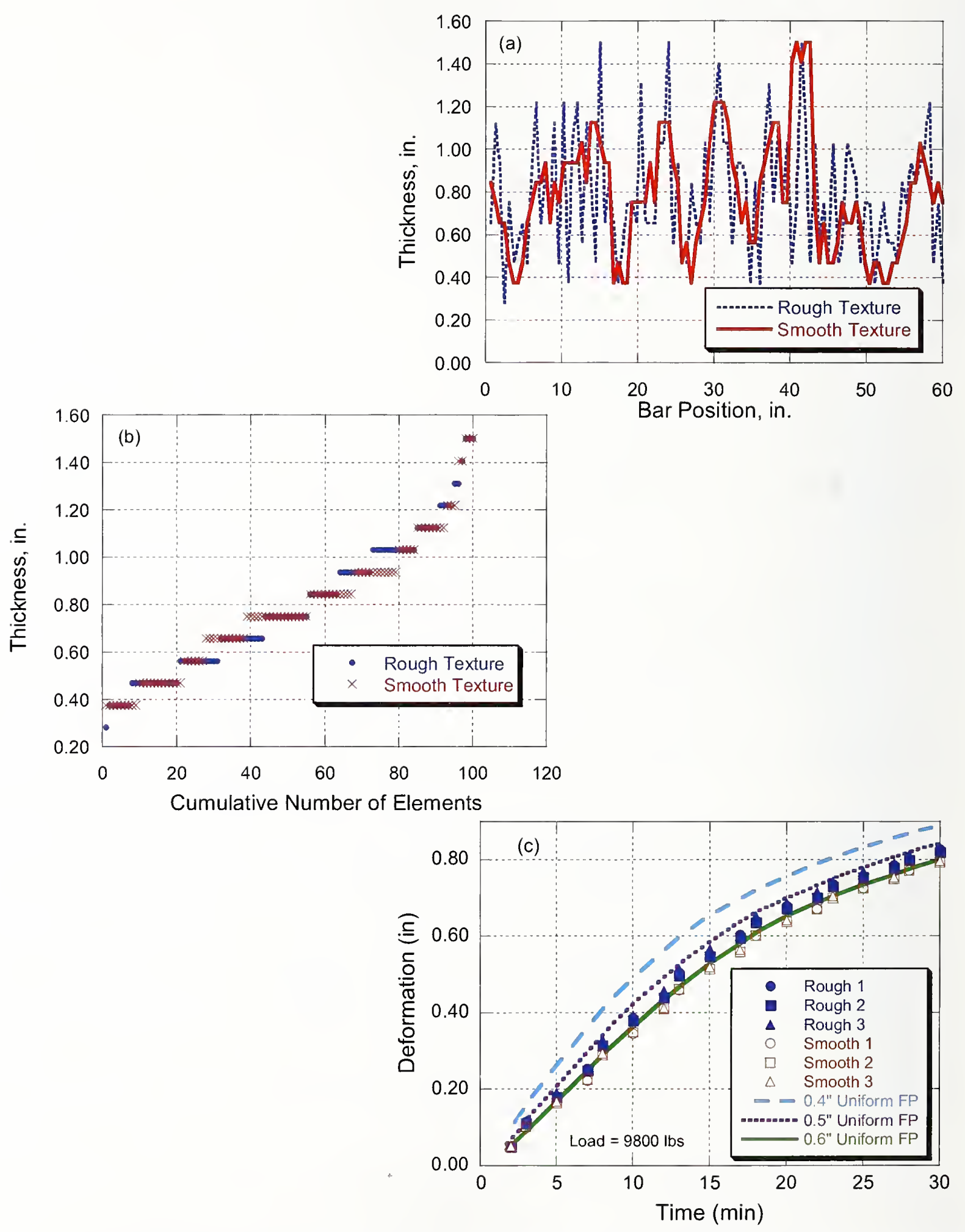

Figure 1-13. (a) Randomly generated thickness profiles with average thickness of 0.75 in. and standard deviation of $\mathbf{0 . 3}$ in., (b) cumulative element size, and (c) deformation of 1 in. bar compared with deformation for uniform thickness of fireproofing. 
- Smooth 1: average $=0.79$ in., standard deviation $=0.28$ in

- Smooth 2: average $=0.78$ in., standard deviation $=0.31$ in.

- Smooth 3: average $=0.78$ in., standard deviation $=0.32$ in.

Figure I-13 (c) shows that the "rough" texture reduces the effectiveness of the fireproofing by a small amount compared with the "smooth" texture. As noted above, it is believed that the "smooth" texture is more representative of the actual conditions. On the basis of these analyses, it is concluded that fireproofing with an average thickness of 0.75 in. and a standard deviation of 0.3 in. provides equivalent protection to 0.6 in. of uniform thickness.

The results for the upgraded fireproofing are shown in Fig. I-14. Only the "smooth" texture was used, and the values for the three cases are as follows:

- Case 1: average $=2.50$ in., standard deviation $=0.71$ in.

- Case 2: average $=2.43$ in., standard deviation $=0.51$ in.

- $\quad$ Case 3: average $=2.55$ in., standard deviation $=0.63$ in.

Figure I-14 (a) shows the three profiles, and Fig. I-14 (b) shows the normal probability plots of thickness values. Because the three randomly generated profiles do not have the same averages and dispersions, the responses show more scatter than in Fig. I-13 (c). On the basis of these analyses, it is concluded that an average thickness of fireproofing of $2.5 \mathrm{in}$. with a standard deviation of $0.6 \mathrm{in}$. is equivalent to $2.2 \mathrm{in}$. of uniform thickness.

\subsubsection{Thickness of SFRM for Use in Analyses}

Analyses of available data on fireproofing thickness and thermal modeling revealed the following:

- From measurements of fireproofing thickness, the average values exceeded the specified thickness.

- Fireproofing thickness was variable, and the distribution of thickness in the floor trusses appears to be described best by a lognormal distribution.

- The standard deviation of fireproofing thickness on the trusses varied between about $0.3 \mathrm{in}$. to 0.6 in.

- The standard deviation of fireproofing on columns and beams from the core tended to be lower, with a value of $0.2 \mathrm{in}$. for the available data.

- No information is available on the fireproofing thickness on the exterior columns and spandrel beams.

- Variation in thickness reduces the effectiveness of fireproofing, and the equivalent uniform thickness is less than the average thickness. 

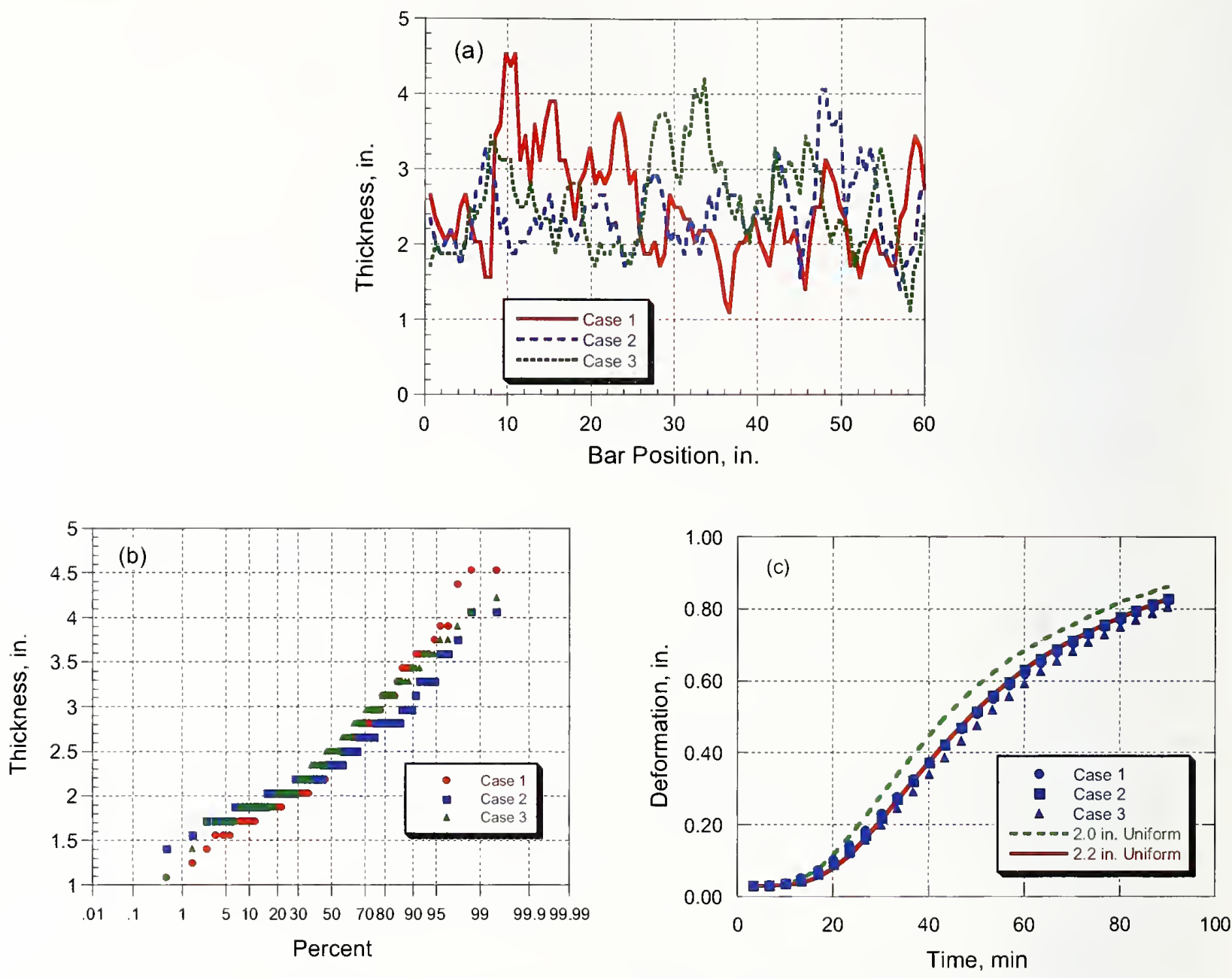

Figure I-14. (a) Randomly generated thickness profiles with average thickness of 2.5 in. and standard deviation of 0.6 in., (b) normal probability plots of thickness values, and

(c) deformation of 1 in. bar compared with deformation for uniform thickness of fireproofing.

Based on the above findings, the following uniform thickness for the undamaged fireproofing will be used in calculating thermal response under various fire scenarios:

- Original fireproofing on floor trusses: 0.6 in.

- Upgraded fireproofing on floor trusses: 2.2 in.

- Fireproofing on other elements: the specified thickness.

The choice of specified thickness for those members lacking data is justified by offsetting factors as follows: (1) measured average thicknesses exceed specified values, and (2) variation in thickness reduces the effectiveness of fireproofing. 


\section{I.4 THERMAL PROPERTIES}

Based on the information provided by the manufacturers, three SFRMs have been identified in WTC 1, 2, and 7: (1) CAFCO BLAZE-SHIELD Type DC/F, (2) CAFCO BLAZE-SHIELD Type II, and (3) Monokote MK-5. Of the three SFRMs, only CAFCO BLAZE-SHIELD Type II is currently sold in the U.S., and CAFCO BLAZE-SHIELD Type DC/F is sold in Canada.

CAFCO BLAZE-SHIELD Type DC/F is manufactured by Isolatek International (Stanhope, New Jersey) and was used in the interior columns, floor systems, and the exterior faces of the exterior columns of WTC 1 and WTC 2. CAFCO BLAZE-SHIELD Type II, also from Isolatek, was used in subsequent retrofit of WTC 1 floor systems. CAFCO BLAZE-SHIELD Type DC/F and Type II are portland cementbased products. Monokote MK-5 a gypsum-based SFRM, was manufactured by W.R. Grace and Co. (Cambridge, Massachusetts) and used in WTC 7. W.R. Grace stopped the production of Monokote MK-5 in the 1980s. In addition to these three SFRMs, vermiculite plasters, manufactured by W.R. Grace until the 1970s, were used on the interior faces of the exterior columns of WTC 1 and WTC 2.

To provide thermophysical property data for the modeling effort in fire-structure interaction, the thermal conductivity, specific heat and density of each SFRM were determined as a function of temperature up to $1200{ }^{\circ} \mathrm{C}$. Tests were performed by Anter Laboratories, Inc. in Pittsburgh, PA through an open-bid contract. Anter Laboratories is an ISO 9002 certified company.

Samples of CAFCO BLAZE-SHIELD Type DC/F and Type II were prepared by Isolatek, Inc. in Stanhope, New Jersey, and sample of Monokote MK-5 were prepared by W.R. Grace and Co. in Cambridge, Massachusetts according to their respective application manuals. Since Monokote MK-5 is no longer on the market, it was specially manufactured by W.R. Grace according to the original MK-5 formulation. The samples were made from the same batch of raw material, shipped to NIST for examination and documentation, and sent to Anter Laboratories for testing. The sample is $9 \mathrm{in}$. long, $4.5 \mathrm{in}$. wide, and $3 \mathrm{in}$. thick. Three samples of each material were sent for testing. Two of them were used for the thermal conductivity measurements, and the third was used to prepare specimens for the other measurements involved.

\section{I.4.1 Thermal Conductivity Measurements}

The thermal conductivity measurements were performed according to ASTM C 1113 Test Method for Thermal Conductivity of Refractories by Hot Wire (Platinum Resistance Thermometer Technique). This test method is based on heating two specimens with a platinum wire placed between them. The thin platinum wire serves not only as a heater, but also as a temperature sensor, since the variation of its electrical resistance during the test is converted into variation of temperature. Thermal conductivity is calculated based on the rate of temperature increase of the wire and power input. It was reported that substantial shrinkage during the measurements occurred for the three materials. The two MK-5 specimens shrunk, exposing the platinum wire positioned between them. For this reason, no thermal conductivity measurement could be performed for this material at $1,200{ }^{\circ} \mathrm{C}$. Figure $\mathrm{I}-15$ (a) shows preliminary results for thermal conductivity as a function of temperature. The results show similar trends of increased thermal conductivity with increasing temperature; however, the Monokote MK-5 specimens had a different behavior than CAFCO BLAZE-SHIELD Type DC/F and Type II at temperatures above $500^{\circ} \mathrm{C}$. 

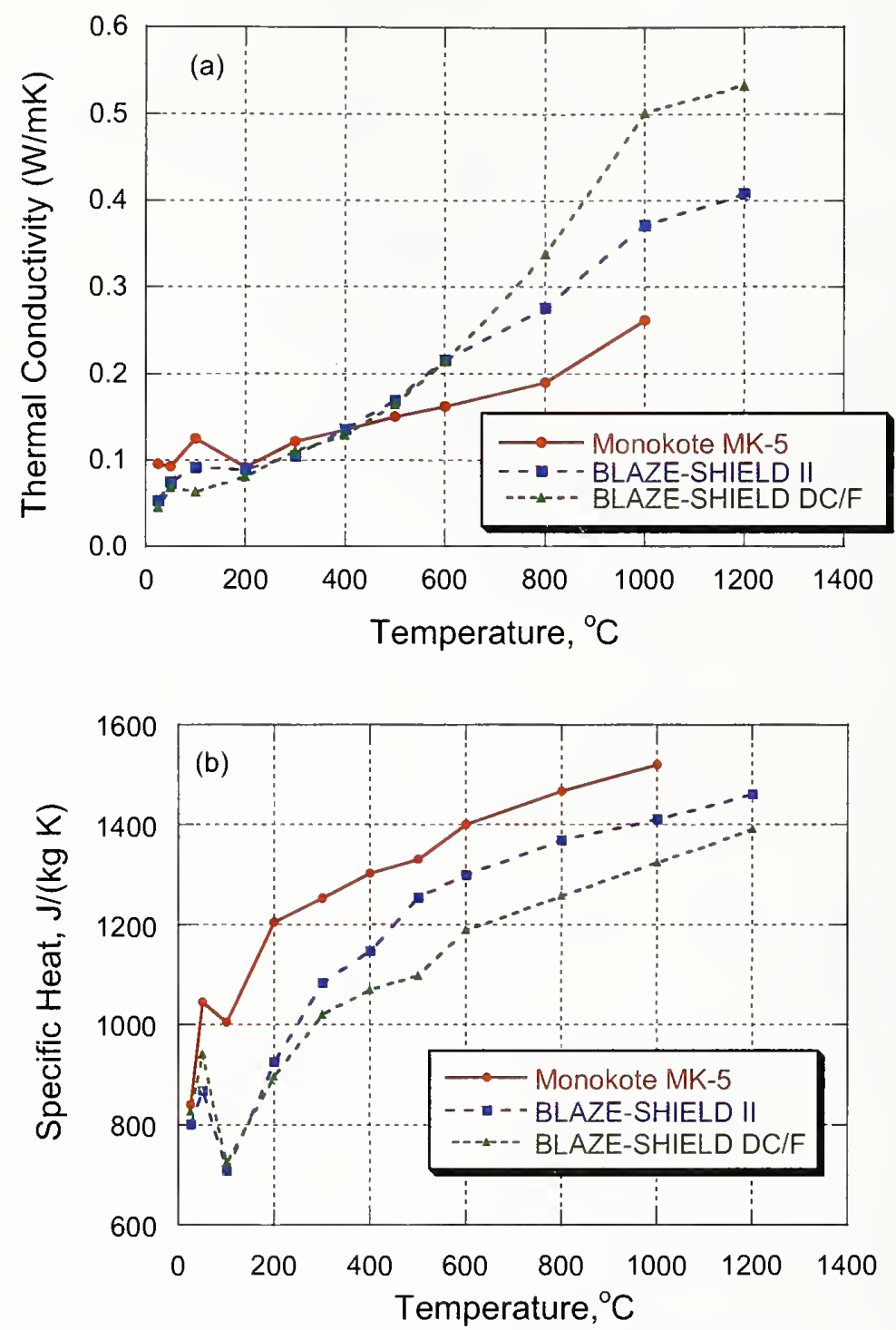

Figure 1-15. Preliminary test results: (a) thermal conductivity as a function of temperature, and (b) specific heat as a function of temperature.

\section{I.4.2 Specific Heat Measurements}

For the specific heat capacity measurements, the same instrument (Unitherm Model QL-3141) was used with a slight modification. A thermocouple was added to the system and mounted on the specimen, parallel with the platinum wire at a known distance from the thermocouple. The test was performed in a similar manner as the thermal conductivity measurements, but from the thermocouple output the thermal diffusivity of the material was derived. Knowing the thermal conductivity, the thermal diffusivity, and the density calculated from the thermal expansion results and the thermogravimetric analysis, the specific heat of the material was calculated. Figure I-15 (b) shows preliminary results for specific heat as a function of temperature. It is seen that the materials had similar increasing trends with temperature, but the actual values differed. 


\section{I.4.3 Density Measurements}

Densities of the samples were not measured directly (except at room temperature) but were calculated from TGA (thermal gravimetric analysis) and thermal expansion measurements. The TGA tests were performed according to ASTM Test Method E1131 using an Orton Model ST-736 TGA instrument. The thermal expansion tests were performed according to ASTM Test Method E228 using a Unitherm Model 1161 instrument. Since the materials were not isotropic, separate tests were performed for the $\mathrm{X}$ and $\mathrm{Z}$ orientations. It was assumed that the $\mathrm{X}$ and $\mathrm{Y}$ directions had the same thermal expansion. The $\mathrm{Z}$ direction was defined as the direction perpendicular to the fibrous strands in the specimens. The specimens were tested from room temperature to $1,200{ }^{\circ} \mathrm{C}$ at a heating rate of $2{ }^{\circ} \mathrm{C} / \mathrm{min}$. All of the specimens shrank during the tests and, in all cases, lost contact with the pushrod before reaching the maximum test temperature.

From the thermal expansion test results, the change in volume for each material was calculated at each temperature of interest. The density values were calculated from the results of the TGA and thermal expansion.

\section{I.5 RESPONSE TO IMPACT}

In order to estimate the extent of damage or loss of SFRM due to aircraft impact, the detailed finite element analysis of aircraft impact into the WTC towers, conducted within the framework of Project 2 of the investigation, will provide the following information:

Debris Field - A database and graphics of the major fragments of the aircraft and destroyed structural components of the towers, including their mass, approximate size, speed, and trajectory will be developed in the global analysis of aircraft impact into WTC 1 and WTC 2. The trajectory of each fragment will consist of the initial point of entry, point of exit or resting place. This debris field database will be used to estimate which areas within the impacted floors would likely have lost their fireproofing due to direct impact by debris.

Deformations and Accelerations - Estimates of accelerations and deformations, including localized effects, as a function of time on steel members in each of the two towers will be developed in the global analysis of the aircraft impact. Accelerations will be determined at representative locations on the floor truss systems and columns in the impact-affected zones of both towers (floors 93 to 98 of WTC 1 and floors 78 to 83 of WTC 2). These accelerations will be compared with the threshold values estimated from the adhesion and cohesion properties of SFRM developed in the experimental and analytical study presented below to estimate the likely extent of damage to the fireproofing on the columns and floor systems.

Preliminary results from the subassembly impact analysis of an aircraft engine into a strip of the towers with a width and height of single exterior panel (three exterior columns width and three floor height) extending all the way through the core indicate that the accelerations on the lower chords of floor trusses will need further analysis to account for high frequency vibrations and the short-duration sharp peaks in the computed acceleration time-histories and their effects on damage to SFRM. One possible approach is to low-pass filter the acceleration records to remove these high-frequency vibrations. Another approach is to develop "shock spectra" for a number of steel members with fireproofing configurations using finite 
element analysis to determine, for a given frequency, the acceleration amplitude that is needed to dislodge the fireproofing based on its adhesive and cohesive strength. The shock spectra will then be compared with spectra of the calculated acceleration time-histories to estimate the extent of damage to the fireproofing.

\section{I.5.1 Mechanical Properties of SFRM}

The purpose of these tests is to develop a rational basis for estimating the extent of loss of SFRM as a result of impact loads on protected members. Tests will (1) determine the mechanical properties of CAFCO BLAZE-SHIELD Type DC/F, and (2) verify models for estimating loss of fireproofing when a protected member is subjected to impact-induced vibration. The mechanical properties to be measured are:

- SFRM cohesive strength, and

- SFRM adhesive strength to steel substrates with and without primer.

The adhesive and cohesive strengths will be measured for static loads, as described below for Phase I tests. The tests will be done on $1 / 4$ in. thick steel plate specimens, with and without primer (Tnemec Series 10 red primer), and for nominal SFRM thickness of 3/4 in. and 1 1/2 in. Specimens are fabricated and testing will be done during June and July, 2004.

From the measured strength properties, estimates will be made of the local accelerations required to damage or dislodge the SFRM, as described below. These estimates will be verified by impact tests of plates and bars covered with SFRM and instrumented with accelerometers, as described in the Phase II tests.

\section{Phase I-Tensile Pull-off Test to Measure Adhesive Bond Strength and Cohesive Strength}

Specimen-Steel plates (8 by 16 by $1 / 4$ in.) with CAFCO BLAZE-SHIELD Type DC/F and nominal thickness of $3 / 4$ in. and 1 1/2 in.

\section{Pull-Off Test Procedure (see Fig. I-16)}

- Using a fine-tooth saw, cut into SFRM applied to plate to obtain $23 / 4$ in. square test specimens to ensure that the area resisting the applied load is well defined.

- Affix aluminum plates with two-component adhesive.

- Allow adhesive to cure.

- Measure force required to pull off the plate.

- Record load and note failure mode (cohesive, adhesive, mixed). 


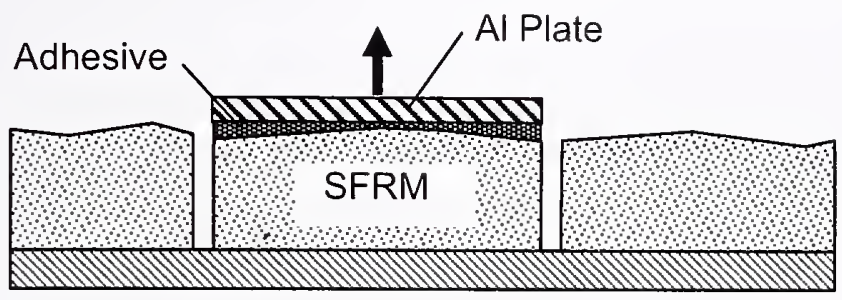

Figure I-16. Pull-off test of SFRM applied to steel plate.

If all failures are adhesive, the cohesive strength will be determined by bonding the SFRM block to a steel plate with adhesive and repeating the test.

\section{Phase II-Verification of Models to Predict Dislodgement of SFRM}

Impact tests of plate and bar specimens will be done to determine the impact loads needed to produce different levels of accelerations. Plates and bars with SFRM will be subjected to different levels of impact until the SFRM is dislodged. Two simplified models will be used to estimate the relationships between material strengths and impact required to dislodge the SFRM. Model predictions will be compared with test results.

\section{CASE 1: Planar Element}

The simplified model considers the substrate and SFRM as rigid bodies. The SFRM would dislodge when the inertial force exceeds the smaller of the adhesive bond strength or cohesive strength. Figure I-17, shows the free body of the fireproofing being acted upon by its inertial force and the adhesive force. The acceleration to dislodge the SFRM is:

$$
a=\frac{f_{b}}{\rho t}
$$

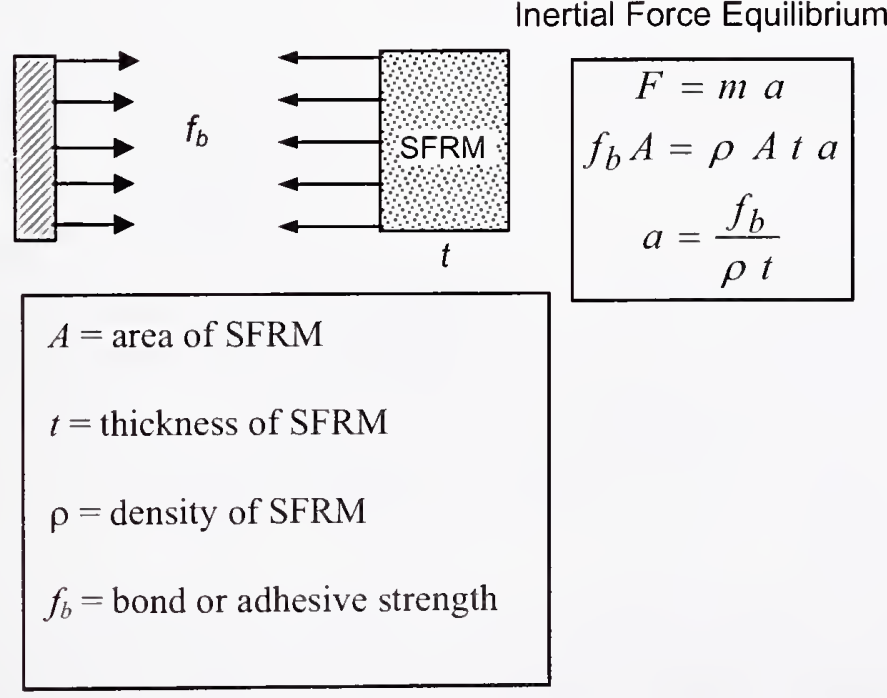

Figure 1-17. Derivation of acceleration to dislodge SFRM from planar substrate. 
where:

$$
\begin{aligned}
& f_{b}=\text { bond or adhesive strength } \\
& t=\text { thickness of SFRM } \\
& \rho=\text { density of SFRM }
\end{aligned}
$$

For example, for an SFRM with cohesive and adhesive strength of 150 psf, a density of $15 \mathrm{pcf}$, and an applied thickness $\mathrm{t}=1$ in., we would find that $a=119 \mathrm{~g}$, where $\mathrm{g}$ is the gravitational acceleration. This shows that acceleration on the order of $100 \mathrm{~g}$ would be required to dislodge this SFRM from a planar surface.

\section{CASE 2: Encased Round Element}

Again, a rigid body model is used. In this case, the SFRM would mobilize its cohesive tensile strength, $f_{t}$, and adhesive bond strength, $f_{b}$. Figure $\mathrm{I}-18$ shows the derivation for the relationship between material strengths and acceleration to dislodge the SFRM from a round bar. The required acceleration is as follows:

$$
a=\frac{4 f_{t}\left(d_{0}+(\alpha-1) d_{i}\right)}{\left(d_{0}^{2}-d_{i}^{2}\right) \rho \pi}
$$

where:

$$
\begin{aligned}
& f_{t}=\text { cohesive tensile strength of SFRM } \\
& d_{0}=\text { outside diameter of SFRM }
\end{aligned}
$$

Inertial Force Equilibrium

$$
\begin{aligned}
& \text { Mass }=m=\pi \frac{\left(d_{o}^{2}-d_{i}^{2}\right)}{4} \rho \\
& F=f_{t}\left(d_{o}-d_{i}\right)+f_{b} d_{i} \\
& \text { Let } f_{b}=\alpha f_{t} \\
& F=f_{t}\left(d_{o}+(\alpha-1) d_{i}\right)=\pi \frac{\left(d_{o}^{2}-d_{i}^{2}\right)}{4} \rho a \\
& a=\frac{4 f_{t}\left(d_{o}+(\alpha-1) d_{i}\right)}{\left(d_{o}^{2}-d_{i}^{2}\right) \rho \pi}
\end{aligned}
$$

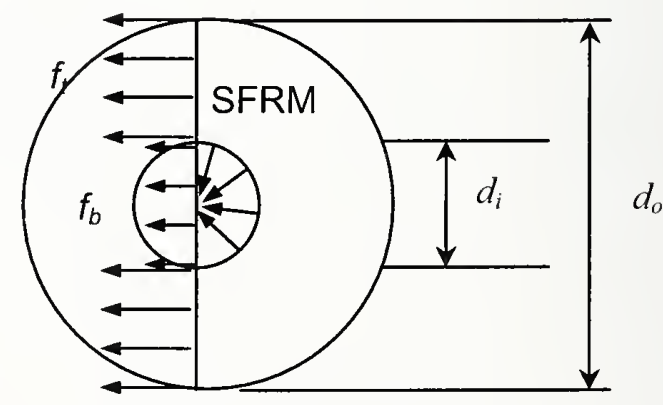

Figure I-18. Derivation of acceleration to dislodge SFRM surrounding a round bar. 


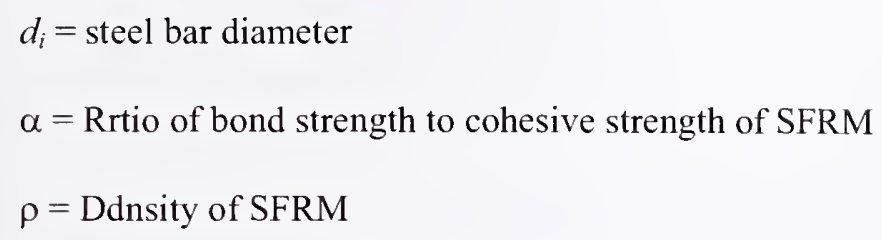

For example, if the steel bar has a diameter of $d_{i}=1 \mathrm{in}$., the SFRM has an outside diameter of $d_{0}=2$ in., a density $\rho=15$ pcf, a cohesive tensile strength of $f_{t}=300 \mathrm{psf}$, and a bond strength to cohesive strength ratio of $\alpha=0.5$, we would find that an acceleration of $\mathrm{a}=152 \mathrm{~g}$ is required to dislodge the SFRM from the bar.

\section{I.6 SUMMARY}

This appendix has focused on conditions of the fireproofing (or SFRM) in the WTC towers before and after aircraft impact. Results of simplified finite-element simulations of heat transfer under fire conditions have shown that variability in thickness of fireproofing reduces the effectiveness of the fireproofing so that protection is less than implied by the average thickness of the fireproofing. As a result, the NIST-led investigation sought available information on the in-place condition of the SFRM used in the WTC towers. Limited information was provided by the Port Authority in the form of thickness measurements taken at various times during the 1990s. Additional information was obtained from photographs of floor trusses provided to NIST. Analysis of the data indicated that fireproofing thickness was variable, as would be expected for application to floor truss members with small cross sections. Based on analyses of the available data, the following values were taken to be representative of the SFRM thickness on the floor trusses:

- Original SFRM: Average thickness of 0.75 in. with a standard deviation of 0.3 in. (coefficient of variation $=0.40$ )

- Upgraded SFRM: Average thickness of $2.5 \mathrm{in}$. with a standard deviation of $0.6 \mathrm{in}$. (coefficient of variation $=0.24$ )

Based on finite-element simulations of a 1 in. round bar covered with SFRM having lognormal distributions for thickness that are consistent with the above values, it is concluded that the original fireproofing on the floor trusses is equivalent to a uniform thickness of $0.6 \mathrm{in}$. and the upgraded fireproofing is equivalent to a uniform thickness of $2.2 \mathrm{in}$.

No information is available on in-place conditions of the fireproofing on the exterior columns and spandrel beams, and little information is available on the conditions of fireproofing on core beams and columns. In subsequent thermal analyses, the fireproofing on these elements will be taken to have uniform thicknesses equal to the specified values. This assumption is believed to be justified by the offsetting factors of measured average thicknesses tending to be greater than specified thicknesses and the reduced effectiveness of a given average thickness of fireproofing due to thickness variability.

Another objective of this appendix is to review the methodology that will be used to estimate how much of the SFRM may have dislodged as result of aircraft impact. Simple static models have been developed for an order of magnitude estimate of the acceleration that would be required to dislodge the SFRM. Based on these models and assumed, but representative, values of density and strength (adhesive and 
cohesive), it is estimated that acceleration on the order of $100 \mathrm{~g}$ to $150 \mathrm{~g}$ (where $\mathrm{g}$ is the acceleration due to gravity) would be needed dislodge the fireproofing. Additional analytical studies will be conducted to account for dynamic effects, and tests will be performed to verify these predictions. 
. 
